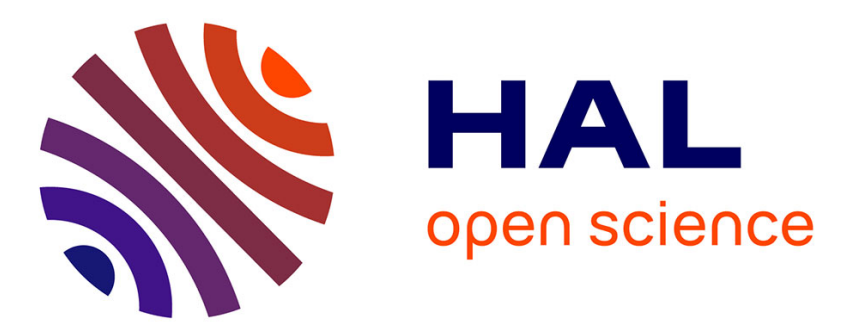

\title{
Asymptotic and non asymptotic approximations for option valuation
}

Romain Bompis, Emmanuel Gobet

\section{To cite this version:}

Romain Bompis, Emmanuel Gobet. Asymptotic and non asymptotic approximations for option valuation. Thomas Gerstner and Peter Kloeden. Computational finance, World scientific, pp.80, 2012. hal-00720650

\section{HAL Id: hal-00720650 https://hal.science/hal-00720650}

Submitted on 25 Jul 2012

HAL is a multi-disciplinary open access archive for the deposit and dissemination of scientific research documents, whether they are published or not. The documents may come from teaching and research institutions in France or abroad, or from public or private research centers.
L'archive ouverte pluridisciplinaire HAL, est destinée au dépôt et à la diffusion de documents scientifiques de niveau recherche, publiés ou non, émanant des établissements d'enseignement et de recherche français ou étrangers, des laboratoires publics ou privés. 


\title{
Chapter 1
}

\section{Asymptotic and non asymptotic approximations for option valuation}

\author{
R. Bompis and E. Gobet* \\ Centre de Mathématiques Appliquées, Ecole Polytechnique and CNRS, \\ Route de Saclay, 91128 Palaiseau cedex, France \\ romain.bompis@polytechnique.edu,emmanuel.gobet@polytechnique.edu
}

\begin{abstract}
We give a broad overview of approximation methods to derive analytical formulas for accurate and quick evaluation of option prices. We compare different approaches, from the theoretical point of view regarding the tools they require, and also from the numerical point of view regarding their performances. In the case of local volatility models with general time-dependency, we derive new formulas using the local volatility function at the mid-point between strike and spot: in general, our approximations outperform previous ones by Hagan and HenryLabordère. We also provide approximations of the option delta.
\end{abstract}

\section{Introduction}

In the two last decades, numerous works have been devoted to designing efficient methods in order to give exact or approximative pricing formulas for many financial products in various models. This quest of efficiency originates in the need for more and more accurate methods, when one takes into account an increasing number of sources of risk, while maintaining a competitive computational time. The current interest in real-time tools (for pricing, hedging, calibration) is also very high.

Let us give a brief overview of different computational approaches. While explicit formulas are available in simple models (Black-Scholes model associated to log-normal distribution, or Bachelier model related to normal distributions for instance), in general no closed forms are known and numerical methods have to be used. As a numerical method, it is usual to perform PDE solvers for one or twodimensional sources of risk (see [1] for instance) or Monte Carlo methods for higher dimensional problems [2]: both approaches are popular, efficiently developed and many improvements have been proposed for years. However, these methods are not intrinsically real-time methods, due to the increasing number of points required in the PDE discretization grid or due to the increasing number of paths needed in the Monte Carlo procedure. Not being real-time method means, for example, that when

* This research is part of the Chair Financial Risks of the Risk Foundation, the Chair Derivatives of the Future and the Chair Finance and Sustainable Development. 
used for calibration routine based on data consisting of (say) 30 vanilla options, it usually takes more than one minute (in the most favorable situation) to achieve the calibration parameters. The approaches presented below are aimed at reducing this computational time to less than one second.

The class of affine models (such as Heston model, exponential Levy model ... ) offers an alternative approach related to Fourier computations: on the one hand, in such models the characteristic function of the marginal distribution of the logasset is explicitly known; on the other hand, there are general relations between Call/Put prices and the characteristic function of the log-asset. These relations write as follows.

- Following Carr and Madan [3], consider the difference $z_{T}(k)$ between the Call price in a given model and that price in an arbitrary Black-Scholes model (with volatility $\sigma$ ), both with maturity $T$ and $\log$-strike $k$. For zero interest rate (to simplify), it is equal to $z_{T}(k)=\mathbb{E}\left(e^{X_{T}}-e^{k}\right)_{+}-\mathrm{Call}^{\mathrm{BS}}(k)$, where $X$ is the logarithm of the asset. A direct computation gives explicitly the Fourier transform $\widehat{z_{T}}(v)$ in the log-strike variable:

$$
\widehat{z_{T}}(v)=\int_{\mathbb{R}} e^{i v k} z_{T}(k) \mathrm{d} k=\frac{\Phi_{T}^{X}(1+i v)-\Phi_{T}^{\mathrm{BS}}(1+i v)}{i v(1+i v)},
$$

where $\Phi_{T}^{X, \mathrm{BS}}(u):=\mathbb{E}\left(e^{u X_{T}}\right)$ is either computed in the $X$ model or in the Black-Scholes model. Since $\Phi_{T}^{X}(\cdot)$ is required to be known, we get the $X$ model Call price $z_{T}(k)+$ Call $^{\mathrm{BS}}(k)$ simultaneously for any log-strike using a Fast Fourier Transform.

- Alternatively, following the Lewis approach [4, Chapter 2], let $\alpha>0$ be a damping constant, set $h(y)=\left(e^{y}-K\right)_{+} e^{-(1+\alpha) y}$ which belongs to $\mathbf{L}_{2}(\mathbb{R}$, Leb. $)$ and assume that $\mathbb{E}\left(e^{(1+\alpha) X_{T}}\right)<+\infty$ : from the ParcevalPlancherel identity, assuming that the density $p_{X_{T}}$ of $X_{T}$ w.r.t. the Lebesgue measure exists, we obtain

$$
\begin{aligned}
\mathbb{E}\left(e^{X_{T}}-K\right)_{+} & =\int_{\mathbb{R}} h(y) e^{(1+\alpha) y} p_{X_{T}}(y) \mathrm{d} y \\
& =\frac{1}{2 \pi} \int_{\mathbb{R}} \widehat{h}(-\xi)\left[e^{(1+\alpha) \cdot p_{X_{T}}}(\cdot)\right](\xi) \mathrm{d} \xi \\
& =\frac{1}{2 \pi} \int_{\mathbb{R}} \frac{e^{-(\alpha+i \xi) \log (K)}}{(i \xi+\alpha)(i \xi+\alpha+1)} \Phi_{T}^{X}(1+\alpha+i \xi) \mathrm{d} \xi
\end{aligned}
$$

The final identity still holds without assuming the existence of density: this can be proved by adding a small Brownian perturbation (considering $X_{T}+\varepsilon W_{T}$ instead of $X_{T}$ ), and taking the limit as the perturbation $\varepsilon$ goes to 0 . From the above formula, using an extra numerical integration method (to compute the $\xi$-integral), we recover Call prices. For higher numerical performance, Lewis recommends a variant of the formula above, obtained through the decomposition $\left(e^{X_{T}}-K\right)_{+}=e^{X_{T}}-\min \left(e^{X_{T}}, K\right)$ : it finally 
writes

$$
\mathbb{E}\left(e^{X_{T}}-K\right)_{+}=S_{0}-\frac{1}{2 \pi} \int_{\mathbb{R}} \frac{e^{\left(\frac{1}{2}-i \xi\right) \log (K)}}{\frac{1}{4}+\xi^{2}} \Phi_{T}^{X}\left(\frac{1}{2}+i \xi\right) \mathrm{d} \xi .
$$

Regarding computational time, both Fourier-based approaches perform well, since they are essentially reduced to a one-dimensional integration problem. But they can be applied only to specific models for which the characteristic function is given in an explicit and tractable form: in particular, it rules out the local volatility models, the local and stochastic volatility models.

The last approach consists of explicit analytical approximations and this is the main focus of this paper: it is based on the general principle of expanding the quantity of interest (price, hedge, implied volatility... ) with respect to some small/large parameters (possibly multidimensional). The parameters under consideration may be of very different nature: for instance in the case of Call/Put options of strike $K$ and maturity $T$, it ranges from the asymptotic behavior as $K$ is small or large, to the case of short or long maturity $T$, passing through coupled asymptotics, or small/fast volatility variations, and so on... A detailed description with references is presented in Section 2. Due to the plentiful and recent litterature on the subject, it is likely that we will not be exhaustive in the references. But we will do our best to give the main trends and to expose whenever possible what are the links between different viewpoints; we will compare the mathematical tools to achieve these approximations (rather PDE techniques or stochastic analysis ones), in order to provide to the reader a clarified presentation of this prolific topic.

The paper is organized as follows. Section 2 gives an overview of asymptotic and non-asymptotic results: wing formulas, long maturity behavior, large deviations type results, regular and singular perturbation for PDEs, asymptotic expansions of Wiener functionals and other stochastic analysis approaches. The choice of the small/large parameter is of course crucial and is usually left to the expertise of the user. In particular, we show that there might be a competition between different small/large parameters and the accuracy order might not be the natural one. This motivates for deriving non asymptotic results and this is our emphasize in the next sections. We develop the principle of high order approximations related to an intuitive proxy. In Section 3, we consider the simplest case of second order approximation in local volatility models, using log-normal or normal proxys. We give pedagogic proofs. Section 4 is devoted to a more detailed analysis: we first give arguments based on stochastic analysis (martingales, Malliavin calculus). We compare this derivation with a method mixing stochastic analysis and PDE, and with a pure PDE approach: we show in which respect our methodology is different. In Section 5, we provide various high-order approximation using proxys. In Section 6, approximations of the option delta are provided. Section 7 is gathering numerical experiments illustrating the performance of our formulas compared to those of Hagan etal. [5] and of Henry-Labordère [6]. Some intermediate and complementary results are postponed to Appendix (Section 8). 


\section{Notation used throughout the paper.}

$\triangleright$ Models. In all this work, financial products are written w.r.t. a single asset, which price at time $t$ is denoted by $S_{t}$. The dynamics of $S$ is modeled through a filtered probability space $\left(\Omega, \mathcal{F},\left(\mathcal{F}_{t}\right)_{t>0}, \mathbb{P}\right)$ where $\left(\mathcal{F}_{t}\right)_{t>0}$ is the natural filtration of a standard linear Brownian motion $W$, augmented by the $\mathbb{P}$-null sets. The risk-free rate is set ${ }^{\mathrm{a}}$ to 0 ; most of the time and unless stated otherwise, $S$ follows a local volatility model, i.e. it is solution of the stochastic diffusion equation

$$
\mathrm{d} S_{t}=S_{t} \sigma\left(t, S_{t}\right) \mathrm{d} W_{t},
$$

where the dynamics is directly under the pricing measure. Assumptions on the local volatility $\sigma$ are given later. We assume that the complete market framework holds and that an option with payoff $h\left(S_{T}\right)$ paid at maturity $T$ has a fair value at time 0 equal to $\mathbb{E}\left(h\left(S_{T}\right)\right)$.

For positive $S$, we define the $\log$-asset $X=\log (S)$ which satisfies

$$
\mathrm{d} X_{t}=a\left(t, X_{t}\right) \mathrm{d} W_{t}-\frac{1}{2} a^{2}\left(t, X_{t}\right) \mathrm{d} t,
$$

where $a(t, x)=\sigma\left(t, e^{x}\right)$.

$\triangleright$ Call options. Let us denote by $\operatorname{Call}\left(S_{0}, T, K\right)$ the price at time 0 of a Call option with maturity $T$ and strike $K$, written on the asset $S$. "Price" usually means the price given by a model on $S$, that is

$$
\operatorname{Call}\left(S_{0}, T, K\right)=\mathbb{E}\left(S_{T}-K\right)_{+} .
$$

This Model Price should equalize the Market Price taken from Market datas (calibration step). As usual, ATM (At The Money) Call refers to $S_{0} \approx K$, ITM (In The Money) to $S_{0} \gg K$, OTM (Out The Money) to $S_{0} \ll K$.

$\triangleright$ Black-Scholes Call price function. For convenience of the reader, we give the Black-Scholes Call price function depending on log-spot $x$, total variance $y$ and $\log$-strike $z$ :

$$
\operatorname{Call}^{\mathrm{BS}}(x, y, z)=e^{x} \mathcal{N}\left(d_{1}(x, y, z)\right)-e^{k} \mathcal{N}\left(d_{2}(x, y, z)\right)
$$

where:

$$
\begin{aligned}
\mathcal{N}(x) & =\int_{-\infty}^{x} \mathcal{N}^{\prime}(u) \mathrm{d} u, & \mathcal{N}^{\prime}(u) & =\frac{e^{-u^{2} / 2}}{\sqrt{2 \pi}}, \\
d_{1}(x, y, z) & =\frac{x-z}{\sqrt{y}}+\frac{1}{2} \sqrt{y}, & d_{2}(x, y, z) & =d_{1}(x, y, z)-\sqrt{y} .
\end{aligned}
$$

This value Call ${ }^{\mathrm{BS}}(x, y, z)$ equals Call $\left(e^{x}, T, e^{z}\right)$ in (4) when the volatility in (2) is only time-dependent and $y=\int_{0}^{T} \sigma^{2}(t) \mathrm{d} t$. Note that Call ${ }^{\mathrm{BS}}$ is a smooth function (for $y>0$ ) and there is in addition a simple relation between its partial derivatives:

$$
\partial_{y} \mathrm{Call}^{\mathrm{BS}}(x, y, z)=\frac{1}{2}\left(\partial_{x^{2}}^{2}-\partial_{x}\right) \mathrm{Call}^{\mathrm{BS}}(x, y, z)=\frac{1}{2}\left(\partial_{z^{2}}^{2}-\partial_{z}\right) \mathrm{Call}^{\mathrm{BS}}(x, y, z) .
$$

${ }^{a}$ for non-zero but deterministic risk-free rate, we are reduced to the previous case by considering the discounted asset; see also the discussion in [7] for stochastic interest rates. 
In the following, $x_{0}=\log \left(S_{0}\right)$ (which is the initial value of the process $X$ defined in (3)) will represent the $\log$-spot, $k=\log (K)$ the log-strike, $x_{\mathrm{av}}=\left(x_{0}+k\right) / 2=$ $\log \left(\sqrt{S_{0} K}\right)$ the mid-point between the log-spot and the log-strike, $m=x_{0}-k=$ $\log \left(S_{0} / K\right)$ the log-moneyness.

The reader can find in Proposition 32 the definition of $\mathrm{Vega}^{\mathrm{BS}}$, Vomma ${ }^{\mathrm{BS}}$ and Ultima $^{\mathrm{BS}}$ which are the first three derivatives of Call ${ }^{\mathrm{BS}}$ w.r.t. a volatility parameter.

For $(x, T, z)$ given, the implied Black-Scholes volatility of a price Call $\left(e^{x}, T, e^{z}\right)$ is the unique non-negative parameter $\sigma_{\mathrm{I}}(x, T, z)$ such that

$$
\operatorname{Call}^{\mathrm{BS}}\left(x, \sigma_{\mathrm{I}}^{2}(x, T, z) T, z\right)=\operatorname{Call}\left(e^{x}, T, e^{z}\right) .
$$

$\triangleright$ Bachelier Call price function. We now recall the Bachelier Call price as a function of spot $S$, total variance $Y$ and strike $Z$ :

$$
\mathrm{Call}^{\mathrm{BA}}(S, Y, Z)=(S-Z) \mathcal{N}\left(\frac{S-Z}{\sqrt{Y}}\right)+\sqrt{Y} \mathcal{N}^{\prime}\left(\frac{S-Z}{\sqrt{Y}}\right),
$$

which coincides with $\operatorname{Call}(S, T, Z)$ when the volatility in (2) is such that $x \sigma(t, x)=$ $\Sigma(t)$ and $Y=\int_{0}^{T} \Sigma^{2}(t) \mathrm{d} t$. The function Call ${ }^{\mathrm{BA}}$ is smooth (for $Y>0$ ) and we have:

$$
\partial_{Y} \operatorname{Call}^{\mathrm{BA}}(S, Y, Z)=\frac{1}{2} \partial_{S^{2}}^{2} \operatorname{Call}^{\mathrm{BA}}(S, Y, Z)=\frac{1}{2} \partial_{Z^{2}}^{2} \mathrm{Call}^{\mathrm{BA}}(S, Y, Z) .
$$

We frequently use the notation $S_{\mathrm{av}}=\left(S_{0}+K\right) / 2$ and $M=S_{0}-K$ for the Bachelier moneyness. Proposition 37 defines the sensibilities of $\mathrm{Call}^{\mathrm{BA}}$ w.r.t. the volatility parameter: Vega ${ }^{\mathrm{BA}}$, Vomma ${ }^{\mathrm{BA}}$ and Ultima ${ }^{\mathrm{BA}}$.

For $(S, T, Z)$ given, the implied Bachelier volatility of a price $\operatorname{Call}(S, T, Z)$ is the unique non-negative parameter $\Sigma_{\mathrm{I}}(S, T, Z)$ such that

$$
\mathrm{Call}^{\mathrm{BA}}\left(S, \Sigma_{\mathrm{I}}^{2}(S, T, Z) T, Z\right)=\operatorname{Call}(S, T, Z) .
$$

Black-Scholes and Bachelier implied volatilities are compared in [8].

\section{An overview of approximation results}

The increasing need in evaluating financial risks at a very global level and in a context of high-frequency market exchanges is a significant incentive for the computational methods to be efficient in evaluating the exposure of large portfolio to market fluctuations (VaR computations, sensitivity analysis), in quickly calibrating the models to the market data. Hence, in the two last decades, many numerical methods have been developed to meet these objectives: in particular, regarding the option pricing, several approximation results have been derived, following one or another asymptotic point of view. We give a summary of these different approaches, stressing the limits of applicability of the methods. 


\subsection{Large and small strikes, at fixed maturity}

The Call price Call $\left(S_{0}, T, K\right)$ as a function of strike is convex and its left/right derivatives are related to the distribution function of $S_{T}$ [9, Chapter 7]: $\partial_{K}^{-} \operatorname{Call}\left(S_{0}, T, K\right)=-\mathbb{P}\left(S_{T} \geq K\right)$ and $\partial_{K}^{+} \operatorname{Call}\left(S_{0}, T, K\right)=-\mathbb{P}\left(S_{T}>K\right)$. Beyond the important fact that the family of Call prices $\left\{\operatorname{Call}\left(S_{0}, T, K\right): K \geq 0\right\}$ completely characterizes the marginal distribution of $S_{T}$, this relation also shows that the tails of the law of $S_{T}$ are intrinsically related to the decay of $\operatorname{Call}\left(S_{0}, T, K\right)$ as $K \rightarrow+\infty$. In terms of implicit volatility, the heuristics is the following: the larger the implied volatility of OTM options, the larger the right tail of $S_{T}$. This is similar for small strikes $K$, using Put options. Lee [10] has been the first one to quantify these features relating the behavior of implied volatility to the tails of $S_{T}$, with an encoding of the tails through the existence of positive/negative moments. These are model-free relations, that can be applied to any model with $\mathbb{E}\left(S_{T}\right)<+\infty$ and not only to local volatility ones like in (2). The well-known Lee moment formulas write as follows, using the $\log$-variables $x_{0}=\log \left(S_{0}\right)$ and $m=\log \left(S_{0} / K\right)=x_{0}-k$.

Theorem 1. Define

- the maximal finite positive moment order $p_{R}:=\sup \left\{p \geq 0: \mathbb{E}\left(S_{T}^{1+p}\right)<+\infty\right\}$,

- the maximal finite negative moment order ${ }^{\mathrm{b}} p_{L}:=\sup \left\{p \geq 0: \mathbb{E}\left(S_{T}^{-p}\right)<+\infty\right\}$. Then, the right tail-wing of the Black-Scholes implied volatility defined in (7) is such that

$$
\limsup _{m \rightarrow-\infty} \frac{T \sigma_{\mathrm{I}}^{2}\left(x_{0}, T, x_{0}-m\right)}{|m|}=\phi\left(p_{R}\right):=\beta_{R},
$$

while the left tail-wing is such that

$$
\limsup _{m \rightarrow+\infty} \frac{T \sigma_{\mathrm{I}}^{2}\left(x_{0}, T, x_{0}-m\right)}{m}=\phi\left(p_{L}\right):=\beta_{L},
$$

where $\phi(x)=2-4\left(\sqrt{x^{2}+x}-x\right) \in[0,2]$.

Proof. We refer to [10] for a detailed proof. We only give the two main arguments for proving the right tail-wing, the left one being similar.

- The first argument relies on a tight connection between moments and asymptotics of Call/Put as $K \rightarrow+\infty$. Indeed, on the one hand, convexity inequalities give $(s-K)_{+} \leq \frac{s^{p+1}}{p+1}\left(\frac{p}{p+1}\right)^{p} \frac{1}{K^{p}}$ (for $\left.p \geq 0\right)$, and taking the expectation yields

$$
\operatorname{Call}\left(S_{0}, T, K\right) \leq \frac{\mathbb{E}\left(S_{T}^{p+1}\right)}{p+1}\left(\frac{p}{p+1}\right)^{p} \frac{1}{K^{p}} .
$$

In other words, the more integrability of $S_{T}$, the faster the decay of Call $\left(S_{0}, T, K\right)$ as $K \rightarrow+\infty$. Conversely, the Carr formula states that the Call/Put prices form a

$\overline{\mathrm{b}} 1+p_{R}$ and $p_{L}$ are respectively called right-tail and left-tail indices. 
pricing generating system for any payoff equal to a difference of convex functions: making this principle particular to the power payoff, we obtain

$$
\mathbb{E}\left(S_{T}^{1+p}\right)=\int_{0}^{\infty} p(p+1) K^{p-1} \operatorname{Call}\left(S_{0}, T, K\right) \mathrm{d} K,
$$

i.e. the faster the decreasing of $\operatorname{Call}\left(S_{0}, T, K\right)$ as $K \rightarrow+\infty$, the higher the integrability of $S_{T}$.

- The second argument is based on exponential decreasing behaviors of Call/Put in terms of Black-Scholes implied volatility, as the log-moneyness $m \rightarrow \pm \infty$. Reparameterizing the implied volatility $\sigma_{\mathrm{I}}\left(x_{0}, T, x_{0}-m\right)=\sqrt{\beta|m| / T}$ with $\beta \in(0,2](\beta$ is interpreted as a slope of the total variance per log-moneyness), we obtain

$$
\mathrm{Call}^{\mathrm{BS}}\left(x_{0}, \beta|m|, x_{0}-m\right)=S_{0} \mathcal{N}\left(-\sqrt{f_{-}(\beta)|m|}\right)-S_{0} e^{-m} \mathcal{N}\left(-\sqrt{f_{+}(\beta)|m|}\right)
$$

where $f_{ \pm}(\beta)=\frac{1}{\beta}+\frac{\beta}{4} \pm 1$. Then, a direct computation shows a dichotomic behavior related to $\beta$ :

$$
\lim _{m \rightarrow-\infty} e^{-c m} \mathrm{Call}^{\mathrm{BS}}\left(x_{0}, \beta|m|, x_{0}-m\right)=+\infty \mathbf{1}_{c>f_{-}(\beta) / 2} .
$$

Comparing (10-11-12) and setting $p_{R}:=f_{-}\left(\beta_{R}\right) / 2$ (or equivalently $\beta_{R}=\phi\left(p_{R}\right)$ ) yields the tail-wing formulas.

Since the original contribution of Lee, several improvements to Theorem 1 have been established. For instance, the lim sup can be removed by a simple limit, under the additional assumptions that $S_{T}$ has a regularly varying density, see [11]. More recently, Gulisashvili [12] and his co-authors have proved refined expansions of the form

$$
\begin{gathered}
\sigma_{\mathrm{I}}\left(x_{0}, T, k\right)=\frac{\sqrt{2}}{\sqrt{T}}\left[\sqrt{\log K+\log \frac{1}{\operatorname{Call}\left(S_{0}, T, K\right)}-\frac{1}{2} \log \log \frac{1}{\operatorname{Call}\left(S_{0}, T, K\right)}}\right. \\
\left.-\sqrt{\log \frac{1}{\operatorname{Call}\left(S_{0}, T, K\right)}-\frac{1}{2} \log \log \frac{1}{\operatorname{Call}\left(S_{0}, T, K\right)}}\right]+O\left(\left(\log \frac{1}{\operatorname{Call}\left(S_{0}, T, K\right)}\right)^{-\frac{1}{2}}\right)
\end{gathered}
$$

as $K$ becomes large, which allows precise asymptotics of $\sigma_{\mathrm{I}}\left(x_{0}, T, k\right)$ through those of $\operatorname{Call}\left(S_{0}, T, K\right)$.

These kinds of asymptotics are now well-known for most of the usual models, like CEV models (no right tail-wing), Heston model (tail-wing depending on the maturity)... see [13] for more references. Different models may have the same strike asymptotics. We can use this information on extreme strikes in different manners: first, comparing with the asymptotic market implied volatility smile, it allows for selecting a coherent model. Second, it helps the calibration procedure by setting approximately some parameter values (those having an impact on the tails). Third, it can be used to appropriately extrapolate market data.

In practice, these asymptotic formulas refer to far OTM or ITM options, for which the accuracy of market data is really questionable (large bid-ask spread, low liquidity). Thus, a direct application is usually not straightforward. 


\subsection{Long maturities, at fixed strike}

Another asymptotics is large maturity. It has been studied by Rogers and Tehranchi, see [14] and [15], proving the following.

Theorem 2. Assume that $S$ remains positive with probability 1 . Then, for any $\lambda>0, \lim _{T \rightarrow+\infty} \sup _{|m| \leq \lambda}\left|\sigma_{\mathrm{I}}\left(x_{0}, T, x_{0}-m\right)-\sqrt{\frac{8}{T}\left|\ln \left(\mathbb{E}\left(S_{T} \wedge S_{0}\right)\right)\right|}\right|=0$.

As before, the proof is based on the careful derivation of asymptotics of BlackScholes formula (5). The above limit states that for strikes in a fixed neighborhood of the spot $S_{0}$, the implied volatility behaves like $\sqrt{\frac{8}{T}\left|\ln \left(\mathbb{E}\left(S_{T} \wedge S_{0}\right)\right)\right|}$ for large maturity, and thus it does not depend on the strike. In other words, the implied volatility surface flattens as maturity becomes large, which is coherent with market data. There are also some refined and higher order asymptotics: assuming that the a.s. large-time limit of the martingale $S$ is 0 , then

$$
T \sigma_{\mathrm{I}}^{2}\left(x_{0}, T, k\right)=8\left|\ln \left(\mathbb{E}\left(S_{T} \wedge \frac{K}{S_{0}}\right)\right)\right|-4 \ln \left(\left|\ln \left(\mathbb{E}\left(S_{T} \wedge \frac{K}{S_{0}}\right)\right)\right|\right)+4 \ln \left(\frac{K}{\pi S_{0}}\right)+o(1),
$$

where the reminder is locally uniform in the log-moneneyss $m=x_{0}-k$.

\subsection{Long maturities, with large/small strikes}

In view of the preceeding results, the asymptotics of the smile for large maturity becomes very simple regarding the strike variable, unless one allows the strike to be large/small together with the maturity. Indeed to recover interesting information at the limit, we should consider strikes of the form $K=S_{0} e^{x T}$ with $x \neq 0$, or equivalently $k=x_{0}+x T$. From the linearization of the payoff, one obtains

$$
\begin{aligned}
\operatorname{Call}\left(S_{0}, T, S_{0} e^{x T}\right) & =\mathbb{E}\left(S_{T} \mathbf{1}_{S_{T} \geq S_{0} e^{x T}}\right)-S_{0} e^{x T} \mathbb{P}\left(S_{T} \geq S_{0} e^{x T}\right) \\
& =S_{0} \mathbb{P}^{S}\left(\frac{1}{T} \log \left(S_{T} / S_{0}\right) \geq x\right)-S_{0} e^{x T} \mathbb{P}\left(\frac{1}{T} \log \left(S_{T} / S_{0}\right) \geq x\right)
\end{aligned}
$$

where the new measure $\mathbb{P}^{S}$ is the one associated to the numéraire $S$. Under this form, it appears clearly that for $x$ large enough (say larger than the asymptotic $\mathbb{P}$-mean or $\mathbb{P}^{S}$-mean of $\frac{1}{T} \log \left(S_{T} / S_{0}\right)$ whenever it exists $)$, both probabilities above correspond to the evaluation of large deviation events. The role of Large Deviation Principle satisfied by the sequence $\left(\frac{1}{T} \log \left(S_{T} / S_{0}\right)\right)_{T \geq 0}$ as $T \rightarrow+\infty$ has been outlined in [16] in the case of Heston model, and in [17] for more general affine models. Saddle point arguments combined with Lewis formula (1) have been performed in [18] for the Heston model, to recover the Stochastic Volatility Inspired parameterization of Gatheral [19]: the squared implied volatility $\sigma_{\mathrm{I}}^{2}\left(x_{0}, T, x_{0}+x T\right)$ has the simple asymptotic shape

$$
\sigma_{\infty}^{2}(x)=\frac{\omega_{1}}{2}\left(1+\omega_{2} \rho x+\sqrt{\left(\omega_{2} x+\rho\right)^{2}+1-\rho^{2}}\right) .
$$

For more general affine models like Heston model, without or with jumps, or Bates model, or Barndorff-Nielsen-Shephard model (see [20] and [17]), it is possible to 
derive similar limits. Let $\Lambda_{t}(u)=\log \left(\mathbb{E}\left(S_{t}^{u}\right)\right)$ be the exponent of the moment generating function, which is convex in $u$ : in the aforementioned model we can define and compute its asymptotic average $\Lambda(u)=\lim _{t \rightarrow \infty} \frac{1}{t} \Lambda_{t}(u)$, which still satisfies to the convexity feature. We associate its Fenchel-Legendre transform $\Lambda^{*}(x)=$ $\sup _{u \in \mathbb{R}}(u x-\Lambda(u))$ and it turns out that $\left(\frac{1}{T} \log \left(S_{T} / S_{0}\right)\right)_{T \geq 0}$ satisfies a LDP under $\mathbb{P}\left(\right.$ resp. $\mathbb{P}^{S}$ ) with rate function $x \mapsto \Lambda^{*}(x)$ (resp. $x \mapsto \Lambda^{*}(x)-x$ ).

Theorem 3. Under some assumptions (see [17]), for any $x \in \mathbb{R}$, the asymptotic implied volatility $\sigma_{\infty}(x)$ is given by

$\lim _{T \rightarrow \infty} \sigma_{\mathrm{I}}\left(x_{0}, T, x_{0}+x T\right)=\sqrt{2}\left[\operatorname{sgn}\left(\Lambda^{\prime}(1)-x\right) \sqrt{\Lambda^{*}(x)-x}+\operatorname{sgn}\left(x-\Lambda^{\prime}(0)\right) \sqrt{\Lambda^{*}(x)}\right]$.

In the Black-Scholes model with constant volatility $\sigma$, one has $\Lambda(u)=\frac{\sigma^{2}}{2}\left(u^{2}-u\right)$, $\Lambda^{*}(x)=\frac{1}{2 \sigma^{2}}\left(x+\frac{\sigma^{2}}{2}\right)^{2}$, and we get obviously $\sigma_{\infty}(x)=\sigma$. For Heston model, $\Lambda$ is explicit as well and we finally recover the SVI parsimonious parameterization (13). Here again, different models may have the same asymptotic smiles, see [17].

\subsection{Non large maturities and non extreme strikes}

To obtain approximation formulas in that situation, the asymptotics should originate from different large/small parameters that are rather related to the model and not to the contract characteristics (maturity and strike). These different asymptotics are generally well intuitively interpreted. For the sake of clarity, we spend time to detail a bit the arguments, in order to make clearer the differences between the further expansion results and the tools to obtain them. To the best of our knowledge, such comparative presentation does not exist in the literature and the reader may find it interesting.

\subsubsection{Small noise expansion}

This is inspired by the Freidlin-Wentzell approach [21] in which the noise in the Stochastic Differential Equation of interest is small. Denote by $Y$ the scalar SDE under study (which can be $X$ or $S$ in our framework), solution of

$$
\mathrm{d} Y_{t}=\mu\left(t, Y_{t}\right) \mathrm{d} t+\nu\left(t, Y_{t}\right) \mathrm{d} W_{t}, \quad Y_{0} \text { given } .
$$

Assume that $\nu$ is small, or equivalently that $\nu$ becomes $\varepsilon \nu$ with $\varepsilon \rightarrow 0$ : after making this small noise parameterization, the model writes

$$
\mathrm{d} Y_{t}^{\varepsilon}=\mu\left(t, Y_{t}^{\varepsilon}\right) \mathrm{d} t+\varepsilon \nu\left(t, Y_{t}^{\varepsilon}\right) \mathrm{d} W_{t}, \quad Y_{0}^{\epsilon}=Y_{0} .
$$

For $\varepsilon=0$, it reduces to an ODE

$$
Y_{t}^{0}=y_{0, t}=Y_{0}+\int_{0}^{t} \mu\left(s, y_{0, s}\right) \mathrm{d} s
$$


and this deterministic model serves as a zero-order approximation for the further expansion. Under smooth coefficient assumptions [21], we can derive a stochastic expansion of $Y^{\varepsilon}$ in powers of $\varepsilon$ :

$$
Y_{t}^{\varepsilon}=y_{0, t}+\varepsilon Y_{1, t}+\frac{1}{2} \varepsilon^{2} Y_{2, t}+o\left(\varepsilon^{2}\right) .
$$

For instance $Y_{1}$ solves a linear Gaussian SDE

$$
Y_{1, t}=\int_{0}^{t} \partial_{x} \mu\left(s, y_{0, s}\right) Y_{1, s} \mathrm{~d} s+\int_{0}^{t} \nu\left(s, y_{0, s}\right) \mathrm{d} W_{s}=\int_{0}^{t} e^{\int_{s}^{t} \partial_{x} \mu\left(r, y_{0, r}\right) \mathrm{d} r} \nu\left(s, y_{0, s}\right) \mathrm{d} W_{s} .
$$

Similarly, $Y_{2}$ solves

$$
\begin{aligned}
Y_{2, t} & =\int_{0}^{t}\left[\partial_{x} \mu\left(s, y_{0, s}\right) Y_{2, s}+\partial_{x}^{2} \mu\left(s, y_{0, s}\right) Y_{1, s}^{2}\right] \mathrm{d} s+\int_{0}^{t} 2 \partial_{x} \nu\left(s, y_{0, s}\right) Y_{1, s} \mathrm{~d} W_{s} \\
& =\int_{0}^{t} e^{\int_{s}^{t} \partial_{x} \mu\left(r, y_{0, r}\right) d r}\left(\partial_{x}^{2} \mu\left(s, y_{0, s}\right) Y_{1, s}^{2} \mathrm{~d} s+2 \partial_{x} \nu\left(s, y_{0, s}\right) Y_{1, s} \mathrm{~d} W_{s}\right) .
\end{aligned}
$$

Higher order expansions are available under higher smoothness assumptions. The notation $o\left(\varepsilon^{2}\right)$ in (16) means that the related error term has a $L_{p}$-norm (for any $p$ ) that can be neglected compared to $\varepsilon^{2}$ as $\varepsilon \rightarrow 0$. The stochastic expansion (16) becomes a weak expansion result when we compute $\mathbb{E}\left(h\left(Y_{T}\right)\right)$ for a test function $h$.

$\triangleright$ THE CASE OF SMOOTH $h$. If $h$ is smooth enough, we obviously obtain

$$
\begin{aligned}
\mathbb{E}\left(h\left(Y_{T}\right)\right)= & h\left(y_{0, T}\right)+\varepsilon h^{\prime}\left(y_{0, T}\right) \mathbb{E}\left(Y_{1, T}\right) \\
& +\varepsilon^{2}\left(h^{\prime}\left(y_{0, T}\right) \mathbb{E}\left(\frac{Y_{2, T}}{2}\right)+\frac{1}{2} h^{\prime \prime}\left(y_{0, T}\right) \mathbb{E}\left(Y_{1, T}^{2}\right)\right)+o\left(\varepsilon^{2}\right) .
\end{aligned}
$$

Observe that $\mathbb{E}\left(Y_{1, T}\right)=0$ since $Y_{1, T}$ is a Wiener integral. To make the above expansion fully effective in practice, it is necessary to make the coefficients $\mathbb{E}\left(Y_{2, T}\right)$ and $\mathbb{E}\left(Y_{1, T}^{2}\right)$ explicit: this is quite straightforward thanks to the linear equations solved by $Y_{1, .}$ and $Y_{2, .}$. The $L_{2}$-isometry property of the Wiener integral yields $\mathbb{E}\left(Y_{1, t}^{2}\right)=\int_{0}^{t} e^{2 \int_{s}^{t} \partial_{x} \mu\left(r, y_{0}, r\right) \mathrm{d} r} \nu^{2}\left(s, y_{0, s}\right) \mathrm{d} s$. In addition, we have $\mathbb{E}\left(Y_{2, t}\right)=$ $\int_{0}^{t} e^{\int_{s}^{t} \partial_{x} \mu\left(r, y_{0, r}\right) \mathrm{d} r} \partial_{x}^{2} \mu\left(s, y_{0, s}\right) \mathbb{E}\left(Y_{1, s}^{2}\right) \mathrm{d} s$. The coefficients computation is reduced to the evaluation of nested time-integrals which are simple to compute using standard $n$-points integral discretization, with a computational complexity ${ }^{\mathrm{c}}$ of order $n$. The above expansion analysis is a regular perturbation analysis, using a stochastic analysis point of view.

To derive this expansion in powers of $\varepsilon$, we could alternatively use a PDE point of view based on Feynman-Kac representation, which states that $u^{\varepsilon}:(t, x) \mapsto$ $u^{\varepsilon}(t, x)=\mathbb{E}\left(h\left(Y_{T}^{\varepsilon}\right) \mid Y_{t}^{\varepsilon}=x\right)$ solves the perturbed PDE

$$
\left\{\begin{array}{l}
\partial_{t} u^{\varepsilon}(t, x)+\mu(t, x) \partial_{x} u^{\varepsilon}(t, x)+\frac{1}{2} \varepsilon^{2} \nu^{2}(t, x) \partial_{x}^{2} u^{\varepsilon}(t, x)=0 \quad \text { for } t<T, \\
u^{\varepsilon}(T, x)=h(x) .
\end{array}\right.
$$

${ }^{\mathrm{c}}$ Observe that although the time integrals are multidimensional, we are reduced to iterative onedimensional computations since the function to integrate is separable in all its variables. 
Setting $\mathcal{L}^{\varepsilon}=\partial_{t}+\mu \partial_{x}+\frac{1}{2} \varepsilon^{2} \nu^{2} \partial_{x}^{2}:=\mathcal{L}_{0}+\varepsilon^{2} \mathcal{L}_{2}$, the above PDE writes $\mathcal{L}^{\varepsilon} u^{\varepsilon}=0$ plus boundary conditions at time $T$. Seeking an expansion of the form $u^{\varepsilon}=u_{0}+$ $\varepsilon u_{1}+\frac{1}{2} \varepsilon^{2} u_{2}+o\left(\varepsilon^{2}\right)$, we obtain

$$
\mathcal{L}_{0} u_{0}+\varepsilon \mathcal{L}_{0} u_{1}+\varepsilon^{2}\left[\frac{1}{2} \mathcal{L}_{0} u_{2}+\mathcal{L}_{2} u_{0}\right]+o\left(\varepsilon^{2}\right)=0 .
$$

A formal identification of each coefficient of $\varepsilon^{i}(i=0,1 \ldots)$ to 0 , we obtain a system of PDEs:

$$
\mathcal{L}_{0} u_{0}=0, \quad \mathcal{L}_{0} u_{1}=0, \quad \frac{1}{2} \mathcal{L}_{0} u_{2}+\mathcal{L}_{2} u_{0}=0,
$$

with the boundary conditions $u_{0}(T,)=.h(),. u_{1}(T,)=.u_{2}(T,)=$.0 . The justification of this kind of expansion and its related error analysis can be made under appropriate smoothness assumptions on $h, \mu$ and $\nu$; we refer to [22, Theorem 5.1], [23, Theorem 3.1] or [24, Chapter 4] where a similar error analysis is made. The PDE solutions are then given by

$$
u_{0}(t, x)=h\left(y_{T}^{t, x}\right), \quad u_{1} \equiv 0, \quad u_{2}(t, x)=\int_{t}^{T} 2 \mathcal{L}_{2} u_{0}\left(s, y_{s}^{t, x}\right) \mathrm{d} s
$$

where $\left(y_{s}^{t, x}\right)_{s \geq t}$ stands for the solution of the ODE (15) with initial condition $(t, x)$. Under this form of system of PDEs, the derivation of an explicit expression for $u_{2}$ is not as easy as within the stochastic analysis approach. However, we can obtain the same expansion (fortunately!), i.e. the same formula for $u_{2}$ at $\left(0, Y_{0}\right)$ :

$$
u_{2}\left(0, Y_{0}\right)=h^{\prime}\left(y_{0, T}\right) \mathbb{E}\left(Y_{2, T}\right)+h^{\prime \prime}\left(y_{0, T}\right) \mathbb{E}\left(Y_{1, T}^{2}\right)
$$

with $\mathbb{E}\left(Y_{2, T}\right)$ and $\mathbb{E}\left(Y_{1, T}^{2}\right)$ given as before. To see this, start from $\mathcal{L}_{2}$ and write $u_{2}(t, x)=\int_{t}^{T} \nu^{2}\left(s, y_{s}^{t, x}\right) \partial_{y}^{2} u_{0}\left(s, y_{s}^{t, x}\right) \mathrm{d} s$. We have $\partial_{x} u_{0}(t, x)=h^{\prime}\left(t, y_{T}^{t, x}\right) \partial_{x} y_{T}^{t, x}$ and $\partial_{x}^{2} u_{0}(t, x)=h^{\prime}\left(t, y_{T}^{t, x}\right) \partial_{x}^{2} y_{T}^{t, x}+h^{\prime \prime}\left(t, y_{T}^{t, x}\right)\left(\partial_{x} y_{T}^{t, x}\right)^{2}$. Then to recover (17), use the notation $y_{0, t}=y_{t}^{0, Y_{0}}$, the flow property $y_{s}^{t, y_{0}, t}=y_{0, s}$ for $s \geq t$, and the explicit expressions for $\partial_{x} y_{s}^{t, x}$ and $\partial_{x}^{2} y_{s}^{t, x}$ : for instance $\partial_{x} y_{s}^{t, x}=1+\int_{t}^{s} \partial_{x} \mu\left(r, y_{r}^{t, x}\right) \partial_{x} y_{r}^{t, x} \mathrm{~d} r=$ $e^{\int_{t}^{s} \partial_{x} \mu\left(r, y_{r}^{t, x}\right) \mathrm{d} r}$. We skip further details. This completes the $P D E$ approach to derive a regular perturbation analysis. Observe that the derivation of explicit formula is delicate because of the system of PDEs to solve (more complicate than solving the system of SDEs arising within the stochastic analysis approach).

$\triangleright$ The CASE OF NON-SMOOTH $h$. The previous derivation which involves $h^{\prime}, h^{\prime \prime}$ and possibly higher derivatives is mathematically incorrect if $h$ is not smooth. This fact is clear using the stochastic analysis approach. It is also clear using PDE arguments: indeed, it would involve the perturbed PDE solution $(t, x) \mapsto \mathbb{E}\left(h\left(Y_{T}^{\varepsilon}\right) \mid Y_{t}^{\varepsilon}=x\right)$, that is not uniformly smooth (because the regularization parameter $\varepsilon$ shrinks to 0 ). If $h(x)=\mathbf{1}_{x \geq K}$ (like digital payoff), i.e. we evaluate $p^{\varepsilon}=\mathbb{P}\left(Y_{T}^{\varepsilon} \geq K\right)$, and $y_{0, T} \neq K$, large deviation arguments [25] show that the probability $p^{\varepsilon}$ is exponentially close to 0 or 1 w.r.t. $1 / \varepsilon^{2}$ (i.e. $\log \left(p^{\varepsilon}\right) \approx-c / \varepsilon^{2}$ if $y_{0, T}<K$ ), and thus an expansion in power of $\varepsilon$ provides zero coefficients at any order. To get a non degenerate and 
interesting situation, we should consider the case $K$ is close to $y_{0, T}$ in the sense $K=y_{0, T}+\lambda \varepsilon$, that is

$$
p^{\varepsilon}=\mathbb{P}\left(Y_{T}^{\varepsilon} \geq y_{0, T}+\lambda \varepsilon\right)=\mathbb{P}\left(\frac{Y_{T}^{\varepsilon}-y_{0, T}}{\varepsilon} \geq \lambda\right) .
$$

In other words, to overcome the difficulty of the singularity of $h$, we have leveraged a homogenization argument (singular perturbation), by considering the rescaled variable (usually called fast variable) $Z_{t}^{\varepsilon}=\frac{Y_{t}^{\varepsilon}-y_{0, t}}{\varepsilon}=Y_{1, t}+\frac{1}{2} \varepsilon Y_{2, t}+o(\varepsilon)$. If the law of $Y_{1, T}$ is non degenerate (for instance Gaussian law with non-zero variance), the latter quantity can be expanded in powers of $\varepsilon$. Actually, for less specific functions $h$, Watanabe [26] has developed a Malliavin calculus-based machinery to establish a general expansion result of $\mathbb{E}\left(h\left(Z_{T}^{\varepsilon}\right)\right)$ in powers of $\varepsilon$, available even for Schwartz distributions $h$, assuming stochastic expansions in Malliavin sense of

$$
Z_{T}^{\varepsilon}=Z_{0, T}+\varepsilon Z_{1, T}+\varepsilon^{2} Z_{2, T}+\cdots+\varepsilon^{n} Z_{n, T}+O\left(\varepsilon^{n+1}\right)
$$

for any $n \geq 1$ and asymptotic (in $\varepsilon$ ) non-degeneracy in Malliavin sense of $Z_{T}^{\varepsilon}$ :

$$
\limsup _{\varepsilon \rightarrow 0}\left\|1 / \operatorname{det}\left(\gamma^{Z_{T}^{\varepsilon}}\right)\right\|_{p}<+\infty
$$

for any $p \geq 1$, where $\gamma^{Z}$ is the Malliavin covariance matrix of a random variable $Z$. The Watanabe result states the existence of random variables $\left(\pi_{k}\right)_{k \geq 1}$ such that for any polynomially bounded function $h$, we have

$$
\mathbb{E}\left(h\left(Z_{T}^{\varepsilon}\right)\right)=\mathbb{E}\left(h\left(Z_{0, T}\right)\right)+\sum_{k=1}^{n} \varepsilon^{k} \mathbb{E}\left(h\left(Z_{0, T}\right) \pi_{k}\right)+O\left(\varepsilon^{n+1}\right), \quad \forall n \geq 1 .
$$

Compared to the non-smooth case, the possibility to get an expansion result is due to the non-degeneracy condition which has a (asymptotic) regularization effect on the non-smooth function $h$. With our previous notation $Z_{T}^{\varepsilon}=\frac{Y_{T}^{\varepsilon}-y_{0, T}}{\varepsilon}=Y_{1, T}+\frac{1}{2} \varepsilon Y_{2, T}+$ $o(\varepsilon)$, the asymptotic non-degeneracy (18) implies that the Gaussian random variable $Y_{1, T}$ has a non-zero variance, i.e. $\int_{0}^{T} e^{2 \int_{s}^{T} \partial_{x} \mu\left(r, y_{0}, r\right) \mathrm{d} r} \nu^{2}\left(s, y_{0, s}\right) \mathrm{d} s>0$ : in the case of time-independent coefficient $\mu(s, y)=\mu(y), \nu(s, y)=\nu(y)$, it reads $\nu\left(y_{0, T}\right) \neq 0$. The converse result $\left(\nu\left(y_{0, T}\right) \neq 0\right.$ implies $\left.(18)\right)$ holds true in the case of time-independent coefficient and in a multidimensional setting, see [26, Theorem 3.4]. Yoshida [27, Theorem 2.2] has weakened the assumption (18) into a localized version allowing degeneracy on a set of polynomially small probability measure. This approach has been successfully applied to different pricing problems in finance, mainly by Yoshida, Takahashi and their co-authors: see [28, 29, 30] or the unpublished work [31]. Their methodology consists in expanding the density of the random variable $Z_{T}^{\varepsilon}=\frac{Y_{T}^{\varepsilon}-y_{0, T}}{\varepsilon}$ using the Gaussian density as the zero-order term, and then going back to $\mathbb{E}\left(h\left(Y_{T}^{\varepsilon}\right)\right)$ by suitable integration computations. The advantage of this approach is that the expansion result (19) holds in a large generality, provided that we assume infinitely differentiable coefficients and uniform non degeneracy. However, observe two difficulties or restrictions: 
- within usual financial models like Heston model, the required regularity assumption is not satisfied and we even know that the Malliavin differentiability of high order may fail, see [32].

- the existence of the Malliavin weights $\left(\pi_{k}\right)_{k}$ does not provide an explicit and numerically computable expansion: very involved additional computations are required to obtain explicit formulas. One might compare these tricky computations to those necessary to solve the aforementioned system of PDEs.

Last, this approach usually leads to normal approximations of financial models (Bachelier prices) whereas log-normal approximations (Black-Scholes prices) might be more accurate (numerical evidences are given in Section 7).

After this presentation of singular perturbation using stochastic analysis, we now turn to the PDE approach. It has been developed in the financial context by Hagan and co-authors $[5,33]$. To be as close as possible to the quoted work, assume that the drift coefficient is $\mu \equiv 0$. In the case of Call payoff, the original valuation PDE $u^{\varepsilon}$ writes

$$
\left\{\begin{array}{l}
\partial_{t} u^{\varepsilon}(t, x)+\frac{1}{2} \varepsilon^{2} \nu^{2}(t, x) \partial_{x}^{2} u^{\varepsilon}(t, x)=0 \quad \text { for } t<T, \\
u^{\varepsilon}(T, x)=(x-K)_{+} ;
\end{array}\right.
$$

now, if we consider ATM strikes ( $K-Y_{0}=O(\varepsilon)$ similarly to before), we should consider the fast variable $y=(x-K) / \varepsilon$ and the rescaled solution $v^{\varepsilon}(t, y)=$ $\frac{1}{\varepsilon} u^{\varepsilon}(t, K+\varepsilon y)$ which solves

$$
\left\{\begin{array}{l}
\partial_{t} v^{\varepsilon}(t, y)+\frac{1}{2} \nu^{2}(t, K+\varepsilon y) \partial_{y}^{2} v^{\varepsilon}(t, y)=0 \text { for } t<T, \\
v^{\varepsilon}(T, y)=y_{+}
\end{array}\right.
$$

At this stage, the analysis follows the routine similar to before, by seeking a solution under the form

$$
v^{\varepsilon}=v_{0}+\varepsilon v_{1}+o(\varepsilon)
$$

solving $\mathcal{L}^{\varepsilon} v^{\varepsilon}=0$ where $\mathcal{L}^{\varepsilon}=\partial_{t}+\frac{1}{2} \nu^{2}(t, K+\varepsilon y) \partial_{y}^{2}=\mathcal{L}_{0}+\varepsilon \mathcal{L}_{1}+o(\varepsilon)$ with $\mathcal{L}_{0}=\partial_{t}+\frac{1}{2} \nu^{2}(t, K) \partial_{y}^{2}, \mathcal{L}_{1}=\nu \nu^{\prime}(t, K) y \partial_{y}^{2} . \quad$ A formal identification leads to a system of PDEs:

$$
\mathcal{L}_{0} v_{0}=0, v_{0}(T, y)=y_{+} \quad \text { and } \quad \mathcal{L}_{0} v_{1}+\mathcal{L}_{1} v_{0}=0, v_{1}(T, y)=0 .
$$

The solution $v_{0}$ is obviously given by the Call price in a Bachelier model (8) $\mathrm{d} X_{t}^{\mathrm{BA}}=\nu(t, K) \mathrm{d} W_{t}$ with time-dependent diffusion coefficient, and the first correction is given by $v_{1}(t, y)=\mathbb{E}\left(\int_{t}^{T} \mathcal{L}_{1} v_{0}\left(s, X_{s}^{\mathrm{BA}}\right) \mathrm{d} s \mid X_{t}^{\mathrm{BA}}=y\right)$. Although the new terminal function $h(y)=y_{+}$is not infinitely smooth, non-zero function $\nu$ induces a smoothing effect due to a non-degenerate heat kernel (this feature is analogous to the previous non-degeneracy in the Malliavin sense): hence, $v_{0}$ is smooth with derivatives possibly blowing up as time gets close to $T$ and a careful analysis shows that $v_{1}$ is well defined too. Here again, the explicit computation of $v_{1}$ is not an easy 
exercise and it requires some tricks. Finally $v_{1}$ can be written as the weighted sum of derivatives of $v_{0}$ (interpreted as Greeks). In Sections 3 and 5 , we provide a more direct and generic way to compute this kind of correction terms using stochastic analysis instead of PDE arguments.

Regarding the careful justification of the above PDE regular expansion with error estimates, quite surprisingly we have not been able to find literature references when the terminal condition is non-smooth (like $y \mapsto y_{+}$).

Once obtained the expansion of $v^{\varepsilon}$ for a given local volatility function $\sigma(.,$.$) (i.e.$ $\nu(t, y)=y \sigma(t, y))$, one can derive an expansion of the Black-Scholes implied volatility $\sigma_{\mathrm{I}}$ by identifying the previous expansion with that in the case $\left(\nu_{\mathrm{I}}(t, y)=y \sigma_{\mathrm{I}}\right)$ : see [5] where the analysis is successfully performed for time-independent volatility $\sigma(t, y)=\sigma(y)$ (or separable function $\sigma(t, y)=\sigma(y) \alpha(t)$ by a simple time-change). It is possible that the case of general time-dependent volatility has been considered out of reach by the authors of $[5,33]$ using PDE arguments, whereas we will see later how much stochastic analysis tools are suitable even in the case time-dependent coefficients.

\subsubsection{Short maturity}

In this asymptotics, the terminal time $T$ is small. When one has to evaluate $\mathbb{E}\left(h\left(Y_{T}\right)\right)$ for $Y$ solution of the SDE (14) and for $h$ smooth (say infinitely differentiable with bounded derivatives), iterative applications of Itô's formula give

$$
\begin{aligned}
\mathbb{E}\left(h\left(Y_{T}\right)\right) & =h\left(Y_{0}\right)+\int_{0}^{T} \mathbb{E}\left([\mathcal{L} h]\left(t, Y_{t}\right)\right) \mathrm{d} t \\
& =h\left(Y_{0}\right)+T[\mathcal{L} h]\left(0, Y_{0}\right)+\int_{0}^{T} \int_{0}^{t} \mathbb{E}\left(\left[\mathcal{L}^{2} h\right]\left(s, Y_{s}\right)\right) \mathrm{d} s \mathrm{~d} t
\end{aligned}
$$

where $\mathcal{L}$ is the infinitesimal generator associated to $Y$. Iterating the procedure, we obtain an expansion in powers of $T$ :

$$
\mathbb{E}\left(h\left(Y_{T}\right)\right)=\sum_{k=0}^{n} \frac{T^{k}}{k !}\left[\mathcal{L}^{k} h\right]\left(0, Y_{0}\right)+O\left(T^{n+1}\right), \quad n \geq 0 .
$$

The numerical evaluation of such formula is straightforward. We refer the reader to [34, Chap. 5] for a more comprehensive exposure of related Itô-Taylor expansions.

As in the case of small noise expansion, the case of non-smooth $h$ requires a different treatment because $\mathcal{L}^{k} h$ is not defined. For this, we transform the problem of small terminal time with fixed coefficients into a problem of fixed terminal time with small coefficients, by leveraging the scaling property of the Brownian motion. Actually, having $T$ small is equivalent to replace $T$ by $\varepsilon^{2} T$ with $\varepsilon \rightarrow 0$ : then, starting from the SDE (14), we consider the time-rescaled process $\left(Y_{\varepsilon^{2} t}\right)_{0 \leq t \leq T}$ which has the same distribution as $\left(Y_{t}^{\varepsilon}\right)_{0 \leq t \leq T}$ defined as the solution of

$$
\mathrm{d} Y_{t}^{\varepsilon}=\varepsilon^{2} \mu\left(\varepsilon^{2} t, Y_{t}^{\varepsilon}\right) \mathrm{d} t+\varepsilon \nu\left(\varepsilon^{2} t, Y_{t}^{\varepsilon}\right) \mathrm{d} W_{t}, \quad Y_{0}^{\epsilon}=Y_{0},
$$


see [26, p.17]. Observe that this leads to a different parameterization compared to the small noise case (in particular, the drift coefficient is multiplied by $\varepsilon^{2}$ ). However the expansion methodology is similar: in the case of non-smooth function, it is more appropriate to rescale the process by setting $Z_{t}^{\varepsilon}=\frac{Y_{t}^{\varepsilon}-y_{0, t}}{\varepsilon}=Y_{1, t}+\frac{1}{2} \varepsilon Y_{2, t}+o(\varepsilon)$, where

$$
\begin{aligned}
Y_{1, t}=\left.\partial_{\varepsilon} Y_{t}^{\varepsilon}\right|_{\varepsilon=0} & =\nu\left(0, Y_{0}\right) W_{t}, \\
Y_{2, t}=\left.\partial_{\varepsilon}^{2} Y_{t}^{\varepsilon}\right|_{\varepsilon=0} & =2 \mu\left(0, Y_{0}\right) t+2 \partial_{y} \nu\left(0, Y_{0}\right) \int_{0}^{t} Y_{1, s} \mathrm{~d} W_{s} \\
& =2 \mu\left(0, Y_{0}\right) t+\partial_{y} \nu\left(0, Y_{0}\right) \nu\left(0, Y_{0}\right)\left(W_{t}^{2}-t\right) .
\end{aligned}
$$

Once the fast variable is selected, observe that we are reduced to a regular perturbation problem, that can be handled using stochastic analysis tools (namely Watanabe approach [26]) or using PDE tools. We skip details since it is similar to what have been presented before. See also the book by Henry-Labordère [6], where short-time asymptotics of density functions are derived through geometry considerations.

\subsubsection{Fast volatility}

Since the end of the nineties, another popular approximation approach has been developed by Fouque, Papanicolaou and Sircar, see [35, 36]. It emphasizes that the asset volatility $\left(\sigma_{t}\right)_{t \geq 0}$ has usually slow variations compared to the variations of the asset itself (multiscale modeling). This is achieved in two different ways.

- Either the natural time scale of stochastic volatility is short, which leads to a model of the form (22) for $\left(\sigma_{t}\right)_{t}$, while the asset dynamics is unchanged. Thus, at order zero, we obtain a Black-Scholes model with a constant volatility equal to the initial stochastic volatility $\sigma_{0}$, see $[35$, Section 2].

- Or the fluctuations of the stochastic volatility $\left(\sigma_{t}\right)_{t}$ are so fast that they give the appearance of a constant (in time) volatility, when considered at a longer time scale. This second point of view has been much developed by Fouque, Papanicolaou and Sircar and their co-authors, in many respects, and this is presented below.

As an illustration of their methodology, we consider the asset model $\mathrm{d} S_{t}=S_{t} \sigma_{t} \mathrm{~d} W_{t}$ and an Ornstein-Uhlenbeck process for modeling $\left(\sigma_{t}=f\left(\Sigma_{t}\right)\right)_{t}$ with

$$
\mathrm{d} \Sigma_{t}=\frac{1}{\varepsilon}\left(\bar{\Sigma}_{\infty}-\Sigma_{t}\right) \mathrm{d} t+v \sqrt{\frac{2}{\varepsilon}} \mathrm{d} B_{t}
$$

For instance in the Scott model [37], $f(x)=e^{x}$ and $(W, B)$ is a standard bidimensional correlated Brownian motion $\left(\mathrm{d}\langle W, B\rangle_{t}=\rho \mathrm{d} t\right)$. As time goes to infinity, the random variable $\Sigma_{t}$ weakly converges to the stationary Gaussian distribution with mean $\bar{\Sigma}_{\infty}$ and variance $\left(v \sqrt{\frac{2}{\varepsilon}}\right)^{2} /\left(2 \frac{1}{\varepsilon}\right)=v^{2}$. In other words, although the fluctuations are fast (the characteristic time being $\varepsilon$ ), the distribution remains the 
same (at least for time larger than $\varepsilon$ ). It allows the application of ergodic theorem to obtain large-time asymptotics of integrals of the realized volatility: for any polynomially bounded function $\Psi$, we have

$$
\lim _{T \rightarrow+\infty} \frac{1}{T} \int_{0}^{T} \Psi\left(\sigma_{s}\right) \mathrm{d} s=\int_{\mathbb{R}} \Psi\left(e^{y}\right) \frac{1}{\sqrt{2 \pi v^{2}}} e^{-\left(y-\bar{\Sigma}_{\infty}\right)^{2} /\left(2 v^{2}\right)} \mathrm{d} y:=\sigma_{B S}^{2}
$$

in the almost sure sense and in the $L_{1}$ sense. For $\Psi(y)=y^{2}$, we obtain a constant large-time approximation of $\frac{1}{T} \int_{0}^{T} \sigma_{s}^{2} \mathrm{~d} s$ to be used as a zero-order approximation in a Black-Scholes formula. To derive correction terms, the authors employ singular pertubation PDE techniques: indeed, the price function $u^{\varepsilon}(t, x, y)=\mathbb{E}\left(h\left(S_{T}\right) \mid S_{t}=\right.$ $\left.x, \Sigma_{t}=y\right)$ solves $\mathcal{L}^{\varepsilon} u^{\varepsilon}=0$ with

$$
\begin{aligned}
\mathcal{L}^{\epsilon} & =\partial_{t}+\frac{1}{2} x^{2} f^{2}(y) \partial_{x x}^{2}+\sqrt{\frac{2}{\varepsilon}} \rho v x f(y) \partial_{x y}^{2}+\frac{1}{\varepsilon}\left(v^{2} \partial_{y}^{2}+\left(\bar{\Sigma}_{\infty}-y\right) \partial_{y}\right) \\
& :=\mathcal{L}_{0}+\frac{1}{\sqrt{\varepsilon}} \mathcal{L}_{1}+\frac{1}{\varepsilon} \mathcal{L}_{2} .
\end{aligned}
$$

As in the previous approaches, by decomposing $u^{\varepsilon}$ in powers of $\sqrt{\varepsilon}$ and by gathering the contributions of the same order, we obtain a system of PDEs characterizing the main order term and the correction terms. Actually the analysis is quite intricate because one has to take into account the ergodic property of $\sigma$ (which leads to solving elliptic PDEs of the form of Poisson equation): see [36, Chapter 5] where the error analysis is made for smooth payoffs and [38] for the Call option case. The final approximation pricing formula writes

$$
\begin{aligned}
& \mathbb{E}\left(S_{T}-K\right)_{+}=\mathrm{Call}^{\mathrm{BS}}\left(\log \left(S_{0}\right), T \sigma_{B S}^{2}, \log (K)\right) \\
& \quad+\sqrt{\varepsilon} \times \operatorname{linear} \text { combination of } \partial_{S}^{i} \mathrm{Call}^{\mathrm{BS}}\left(\log \left(S_{0}\right), T \sigma_{B S}^{2}, \log (K)\right) \text { for } i=2,3+\ldots
\end{aligned}
$$

with some explicit coefficients as the factors for the Greeks. Consequently, the approximation formula is straightforward to evaluate on a computer since BlackScholes price and Greeks are known in closed form and available in any pricing software. In this analysis and similarly to any PDE approaches, assuming time homogeneous coefficients simplifies much the derivation of explicit formula. In the context of fast volatility, some extensions are possible, see [39].

\subsubsection{Proxy expansion}

We complete our overview section by presenting a different point of view, which is going to be developed further in the next sections. As a difference with previous works, this is rather a non-asymptotic approach, relying on the a priori knowledge of proxy of the model to handle; for this reason, it may appear as more understandable and more intuitive for practioners. Consider the model (2) on $S$ :

$$
\mathrm{d} S_{t}=S_{t} \sigma\left(t, S_{t}\right) \mathrm{d} W_{t},
$$


and assume that by expertise, $S$ behaves closely to a Gaussian model, i.e. the fluctuations of $S_{t} \sigma\left(t, S_{t}\right)$ are small. Then, it is reasonable to take the Bachelier model $S^{P}$ with parameter $\left(\Sigma_{t}=S_{0} \sigma\left(t, S_{0}\right)\right)_{t}$ as a proxy, that is

$$
\mathrm{d} S_{t}^{P}=\Sigma_{t} \mathrm{~d} W_{t}, \quad S_{0}^{P}=S_{0} . \quad \text { (Normal Proxy) }
$$

The Call price in $S$ model should be close to that in the proxy; since this approximation may be too crude, it is recommended to add correction terms.

Alternatively, one could guess that $S$ rather behaves as a log-normal model with parameter $\left(a_{t}\right)_{t}$, i.e. $X=\log (S)$ may be approximated by

$$
\mathrm{d} X_{t}^{P}=-\frac{1}{2} a_{t}^{2} \mathrm{~d} t+a_{t} d W_{t}, \quad X_{0}^{P}=x_{0} . \quad \text { (Log-Normal Proxy) }
$$

The proxy volatility may be taken to $a_{t}=a\left(t, x_{0}\right)=\sigma\left(t, S_{0}\right)$ for instance, but another point could be chosen (for instance, the strike $K$ or the mid-point $(K+$ $\left.S_{0}\right) / 2$ ). This description does not put an emphasize on a specific asymptotic, but one has to quantify how $S_{t} \sigma\left(t, X_{t}\right) \approx \Sigma_{t}$ or $a\left(t, X_{t}\right) \approx a_{t}$.

To compute correction terms to the relation $\mathbb{E}\left(S_{T}-K\right)_{+} \approx \mathbb{E}\left(S_{T}^{P}-K\right)_{+}$or $\mathbb{E}\left(e^{X_{T}^{P}}-K\right)_{+}$, it is necessary to derive a convenient representation of the distance to the proxy $S_{T}-S_{T}^{P}$ or $X_{T}-X_{T}^{P}$. The linear interpolation $X_{T}^{\eta}=X_{T}^{P}+\eta\left(X_{T}-X_{T}^{P}\right)$ does not lead to illuminating computations. It is much better to consider the following interpolation: for $\eta \in[0,1]$, define

$$
d X_{t}^{\eta}=\eta\left(-\frac{1}{2} a^{2}\left(t, X_{t}^{\eta}\right) d t+a\left(t, X_{t}^{\eta}\right) d W_{t}\right), \quad X_{0}^{\eta}=x_{0} .
$$

Note that $\eta$ is not a small parameter but an interpolation parameter. Observe also that this parameterization is different from that in small time or small noise asymptotics.

A direct computation shows that $X_{t}^{1}=X_{t}, X_{t}^{0}=x_{0}$ and $\left.\partial_{\eta} X_{t}^{\eta}\right|_{\eta=0}=$ $\int_{0}^{t} a\left(s, x_{0}\right)\left[\mathrm{d} W_{s}-\frac{1}{2} a\left(s, x_{0}\right) \mathrm{d} s\right]$ : this shows that $X_{t}-X_{t}^{P}=X_{t}^{1}-\left(X_{t}^{0}+\left.\partial_{\eta} X_{t}^{\eta}\right|_{\eta=0}\right)$ writes as a Taylor formula at order 1 . Thus, the natural candidate for the first contribution in $X_{t}-X_{t}^{P}$ is $\left.\frac{1}{2} \partial_{\eta}^{2} X_{t}^{\eta}\right|_{\eta=0}$. The above interpolation is equivalent to

$$
d \hat{X}_{t}^{\eta}=-\frac{1}{2} a^{2}\left(t, x_{0}+\eta\left(\hat{X}_{t}^{\eta}-x_{0}\right)\right) d t+a\left(t, x_{0}+\eta\left(\hat{X}_{t}^{\eta}-x_{0}\right)\right) d W_{t}, \quad \hat{X}_{0}^{\eta}=x_{0},
$$

which is related to $X_{t}^{\eta}$ by the relation $X_{t}^{\eta}=x_{0}+\eta\left(\hat{X}_{t}^{\eta}-x_{0}\right)$.

\subsection{Asymptotic expansion versus non-asymptotic expansion}

Deriving an asymptotic expansion sheds the light on the crucial role of one model parameter compared to the other ones, to explain and approximate the option prices: for instance, in small noise expansion, we focus only on the volatility by putting an $\varepsilon$ in front of the $\mathrm{d} W$-term, and so on. It finally leads to a generic expansion of the form

$$
u^{\varepsilon}=u_{0}+\varepsilon u_{1}+\frac{1}{2} \varepsilon^{2} u_{2}+\ldots
$$


or in powers of $\sqrt{\varepsilon}$ in the fast volatility framework. Our previous discussion has shown how this expansion is obtained in a Markovian framework using PDE (regular or singular pertubations), or more generally using Malliavin calculus (Watanabe approach).

Actually, it is important to observe that writing such an expansion implicitly means that apart of the parameter related to $\varepsilon$, the other parameters have no important asymptotics for the problem under consideration. Below we consider a simple toy example to show that there might be a competition between all the model parameters, and moreover there is a necessary trade-off with the payoff regularity. In other words, deriving (25) does not necessarily mean that the first order approximation $u_{0}+\varepsilon u_{1}$ is really accurate and taking more terms do not necessarily improve the accuracy, because of the possible crucial influence of other large or small parameters. Our toy example is the following perturbed Brownian model:

$$
X_{1}^{\varepsilon}=\sigma W_{1}+\sqrt{\varepsilon} B_{1}
$$

where $(W, B)$ is a two-dimensional Brownian motion, and $\sigma$ is positive. This toy model can be viewed as the simplest way to perturb a volatility model (we could have taken $B=W$ without changing the conclusion of the discussion below) and thus, it is quite realistic compared to the further situations to handle.

(1) Case $h(x)=1+x^{2}$. We have $\mathbb{E}\left[h\left(X_{1}^{\varepsilon}\right)\right]=1+\sigma^{2}+\varepsilon=\mathbb{E}\left[h\left(X_{1}^{0}\right)\right]+\varepsilon$.

(2) Case $h(x)=1+x_{+}$. By a scaling argument, we have:

$$
\mathbb{E}\left[h\left(X_{1}^{\varepsilon}\right)\right]=1+\sqrt{\sigma^{2}+\varepsilon} \mathbb{E}\left[\left(W_{1}\right)^{+}\right]=\mathbb{E}\left[h\left(X_{1}^{0}\right)\right]+\frac{1}{2} \frac{\varepsilon}{\sigma} \mathbb{E}\left[\left(W_{1}\right)^{+}\right]+O\left(\frac{\varepsilon^{2}}{\sigma^{3}}\right) .
$$

(3) Case $h(x)=1_{x \leq x_{0}}$. We have:

$$
\mathbb{E}\left[h\left(X_{1}^{\varepsilon}\right)\right]=\mathcal{N}\left(\frac{x_{0}}{\sqrt{\sigma^{2}+\varepsilon}}\right)=\mathcal{N}\left(\frac{x_{0}}{\sigma}\right)-\mathcal{N}^{\prime}\left(\frac{x_{0}}{\sigma}\right) \frac{x_{0}}{\sigma} \frac{\varepsilon}{2 \sigma^{2}}+O\left(\frac{\varepsilon^{2}}{\sigma^{4}}\right) .
$$

These simple computations show that the expansion order depends on the relative magnitude of $\varepsilon$ and $\sigma$, and also on the regularity of the function $h$. For instance, if $\sigma$ is also small, say $\varepsilon=\sigma^{3} \rightarrow 0$, then the expansion order w.r.t. $\sigma$ in the case (1), (2), (3) is respectively equal to 3,2 and 1 . These subtleties do not appear in the expansions (19) of Watanabe type or (21) of PDE type, because the focus is made only on a single small parameter $\varepsilon$.

It means that in some situations, asymptotic expansions may be misleading or may not give the best possible approximations; then, we should take into account the influence of all (or many) model parameters. In the context of fast volatility, multi-scale modeling and its related asymptotic analysis are very recently developed in [24, Chapter 4]; see also [40, Chapter 3 and Section 4.4].

In the sequel of this work, we consider non asymptotic expansions, mainly for local volatility models, and analyse the approximation error taking into account several parameters at the same time, in order to determine in which extent they play 
complementary or opposite roles. For instance, it is informative to see the simultaneous influences of maturity, of volatility amplitude or of derivatives of volatility function on the option prices. Their impacts depend on the payoff smoothness: the accuracy is expected to be improved for smooth payoff compared to non-smooth payoffs.

Final considerations. After this (hopefully complete) overview, the reader may wonder what is the best approximation method among those presented. Of course, it depends on the required accuracy and the computational time allowed for the numerical evaluation. From this point of view, all methods are not equivalent. The choice of relevant asymptotics/approximations guarantees to catch the main features of the pricing problem, and as a consequence, it will likely lead to an expansion of low order to achieve a good accuracy (with low computational time or complexity). In these respects, the proxy expansion has immediate advantages: the better or the more intuitive the proxy, the smaller the number of correction terms.

One should also take care of the preservation of some model properties in the approximation.

- One of them is the martingale property of $S=e^{X}$ (serving as a base for Call/Put parity relation). For instance, a small noise approximation of $X$ defined in (3) does not maintain the martingale property since the volatility coefficient is scaled by $\varepsilon$ while the drift remains unchanged: as a result, the final approximation may suffer from numerical arbitrage.

- Another property is positivity of $S$. Taking a Normal Proxy for $S$ may give wrong results if the values of $S$ close to 0 have a prominent role in the computation of $\mathbb{E}\left(h\left(S_{T}\right)\right)$ (for instance, Call/Put with small strikes).

These kinds of consideration may help to choose between different methods, with the additional help of comparative numerical tests.

\section{Approximation based on proxy}

\subsection{Notations and definitions}

The following notations and definitions are repeatedly used in this work.

$\triangleright$ Differentiation. If these derivatives have a meaning, we write $l_{t}^{(i)}(x)=\partial_{x^{i}}^{i} l(t, x)$ for any function $l$ of two variables.

$\triangleright$ Integral Operator. The integral operator $\omega^{T}$ is defined as follows: for any measurable and bounded function $l$, we set

$$
\omega(l)_{t}^{T}=\int_{t}^{T} l_{u} d u
$$


for $t \in[0, T]$. Its $n$-times iteration is defined analogously: for any measurable and bounded functions $\left(l_{1}, \cdots, l_{n}\right)$, we set

$$
\omega\left(l_{1}, \cdots, l_{n}\right)_{t}^{T}=\omega\left(l_{1} \omega\left(l_{2}, \cdots, l_{n}\right)_{.}^{T}\right)_{t}^{T},
$$

for $t \in[0, T]$.

$\triangleright$ Time reversal. For any measurable and bounded function $l$, we denote by $\widetilde{l}$ the function $\widetilde{l}_{t}=l_{T-t}$ for any $t \in[0, T]$. Notice the relation

$$
\omega\left(\widetilde{l}_{1}, \widetilde{l}_{2}, . ., \widetilde{l}_{n}\right)_{0}^{T}=\omega\left(l_{n}, l_{n-1}, . ., l_{1}\right)_{0}^{T}
$$

available for any measurable and bounded functions $\left(l_{1}, \cdots, l_{n}\right)$ : in other words, reversing the time of integrands is equivalent to change the order of integration.

$\triangleright$ Quadratic mean on $[0, T]$. For any measurable function $(l(t, x))_{(t, x) \in[0, T] \times \mathbb{R}}$ of two variables, bounded w.r.t. the time variable for any $x \in \mathbb{R}$, we denote by $\bar{l}_{z}$ its quadratic mean on $[0, T]$ at the spatial point $z$ defined by:

$$
\bar{l}_{z}=\sqrt{\frac{1}{T} \int_{0}^{T} l_{t}^{2}(z) \mathrm{d} t .}
$$

This notation is frequently used for the function $a$ at the points $z=x_{0}, k, x_{\mathrm{av}}$ and for the function $\Sigma$ at $z=S_{0}, K, S_{\mathrm{av}}$.

\section{$\triangleright$ Assumptions on $a$ and $\Sigma$.}

- $\left(\mathcal{H}^{a}\right): a$ is a bounded measurable function of $(t, x) \in[0, T] \times \mathbb{R}$, and five times continuously differentiable in $x$ with bounded $^{\mathrm{d}}$ derivatives. Set $^{-}$

$\mathcal{M}_{1}(a)=\max _{1 \leq i \leq 5} \sup _{(t, x) \in[0, T] \times \mathbb{R}}\left|\partial_{x}^{i} a(t, x)\right|$ and $\mathcal{M}_{0}(a)=\max _{0 \leq i \leq 5} \sup _{(t, x) \in[0, T] \times \mathbb{R}}\left|\partial_{x}^{i} a(t, x)\right|$.

In addition, there exists a constant $c_{a}>0$ such that $|a(t, x)| \geq c_{a}$ for any $(t, x) \in[0, T] \times \mathbb{R}$

- $\left(\mathcal{H}_{z}^{a}\right)$ : assume $\left(\mathcal{H}^{a}\right)$ by replacing the last uniform ellipticity by the single condition $\int_{0}^{T}|a(t, z)|^{2} \mathrm{~d} t>0$

The above hypothesis will be considered at $z=x_{0}, z=k$ or $z=x_{\mathrm{av}}$.

We define similarly $\left(\mathcal{H}^{\Sigma}\right)$ or $\left(\mathcal{H}_{z}^{\Sigma}\right)$ by replacing $a$ by $\Sigma$ in $\left(\mathcal{H}^{a}\right)$ and $\left(\mathcal{H}_{z}^{a}\right)$. Then the hypothesis will be considered at $z=S_{0}, z=K$ or $z=S_{\mathrm{av}}$.

$\triangleright$ Constants. Our next error estimates are stated following the notation below.

- " $A=\mathcal{O}(B) "$ means that $|A| \leq C B$ : here, $C$ stands for a generic constant that is a non-negative increasing function of $T, \mathcal{M}_{1}(a), \mathcal{M}_{0}(a)$ and of the oscillation ratio $\frac{\mathcal{M}_{0}(a)}{c_{a}}$ (if $\left(\mathcal{H}^{a}\right)$ is fulfilled) or $\frac{\left[\mathcal{M}_{0}(a)\right]^{2} T}{\int_{0}^{T}|a(t, z)|^{2} \mathrm{~d} t}$ (if $\left(\mathcal{H}_{z}^{a}\right)$ is fulfilled).

If $\left(\mathcal{H}^{\Sigma}\right)$ or $\left(\mathcal{H}_{z}^{\Sigma}\right)$ is satisfied, in the above dependence $a$ has to replaced by $\Sigma$.

dhe boundedness assumption of $a$ and its derivatives could be weakened to $L_{p}$-integrability conditions, up to extra works. 
Usually, a generic constant may depend on $S_{0}, x_{0}, K$ and $k$; nevertheless, it remains uniformly bounded in these variables: it is possible to derive exact dependency but we skip it to keep the analysis short.

- Similarly, if $A$ is positive, $A \leq_{c} B$ means that $A \leq C B$ for a generic constant C.

\subsection{Proxy approximation: a primer using the local volatility at spot}

$\triangleright$ Log-normal proxy. Assume by expertise that the model (2) behaves closely to a log-normal model, in the sense that a log-normal approximation seems to be reasonable. For instance, in the case of CEV type model

$$
S \sigma(t, S)=\nu_{t} S^{\beta_{t}},
$$

a log-normal heuristics is associated to $\beta$ close to 1 . Some numerical illustrations are given later.

As a first log-normal approximation, we freeze the volatility in space to the initial spot value: regarding the log-asset $X$ defined in (3), it writes

$$
\mathrm{d} X_{t}^{P}=-\frac{1}{2} a^{2}\left(t, x_{0}\right) \mathrm{d} t+a\left(t, x_{0}\right) \mathrm{d} W_{t}, \quad X_{0}^{P}=x_{0} .
$$

We refer to this proxy model as log-normal proxy with volatility at spot. The evaluation of the next correction terms requires a suitable representation of the distance between the model and the proxy: for this, we use the interpolated process (23) given by

$$
d X_{t}^{\eta}=\eta\left(-\frac{1}{2} a^{2}\left(t, X_{t}^{\eta}\right) \mathrm{d} t+a\left(t, X_{t}^{\eta}\right) \mathrm{d} W_{t}\right), \quad X_{0}^{\eta}=x_{0} .
$$

for an interpolation parameter $\eta \in[0,1]$. Under $\left(\mathcal{H}_{x_{0}}^{a}\right)$, the three first derivatives of $\eta \mapsto X_{t}^{\eta}$ are well defined (a.s. simultaneously for any $t$, see [41, Theorem 2.3]). Denote by $X_{i, t}^{\eta}$ and $X_{i, t}$ the $i$-th derivative respectively at $\eta$ and $\eta=0$. Direct computations yield

$$
\begin{aligned}
d X_{1, t}^{\eta}= & -\frac{1}{2} a^{2}\left(t, X_{t}^{\eta}\right) d t+a\left(t, X_{t}^{\eta}\right) d W_{t} \\
& +\eta X_{1, t}^{\eta}\left(-\left[a \partial_{x} a\right]\left(t, X_{t}^{\eta}\right) \mathrm{d} t+\partial_{x} a\left(t, X_{t}^{\eta}\right) \mathrm{d} W_{t}\right), \quad X_{1,0}^{\eta}=0 . \\
d X_{2, t}^{\eta}= & 2 X_{1, t}^{\eta}\left(-\left[a \partial_{x} a\right]\left(t, X_{t}^{\eta}\right) \mathrm{d} t+\partial_{x} a\left(t, X_{t}^{\eta}\right) \mathrm{d} W_{t}\right) \\
& +\eta X_{2, t}^{\eta}\left(-\left[a \partial_{x} a\right]\left(t, X_{t}^{\eta}\right) \mathrm{d} t+\partial_{x} a\left(t, X_{t}^{\eta}\right) \mathrm{d} W_{t}\right) \\
& +\eta\left[X_{1, t}^{\eta}\right]^{2}\left(-\partial_{x}\left[a \partial_{x} a\right]\left(t, X_{t}^{\eta}\right) \mathrm{d} t+\partial_{x}^{2} a\left(t, X_{t}^{\eta}\right) \mathrm{d} W_{t}\right), \quad X_{2,0}^{\eta}=0 . \\
d X_{3, t}^{\eta}= & 3 X_{2, t}^{\eta}\left(-\left[a \partial_{x} a\right]\left(t, X_{t}^{\eta}\right) \mathrm{d} t+\partial_{x} a\left(t, X_{t}^{\eta}\right) \mathrm{d} W_{t}\right) \\
& +3\left[X_{1, t}^{\eta}\right]^{2}\left(-\partial_{x}\left[a \partial_{x} a\right]\left(t, X_{t}^{\eta}\right) \mathrm{d} t+\partial_{x}^{2} a\left(t, X_{t}^{\eta}\right) \mathrm{d} W_{t}\right), \\
& +\eta X_{3, t}^{\eta}\left(-\left[a \partial_{x} a\right]\left(t, X_{t}^{\eta}\right) \mathrm{d} t+\partial_{x} a\left(t, X_{t}^{\eta}\right) \mathrm{d} W_{t}\right) \\
& +3 \eta\left[X_{1, t}^{\eta}\right]\left[X_{2, t}^{\eta}\right]\left(-\partial_{x}\left[a \partial_{x} a\right]\left(t, X_{t}^{\eta}\right) \mathrm{d} t+\partial_{x}^{2} a\left(t, X_{t}^{\eta}\right) \mathrm{d} W_{t}\right) \\
& +\eta\left[X_{1, t}^{\eta}\right]^{3}\left(-\partial_{x}^{2}\left[a \partial_{x} a\right]\left(t, X_{t}^{\eta}\right) \mathrm{d} t+\partial_{x}^{3} a\left(t, X_{t}^{\eta}\right) \mathrm{d} W_{t}\right), \quad X_{3,0}^{\eta}=0 .
\end{aligned}
$$


Observe that $\left.X_{t}^{\eta}\right|_{\eta=0}=x_{0}$, thus the derivatives at $\eta=0$ have simpler expressions:

$$
\begin{aligned}
& d X_{1, t}=-\frac{1}{2} a^{2}\left(t, x_{0}\right) \mathrm{d} t+a\left(t, x_{0}\right) \mathrm{d} W_{t}=d X_{t}^{P}, \\
& d X_{2, t}=2 X_{1, t}\left(-\left[a \partial_{x} a\right]\left(t, x_{0}\right) \mathrm{d} t+\partial_{x} a\left(t, x_{0}\right) \mathrm{d} W_{t}\right),
\end{aligned}
$$

with $X_{i, 0}=0$ for $i \geq 1$. Then notice that $X_{t}^{P}=x_{0}+X_{1, t}$ : hence

$$
\begin{aligned}
X_{T}-X_{T}^{P}=X_{T}^{1}-\left(x_{0}+X_{1, T}\right) & =\int_{0}^{1}(1-\lambda) X_{2, T}^{\lambda} \mathrm{d} \lambda \\
& =\frac{1}{2} X_{2, T}+\int_{0}^{1} \frac{(1-\lambda)^{2}}{2} X_{3, T}^{\lambda} \mathrm{d} \lambda
\end{aligned}
$$

using the Taylor expansion formula. As a consequence of the above representation, we obtain an approximation of $\mathbb{E}\left(h\left(X_{T}\right)\right)$ for a smooth function $h$ :

$$
\mathbb{E}\left[h\left(X_{T}\right)\right]=\mathbb{E}\left[h\left(X_{T}^{P}+\frac{X_{2, T}}{2}+\ldots\right)\right]=\mathbb{E}\left[h\left(X_{T}^{P}\right)\right]+\mathbb{E}\left[h^{(1)}\left(X_{T}^{P}\right) \frac{X_{2, T}}{2}\right]+\ldots
$$

The first term is related to a log-normal model and thus, it is expected to be easily computable numerically. The second term is more delicate: actually, we transform it into a weighed sum of sensitivities of $\mathbb{E}\left[h\left(X_{T}^{P}+\varepsilon\right)\right]$ w.r.t. $\varepsilon=0$. To achieve this transformation, we use a key lemma which proof is given in Subsection 8.4

Lemma 4. Let $\varphi$ be a $C_{b}^{\infty}$ function and $\left(\lambda_{t}\right)_{t}$ be a measurable and bounded deterministic function. Let $N \geq 1$ be fixed, and consider measurable and bounded deterministic functions $t \mapsto l_{i, t}$ for $i=1, \ldots, N$. Then, using the convention $\mathrm{d} W_{t}^{1}=\mathrm{d} W_{t}$ and $\mathrm{d} W_{t}^{0}=\mathrm{d} t$, for any $\left(I_{1}, \ldots, I_{N}\right) \in\{0,1\}^{N}$ we have:

$$
\begin{aligned}
& \mathbb{E}\left(\varphi\left(\int_{0}^{T} \lambda_{t} \mathrm{~d} W_{t}\right) \int_{0}^{T} l_{N, t_{N}} \int_{0}^{t_{N}} l_{N-1, t_{N}-1} \ldots \int_{0}^{t_{2}} l_{1, t_{1}} \mathrm{~d} W_{t_{1}}^{I_{1}} \ldots \mathrm{d} W_{t_{N-1}}^{I_{N-1}} \mathrm{~d} W_{t_{N}}^{I_{N}}\right) \\
& =\left.\omega\left(\widehat{l}_{1}, \ldots, \widehat{l}_{N}\right) \partial_{\varepsilon^{I_{1}+\cdots+I_{N}}}^{I_{1}+\cdots+I_{N}} \mathbb{E}\left(\varphi\left(\int_{0}^{T} \lambda_{t} \mathrm{~d} W_{t}+\varepsilon\right)\right)\right|_{\varepsilon=0},
\end{aligned}
$$

where $\widehat{l}_{k, t}:=l_{k, t}$ if $I_{k}=0$ and $\widehat{l}_{k, t}:=\lambda_{t} l_{k, t}$ if $I_{k}=1$.

Now, apply the above identity to $\varphi(\cdot)=h^{(1)}\left(x_{0}-\frac{1}{2} \int_{0}^{T} a^{2}\left(t, x_{0}\right) \mathrm{d} t+\cdot\right), \lambda_{t}=a\left(t, x_{0}\right)$ and

$$
\begin{aligned}
\frac{X_{2, T}}{2}=\int_{0}^{T}\left(\int_{0}^{t_{2}}(-\right. & \left.\left.\frac{1}{2} a^{2}\left(t_{1}, x_{0}\right) \mathrm{d} t_{1}+a\left(t_{1}, x_{0}\right) \mathrm{d} W_{t_{1}}\right)\right) \\
& \times\left(-\left[a \partial_{x} a\right]\left(t_{2}, x_{0}\right) \mathrm{d} t_{2}+\partial_{x} a\left(t_{2}, x_{0}\right) \mathrm{d} W_{t_{2}}\right),
\end{aligned}
$$

to get

$$
\mathbb{E}\left[h^{(1)}\left(X_{T}^{P}\right) \frac{X_{2, T}}{2}\right]=\left.C_{1}\left(a ; x_{0}\right)_{0}^{T}\left(\partial_{\varepsilon^{3}}^{3}-\frac{3}{2} \partial_{\varepsilon^{2}}^{2}+\frac{1}{2} \partial_{\varepsilon}\right) \mathbb{E}\left(h\left(X_{T}^{P}+\varepsilon\right)\right)\right|_{\varepsilon=0}
$$

where the operator $C_{1}$ is defined by:

$$
C_{1}(l ; z)_{0}^{T}=\omega\left(l^{2}(z), l(z) l^{(1)}(z)\right)_{0}^{T}=\int_{0}^{T} l_{t}^{2}(z) \int_{t}^{T} l_{s}(z) l_{s}^{(1)}(z) \mathrm{d} s \mathrm{~d} t .
$$


Combine this with (33) to obtain that $\mathbb{E}\left(h\left(X_{T}\right)\right)$ can be approximated by

$$
\mathbb{E}\left[h\left(X_{T}^{P}\right)\right]+\left.C_{1}\left(a ; x_{0}\right)_{0}^{T}\left(\partial_{\varepsilon^{3}}^{3}-\frac{3}{2} \partial_{\varepsilon^{2}}^{2}+\frac{1}{2} \partial_{\varepsilon}\right) \mathbb{E}\left(h\left(X_{T}^{P}+\varepsilon\right)\right)\right|_{\varepsilon=0} .
$$

So far, the payoff function $h$ is smooth and this does not fit the Call/Put setting; actually, an extra regularization argument and a careful passing to the limit enables to extend the previous formula to any locally Lipschitz $h$. Additionally, some error estimates are available (see [42, Theorem 2.2]). All the results are gathered in the following theorem.

Theorem 5. (2nd order log-normal approximation with local volatility at spot). Assume $\left(\mathcal{H}_{x_{0}}^{a}\right)$. Assume that $h$ is locally Lipschitz in the following sense: for some constant $C_{h} \geq 0$,

$$
|h(x)| \leq C_{h} e^{C_{h}|x|}, \quad\left|\frac{h(y)-h(x)}{y-x}\right| \leq C_{h} e^{C_{h}(|x|+|y|)} \quad(\forall y \neq x) .
$$

Then

$$
\begin{aligned}
\mathbb{E}\left[h\left(X_{T}\right)\right]=\mathbb{E}\left[h\left(X_{T}^{P}\right)\right]+ & \left.C_{1}\left(a ; x_{0}\right)_{0}^{T}\left(\partial_{\varepsilon^{3}}^{3}-\frac{3}{2} \partial_{\varepsilon^{2}}^{2}+\frac{1}{2} \partial_{\varepsilon}\right) \mathbb{E}\left[h\left(X_{T}^{P}+\varepsilon\right)\right]\right|_{\varepsilon=0} \\
& +\mathcal{O}\left(\mathcal{M}_{1}(a)\left[\mathcal{M}_{0}(a)\right]^{2} T^{\frac{3}{2}}\right) .
\end{aligned}
$$

where the operator $C_{1}$ is defined in (35) and $\mathcal{O}$ depends notably of the constant $C_{h}$.

This formula is referred to as a second order approximation because the residual term is of order three with respect to the amplitude of the volatility coefficient.

Remark 6. The reader should notice that the expansion formulas are exact for the particular payoff function $h(x)=e^{x}$ (indeed $\mathbb{E}\left[h\left(X_{T}\right)\right]=\mathbb{E}\left[h\left(X_{T}^{P}\right)\right]=$ $\left.\partial_{\varepsilon^{i}}^{i} \mathbb{E}\left[h\left(X_{T}^{P}+\varepsilon\right)\right]\right|_{\varepsilon=0}=e^{x_{0}}$ and the sum of the corrective terms is equal to zero). This notably implies that the Call/Put parity relationship is preserved within these approximations, which is an essential property. The reader can verify in Section 5 that this martingale property is preserved for higher order approximation formulas.

Under the current assumptions $\left(\int_{0}^{T} a^{2}\left(t, x_{0}\right) \mathrm{d} t>0\right)$, the law of $X_{T}^{P}$ is a nondegenerate Gaussian r.v. and thus, the above derivatives are meaningful even for non-smooth $h$. Following [42], the Lipschitz regularity can be weakened to Holder regularity but error estimates in the case of discontinuous function $h$ are not available so far under the current set of assumptions.

$\triangleright$ Normal proxy. Alternatively to a log-normal proxy, we could prefer the use of normal proxy on the asset $S$ : for CEV-type model described in (27), it can be justified for $\beta$ close to 0 . The same analysis can be done by considering the normal proxy with diffusion coefficient computed at spot: it writes

$$
\mathrm{d} S_{t}^{P}=\Sigma\left(t, S_{0}\right) \mathrm{d} W_{t}, \quad S_{0}^{P}=S_{0} .
$$

Then, the distance to the proxy is represented through the interpolation process

$$
d S_{t}^{\eta}=\eta \Sigma\left(t, S_{t}^{\eta}\right) d W_{t}, \quad S_{0}^{\eta}=S_{0} .
$$


All the previous computations are very similar, and even simpler because there is no $\mathrm{d} t$-term. We skip details and state directly the result (see [42, Theorem 2.1]).

Theorem 7. (2nd order normal approximation with local volatility at spot). Assume $\left(\mathcal{H}_{S_{0}}^{\Sigma}\right)$. Assume that $h$ is locally Lipschitz in the following sense: for some constant $C_{h} \geq 0$,

$$
|h(x)| \leq C_{h}\left(1+|x|^{C_{h}}\right), \quad\left|\frac{h(y)-h(x)}{y-x}\right| \leq C_{h}\left(1+|x|^{C_{h}}+|y|^{C_{h}}\right) \quad(\forall y \neq x) .
$$

Then

$$
\mathbb{E}\left[h\left(S_{T}\right)\right]=\mathbb{E}\left[h\left(S_{T}^{P}\right)\right]+\left.C_{1}\left(\Sigma ; S_{0}\right)_{0}^{T} \partial_{\varepsilon^{3}}^{3} \mathbb{E}\left[h\left(S_{T}^{P}+\varepsilon\right)\right]\right|_{\varepsilon=0}+\mathcal{O}\left(\mathcal{M}_{1}(\Sigma)\left[\mathcal{M}_{0}(\Sigma)\right]^{2} T^{\frac{3}{2}}\right) .
$$

Remark 8. As for the log-normal proxy (see Remark 6), the approximation formulas involving the normal proxy does not suffer from numerical arbitrage when using Call/Put payoffs: indeed they are exact for the particular payoff function $h(x)=x$ (indeed $\mathbb{E}\left[h\left(S_{T}\right)\right]=\mathbb{E}\left[h\left(S_{T}^{P}\right)\right]=S_{0}$ and $\left.\left.\partial_{\varepsilon^{i}}^{i} \mathbb{E}\left[h\left(S_{T}^{P}+\varepsilon\right)\right]\right|_{\varepsilon=0}=0, \forall i \geq 2\right)$. This property holds again when considering higher order expansions (see Section 5 ).

Applying two previous results to the pricing of Call option (i.e. $h(x)=\left(e^{x}-K\right)_{+}$ in the case of log-normal proxy, and $h(x)=(x-K)+$ in the case of normal proxy), we obtain two different expansions using respectively Black-Scholes formula and Bachelier formula.

Theorem 9. (2nd order approximations for Call options with local volatility at spot). Assuming $\left(\mathcal{H}_{x_{0}}^{a}\right)$ and using the log-normal proxy, one has

$$
\begin{aligned}
\operatorname{Call}\left(e^{x_{0}}, T, e^{k}\right)= & \operatorname{Call}^{\mathrm{BS}}\left(x_{0}, \bar{a}_{x_{0}}^{2} T, k\right) \\
& +C_{1}\left(a ; x_{0}\right)_{0}^{T}\left(\partial_{x^{3}}^{3}-\frac{3}{2} \partial_{x^{2}}^{2}+\frac{1}{2} \partial_{x}\right) \operatorname{Call}^{\mathrm{BS}}\left(x_{0}, \bar{a}_{x_{0}}^{2} T, k\right) \\
& +\mathcal{O}\left(\mathcal{M}_{1}(a)\left[\mathcal{M}_{0}(a)\right]^{2} T^{\frac{3}{2}}\right) .
\end{aligned}
$$

Assuming $\left(\mathcal{H}_{S_{0}}^{\Sigma}\right)$ and using the normal proxy, one has

$$
\begin{aligned}
\operatorname{Call}\left(S_{0}, T, K\right)= & \operatorname{Call}^{\mathrm{BA}}\left(S_{0}, \bar{\Sigma}_{S_{0}}^{2} T, K\right)+C_{1}\left(\Sigma ; S_{0}\right)_{0}^{T} \partial_{S^{3}}^{3} \operatorname{Call}^{\mathrm{BA}}\left(S_{0}, \bar{\Sigma}_{S_{0}}^{2} T, K\right) \\
& +\mathcal{O}\left(\mathcal{M}_{1}(\Sigma)\left[\mathcal{M}_{0}(\Sigma)\right]^{2} T^{\frac{3}{2}}\right) .
\end{aligned}
$$

\subsection{Towards Call option approximations with the local volatility at strike and at mid-point}

For general payoff functions, the most natural choice seems to choose a proxy with the local volatility frozen at spot. When we are dealing with Call or Put payoffs, the spot and strike variables play a symmetrical role [43], and there is a priori no reason to advantage one or the other one. A first attempt to exploit this duality in proxy expansion is analysed in [44]. In this subsection, we briefly recall the expansion formulas with a local volatility at strike and then we present new expansion formulas 
with a local volatility at mid-point $x_{\mathrm{av}}=\left(x_{0}+k\right) / 2=\log \sqrt{S_{0} K}$ or $S_{\mathrm{av}}=\left(S_{0}+\right.$ $K) / 2$. We detail the analysis only for the log-normal proxy. The proofs for the normal proxy are very similar and are left as an exercise to the reader.

To directly obtain expansions formulas with local volatility frozen at strike, the idea is to follow the Dupire approach [43], using explicitly the PDE satisfied by the Call price function $(T, K) \rightarrow \operatorname{Call}\left(S_{0}, T, K\right)=\mathbb{E}\left[\left(S_{T}-K\right)_{+}\right]$. Indeed we have that:

$$
\left\{\begin{array}{l}
\partial_{T} \operatorname{Call}\left(S_{0}, T, K\right)=\frac{1}{2} \sigma^{2}(T, K) K^{2} \partial_{K^{2}}^{2} \operatorname{Call}\left(S_{0}, T, K\right), \\
\operatorname{Call}\left(S_{0}, 0, K\right)=\left(S_{0}-K\right)_{+} .
\end{array}\right.
$$

Thus we do not consider anymore a PDE in the backward variables $(t, S)$ with a Call payoff as a terminal condition, but we now handle a PDE in the forward variables $(T, K)$, with a put payoff condition. This dual PDE has a probabilistic Feynman-Kac representation:

$$
\operatorname{Call}\left(S_{0}, T, K\right)=\mathbb{E}\left[\left(S_{0}-e^{k_{T}}\right)_{+}\right],
$$

where $\left(k_{t}\right)_{t \in[0, T]}$ is the diffusion process defined by:

$$
\mathrm{d} k_{t}=a\left(T-t, k_{t}\right) \mathrm{d} W_{t}-\frac{1}{2} a^{2}\left(T-t, k_{t}\right) \mathrm{d} t, k_{0}=k=\log (K),
$$

where we recall that $a(t, z)=\sigma\left(t, e^{z}\right)$. Thus we are in a position to apply Theorem 5 for the Put payoff function $h(z)=\left(e^{x_{0}}-e^{z}\right)_{+}$with $\log$-strike $x_{0}=\log \left(S_{0}\right)$, with a log-normal proxy starting from $K=e^{k}$ and with the local volatility $\widetilde{a}(t, z)=$ $a(T-t, z)$. In the same way, we can apply Theorem 7 with a normal proxy. As a result, we obtain a variant of Theorem 9 where the Greeks w.r.t. the $k_{T}$-variable are naturally transformed into Greeks w.r.t. the strike variable. The final statement is the following result.

Theorem 10. (2nd order approximations for Call options with local volatility at strike). Assuming $\left(\mathcal{H}_{k}^{a}\right)$ and using the log-normal proxy, one has

$$
\begin{aligned}
\operatorname{Call}\left(e^{x_{0}}, T, e^{k}\right)= & \operatorname{Call}^{\mathrm{BS}}\left(x_{0}, \bar{a}_{k}^{2} T, k\right) \\
& +C_{1}(\widetilde{a} ; k)_{0}^{T}\left(\partial_{z^{3}}^{3}-\frac{3}{2} \partial_{z^{2}}^{2}+\frac{1}{2} \partial_{z}\right) \operatorname{Call}^{\mathrm{BS}}\left(x_{0}, \bar{a}_{k}^{2} T, k\right) \\
& +\mathcal{O}\left(\mathcal{M}_{1}(a)\left[\mathcal{M}_{0}(a)\right]^{2} T^{\frac{3}{2}}\right) .
\end{aligned}
$$

Assuming $\left(\mathcal{H}_{K}^{\Sigma}\right)$ and using the normal proxy, one has

$$
\begin{aligned}
\operatorname{Call}\left(S_{0}, T, K\right)= & \operatorname{Call}^{\mathrm{BA}}\left(S_{0}, \bar{\Sigma}_{K}^{2} T, K\right)+C_{1}(\widetilde{\Sigma} ; K)_{0}^{T} \partial_{Z^{3}}^{3} \operatorname{Call}^{\mathrm{BA}}\left(S_{0}, \bar{\Sigma}_{K}^{2} T, K\right) \\
& +\mathcal{O}\left(\mathcal{M}_{1}(\Sigma)\left[\mathcal{M}_{0}(\Sigma)\right]^{2} T^{\frac{3}{2}}\right) .
\end{aligned}
$$

Now, in order to obtain approximation formulas for the mid-points $x_{\mathrm{av}}$ or $S_{\mathrm{av}}$, we perform a Taylor expansion of the local volatility function around these midpoints. We start from the expansions at spot and strike given in Theorems 9 and 10, we consider the average of these expansions and we transform each term to freeze 
the local volatility function at $x_{\mathrm{av}}$ or $S_{\mathrm{av}}$. We only give details for the log-normal proxy. We first analyze the corrective terms.

Lemma 11. Assume $\left(\mathcal{H}_{x_{0}}^{a}\right)-\left(\mathcal{H}_{k}^{a}\right)-\left(\mathcal{H}_{x_{\text {av }}}^{a}\right)$. We have:

$$
\begin{aligned}
& \frac{1}{2} C_{1}\left(a ; x_{0}\right)_{0}^{T}\left(\partial_{x^{3}}^{3}-\frac{3}{2} \partial_{x^{2}}^{2}+\frac{1}{2} \partial_{x}\right) \mathrm{Call}^{\mathrm{BS}}\left(x_{0}, \bar{a}_{x_{0}}^{2} T, k\right) \\
& +\frac{1}{2} C_{1}(\widetilde{a} ; k)_{0}^{T}\left(\partial_{z^{3}}^{3}-\frac{3}{2} \partial_{z^{2}}^{2}+\frac{1}{2} \partial_{z}\right) \mathrm{Call}^{\mathrm{BS}}\left(x_{0}, \bar{a}_{k}^{2} T, k\right) \\
= & \frac{1}{2}\left[C_{1}\left(a ; x_{\mathrm{av}}\right)_{0}^{T}-C_{1}\left(\widetilde{a} ; x_{\mathrm{av}}\right)_{0}^{T}\right]\left(\partial_{x^{3}}^{3}-\frac{3}{2} \partial_{x^{2}}^{2}+\frac{1}{2} \partial_{x}\right) \mathrm{Call}^{\mathrm{BS}}\left(x_{0}, \bar{a}_{x_{\mathrm{av}}}^{2} T, k\right) \\
& +\mathcal{O}\left(\mathcal{M}_{1}(a)\left[\mathcal{M}_{0}(a)\right]^{2} T^{\frac{3}{2}}\right) .
\end{aligned}
$$

Proof. We begin with the $x_{0}$-Greeks. Perform a zero order Taylor formula for the function $y \rightarrow\left(\partial_{x^{3}}^{3}-\frac{3}{2} \partial_{x^{2}}^{2}+\frac{1}{2} \partial_{x}\right) \mathrm{Call}^{\mathrm{BS}}\left(x_{0}, y, k\right)$ at $y=\bar{a}_{x_{0}}^{2} T=\omega\left(a^{2}\left(x_{0}\right)\right)_{0}^{T}$ around $y=\bar{a}_{x_{\mathrm{av}}}^{2} T=\omega\left(a^{2}\left(x_{\mathrm{av}}\right)\right)_{0}^{T}$ and $\forall t \in[0, T]$, for the function $x \rightarrow a_{t}^{2}(x)$ at $x=x_{0}$ around $x=x_{\text {av }}$ to obtain:

$$
\begin{aligned}
& C_{1}\left(a ; x_{0}\right)_{0}^{T}\left(\partial_{x^{3}}^{3}-\frac{3}{2} \partial_{x^{2}}^{2}+\frac{1}{2} \partial_{x}\right) \operatorname{Call}^{\mathrm{BS}}\left(x_{0}, \bar{a}_{x_{0}}^{2} T, k\right) \\
= & {\left[C_{1}\left(a ; x_{\mathrm{av}}\right)_{0}^{T}+R_{1}\right]\left[\left(\partial_{x^{3}}^{3}-\frac{3}{2} \partial_{x^{2}}^{2}+\frac{1}{2} \partial_{x}\right) \mathrm{Call}^{\mathrm{BS}}\left(x_{0}, \bar{a}_{x_{\mathrm{av}}}^{2} T, k\right)+R_{2}\right] } \\
= & C_{1}\left(a ; x_{\mathrm{av}}\right)_{0}^{T}\left(\partial_{x^{3}}^{3}-\frac{3}{2} \partial_{x^{2}}^{2}+\frac{1}{2} \partial_{x}\right) \mathrm{Call}^{\mathrm{BS}}\left(x_{0}, \bar{a}_{x_{\mathrm{av}}}^{2} T, k\right) \\
& +\left(\partial_{x^{3}}^{3}-\frac{3}{2} \partial_{x^{2}}^{2}+\frac{1}{2} \partial_{x}\right) \mathrm{Call}^{\mathrm{BS}}\left(x_{0}, \bar{a}_{x_{\mathrm{av}}}^{2} T, k\right) R_{1}+C_{1}\left(a ; x_{0}\right)_{0}^{T} R_{2},
\end{aligned}
$$

where:

$$
\begin{aligned}
R_{1}= & \left.\frac{\left(x_{0}-k\right)}{2} \int_{0}^{1}\left(\partial_{x} C_{1}(a ; x)\right)_{0}^{T}\right)\left.\right|_{x=\lambda x_{0}+(1-\lambda) x_{\mathrm{av}}} \mathrm{d} \lambda \\
R_{2}= & T\left(\bar{a}_{x_{0}}^{2}-\bar{a}_{x_{\mathrm{av}}}^{2}\right) \\
& \times\left.\int_{0}^{1}\left(\partial_{y x^{3}}^{4}-\frac{3}{2} \partial_{y x^{2}}^{3}+\frac{1}{2} \partial_{y x}^{2}\right) \mathrm{Call}^{\mathrm{BS}}\left(x_{0}, y, k\right)\right|_{y=T\left(\lambda \bar{a}_{x_{0}}^{2}+(1-\lambda) \bar{a}_{x_{\mathrm{av}}}^{2}\right)} \mathrm{d} \lambda, \\
T\left(\bar{a}_{x_{0}}^{2}-\bar{a}_{x_{\mathrm{av}}}^{2}\right)= & \left.\frac{\left(x_{0}-k\right)}{2} \int_{0}^{1}\left(\partial_{x} \omega\left(a^{2}(x)\right)_{0}^{T}\right)\right|_{x=\lambda x_{0}+(1-\lambda) x_{\mathrm{av}}} \mathrm{d} \lambda .
\end{aligned}
$$

In view of the definition (35) of $C_{1}$, the identity (6), Corollary 30 and $\left(\mathcal{H}_{x_{0}}^{a}\right)-\left(\mathcal{H}_{x_{\text {av }}}^{a}\right)$, we readily obtain

$$
\begin{aligned}
& \mid\left(\partial_{x^{3}}^{3}-\frac{3}{2} \partial_{x^{2}}^{2}+\right.\left.\frac{1}{2} \partial_{x}\right) \operatorname{Call}^{\mathrm{BS}}\left(x_{0}, \bar{a}_{x_{\mathrm{av}}}^{2} T, k\right) R_{1} \mid \\
& \leq \frac{1}{2} \mid\left(\partial_{x^{3}}^{3}-\frac{3}{2} \partial_{x^{2}}^{2}+\frac{1}{2} \partial_{x}\right) \operatorname{Call}^{\mathrm{BS}}\left(x_{0}, \bar{a}_{x_{\mathrm{av}}}^{2} T, k\right)\left(x_{0}-k\right) \mid \\
& \quad \times\left|\int_{0}^{1}\left(\partial_{x} C_{1}(a ; x)_{0}^{T}\right)\right|_{x=\lambda x_{0}+(1-\lambda) x_{\mathrm{av}}} \mathrm{d} \lambda \mid \\
& \leq \leq_{c}\left[\bar{a}_{x_{\mathrm{av}}}^{2} T\right]^{-\frac{1}{2}} \mathcal{M}_{1}(a)\left[\mathcal{M}_{0}(a)\right]^{3} T^{2} \leq_{c} \mathcal{M}_{1}(a)\left[\mathcal{M}_{0}(a)\right]^{2} T^{\frac{3}{2}}
\end{aligned}
$$




$$
\begin{aligned}
& \left|C_{1}\left(a ; x_{0}\right)_{0}^{T} R_{2}\right| \\
& \quad \leq_{c}\left[\mathcal{M}_{0}(a)\right]^{3} \mathcal{M}_{1}(a) T^{2} \mathcal{M}_{0}(a) \mathcal{M}_{1}(a) T \int_{0}^{1}\left[T\left(\lambda \bar{a}_{x_{0}}^{2}+(1-\lambda) \bar{a}_{x_{\mathrm{av}}}^{2}\right)\right]^{-\frac{3}{2}} \mathrm{~d} \lambda \\
& \quad \leq_{c} \mathcal{M}_{1}(a)\left[\mathcal{M}_{0}(a)\right]^{2} T^{\frac{3}{2}} .
\end{aligned}
$$

Similarly, using in addition $\left(\mathcal{H}_{k}^{a}\right)$ we show that:

$$
\begin{aligned}
& C_{1}(\widetilde{a} ; k)_{0}^{T}\left(\partial_{z^{3}}^{3}-\frac{3}{2} \partial_{z^{2}}^{2}+\frac{1}{2} \partial_{z}\right) \operatorname{Call}^{\mathrm{BS}}\left(x_{0}, \bar{a}_{k}^{2} T, k\right) \\
= & C_{1}\left(\widetilde{a} ; x_{\mathrm{av}}\right)_{0}^{T}\left(\partial_{z^{3}}^{3}-\frac{3}{2} \partial_{z^{2}}^{2}+\frac{1}{2} \partial_{z}\right) \mathrm{Call}^{\mathrm{BS}}\left(x_{0}, \bar{a}_{x_{\mathrm{av}}}^{2} T, k\right)+\mathcal{O}\left(\mathcal{M}_{1}(a)\left[\mathcal{M}_{0}(a)\right]^{2} T^{\frac{3}{2}}\right), \\
= & -C_{1}\left(\widetilde{a} ; x_{\mathrm{av}}\right)_{0}^{T}\left(\partial_{x^{3}}^{3}-\frac{3}{2} \partial_{x^{2}}^{2}+\frac{1}{2} \partial_{x}\right) \operatorname{Call}^{\mathrm{BS}}\left(x_{0}, \bar{a}_{x_{\mathrm{av}}}^{2} T, k\right)+\mathcal{O}\left(\mathcal{M}_{1}(a)\left[\mathcal{M}_{0}(a)\right]^{2} T^{\frac{3}{2}}\right),
\end{aligned}
$$

where we have used at the last equality the relation (72) in Proposition 34. That completes the proof.

Second, we analyze the leading order of the formula given in Theorems 9 and 10:

Lemma 12. Assume $\left(\mathcal{H}_{x_{0}}^{a}\right)-\left(\mathcal{H}_{k}^{a}\right)-\left(\mathcal{H}_{x_{\mathrm{av}}}^{a}\right)$. We have:

$$
\begin{aligned}
\frac{1}{2}\left[\operatorname{Call}^{\mathrm{BS}}\left(x_{0}, \bar{a}_{x_{0}}^{2} T, k\right)+\operatorname{Call}^{\mathrm{BS}}\left(x_{0}, \bar{a}_{k}^{2} T, k\right)\right]= & \mathrm{Call}^{\mathrm{BS}}\left(x_{0}, \bar{a}_{x_{\mathrm{av}}}^{2} T, k\right) \\
& +\mathcal{O}\left(\mathcal{M}_{1}(a)\left[\mathcal{M}_{0}(a)\right]^{2} T^{\frac{3}{2}}\right) .
\end{aligned}
$$

Proof. Apply a first order Taylor formula twice; firstly for the function $y \rightarrow$ Call ${ }^{\mathrm{BS}}\left(x_{0}, y, k\right)$ at $y=\bar{a}_{x_{0}}^{2} T$ around $y=\bar{a}_{x_{\mathrm{av}}}^{2} T$ and secondly, for the function $x \rightarrow a_{t}^{2}(x)$ at $x=x_{0}$ around $x=x_{\mathrm{av}}, \forall t \in[0, T]$. It gives

$$
\begin{aligned}
& \operatorname{Call}^{\mathrm{BS}}\left(x_{0}, \bar{a}_{x_{0}}^{2} T, k\right) \\
= & \operatorname{Call}^{\mathrm{BS}}\left(x_{0}, \bar{a}_{x_{\mathrm{av}}}^{2} T, k\right)+\partial_{y} \operatorname{Call}^{\mathrm{BS}}\left(x_{0}, \bar{a}_{x_{\mathrm{av}}}^{2} T, k\right) T\left(\bar{a}_{x_{0}}^{2}-\bar{a}_{x_{\mathrm{av}}}^{2}\right)+R_{1}, \\
= & \mathrm{Call}^{\mathrm{BS}}\left(x_{0}, \bar{a}_{x_{\mathrm{av}}}^{2} T, k\right)+\partial_{y} \operatorname{Call}^{\mathrm{BS}}\left(x_{0}, \bar{a}_{x_{\mathrm{av}}}^{2} T, k\right) \omega\left(a\left(x_{\mathrm{av}}\right) a^{(1)}\left(x_{\mathrm{av}}\right)\right)_{0}^{T}\left(x_{0}-k\right)+R_{2} \\
& +R_{1} .
\end{aligned}
$$

where:

$$
\begin{aligned}
& R_{1}=\left.T^{2}\left(\bar{a}_{x_{0}}^{2}-\bar{a}_{x_{\mathrm{av}}}^{2}\right)^{2} \int_{0}^{1}\left(\partial_{y^{2}}^{2} \operatorname{Call}^{\mathrm{BS}}\left(x_{0}, y, k\right)\right)\right|_{y=T\left(\lambda \bar{a}_{x_{0}}^{2}+(1-\lambda) \bar{a}_{x_{\mathrm{av}}}^{2}\right.}(1-\lambda) \mathrm{d} \lambda, \\
& R_{2}=\left.\partial_{y} \operatorname{Call}^{\mathrm{BS}}\left(x_{0}, \bar{a}_{x_{\mathrm{av}}}^{2} T, k\right) \frac{\left(x_{0}-k\right)^{2}}{4} \int_{0}^{1}\left(\partial_{x^{2}}^{2} \omega\left(a^{2}(x)\right)_{0}^{T}\right)\right|_{x=\lambda x_{0}+(1-\lambda) x_{\mathrm{av}}}(1-\lambda) \mathrm{d} \lambda .
\end{aligned}
$$

Similar arguments previously employed in the proof of Lemma 11 easily lead to:

$$
\begin{aligned}
\left|R_{1}\right| & \leq_{c}\left[\mathcal{M}_{1}(a)\right]^{2}\left[\mathcal{M}_{0}(a)\right]^{2} T^{2} \int_{0}^{1}\left[T\left(\lambda \bar{a}_{x_{0}}^{2}+(1-\lambda) \bar{a}_{x_{\mathrm{av}}}^{2}\right)\right]^{-\frac{1}{2}} \mathrm{~d} \lambda \\
& \leq_{c} \mathcal{M}_{1}(a)\left[\mathcal{M}_{0}(a)\right]^{2} T^{\frac{3}{2}} \\
\left|R_{2}\right| & \leq_{c} \mathcal{M}_{1}(a)\left[\mathcal{M}_{0}(a)\right]^{2} T^{\frac{3}{2}} .
\end{aligned}
$$


Similarly we have:

$$
\begin{aligned}
& \text { Call }^{\mathrm{BS}}\left(x_{0}, \bar{a}_{k}^{2} T, k\right) \\
= & \text { Call }^{\mathrm{BS}}\left(x_{0}, \bar{a}_{x_{\mathrm{av}}}^{2} T, k\right)-\partial_{y} \operatorname{Call}^{\mathrm{BS}}\left(x_{0}, \bar{a}_{x_{\mathrm{av}}}^{2} T, k\right) \omega\left(a\left(x_{\mathrm{av}}\right) a^{(1)}\left(x_{\mathrm{av}}\right)\right)_{0}^{T}\left(x_{0}-k\right) \\
& +\mathcal{O}\left(\mathcal{M}_{1}(a)\left[\mathcal{M}_{0}(a)\right]^{2} T^{\frac{3}{2}}\right) .
\end{aligned}
$$

We are finished.

Lemmas 11 and 12 lead to the following Theorem for the log-normal proxy, while similar arguments apply for the normal proxy.

Theorem 13. (2nd order approximations for Call options with local volatility at mid-point). Under $\left(\mathcal{H}_{x_{0}}^{a}\right)-\left(\mathcal{H}_{k}^{a}\right)-\left(\mathcal{H}_{x_{\mathrm{av}}}^{a}\right)$, we have

$$
\begin{aligned}
& \operatorname{Call}\left(e^{x_{0}}, T, e^{k}\right)=\operatorname{Call}^{\mathrm{BS}}\left(x_{0}, \bar{a}_{x_{\mathrm{av}}}^{2} T, k\right) \\
& \quad+\frac{C_{1}\left(a ; x_{\mathrm{av}}\right)_{0}^{T}-C_{1}\left(\widetilde{a} ; x_{\mathrm{av}}\right)_{0}^{T}}{2}\left(\partial_{x^{3}}^{3}-\frac{3}{2} \partial_{x^{2}}^{2}+\frac{1}{2} \partial_{x}\right) \operatorname{Call}^{\mathrm{BS}}\left(x_{0}, \bar{a}_{x_{\mathrm{av}}}^{2} T, k\right) \\
& \quad+\mathcal{O}\left(\mathcal{M}_{1}(a)\left[\mathcal{M}_{0}(a)\right]^{2} T^{\frac{3}{2}}\right), \\
& \begin{aligned}
\operatorname{Under}\left(\mathcal{H}_{S_{0}}^{\Sigma}\right)-\left(\mathcal{H}_{K}^{\Sigma}\right)-\left(\mathcal{H}_{S_{\mathrm{av}}}^{\Sigma}\right), \text { we have } \\
\operatorname{Call}\left(S_{0}, T, K\right)= \\
\quad \operatorname{Call}^{\mathrm{BA}}\left(S_{0}, \bar{\Sigma}_{S_{\mathrm{av}}}^{2} T, K\right) \\
\quad+\frac{C_{1}\left(\Sigma ; S_{\mathrm{av}}\right)_{0}^{T}-C_{1}\left(\widetilde{\Sigma} ; S_{\mathrm{av}}\right)_{0}^{T}}{2} \partial_{S^{3}}^{3} \operatorname{Call}^{\mathrm{BA}}\left(S_{0}, \bar{\Sigma}_{S_{\mathrm{av}}}^{2} T, K\right) \\
\quad+\mathcal{O}\left(\mathcal{M}_{1}(\Sigma)\left[\mathcal{M}_{0}(\Sigma)\right]^{2} T^{\frac{3}{2}}\right) .
\end{aligned}
\end{aligned}
$$

Remark 14. If $a$ (and consequently $\Sigma$ ) is time-independent or has separable variables, observe that the corrective terms vanish and we obtain remarkably simple formulas: the expansion formulas (37) and (38) reduce to only a Black-Scholes price and a Bachelier price, with the local volatility function frozen at the mid-point.

\subsection{Second order expansion of the implied volatility}

Interestingly, the previous expansions of Call price (Theorems 9, 10 and 13) can be turned into expansions of Black-Scholes and Bachelier implied volatility defined respectively in (7) and (9). To achieve this, we use the relations between Greeks postponed in Propositions 34 and 39 in order to write the different approximation formulas in terms of the Vega. For example consider the second order log-normal expansion formula based on the ATM local volatility (Theorem 9): thanks to (72) in Proposition 34, it becomes:

$$
\begin{aligned}
\operatorname{Call}\left(e^{x_{0}}, T, e^{k}\right)= & \operatorname{Call}^{\mathrm{BS}}\left(x_{0}, \bar{a}_{x_{0}}^{2} T, k\right)-\operatorname{Vega}^{\mathrm{BS}}\left(x_{0}, \bar{a}_{x_{0}}^{2} T, k\right) \frac{C_{1}\left(a ; x_{0}\right)_{0}^{T} m}{\bar{a}_{x_{0}}^{3} T^{2}} \\
& +\mathcal{O}\left(\mathcal{M}_{1}(a)\left[\mathcal{M}_{0}(a)\right]^{2} T^{\frac{3}{2}}\right), \\
\approx & \operatorname{Call}^{\mathrm{BS}}\left(x_{0},\left(\bar{a}_{x_{0}}-\frac{C_{1}\left(a ; x_{0}\right)_{0}^{T}}{\bar{a}_{x_{0}}^{3} T^{2}} m\right)^{2} T, k\right),
\end{aligned}
$$


where $m$ is the $\log$-moneyness $m=x_{0}-k=\log \left(S_{0} / K\right)$. We have paved the way for the following result:

Theorem 15. (2nd order expansions of the implied volatility). Assuming $\left(\mathcal{H}_{x_{0}}^{a}\right)-\left(\mathcal{H}_{k}^{a}\right)-\left(\mathcal{H}_{x_{\mathrm{av}}}^{a}\right)$ and using the log-normal proxy, we have

$$
\begin{aligned}
& \sigma_{\mathrm{I}}\left(x_{0}, T, k\right)=\bar{a}_{x_{0}}-\frac{C_{1}\left(a ; x_{0}\right)_{0}^{T}}{\bar{a}_{x_{0}}^{3} T^{2}} m+\operatorname{Error}_{2, \mathrm{x}_{0}}^{\mathrm{I}}, \\
& \sigma_{\mathrm{I}}\left(x_{0}, T, k\right)=\bar{a}_{k}+\frac{C_{1}(\widetilde{a} ; k)_{0}^{T}}{\bar{a}_{k}^{3} T^{2}} m+\operatorname{Error}_{2, \mathrm{k}}^{\mathrm{I}}, \\
& \sigma_{\mathrm{I}}\left(x_{0}, T, k\right)=\bar{a}_{x_{\mathrm{av}}}+\frac{\left(C_{1}\left(\widetilde{a} ; x_{\mathrm{av}}\right)_{0}^{T}-C_{1}\left(a ; x_{\mathrm{av}}\right)_{0}^{T}\right)}{2 \bar{a}_{x_{\mathrm{av}}}^{3} T^{2}} m+\text { Error }_{2, \mathrm{x}_{\mathrm{av}}}^{\mathrm{I}} .
\end{aligned}
$$

Assuming $\left(\mathcal{H}_{S_{0}}^{\Sigma}\right)-\left(\mathcal{H}_{K}^{\Sigma}\right)-\left(\mathcal{H}_{S_{\mathrm{av}}}^{\Sigma}\right)$ and using the normal proxy, we have

$$
\begin{aligned}
& \Sigma_{\mathrm{I}}\left(S_{0}, T, K\right)=\bar{\Sigma}_{S_{0}}-\frac{C_{1}\left(\Sigma ; S_{0}\right)_{0}^{T}}{\bar{\Sigma}_{S_{0}}^{3} T^{2}} M+\operatorname{Error}_{2, \mathrm{~S}_{0}}^{\mathrm{I}}, \\
& \Sigma_{\mathrm{I}}\left(S_{0}, T, K\right)=\bar{\Sigma}_{K}+\frac{C_{1}(\widetilde{\Sigma} ; K)_{0}^{T}}{\bar{\Sigma}_{K}^{3} T^{2}} M+\operatorname{Error}_{2, \mathrm{~K}}^{\mathrm{I}}, \\
& \Sigma_{\mathrm{I}}\left(S_{0}, T, K\right)=\bar{\Sigma}_{S_{\mathrm{av}}}+\frac{\left(C_{1}\left(\widetilde{\Sigma} ; S_{\mathrm{av}}\right)_{0}^{T}-C_{1}\left(\Sigma ; S_{\mathrm{av}}\right)_{0}^{T}\right)}{2 \bar{\Sigma}_{S_{\mathrm{av}}^{3}}^{3} T^{2}} M+\operatorname{Error}_{2, \mathrm{~S}_{\mathrm{av}}}^{\mathrm{I}},
\end{aligned}
$$

where $S_{\mathrm{av}}=\frac{S_{0}+K}{2}$ and $M=S_{0}-K$.

Remark 16. We retrieve in our implied volatility approximation formulas the wellknown property that at the money (ie $m=0$ ) and for short maturity, the value of the implied volatility is equal to the value of the local volatility function and the slope of the local volatility function is twice the slope of the implied volatility. We justify this assertion for the Black-Scholes implied volatility, the work being similar for the Bachelier one. If $T \ll 1$, in view of (39) and the definition (35) of $C_{1}$, assuming that $a\left(t, x_{0}\right)$ and $a^{(1)}\left(t, x_{0}\right)$ are continuous at $t=0$, we obtain:

$$
\begin{aligned}
{\left.\left[\sigma_{\mathrm{I}}\left(x_{0}, T, k\right)\right]\right|_{k=x_{0}} } & \approx a\left(0, x_{0}\right), \\
\left.\partial_{k}\left[\sigma_{\mathrm{I}}\left(x_{0}, T, k\right)\right]\right|_{k=x_{0}} & \left.\approx \partial_{k}\left[\bar{a}_{x_{0}}\right]\right|_{k=x_{0}}-\left.\frac{C_{1}\left(a ; x_{0}\right)_{0}^{T}}{\bar{a}_{x_{0}}^{3} T^{2}} \partial_{k}\left[\left(x_{0}-k\right)\right]\right|_{k=x_{0}} \\
& \approx 0+\frac{a^{3}\left(0, x_{0}\right) a^{(1)}\left(0, x_{0}\right) \frac{T^{2}}{2}}{a^{3}\left(0, x_{0}\right) T^{2}}=\frac{a^{(1)}\left(0, x_{0}\right)}{2} .
\end{aligned}
$$

We obtain the same estimates starting from (40) and (41), we skip details.

To conclude this paragraph, we estimate the residual terms of the above implied volatility expansions, in terms of $\mathcal{M}_{0}(a), \mathcal{M}_{1}(a)$ and so on. Since the Vega is very small for far OTM/ITM Call options, deriving error bounds on implied volatility from Theorems 9, 10 and 13 gives poor estimates for extreme strikes. Actually, in the further numerical experiments, we also observe inaccuracies for extreme strikes. 
To obtain accurate theoretical error bounds, we restrict to log-moneyness $m$ (resp moneyness $M$ ) belonging to a small ball by assuming that $|m| \leq \xi \mathcal{M}_{0}(a) \sqrt{T}$ (resp. $\left.|M| \leq \xi \mathcal{M}_{0}(\Sigma) \sqrt{T}\right)$ for a given $\xi>0$.

For the sake of brevity, we only analyze the expansion (39), the other approximations being similar. We assume in addition that $\mathcal{M}_{0}(a), \mathcal{M}_{1}(a)$ and $T$ are globally small enough to ensure that $\bar{a}_{x_{0}}-\frac{C_{1}\left(a ; x_{0}\right)_{0}^{T}}{\bar{a}_{x_{0}}^{3} T^{2}} m>0$. Note that at the money (i.e. $m=0$ ), this condition is automatically satisfied. A first order expansion readily gives

$$
\begin{aligned}
& \operatorname{Call}^{\mathrm{BS}}\left(x_{0},\left(\bar{a}_{x_{0}}-\frac{C_{1}\left(a ; x_{0}\right)_{0}^{T}}{\bar{a}_{x_{0}}^{3} T^{2}} m\right)^{2} T, k\right) \\
= & \operatorname{Call}^{\mathrm{BS}}\left(x_{0}, \bar{a}_{x_{0}}^{2} T, k\right)-\frac{C_{1}\left(a ; x_{0}\right)_{0}^{T}}{\bar{a}_{x_{0}}^{3} T^{2}} m \operatorname{Vega}^{\mathrm{BS}}\left(x_{0}, \bar{a}_{x_{0}}^{2} T, k\right) \\
& +\left.\left(\frac{C_{1}\left(a ; x_{0}\right)_{0}^{T}}{\bar{a}_{x_{0}}^{3} T^{2}} m\right)^{2} \int_{0}^{1} \operatorname{Vomma}^{\mathrm{BS}}\left(x_{0}, a^{2} T, k\right)\right|_{a=\bar{a}_{x_{0}}-\lambda \frac{C_{1}\left(a ; x_{0}\right)_{0}^{T}}{\bar{a}_{x_{0}}^{3} T^{2}} m}(1-\lambda) \mathrm{d} \lambda \\
= & \operatorname{Call}^{\mathrm{BS}}\left(x_{0}, \sigma_{\mathrm{I}}^{2}\left(x_{0}, T, k\right) T, k\right)+\mathcal{O}\left(\mathcal{M}_{1}(a)\left[\mathcal{M}_{0}(a)\right]^{2} T^{\frac{3}{2}}\right) \\
& +\left.\left(\frac{C_{1}\left(a ; x_{0}\right)_{0}^{T}}{\bar{a}_{x_{0}}^{3} T^{2}} m\right)^{2} \int_{0}^{1} \operatorname{Vomma}^{\mathrm{BS}}\left(x_{0}, a^{2} T, k\right)\right|_{a=\bar{a}_{x_{0}}-\lambda \frac{C_{1}\left(a ; x_{0}\right)_{0}^{T}}{\bar{a}_{x_{0}}^{3} T^{2}} m}(1-\lambda) \mathrm{d} \lambda,
\end{aligned}
$$

applying Theorem 9 and using the definition of the Black-Scholes implied volatility. Expanding $a \rightarrow \mathrm{Call}^{\mathrm{BS}}\left(x_{0}, a^{2} T, k\right)$ at $a=\sigma_{\mathrm{I}}\left(x_{0}, T, k\right)$ around $a=\bar{a}_{x_{0}}-\frac{C_{1}\left(a ; x_{0}\right)_{0}^{T}}{\bar{a}_{x_{0}}^{3} T^{2}} m$ gives:

$$
\begin{aligned}
\operatorname{Error}_{2, x_{0}}^{\mathrm{I}} & \left.\int_{0}^{1} \operatorname{Vega}^{\mathrm{BS}}\left(x_{0}, a^{2} T, k\right)\right|_{a=\sigma_{\mathrm{I}}\left(x_{0}, T, k\right)-\lambda \operatorname{Error}_{2, x_{0}}^{\mathrm{I}}} \mathrm{d} \lambda=\mathcal{O}\left(\mathcal{M}_{1}(a)\left[\mathcal{M}_{0}(a)\right]^{2} T^{\frac{3}{2}}\right) \\
& -\left.\left(\frac{C_{1}\left(a ; x_{0}\right)_{0}^{T}}{\bar{a}_{x_{0}}^{3} T^{2}} m\right)^{2} \int_{0}^{1} \operatorname{Vomma}^{\mathrm{BS}}\left(x_{0}, a^{2} T, k\right)\right|_{a=\bar{a}_{x_{0}}-\lambda \frac{C_{1}\left(a ; x_{0}\right)_{0}^{T}}{\bar{a}_{x_{0}}^{3} T^{2}} m}(1-\lambda) \mathrm{d} \lambda .
\end{aligned}
$$

In view of the expression of Vega ${ }^{\mathrm{BS}}$ (see (65) in Proposition 32) and (68) in Corollary 33 , the hypotheses made on $m, \mathcal{M}_{0}(a), \mathcal{M}_{1}(a)$ and $T$ guarantee the existence of a constant $C>0$ (depending on $S_{0}$ ) such that:

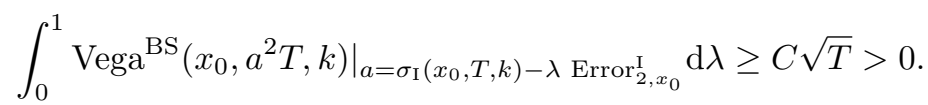

In addition $(69)$ and $\left(\mathcal{H}_{x_{0}}^{a}\right)$ readily yield

$$
\begin{aligned}
& \left|\left(\frac{C_{1}\left(a ; x_{0}\right)_{0}^{T}}{\bar{a}_{x_{0}}^{3} T^{2}} m\right)^{2} \int_{0}^{1} \operatorname{Vomma}^{\mathrm{BS}}\left(x_{0}, a^{2} T, k\right)\right|_{a=\bar{a}_{x_{0}}-\lambda \frac{C_{1}\left(a ; x_{0}\right)_{0}^{T}}{\bar{a}_{x_{0}}^{3} T^{2}} m}(1-\lambda) \mathrm{d} \lambda \mid \\
\leq_{c}\left(\mathcal{M}_{1}(a) \mathcal{M}_{0}(a) \sqrt{T}\right)^{2} & \frac{\sqrt{T}}{\bar{a}_{x_{0}}} \leq c \mathcal{M}_{1}(a)\left[\mathcal{M}_{0}(a)\right]^{2} T^{\frac{3}{2}},
\end{aligned}
$$

where the generic constant depends in an increasing way on $\xi$. That finally implies:

$$
\operatorname{Error}_{2, x_{0}}^{\mathrm{I}}=\mathcal{O}\left(\mathcal{M}_{1}(a)\left[\mathcal{M}_{0}(a)\right]^{2} T\right) \text {. }
$$


In view of the above upper bound, we interpret our implied volatility formulas as second order expansion ones.

\section{Proofs: a comparative discussion between stochastic analysis and PDE techniques}

In this section, our aim is to show how three different techniques ranging from stochastic analysis to PDE may lead to the same formulas given in Theorem 5. Although the final result is the same, the derivation is quite different, first regarding the way in which the expansion coefficients are made explicit, second regarding the error estimates and the assumptions used for that.

We shall admit that our preference is for the stochastic analysis approach, because it is flexible regarding the model and the functionals under consideration, and it is slightly less demanding regarding the assumptions (pointwise ellipticity versus uniform ellipticity for instance). But the reader may argue differently, depending on its own fields of expertise.

As an illustration of flexibility of the stochastic analysis approach, it has been possible to handle Call/Put/digital options in local volatility models with Gaussian jumps [45], Call/Put options in local volatility models with stochastic Gaussian interest rates [7], Call/Put options in time-dependent Heston model [46], general average options (including Asian and Basket options) in local volatility models [42], and more recently local stochastic volatility models [47].

\subsection{A pure stochastic analysis approach}

This is basically the derivation that we have performed in Subsection 3.2.

Smooth payoff $\boldsymbol{h}$. We first deal with the case of infinitely differentiable function $h$ with exponentially bounded derivatives. Resuming from (31-32-33) and using Taylor's formula, write

$$
\begin{aligned}
\mathbb{E}\left[h\left(X_{T}\right)\right]= & \mathbb{E}\left[h\left(X_{T}^{P}\right)\right]+\mathbb{E}\left[h^{(1)}\left(X_{T}^{P}\right)\left(X_{T}-X_{T}^{P}\right)\right] \\
& +\int_{0}^{1} \mathbb{E}\left[h^{(2)}\left(X_{T}^{P}+\lambda\left(X_{T}-X_{T}^{P}\right)\right)\left(X_{T}-X_{T}^{P}\right)^{2}\right](1-\lambda) \mathrm{d} \lambda \\
= & \mathbb{E}\left[h\left(X_{T}^{P}\right)\right]+\mathbb{E}\left[h^{(1)}\left(X_{T}^{P}\right) \frac{X_{2, T}}{2}\right]+\mathbb{E}\left[h^{(1)}\left(X_{T}^{P}\right) \int_{0}^{1} \frac{(1-\lambda)^{2}}{2} X_{3, T}^{\lambda}\right] \mathrm{d} \lambda \\
& +\int_{0}^{1} \mathbb{E}\left[h^{(2)}\left(X_{T}^{P}+\lambda\left(X_{T}-X_{T}^{P}\right)\right)\left(\int_{0}^{1} X_{2, T}^{\eta}(1-\eta) \mathrm{d} \eta\right)^{2}\right](1-\lambda) \mathrm{d} \lambda \\
:= & \mathbb{E}\left[h\left(X_{T}^{P}\right)\right]+\mathbb{E}\left[h^{(1)}\left(X_{T}^{P}\right) \frac{X_{2, T}}{2}\right]+\operatorname{Error}_{2}(h) .
\end{aligned}
$$

The first correction term $\mathbb{E}\left[h^{(1)}\left(X_{T}^{P}\right) \frac{X_{2, T}}{2}\right]$ is made explicit using the key Lemma 4 , and it is equal to a weighted summation of sensitivities $\left.\partial_{\varepsilon}^{i} \mathbb{E}\left[h\left(X_{T}^{P}+\varepsilon\right)\right]\right|_{\varepsilon=0}$ for 
$i=1,2,3$ (see the statement of Theorem 5 ).

The evaluation of $\operatorname{Error}_{2}(h)$ requires to estimate the $L_{p}$-norms of $X_{2, T}^{\lambda}$ and $X_{3, T}^{\lambda}$ (uniformly in $\lambda \in[0,1]$ ). Direct and standard stochastic calculus inequalities from (28-29-30) yield

$$
\left|X_{2, T}^{\lambda}\right|_{p} \leq_{c} \mathcal{M}_{1}(a) \mathcal{M}_{0}(a) T, \quad\left|X_{3, T}^{\lambda}\right|_{p} \leq_{c} \mathcal{M}_{1}(a)\left[\mathcal{M}_{0}(a)\right]^{2} T^{3 / 2}
$$

for any $p \geq 1$ and any $\lambda \in[0,1]$. Combining these estimates with Hölder and Minkowski inequalities readily gives $\operatorname{Error}_{2}(h)=\mathcal{O}\left(\mathcal{M}_{1}(a)\left[\mathcal{M}_{0}(a)\right]^{2} T^{\frac{3}{2}}\right)$, which completes the proof if $h$ is smooth as above. Observe that we have only required the coefficients to be smooth enough, and nothing has been imposed on the nondegeneracy of $a$.

Locally Lipschitz function $\boldsymbol{h}$. We now extend the analysis to functions satisfying conditions of Theorem 5 (thus almost everywhere differentiable), assuming additionally $\left(\mathcal{H}_{x_{0}}^{a}\right)$ : observe that the pointwise ellipticity condition $\int_{0}^{T} a^{2}\left(t, x_{0}\right) \mathrm{d} t>0$ is necessary to ensure that $X^{P}$-Greeks are well defined. The analysis below shows that the condition is also sufficient to obtain the expansion.

The new ingredient consists in appropriately smoothing $h$ and in using integrationby-parts formula from Malliavin calculus to get rid of the derivatives of $h$; this follows the arguments of [42]. Let $B$ be another scalar Brownian motion independent of $W$ and for $\delta>0$, set

$$
h_{\delta}(x):=\mathbb{E}\left(h\left(x+\delta B_{2 T}\right)\right)=\mathbb{E}\left(h_{\delta / \sqrt{2}}\left(x+\delta B_{T}\right)\right) .
$$

For any $\delta>0$, the function $h_{\delta}$ is smooth and its derivatives are exponentially bounded, so that we can apply the previous expansion to $h_{\delta}$ instead of $h$ in order to obtain:

$$
\begin{aligned}
\mathbb{E}\left[h_{\delta}\left(X_{T}\right)\right]= & \mathbb{E}\left[h_{\delta}\left(X_{T}^{P}\right)\right]+\left.C_{1}\left(a ; x_{0}\right)_{0}^{T}\left(\partial_{\varepsilon^{3}}^{3}-\frac{3}{2} \partial_{\varepsilon^{2}}^{2}+\frac{1}{2} \partial_{\varepsilon}\right) \mathbb{E}\left[h_{\delta}\left(X_{T}^{P}+\varepsilon\right)\right]\right|_{\varepsilon=0} \\
& +\operatorname{Error}_{2}\left(h_{\delta}\right) .
\end{aligned}
$$

Take $\delta=\mathcal{M}_{1}(a)\left[\mathcal{M}_{0}(a)\right]^{2} T$ : then replacing $\mathbb{E}\left(h_{\delta}\left(X_{T}\right)\right)$ and $\mathbb{E}\left(h_{\delta}\left(X_{T}^{P}\right)\right)$ by $\mathbb{E}\left(h\left(X_{T}\right)\right)$ and $\mathbb{E}\left(h\left(X_{T}^{P}\right)\right)$ readily yields an extra error $\mathcal{O}\left(\mathcal{M}_{1}(a)\left[\mathcal{M}_{0}(a)\right]^{2} T^{\frac{3}{2}}\right)$ which has the right magnitude regarding the expected global error. Moreover using $\left(\mathcal{H}_{x_{0}}^{a}\right)$, we can also prove that computing the sensitivities with respect to $h$ or to $h_{\delta}$ does not deteriorate the global accuracy (see [42, Lemma 4.2]). It remains to prove that $\operatorname{Error}_{2}\left(h_{\delta}\right)=\mathcal{O}\left(\mathcal{M}_{1}(a)\left[\mathcal{M}_{0}(a)\right]^{2} T^{\frac{3}{2}}\right)$. An inspection of the representation (42) of Error $_{2}\left(h_{\delta}\right)$ shows immediately that the first contribution with $h_{\delta}^{(1)}$ is a $\mathcal{O}\left(\mathcal{M}_{1}(a)\left[\mathcal{M}_{0}(a)\right]^{2} T^{\frac{3}{2}}\right)$, by simply using the exponential growth condition on $h^{(1)}$ and the finiteness of exponential moments of $X_{T}^{\eta}$. The second contribution with $h_{\delta}^{(2)}$ 
is the integral over $\left(\eta_{1}, \eta_{2}, \lambda\right) \in[0,1]^{3}$ of $\left(1-\eta_{1}\right)\left(1-\eta_{2}\right)(1-\lambda)$ times

$$
\begin{aligned}
& \mathbb{E}\left[h_{\delta}^{(2)}\left(X_{T}^{P}+\lambda\left(X_{T}-X_{T}^{P}\right)\right) X_{2, T}^{\eta_{1}} X_{2, T}^{\eta_{2}}\right] \\
& =\mathbb{E}\left[h_{\delta / \sqrt{2}}^{(2)}\left(X_{T}^{P}+\lambda\left(X_{T}-X_{T}^{P}\right)+\delta B_{T}\right) X_{2, T}^{\eta_{1}} X_{2, T}^{\eta_{2}}\right] \\
& =\mathbb{E}\left[h_{\delta / \sqrt{2}}^{(1)}\left(X_{T}^{P}+\lambda\left(X_{T}-X_{T}^{P}\right)+\delta B_{T}\right) H_{1}^{\delta, \eta_{1}, \eta_{2}, \lambda}\right] .
\end{aligned}
$$

The first equality follows from the definition of $h_{\delta}$, whereas the second one is an integration by parts formula from Malliavin calculus [48, Proposition 2.1.4]. We do not enter into the derivation details, we only emphasize two points: first, it is allowed since $X_{T}^{P}+\lambda\left(X_{T}-X_{T}^{P}\right)+\delta B_{T}$ is a non-degenerate random variable (in Malliavin sense) thanks to the additional perturbation $\delta B_{T}$, and its Malliavin matrix has an inverse of order $\left(\int_{0}^{T} a^{2}\left(t, x_{0}\right) \mathrm{d} t\right)^{-1}$ in $L_{p}$-norms, owing to the ellipticity assumption in $\left(\mathcal{H}_{x_{0}}^{a}\right)$. Second, the Malliavin norms of $X_{2, T}^{\eta}$ can be estimated similarly to (43) and it finally gives that $\left(\mathbb{E}\left|H_{1}^{\delta, \eta_{1}, \eta_{2}, \lambda}\right|^{2}\right)^{1 / 2}=\mathcal{O}\left(\mathcal{M}_{1}(a)\left[\mathcal{M}_{0}(a)\right]^{2} T^{\frac{3}{2}}\right)$. This finishes the proof. Slight modifications in the above arguments would enable to handle functions with local Hölder smoothness.

Arbitrary function $\boldsymbol{h}$. Here, we do not assume any regularity on $h$, only exponential growth. The analysis is similar but the regularization step for $h$ is more complex, see [45]: the expansion analysis has been done under the uniform ellipticity condition on $\left(\mathcal{H}^{a}\right)$, and not only under the pointwise ellipticity in $\left(\mathcal{H}_{x_{0}}^{a}\right)$.

As a conclusion to this stochastic analysis approach:

- the derivation of expansion coefficients is direct and easy;

- the error analysis relies on delicate Malliavin calculus estimates;

- it applies to general function $h$ under mild non-degeneracy condition.

\subsection{Mixing stochastic analysis and PDE}

Here, we directly prove the expansion result for locally Lipschitz function $h$. We represent the error $\mathbb{E}\left[h\left(X_{T}\right)\right]-\mathbb{E}\left[h\left(X_{T}^{P}\right)\right]$ using the PDE associated to the proxy:

$$
u^{P, h}(t, x)=\mathbb{E}\left[h\left(X_{T}^{P}\right) \mid X_{t}^{P}=x\right]
$$

To get a smooth solution $u^{P}$, assume that $a\left(t, x_{0}\right) \neq 0$ for any $t \in[0, T]$, which is stronger that $\int_{0}^{T} a^{2}\left(t, x_{0}\right) \mathrm{d} t>0$ considered in $\left(\mathcal{H}_{x_{0}}^{a}\right)$. The generic constants appearing in our next error estimates depend in an increasing way of the oscillation ratio $\frac{\mathcal{M}_{0}(a)}{\inf _{t \in[0, T]} a\left(t, x_{0}\right)}$. Then,

$$
\begin{aligned}
& \left\{\begin{array}{l}
\partial_{t} u^{P, h}(t, x)+\frac{1}{2} a^{2}\left(t, x_{0}\right)\left(\partial_{x^{2}}^{2}-\partial_{x}\right) u^{P, h}(t, x)=0, \quad \text { for } t<T, \\
u^{P, h}(T, x)=h(x),
\end{array}\right. \\
& \left|\partial_{x^{n}}^{n} u^{P, h}(t, x)\right| \leq_{c} e^{c|x|}\left(\int_{t}^{T} a^{2}\left(s, x_{0}\right) \mathrm{d} s\right)^{-\frac{n-1}{2}} .
\end{aligned}
$$


The estimates (45) directly follow from the differentiation of the Gaussian density of $X_{T}^{P}$ conditionally to $X_{t}^{p}=x$, taking into account the exponential growth of $h$. Then, apply Itô's formula to $u^{P, h}\left(t, X_{t}\right)$ between $t=0$ and $t=T$, combine this with simplifications coming from the PDE solved by $u^{P, h}$; it gives

$$
\begin{aligned}
\mathbb{E}\left[h\left(X_{T}\right)\right]=\mathbb{E}\left[h\left(X_{T}^{P}\right)\right]+\frac{1}{2} \mathbb{E}\left[\int_{0}^{T}\left(a^{2}\left(t, X_{t}\right)-a^{2}\left(t, x_{0}\right)\right)\left(\partial_{x^{2}}^{2}-\partial_{x}\right) u^{P, h}\left(t, X_{t}\right) \mathrm{d} t\right] \\
=\mathbb{E}\left[h\left(X_{T}^{P}\right)\right]+\frac{1}{2} \int_{0}^{T} \partial_{x}\left[a^{2}\right]\left(t, x_{0}\right) \mathbb{E}\left[\left(X_{t}-x_{0}\right)\left(\partial_{x^{2}}^{2}-\partial_{x}\right) u^{P, h}\left(t, X_{t}\right)\right] \mathrm{d} t \\
+\frac{1}{2} \mathbb{E}\left[\int_{0}^{T}\left[a^{2}\left(t, X_{t}\right)-a^{2}\left(t, x_{0}\right)-\partial_{x}\left[a^{2}\right]\left(t, x_{0}\right)\left(X_{t}-x_{0}\right)\right]\right. \\
\left.\quad \times\left(\partial_{x x}^{2}-\partial_{x}\right) u^{P, h}\left(t, X_{t}\right) \mathrm{d} t\right]
\end{aligned}
$$

Taking advantage of (45), we can easily bound the last term in (46) by

$$
C \int_{0}^{T} \mathcal{M}_{0}(a) \mathcal{M}_{1}(a)\left|X_{t}-x_{0}\right|_{4}^{2}\left(\int_{t}^{T} a^{2}\left(s, x_{0}\right) \mathrm{d} s\right)^{-\frac{1}{2}} \mathrm{~d} t=\mathcal{O}\left(\mathcal{M}_{1}(a)\left[\mathcal{M}_{0}(a)\right]^{2} T^{\frac{3}{2}}\right)
$$

using standard increment estimates and uniform lower and upper bounds on $a^{2}$. Observe that the Lipschitz regularity of $h$ gives rise to singular terms of the form $(T-t)^{-\frac{1}{2}}$, which are fortunately integrable at $T$.

Regarding the second term in (46), we have to approximate $\mathbb{E}\left[\left(X_{t}-x_{0}\right)\left(\partial_{x^{2}}^{2}-\right.\right.$ $\left.\left.\partial_{x}\right) u^{P, h}\left(t, X_{t}\right)\right]$ for any $t \in[0, T[$ : we apply again the previous decomposition by replacing $T$ by $t$ and $h(x)$ by $\phi_{t}(x)=\left(x-x_{0}\right)\left(\partial_{x x}^{2}-\partial_{x}\right) u^{P, h}(t, x)$. We denote by $v_{t}^{P, \phi}(s, x)=\mathbb{E}\left[\phi_{t}\left(X_{t}^{P}\right) \mid X_{s}^{P}=x\right]$ the solution of the system (44) on $[0, t[\times \mathbb{R}$ but with terminal condition $\phi_{t}$. The term under study is thus equal to

$$
\mathbb{E}\left[\phi_{t}\left(X_{t}^{P}\right)\right]+\frac{1}{2} \mathbb{E}\left[\int_{0}^{t}\left(a^{2}\left(s, X_{s}\right)-a^{2}\left(s, x_{0}\right)\right)\left(\partial_{x^{2}}^{2}-\partial_{x}\right) v_{t}^{P, \phi}\left(s, X_{s}\right) \mathrm{d} s\right]
$$

It remains to make explicit $v_{t}^{P, \phi}(s, x)$ in order to compute the first term and to estimate the second. For this, the trick lies in the observation that for any $k \geq$ $0, M_{k, t}=\partial_{x^{k}}^{k} u^{P, h}\left(t, X_{t}^{P}\right)$ is a martingale for $t<T$ : this directly follows from the application of Itô's formula, combined with (44) and (45). Hence, successive applications of the equalities $\mathbb{E}\left[M_{k, t} \mid X_{s}^{P}=x\right]=\partial_{x^{k}}^{k} u^{P, h}(s, x)$ for $s \leq t$ and of the Lemma 4 gives:

$$
\begin{aligned}
v_{t}^{P, \phi}(s, x)= & \mathbb{E}\left[\left(X_{t}^{P}-x_{0}\right)\left(\partial_{x^{2}}^{2}-\partial_{x}\right) u^{P, h}\left(t, X_{t}^{P}\right) \mid X_{s}^{P}=x\right] \\
= & \left(x-x_{0}\right) \mathbb{E}\left[\left(\partial_{x^{2}}^{2}-\partial_{x}\right) u^{P, h}\left(t, X_{t}^{P}\right) \mid X_{s}^{P}=x\right] \\
& +\mathbb{E}\left[\left(X_{t}^{P}-X_{s}^{P}\right)\left(\partial_{x^{2}}^{2}-\partial_{x}\right) u^{P, h}\left(t, X_{t}^{P}\right) \mid X_{s}^{P}=x\right]
\end{aligned}
$$




$$
\begin{aligned}
= & \left(x-x_{0}\right)\left(\partial_{x^{2}}^{2}-\partial_{x}\right) u^{P, h}(s, x)-\frac{1}{2} \int_{s}^{t} a^{2}\left(\xi, x_{0}\right) \mathrm{d} \xi \mathbb{E}\left[\left(\partial_{x^{2}}^{2}-\partial_{x}\right) u^{P, h}\left(t, X_{t}^{P}\right) \mid X_{s}^{P}=x\right] \\
& +\mathbb{E}\left[\left(\int_{s}^{t} a\left(\xi, x_{0}\right) \mathrm{d} W_{\xi}\right)\left(\partial_{x^{2}}^{2}-\partial_{x}\right) u^{P, h}\left(t, x-\frac{1}{2} \int_{s}^{t} a^{2}\left(\xi, x_{0}\right) \mathrm{d} \xi+\int_{s}^{t} a\left(\xi, x_{0}\right) \mathrm{d} W_{\xi}\right)\right] \\
= & \left(x-x_{0}\right)\left(\partial_{x^{2}}^{2}-\partial_{x}\right) u^{P, h}(s, x)-\frac{1}{2} \int_{s}^{t} a^{2}\left(\xi, x_{0}\right) \mathrm{d} \xi\left(\partial_{x^{2}}^{2}-\partial_{x}\right) u^{P, h}(s, x) \\
& +\int_{s}^{t} a^{2}\left(\xi, x_{0}\right) \mathrm{d} \xi \mathbb{E}\left[\left(\partial_{x^{3}}^{3}-\partial_{x^{2}}^{2}\right) u^{P, h}\left(t, X_{t}^{P}\right) \mid X_{s}^{P}=x\right] \\
= & \left(x-x_{0}\right)\left(\partial_{x^{2}}^{2}-\partial_{x}\right) u^{P, h}(s, x)+\int_{s}^{t} a^{2}\left(\xi, x_{0}\right) \mathrm{d} \xi\left(\partial_{x^{3}}^{3}-\frac{3}{2} \partial_{x^{2}}^{2}+\frac{1}{2} \partial_{x}\right) u^{P, h}(s, x) .
\end{aligned}
$$

In particular the above calculus yields $\mathbb{E}\left[\phi_{t}\left(X_{t}^{P}\right)\right]=v_{t}^{P, \phi}\left(0, x_{0}\right)=$ $\int_{0}^{t} a^{2}\left(s, x_{0}\right) \mathrm{d} s\left(\partial_{x^{3}}^{3}-\frac{3}{2} \partial_{x^{2}}^{2}+\frac{1}{2} \partial_{x}\right) u^{P, h}\left(0, x_{0}\right)$ and by multiplying by $\frac{1}{2} \partial_{x}\left[a^{2}\right]\left(t, x_{0}\right)$ and integrating over $t \in[0, T]$ in (46), we recover the correction terms from Theorem 5 .

On the other hand, combining (45) and the ellipticity assumption, we easily obtain

$$
\begin{aligned}
\left|\left(\partial_{x^{2}}^{2}-\partial_{x}\right) v_{t}^{P, \phi}\left(s, X_{s}\right)\right|_{p} \leq_{c}\left|X_{s}-x_{0}\right|_{2 p}\left|\left(\partial_{x^{4}}^{4}-2 \partial_{x^{3}}^{3}+\partial_{x^{2}}^{2}\right) u^{P, h}\left(s, X_{s}\right)\right|_{2 p} \\
+\left|\left(2 \partial_{x^{3}}^{3}-3 \partial_{x^{2}}^{2}+\partial_{x}\right) u^{P, h}\left(s, X_{s}\right)\right|_{p} \\
\quad+\int_{s}^{t} a^{2}\left(\xi, x_{0}\right) \mathrm{d} \xi\left|\left(\partial_{x^{5}}^{5}-\frac{5}{2} \partial_{x^{4}}^{4}+2 \partial_{x^{3}}^{3}-\frac{1}{2} \partial_{x^{2}}^{2}\right) u^{P, h}\left(s, X_{s}\right)\right|_{p} \\
\leq \leq_{c} \frac{\sqrt{s}}{\inf _{s \in[0, T]} a^{2}\left(s, x_{0}\right)(T-s)^{\frac{3}{2}}}+\frac{1}{\inf _{s \in[0, T]} a^{2}\left(s, x_{0}\right)(T-s)},
\end{aligned}
$$

for any $p \geq 1, t \in[0, T[, s \in[0, t[$. Consequently we obtain for the final error:

$$
\begin{aligned}
& \left|\int_{0}^{T} \partial_{x}\left[a^{2}\right]\left(t, x_{0}\right) \mathbb{E}\left[\int_{0}^{t}\left(a^{2}\left(s, X_{s}\right)-a^{2}\left(s, x_{0}\right)\right)\left(\partial_{x^{2}}^{2}-\partial_{x}\right) v_{t}^{P, \phi}\left(s, X_{s}\right) \mathrm{d} s\right] \mathrm{d} t\right| \\
\leq & { }_{c} \mathcal{M}_{1}(a) \mathcal{M}_{0}(a) \int_{0}^{T} \int_{0}^{t}\left|X_{s}-x_{0}\right|_{2}\left[\frac{\sqrt{s}}{(T-s)^{\frac{3}{2}}}+\frac{1}{(T-s)}\right] \mathrm{d} s \mathrm{~d} t \\
\leq & { }_{c} \mathcal{M}_{1}(a)\left[\mathcal{M}_{0}(a)\right]^{2} T^{\frac{3}{2}} .
\end{aligned}
$$

We have retrieved the error estimate provided in Theorem 5 . Once again, we would like to point out that the singular terms $(T-s)^{-3 / 2}$ and $(T-s)^{-1}$ appearing in the above time iterated integral remain integrable.

As a conclusion to this approach mixing stochastic analysis and PDE:

- the error analysis relies on usual estimates of derivatives of heat equations (PDE satisfied by the proxy) and it may be considered easier; however for digital options, the singularities arising in iterated time integrals are not integrable and the current approach seems to be inappropriate. 
- this approach requires stronger non-degeneracy assumptions compared to the previous stochastic analysis approach;

- the explicit derivation of expansion coefficients is tricky and relies on appropriate combination of martingale properties and Itô calculus;

- we nevertheless mention that this approach could be potentially used in a framework where the Malliavin calculus fails, e.g. for barrier options.

Actually for higher order expansion, the latter explicit martingale computation is harder to write down, whereas a direct application of Lemma 4 remains direct.

\subsection{A pure PDE approach}

Alternatively, inspired by the interpolation (23-24), consider the solution of the PDE

$$
\begin{cases}\partial_{t} u^{\eta}(t, x)+\frac{1}{2} a^{2}\left(t, x_{0}+\eta\left(x-x_{0}\right)\right)\left(\partial_{x^{2}}^{2}-\partial_{x}\right) u^{\eta}(t, x)=0, & \text { for } t<T, \\ u^{\eta}(T, x)=h(x) . & \end{cases}
$$

Observe that $u^{1}\left(0, x_{0}\right)$ coincides with $\mathbb{E}\left(h\left(X_{T}\right)\right)$ whereas $u^{0}\left(0, x_{0}\right)$ coincides with $\mathbb{E}\left(h\left(X_{T}^{P}\right)\right)$. This PDE has similarities with that of Hagan (20) but it differs here, because the space variable has not been rescaled around the strike. In addition the solution of the principal PDE in the Hagan approach is a Call price in a Bachelier model, whereas $u^{0}\left(0, x_{0}\right)$ is a Call price in a Black-Scholes model.

To derive the correction terms, we shall apply a regular perturbation analysis by writing $u^{\eta}=u_{0}+\eta u_{1}+\ldots$, with $u_{0}=u^{0}$, and

$L^{\eta}=\partial_{t}+\frac{1}{2} a^{2}\left(t, x_{0}+\eta\left(x-x_{0}\right)\right)\left(\partial_{x^{2}}^{2}-\partial_{x}\right)=L_{0}+\eta \frac{1}{2} \partial_{x}\left[a^{2}\right]\left(t, x_{0}\right)\left(x-x_{0}\right)\left(\partial_{x^{2}}^{2}-\partial_{x}\right)+\ldots$

A formal identification of the system to PDEs to solve gives

$\left[\partial_{t}+\frac{1}{2} a^{2}\left(t, x_{0}\right)\left(\partial_{x^{2}}^{2}-\partial_{x}\right)\right] u_{0}(t, x)=0, \quad L_{0} u_{1}=-\frac{1}{2} \partial_{x}\left[a^{2}\right]\left(t, x_{0}\right)\left(x-x_{0}\right)\left(\partial_{x^{2}}^{2}-\partial_{x}\right) u_{0}$

with $u_{0}(T, x)=h(x)$ and $u_{1}(T, x)=0$. As mentioned before, in our opinion, an explicit resolution of $u_{1}$ is difficult to exhibit without knowing the solution. However, after tedious calculus involving Gaussian kernels and convolutions, we can retrieve the corrective terms of Theorem 5 .

Also, a PDE error analysis (which we have not been able to find in the literature in the case of irregular $h$ ) may presumably give error estimates only in powers of $\eta$ (which equals 1 here!) and not as $\mathcal{O}\left(\mathcal{M}_{1}(a)\left[\mathcal{M}_{0}(a)\right]^{2} T^{\frac{3}{2}}\right)$. Additionally, due to the form of the proxy, our intuition is that the error at $\left(0, x_{0}\right)$ (where we aim at computing the solution) is smaller that the error at arbitrary $(t, x)$. All these reasons indicate that a $\mathrm{PDE}$ approach to derive correction terms and error analysis in our proxy setting is probably irrelevant. 


\section{Higher-order proxy approximation}

In this section, we give several expansions formulas with a third order accuracy. First, we recall without proof results obtained in [49] and [44] for expansions based on local volatility at spot and at strike. Second we introduce a new expansion with local volatility frozen at mid-point. Finally new expansions of implied volatility are provided.

\subsection{Third order approximation with the local volatility at spot and at strike.}

We define some integral operators useful to state the next theorems.

Definition 17. If the derivatives and the integrals have a meaning, we define for a two variables function $l$ the above operators:

$$
\begin{aligned}
& C_{1}(l ; z)_{0}^{T}=\omega\left(l^{2}(z), l(z) l^{(1)}(z)\right)_{0}^{T}, \\
& C_{2}(l ; z)_{0}^{T}=\omega\left(l^{2}(z),\left(l^{(1)}(z)\right)^{2}+l(z) l^{(2)}(z)\right)_{0}^{T}, \\
& C_{3}(l ; z)_{0}^{T}=\omega\left(l^{2}(z), l^{2}(z),\left(l^{(1)}(z)\right)^{2}+l(z) l^{(2)}(z)\right)_{0}^{T}, \\
& C_{4}(l ; z)_{0}^{T}=\omega\left(l^{2}(z), l(z) l^{(1)}(z), l(z) l^{(1)}(z)\right)_{0}^{T} .
\end{aligned}
$$

We frequently use some linear combinations of these operators:

$$
\begin{array}{rlrl}
\eta_{1}(l ; z)_{0}^{T} & =\frac{C_{1}(l ; z)_{0}^{T}}{2}-\frac{C_{2}(l ; z)_{0}^{T}}{2}-\frac{C_{3}(l ; z)_{0}^{T}}{4}-\frac{C_{4}(l ; z)_{0}^{T}}{2}, \\
\eta_{2}(l ; z)_{0}^{T} & =-\frac{3 C_{1}(l ; z)_{0}^{T}}{2}+\frac{C_{2}(l ; z)_{0}^{T}}{2}+\frac{5 C_{3}(l ; z)_{0}^{T}}{4}+\frac{7 C_{4}(l ; z)_{0}^{T}}{2}+\frac{\left[C_{1}(l ; z)_{0}^{T}\right]^{2}}{8}, \\
\eta_{3}(l ; z)_{0}^{T} & =C_{1}(l ; z)_{0}^{T}-2 C_{3}(l ; z)_{0}^{T}-6 C_{4}(l ; z)_{0}^{T}-\frac{3\left[C_{1}(l ; z)_{0}^{T}\right]^{2}}{4}, \\
\eta_{4}(l ; z)_{0}^{T} & =C_{3}(l ; z)_{0}^{T}+3 C_{4}(l ; z)_{0}^{T}+\frac{13\left[C_{1}(l ; z)_{0}^{T}\right]^{2}}{8}, \\
\eta_{5}(l ; z)_{0}^{T} & =-\frac{3\left[C_{1}(l ; z)_{0}^{T}\right]^{2}}{2}, \\
\eta_{6}(l ; z)_{0}^{T} & =\frac{\left[C_{1}(l ; z)_{0}^{T}\right]^{2}}{2}, \\
\zeta_{2}(l ; z)_{0}^{T} & =\frac{C_{2}(l ; z)_{0}^{T}}{2}, & \zeta_{3}(l ; z)_{0}^{T} & =C_{1}(l ; z)_{0}^{T}, \\
\zeta_{4}(l ; z)_{0}^{T} & =C_{3}(l ; z)_{0}^{T}+3 C_{4}(l ; z)_{0}^{T}, & \zeta_{6}(l ; z)_{0}^{T} & =\frac{\left[C_{1}(l ; z)_{0}^{T}\right]^{2}}{2} .
\end{array}
$$

Theorem 18. (3rd order approximations for Call options with the local volatility at spot). Assuming $\left(\mathcal{H}^{a}\right)$ and using the log-normal proxy, one has

$$
\begin{aligned}
\operatorname{Call}\left(e^{x_{0}}, T, e^{k}\right)= & \operatorname{Call}^{\mathrm{BS}}\left(x_{0}, \bar{a}_{x_{0}}^{2} T, k\right)+\sum_{i=1}^{6} \eta_{i}\left(a ; x_{0}\right)_{0}^{T} \partial_{x^{i}}^{i} \operatorname{Call}^{\mathrm{BS}}\left(x_{0}, \bar{a}_{x_{0}}^{2} T, k\right) \\
& +\mathcal{O}\left(\mathcal{M}_{1}(a)\left[\mathcal{M}_{0}(a)\right]^{3} T^{2}\right) .
\end{aligned}
$$


Assuming $\left(\mathcal{H}^{\Sigma}\right)$ and using the normal proxy, one has

$$
\begin{aligned}
\operatorname{Call}\left(S_{0}, T, K\right)= & \operatorname{Call}^{\mathrm{BA}}\left(S_{0}, \bar{\Sigma}_{S_{0}}^{2} T, K\right)+\sum_{i \in\{2,3,4,6\}} \zeta_{i}\left(\Sigma ; S_{0}\right)_{0}^{T} \partial_{S^{i}}^{i} \operatorname{Call}^{\mathrm{BA}}\left(S_{0}, \bar{\Sigma}_{S_{0}}^{2} T, K\right) \\
& +\mathcal{O}\left(\mathcal{M}_{1}(\Sigma)\left[\mathcal{M}_{0}(\Sigma)\right]^{3} T^{2}\right) .
\end{aligned}
$$

The operators $\zeta_{i}$ and $\eta_{i}$ in the above expansions are defined in Definition $1 \%$.

The magnitude of the residual terms in the previous formulas justifies the label of third order approximations.

The above theorem is a straightforward application of [49, Theorems 2.2, 2.3 and 4.2], taking into account that we slightly modify the notations of the Greek coefficients. Namely, for convenience we merge certain $\omega$ operators: for instance the reader can easily check that:

$$
\begin{aligned}
{\left[C_{1}(l ; z)_{0}^{T}\right]^{2}=\left[\omega\left(l(z)^{2}, l(z) l^{(1)}(z)\right)_{0}^{T}\right]^{2}=} & 4 \omega\left(l(z)^{2}, l(z)^{2}, l(z) l^{(1)}(z), l(z) l^{(1)}(z)\right)_{0}^{T} \\
& +2 \omega\left(l(z)^{2}, l(z) l^{(1)}(z), l(z)^{2}, l(z) l^{(1)}(z)\right)_{0}^{T} .
\end{aligned}
$$

We should mention that it seems possible to relax the strong hypothesis $\left(\mathcal{H}^{a}\right)$ which appears in [49, Theorems 2.2 and 4.2]. As for the second order approximations, $\left(\mathcal{H}_{x_{0}}^{a}\right)$ may be sufficient.

Using the duality argument introduced in Subsection 3.3 and [49, Theorems 2.2, 2.3 and 4.2 ], approximations using the volatility at strike are available too.

Theorem 19. (3rd order approximations for Call options with the local volatility at strike). Assuming $\left(\mathcal{H}^{a}\right)$ and using the log-normal proxy, one has

$$
\begin{aligned}
\operatorname{Call}\left(e^{x_{0}}, T, e^{k}\right)= & \operatorname{Call}^{\mathrm{BS}}\left(x_{0}, \bar{a}_{k}^{2} T, k\right)+\sum_{i=1}^{6} \eta_{i}(\widetilde{a} ; k)_{0}^{T} \partial_{z^{i}}^{i} \operatorname{Call}^{\mathrm{BS}}\left(x_{0}, \bar{a}_{k}^{2} T, k\right) \\
& +\mathcal{O}\left(\mathcal{M}_{1}(a)\left[\mathcal{M}_{0}(a)\right]^{3} T^{2}\right),
\end{aligned}
$$

Assuming $\left(\mathcal{H}^{\Sigma}\right)$ and using the normal proxy, one has

$$
\begin{aligned}
\operatorname{Call}\left(S_{0}, T, K\right)= & \operatorname{Call}^{\mathrm{BA}}\left(S_{0}, \bar{\Sigma}_{K}^{2} T, K\right)+\sum_{i \in\{2,3,4,6\}} \zeta_{i}(\widetilde{\Sigma} ; K)_{0}^{T} \partial_{Z^{i}}^{i} \operatorname{Call}^{\mathrm{BA}}\left(S_{0}, \bar{\Sigma}_{K}^{2} T, K\right) \\
& +\mathcal{O}\left(\mathcal{M}_{1}(\Sigma)\left[\mathcal{M}_{0}(\Sigma)\right]^{3} T^{2}\right) .
\end{aligned}
$$

The operators $\zeta_{i}$ and $\eta_{i}$ in the above expansions are defined in Definition $1 \%$.

\subsection{Third order approximation with the local volatility at mid- point.}

We now state a new result related to third order expansions based on the local volatility at mid-point $x_{\mathrm{av}}$ or $S_{\mathrm{av}}$. For a clearer proof, we change the presentation of the corrective terms in comparison with Theorems 18 and 19: instead of gathering 
them according the order of the Greeks, we put them together according to the operators $C_{i}$ introduced in Definition 17.

Theorem 20. (3rd order approximations for Call options with the local volatility at mid-point). Assuming $\left(\mathcal{H}^{a}\right)$ and using the log-normal proxy, one has

$$
\begin{aligned}
& \text { Call }\left(e^{x_{0}}, T, e^{k}\right)=\operatorname{Call}^{\mathrm{BS}}\left(x_{0}, \bar{a}_{x_{\mathrm{av}}}^{2} T, k\right) \\
& +\frac{C_{1}\left(a ; x_{\mathrm{av}}\right)_{0}^{T}-C_{1}\left(\widetilde{a} ; x_{\mathrm{av}}\right)_{0}^{T}}{2}\left(\partial_{x^{3}}^{3}-\frac{3}{2} \partial_{x^{2}}^{2}+\frac{1}{2} \partial_{x}\right) \mathrm{Call}^{\mathrm{BS}}\left(x_{0}, \bar{a}_{x_{\mathrm{av}}}^{2} T, k\right) \\
& +\frac{C_{2}\left(a ; x_{\mathrm{av}}\right)_{0}^{T}+C_{2}\left(\widetilde{a} ; x_{\mathrm{av}}\right)_{0}^{T}}{2}\left(\frac{1}{2} \partial_{x^{2}}^{2}-\frac{1}{2} \partial_{x}\right) \mathrm{Call}^{\mathrm{BS}}\left(x_{0}, \bar{a}_{x_{\mathrm{av}}}^{2} T, k\right) \\
& +\frac{C_{3}\left(a ; x_{\mathrm{av}}\right)_{0}^{T}+C_{3}\left(\widetilde{a} ; x_{\mathrm{av}}\right)_{0}^{T}}{2}\left(\partial_{x^{4}}^{4}-2 \partial_{x^{3}}^{3}+\frac{5}{4} \partial_{x^{2}}^{2}-\frac{1}{4} \partial_{x}\right) \mathrm{Call}^{\mathrm{BS}}\left(x_{0}, \bar{a}_{x_{\mathrm{av}}}^{2} T, k\right) \\
& +\frac{C_{4}\left(a ; x_{\mathrm{av}}\right)_{0}^{T}+C_{4}\left(\widetilde{a} ; x_{\mathrm{av}}\right)_{0}^{T}}{2}\left(3 \partial_{x^{4}}^{4}-6 \partial_{x^{3}}^{3}+\frac{7}{2} \partial_{x^{2}}^{2}-\frac{1}{2} \partial_{x}\right) \mathrm{Call}^{\mathrm{BS}}\left(x_{0}, \bar{a}_{x_{\mathrm{av}}}^{2} T, k\right) \\
& +\frac{\left[C_{1}\left(a ; x_{\mathrm{av}}\right)_{0}^{T}\right]^{2}+\left[C_{1}\left(\widetilde{a} ; x_{\mathrm{av}}\right)_{0}^{T}\right]^{2}}{2} \\
& \quad \times\left(\frac{1}{2} \partial_{x^{6}}^{6}-\frac{3}{2} \partial_{x^{5}}^{5}+\frac{13}{8} \partial_{x^{4}}^{4}-\frac{3}{4} \partial_{x^{3}}^{3}+\frac{1}{8} \partial_{x^{2}}^{2}\right) \mathrm{Call}^{\mathrm{BS}}\left(x_{0}, \bar{a}_{x_{\mathrm{av}}}^{2} T, k\right) \\
& -\left(x_{0}-k\right)^{2} C_{5}\left(a ; x_{\mathrm{av}}\right)_{0}^{T}\left(\frac{1}{8} \partial_{x^{2}}^{2}-\frac{1}{8} \partial_{x}\right) \mathrm{Call}^{\mathrm{BS}}\left(x_{0}, \bar{a}_{x_{\mathrm{av}}}^{2} T, k\right) \\
& +\left(x_{0}-k\right)^{2} C_{6}\left(a ; x_{\mathrm{av}}\right)_{0}^{T}\left(\frac{1}{4} \partial_{x^{4}}^{4}-\frac{1}{2} \partial_{x^{3}}^{3}+\frac{1}{4} \partial_{x^{2}}^{2}\right) \mathrm{Call}^{\mathrm{BS}}\left(x_{0}, \bar{a}_{x_{\mathrm{av}}}^{2} T, k\right) \\
& +\mathcal{O}\left(\mathcal{M}_{1}(a)\left[\mathcal{M}_{0}(a)\right]^{3} T^{2}\right),
\end{aligned}
$$

where the operators $C_{i}$ for $i=1 . .4$ are defined in Definition 17 and where the time reversal invariant $t^{\mathrm{e}}$ operators $C_{5}$ and $C_{6}$ are defined by:

$$
C_{5}(l ; z)_{0}^{T}=\omega\left(\left(l^{(1)}(z)\right)^{2}+l(z) l^{(2)}(z)\right)_{0}^{T}, \quad C_{6}(l ; z)_{0}^{T}=\omega\left(l(z) l^{(1)}(z), l(z) l^{(1)}(z)\right)_{0}^{T} .
$$

Assuming $\left(\mathcal{H}^{\Sigma}\right)$ and using the normal proxy, one has

$$
\begin{aligned}
\operatorname{Call}\left(S_{0}, T, K\right)= & \operatorname{Call}^{\mathrm{BA}}\left(S_{0}, \bar{\Sigma}_{S_{\mathrm{av}}}^{2} T, K\right) \\
& +\frac{C_{1}\left(\Sigma ; S_{\mathrm{av}}\right)_{0}^{T}-C_{1}\left(\widetilde{\Sigma} ; S_{\mathrm{av}}\right)_{0}^{T}}{2} \partial_{S^{3}}^{3} \operatorname{Call}^{\mathrm{BA}}\left(S_{0}, \bar{\Sigma}_{S_{\mathrm{av}}}^{2} T, K\right) \\
& +\frac{C_{2}\left(\Sigma ; S_{\mathrm{av}}\right)_{0}^{T}+C_{2}\left(\widetilde{\Sigma} ; S_{\mathrm{av}}\right)_{0}^{T}}{4} \partial_{S^{2}}^{2} \operatorname{Call}^{\mathrm{BA}}\left(S_{0}, \bar{\Sigma}_{S_{\mathrm{av}}}^{2} T, K\right) \\
& +\frac{C_{3}\left(\Sigma ; S_{\mathrm{av}}\right)_{0}^{T}+C_{3}\left(\widetilde{\Sigma} ; S_{\mathrm{av}}\right)_{0}^{T}}{2} \partial_{S^{4}}^{4}\left(S_{0}, \bar{\Sigma}_{S_{\mathrm{av}}}^{2} T, K\right) \\
& +3 \frac{C_{4}\left(\Sigma ; S_{\mathrm{av}}\right)_{0}^{T}+C_{4}\left(\widetilde{\Sigma} ; S_{\mathrm{av}}\right)_{0}^{T}}{2} \partial_{S^{4}}^{4}\left(S_{0}, \bar{\Sigma}_{S_{\mathrm{av}}}^{2} T, K\right)
\end{aligned}
$$

ethat is $C_{5}(\tilde{l} ; z)_{0}^{T}=C_{5}(l ; z)_{0}^{T}$ and $C_{6}(\tilde{l} ; z)_{0}^{T}=C_{6}(l ; z)_{0}^{T}$ using $(26)$. 


$$
\begin{aligned}
& +\frac{\left[C_{1}\left(\Sigma ; S_{\mathrm{av}}\right)_{0}^{T}\right]^{2}+\left[C_{1}\left(\widetilde{\Sigma} ; S_{\mathrm{av}}\right)_{0}^{T}\right]^{2}}{4} \partial_{S^{6}}^{6}\left(S_{0}, \bar{\Sigma}_{S_{\mathrm{av}}}^{2} T, K\right) \\
& -\left(x_{0}-k\right)^{2} \frac{C_{5}\left(\Sigma ; S_{\mathrm{av}}\right)_{0}^{T}}{8} \partial_{S^{2}}^{2} \operatorname{Call}^{\mathrm{BA}}\left(S_{0}, \bar{\Sigma}_{S_{\mathrm{av}}}^{2} T, K\right) \\
& -\left(x_{0}-k\right)^{2} \frac{C_{6}\left(\Sigma ; S_{\mathrm{av}}\right)_{0}^{T}}{4} \partial_{S^{4}}^{4}\left(S_{0}, \bar{\Sigma}_{S_{\mathrm{av}}}^{2} T, K\right) \\
& +\mathcal{O}\left(\mathcal{M}_{1}(\Sigma)\left[\mathcal{M}_{0}(\Sigma)\right]^{3} T^{2}\right) .
\end{aligned}
$$

Proof. We only prove the result for the log-normal proxy. The case of normal proxy is similar, and it is left to the reader as an exercise. The idea is again to consider the average of the third order formulas in spot and strike provided in Theorems 18 and 19 and to perform an expansion around the mid-point.

$\triangleright$ Step 1: expansion of the leading term. Firstly we aim at showing that:

$$
\begin{aligned}
& \left(\text { Call }^{\mathrm{BS}}\left(x_{0}, \bar{a}_{x_{0}}^{2} T, k\right)+\mathrm{Call}^{\mathrm{BS}}\left(x_{0}, \bar{a}_{k}^{2} T, k\right)\right) / 2 \\
& =\mathrm{Call}^{\mathrm{BS}}\left(x_{0}, \bar{a}_{x_{\mathrm{av}}}^{2} T, k\right)+\frac{\left(x_{0}-k\right)^{2}}{4} C_{5}\left(a ; x_{\mathrm{av}}\right)_{0}^{T} \partial_{y} \mathrm{Call}^{\mathrm{BS}}\left(x_{0}, \bar{a}_{x_{\mathrm{av}}}^{2} T, k\right) \\
& \quad+\left(x_{0}-k\right)^{2} C_{6}\left(a ; x_{\mathrm{av}}\right)_{0}^{T} \partial_{y^{2}}^{2} \mathrm{Call}^{\mathrm{BS}}\left(x_{0}, \bar{a}_{x_{\mathrm{av}}}^{2} T, k\right)+\mathcal{O}\left(\mathcal{M}_{1}(a)\left[\mathcal{M}_{0}(a)\right]^{3} T^{2}\right),
\end{aligned}
$$

where the operators $C_{5}$ and $C_{6}$ are defined in Theorem 20. Perform Taylor expansions to obtain:

$$
\begin{aligned}
\text { Call }^{\mathrm{BS}} & \left(x_{0}, \bar{a}_{x_{0}}^{2} T, k\right) \\
= & \text { Call }^{\mathrm{BS}}\left(x_{0}, \bar{a}_{x_{\mathrm{av}}}^{2} T, k\right)+\partial_{y} \operatorname{Call}^{\mathrm{BS}}\left(x_{0}, \bar{a}_{x_{\mathrm{av}}}^{2} T, k\right) T\left(\bar{a}_{x_{0}}^{2}-\bar{a}_{x_{\mathrm{av}}}^{2}\right) \\
& +\frac{1}{2} \partial_{y^{2}} \mathrm{Call}^{\mathrm{BS}}\left(x_{0}, \bar{a}_{x_{\mathrm{av}}}^{2} T, k\right) T^{2}\left(\bar{a}_{x_{0}}^{2}-\bar{a}_{x_{\mathrm{av}}}^{2}\right)^{2}+R_{1} \\
= & \text { Call }^{\mathrm{BS}}\left(x_{0}, \bar{a}_{x_{\mathrm{av}}}^{2} T, k\right)+\partial_{y} \operatorname{Call}^{\mathrm{BS}}\left(x_{0}, \bar{a}_{x_{\mathrm{av}}}^{2} T, k\right) \omega\left(a\left(x_{\mathrm{av}}\right) a^{(1)}\left(x_{\mathrm{av}}\right)\right)_{0}^{T}\left(x_{0}-k\right) \\
& +\frac{1}{4} \partial_{y} \mathrm{Call}^{\mathrm{BS}}\left(x_{0}, \bar{a}_{x_{\mathrm{av}}}^{2} T, k\right) C_{5}\left(a ; x_{\mathrm{av}}\right)_{0}^{T}\left(x_{0}-k\right)^{2} \\
& +\partial_{y^{2}}^{2} \mathrm{Call}^{\mathrm{BS}}\left(x_{0}, \bar{a}_{x_{\mathrm{av}}}^{2} T, k\right) C_{6}\left(a ; x_{\mathrm{av}}\right)_{0}^{T}\left(x_{0}-k\right)^{2}+R_{1}+R_{2}+R_{3},
\end{aligned}
$$

where we have used the relations $\partial_{z} \omega\left(l^{2}(z)\right)_{0}^{T}=2 \omega\left(l(z) l^{(1)}(z)\right)_{0}^{T}, \partial_{z^{2}}^{2} \omega\left(l^{2}(z)\right)_{0}^{T}=$ $2 C_{5}(l ; z)_{0}^{T},\left[\omega\left(l(z) l^{(1)}(z)\right)_{0}^{T}\right]^{2}=2 C_{6}(l ; z)_{0}^{T}$ and where $R_{1}, R_{2}$ and $R_{3}$ are defined by:

$$
\begin{aligned}
R_{1}= & \left.T^{3}\left(\bar{a}_{x_{0}}^{2}-\bar{a}_{x_{\mathrm{av}}}^{2}\right)^{3} \int_{0}^{1}\left(\partial_{y^{3}}^{3} \mathrm{Call}^{\mathrm{BS}}\left(x_{0}, y, k\right)\right)\right|_{y=T\left(\lambda \bar{a}_{x_{0}}^{2}+(1-\lambda) \bar{a}_{x_{\mathrm{av}}}^{2}\right)} \frac{(1-\lambda)^{2}}{2} \mathrm{~d} \lambda, \\
R_{2}= & \left.\partial_{y} \operatorname{Call}^{\mathrm{BS}}\left(x_{0}, \bar{a}_{x_{\mathrm{av}}}^{2} T, k\right) \frac{\left(x_{0}-k\right)^{3}}{8} \int_{0}^{1}\left(\partial_{x}^{3} \omega\left(a^{2}(x)\right)_{0}^{T}\right)\right|_{x=\lambda x_{0}+(1-\lambda) x_{\mathrm{av}}} \frac{(1-\lambda)^{2}}{2} \mathrm{~d} \lambda, \\
R_{3}= & \frac{1}{2} \partial_{y^{2}}^{2} \mathrm{Call}^{\mathrm{BS}}\left(x_{0}, \bar{a}_{x_{\mathrm{av}}}^{2} T, k\right) \frac{\left(x_{0}-k\right)^{2}}{4} \int_{0}^{1}\left(\partial_{x}^{2} \omega\left(a^{2}(x)\right)_{0}^{T}\right)_{x=\lambda x_{0}+(1-\lambda) x_{\mathrm{av}}}(1-\lambda) \mathrm{d} \lambda \\
& \times\left[\frac{\left(x_{0}-k\right)^{2}}{4} \int_{0}^{1}\left(\partial_{x}^{2} \omega\left(a^{2}(x)\right)_{0}^{T}\right)_{x=\lambda x_{0}+(1-\lambda) x_{\mathrm{av}}}(1-\lambda) \mathrm{d} \lambda\right. \\
& \left.\quad+2 \omega\left(a\left(x_{\mathrm{av}}\right) a^{(1)}\left(x_{\mathrm{av}}\right)\right)_{0}^{T}\left(x_{0}-k\right)\right] .
\end{aligned}
$$


Using (6), Corollary 30 and $\left(\mathcal{H}^{a}\right)$ we obtain

$$
\left|R_{1}+R_{2}+R_{3}\right| \leq_{c} \mathcal{M}_{1}(a)\left[\mathcal{M}_{0}(a)\right]^{3} T^{2} .
$$

Similarly, we show that:

$$
\begin{aligned}
\text { Call }^{\mathrm{BS}} & \left(x_{0}, \bar{a}_{k}^{2} T, k\right) \\
= & \mathrm{Call}^{\mathrm{BS}}\left(x_{0}, \bar{a}_{x_{\mathrm{av}}}, k\right)-\partial_{y} \mathrm{Call}^{\mathrm{BS}}\left(x_{0}, \bar{a}_{x_{\mathrm{av}}}^{2} T, k\right) \omega\left(a\left(x_{\mathrm{av}}\right) a^{(1)}\left(x_{\mathrm{av}}\right)\right)_{0}^{T}\left(x_{0}-k\right) \\
& +\frac{1}{4} \partial_{y} \mathrm{Call}^{\mathrm{BS}}\left(x_{0}, \bar{a}_{x_{\mathrm{av}}}^{2} T, k\right) C_{5}\left(a ; x_{\mathrm{av}}\right)_{0}^{T}\left(x_{0}-k\right)^{2} \\
& +\partial_{y^{2}}^{2} \operatorname{Call}^{\mathrm{BS}}\left(x_{0}, \bar{a}_{x_{\mathrm{av}}}^{2} T, k\right) C_{6}\left(a ; x_{\mathrm{av}}\right)_{0}^{T}\left(x_{0}-k\right)^{2}+\mathcal{O}\left(\mathcal{M}_{1}(a)\left[\mathcal{M}_{0}(a)\right]^{3} T^{2}\right) .
\end{aligned}
$$

Combine this with (49) to obtain (48).

$\triangleright$ Step 2: expansion of the corrective terms. Firstly we treat the corrective terms with the operators $C_{2}, C_{3}, C_{4}$ and $\left[C_{1}\right]^{2}$ in Theorems 18 and 19 . We let the reader verify that in the formula with volatility at spot (respectively in strike), we can replace the point $x_{0}$ (respectively $k$ ) by the point $x_{\mathrm{av}}$ in all the corrective terms involving these operators: indeed it induces an extra error of order $\mathcal{M}_{1}(a)\left[\mathcal{M}_{0}(a)\right]^{3} T^{2}$. This is very similar to the proof of Lemma 11 so we skip it. Then we can replace derivatives w.r.t. $z$ with derivatives w.r.t. $x$ in Call ${ }^{\mathrm{BS}}$ thanks to Proposition 34, equations (72)-(74)-(75)-(76). That leads to:

$$
\begin{aligned}
& \frac{1}{2} C_{2}\left(a ; x_{0}\right)_{0}^{T}\left(\frac{1}{2} \partial_{x^{2}}^{2}-\frac{1}{2} \partial_{x}\right) \mathrm{Call}^{\mathrm{BS}}\left(x_{0}, \bar{a}_{x_{0}}^{2} T, k\right) \\
+ & \frac{1}{2} C_{2}(\widetilde{a} ; k)_{0}^{T}\left(\frac{1}{2} \partial_{z^{2}}^{2}-\frac{1}{2} \partial_{z}\right) \mathrm{Call}^{\mathrm{BS}}\left(x_{0}, \bar{a}_{k}^{2} T, k\right) \\
+ & \frac{1}{2} C_{3}\left(a ; x_{0}\right)_{0}^{T}\left(\partial_{x^{4}}^{4}-2 \partial_{x^{3}}^{3}+\frac{5}{4} \partial_{x^{2}}^{2}-\frac{1}{4} \partial_{x}\right) \mathrm{Call}^{\mathrm{BS}}\left(x_{0}, \bar{a}_{x_{0}}^{2} T, k\right) \\
+ & \frac{1}{2} C_{3}(\widetilde{a} ; k)_{0}^{T}\left(\partial_{z^{4}}^{4}-2 \partial_{z^{3}}^{3}+\frac{5}{4} \partial_{z^{2}}^{2}-\frac{1}{4} \partial_{z}\right) \mathrm{Call}^{\mathrm{BS}}\left(x_{0}, \bar{a}_{k}^{2} T, k\right) \\
+ & \frac{1}{2} C_{4}\left(a ; x_{0}\right)_{0}^{T}\left(3 \partial_{x^{4}}^{4}-6 \partial_{x^{3}}^{3}+\frac{7}{2} \partial_{x^{2}}^{2}-\frac{1}{2} \partial_{x}\right) \mathrm{Call}^{\mathrm{BS}}\left(x_{0}, \bar{a}_{x_{0}}^{2} T, k\right) \\
+ & \frac{1}{2} C_{4}(\widetilde{a} ; k)_{0}^{T}\left(3 \partial_{z^{4}}^{4}-6 \partial_{z^{3}}^{3}+\frac{7}{2} \partial_{z^{2}}^{2}-\frac{1}{2} \partial_{z}\right) \mathrm{Call}^{\mathrm{BS}}\left(x_{0}, \bar{a}_{k}^{2} T, k\right) \\
+ & \frac{1}{2}\left[C_{1}\left(a ; x_{0}\right)_{0}^{T}\right]^{2}\left(\frac{1}{2} \partial_{x^{6}}^{6}-\frac{3}{2} \partial_{x^{5}}^{5}+\frac{13}{8} \partial_{x^{4}}^{4}-\frac{3}{4} \partial_{x^{3}}^{3}+\frac{1}{8} \partial_{x^{2}}^{2}\right) \mathrm{Call}^{\mathrm{BS}}\left(x_{0}, \bar{a}_{x_{0}}^{2} T, k\right) \\
+ & \frac{1}{2}\left[C_{1}(\widetilde{a} ; k)_{0}^{T}\right]^{2}\left(\frac{1}{2} \partial_{z^{6}}^{6}-\frac{3}{2} \partial_{z^{5}}^{5}+\frac{13}{8} \partial_{z^{4}}^{4}-\frac{3}{4} \partial_{z^{3}}^{3}+\frac{1}{8} \partial_{z^{2}}^{2}\right) \mathrm{Call}^{\mathrm{BS}}\left(x_{0}, \bar{a}_{k}^{2} T, k\right) \\
= & \frac{C_{2}\left(a ; x_{\mathrm{av}}\right)_{0}^{T}+C_{2}\left(\widetilde{a} ; x_{\mathrm{av}}\right)_{0}^{T}}{2}\left(\frac{1}{2} \partial_{x^{2}}^{2}-\frac{1}{2} \partial_{x}\right) \mathrm{Call}^{\mathrm{BS}}\left(x_{0}, \bar{a}_{x_{\mathrm{av}}}^{2} T, k\right) \\
+ & \frac{C_{3}\left(a ; x_{\mathrm{av}}\right)_{0}^{T}+C_{3}\left(\widetilde{a} ; x_{\mathrm{av}}\right)_{0}^{T}}{2}\left(\partial_{x^{4}}^{4}-2 \partial_{x^{3}}^{3}+\frac{5}{4} \partial_{x^{2}}^{2}-\frac{1}{4} \partial_{x}\right) \mathrm{Call}^{\mathrm{BS}}\left(x_{0}, \bar{a}_{x_{\mathrm{av}}}^{2} T, k\right) \\
+ & \frac{C_{4}\left(a ; x_{\mathrm{av}}\right)_{0}^{T}+C_{4}\left(\widetilde{a} ; x_{\mathrm{av}}\right)_{0}^{T}}{2}\left(3 \partial_{x^{4}}^{4}-6 \partial_{x^{3}}^{3}+\frac{7}{2} \partial_{x^{2}}^{2}-\frac{1}{2} \partial_{x}\right) \mathrm{Call}^{\mathrm{BS}}\left(x_{0}, \bar{a}_{x_{\mathrm{av}}}^{2} T, k\right)
\end{aligned}
$$




$$
\begin{aligned}
& +\frac{\left[C_{1}\left(a ; x_{\mathrm{av}}\right)_{0}^{T}\right]^{2}+\left[C_{1}\left(\widetilde{a} ; x_{\mathrm{av}}\right)_{0}^{T}\right]^{2}}{2} \\
& \quad \times\left(\frac{1}{2} \partial_{x^{6}}^{6}-\frac{3}{2} \partial_{x^{5}}^{5}+\frac{13}{8} \partial_{x^{4}}^{4}-\frac{3}{4} \partial_{x^{3}}^{3}+\frac{1}{8} \partial_{x^{2}}^{2}\right) \mathrm{Call}^{\mathrm{BS}}\left(x_{0}, \bar{a}_{x_{\mathrm{av}}}^{2} T, k\right) \\
& +\mathcal{O}\left(\mathcal{M}_{1}(a)\left[\mathcal{M}_{0}(a)\right]^{3} T^{2}\right) .
\end{aligned}
$$

Secondly, we pass to the corrective terms in which appears the operator $C_{1}$. For the sake of clarity, we introduce the following notation $\mathcal{A}_{x}=\partial_{x^{3}}^{3}-\frac{3}{2} \partial_{x^{2}}^{2}+\frac{1}{2} \partial_{x}$ and $\mathcal{A}_{z}=\partial_{z^{3}}^{3}-\frac{3}{2} \partial_{z^{2}}^{2}+\frac{1}{2} \partial_{z}$. For example $\partial_{y} \mathcal{A}_{x}$ stands for the differential operator $\partial_{y x^{3}}^{4}-\frac{3}{2} \partial_{y x^{2}}^{3}+\frac{1}{2} \partial_{y x}^{2}$ and similarly for $\partial_{y^{2}}^{2} \mathcal{A}_{x}$. We recall the following relation $\mathcal{A}_{x} \mathrm{Call}^{\mathrm{BS}}=-\mathcal{A}_{z} \mathrm{Call}^{\mathrm{BS}}$ (see (72) in Proposition 34). Our purpose is to prove that:

$$
\begin{aligned}
& \frac{1}{2} C_{1}\left(a ; x_{0}\right)_{0}^{T} \mathcal{A}_{x} \operatorname{Call}^{\mathrm{BS}}\left(x_{0}, \bar{a}_{x_{0}}^{2} T, k\right)+\frac{1}{2} C_{1}(\widetilde{a} ; k)_{0}^{T} \mathcal{A}_{z} \operatorname{Call}^{\mathrm{BS}}\left(x_{0}, \bar{a}_{k}^{2} T, k\right) \\
= & \frac{1}{2}\left(C_{1}\left(a ; x_{\mathrm{av}}\right)_{0}^{T}-C_{1}\left(\widetilde{a} ; x_{\mathrm{av}}\right)_{0}^{T}\right) \mathcal{A}_{x} \operatorname{Call}^{\mathrm{BS}}\left(x_{0}, \bar{a}_{x_{\mathrm{av}}}^{2} T, k\right) \\
& +\frac{\left(x_{0}-k\right)}{4}\left[4 C_{6}\left(a ; x_{\mathrm{av}}\right)_{0}^{T}+C_{2}\left(a ; x_{\mathrm{av}}\right)_{0}^{T}+C_{2}\left(\widetilde{a} ; x_{\mathrm{av}}\right)_{0}^{T}\right] \mathcal{A}_{x} \mathrm{Call}^{\mathrm{BS}}\left(x_{0}, \bar{a}_{x_{\mathrm{av}}}^{2} T, k\right) \\
& +\left[C_{4}\left(a ; x_{\mathrm{av}}\right)_{0}^{T}+C_{4}\left(\widetilde{a} ; x_{\mathrm{av}}\right)_{0}^{T}+\omega\left(a\left(x_{\mathrm{av}}\right) a^{(1)}\left(x_{\mathrm{av}}\right), a^{2}\left(x_{\mathrm{av}}\right), a\left(x_{\mathrm{av}}\right) a^{(1)}\left(x_{\mathrm{av}}\right)\right)_{0}^{T}\right] \\
& \quad \times\left(x_{0}-k\right) \partial_{y} \mathcal{A}_{x} \operatorname{Call}^{\mathrm{BS}}\left(x_{0}, \bar{a}_{x_{\mathrm{av}}}^{2} T, k\right)+\mathcal{O}\left(\mathcal{M}_{1}(a)\left[\mathcal{M}_{0}(a)\right]^{3} T^{2}\right) .
\end{aligned}
$$

Perform a second order Taylor expansion for the function $y \rightarrow \mathcal{A}_{x} \mathrm{Call}^{\mathrm{BS}}\left(x_{0}, y, k\right)$ at $y=\bar{a}_{x_{0}}^{2} T=\omega\left(a^{2}\left(x_{0}\right)\right)_{0}^{T}$ around $y=\bar{a}_{x_{\mathrm{av}}}^{2} T=\omega\left(a^{2}\left(x_{\mathrm{av}}\right)\right)_{0}^{T}$ and for the function $x \rightarrow C_{1}(a ; x)_{0}^{T}$ at $x=x_{0}$ around $x=x_{\mathrm{av}}$ :

$$
\begin{aligned}
& C_{1}\left(a ; x_{0}\right)_{0}^{T} \mathcal{A}_{x} \operatorname{Call}^{\mathrm{BS}}\left(x_{0}, \bar{a}_{x_{0}}^{2} T, k\right) \\
= & \left\{C_{1}\left(a ; x_{\mathrm{av}}\right)_{0}^{T}+\left.\partial_{x}\left(C_{1}(a ; x)_{0}^{T}\right)\right|_{x=x_{\mathrm{av}}} \frac{\left(x_{0}-k\right)}{2}+R_{1}\right\} \\
& \times\left\{\mathcal{A}_{x} \mathrm{Call}^{\mathrm{BS}}\left(x_{0}, \bar{a}_{x_{\mathrm{av}}}^{2} T, k\right)+\partial_{y} \mathcal{A}_{x} \mathrm{Call}^{\mathrm{BS}}\left(x_{0}, \bar{a}_{x_{\mathrm{av}}}^{2} T, k\right) T\left(\bar{a}_{x_{0}}^{2}-\bar{a}_{x_{\mathrm{av}}}^{2}\right)+R_{2}\right\} \\
= & \left\{C_{1}\left(a ; x_{\mathrm{av}}\right)_{0}^{T}+\left.\partial_{x}\left(C_{1}(a ; x)_{0}^{T}\right)\right|_{x=x_{\mathrm{av}}} \frac{\left(x_{0}-k\right)}{2}+R_{1}\right\} \\
& \times\left\{\mathcal{A}_{x} \mathrm{Call}^{\mathrm{BS}}\left(x_{0}, \bar{a}_{x_{\mathrm{av}}}^{2} T, k\right)+R_{3}+R_{2}\right. \\
& \left.+\partial_{y} \mathcal{A}_{x} \mathrm{Call}^{\mathrm{BS}}\left(x_{0}, \bar{a}_{x_{\mathrm{av}}}^{2} T, k\right) \omega\left(a\left(x_{\mathrm{av}}\right) a^{(1)}\left(x_{\mathrm{av}}\right)\right)_{0}^{T}\left(x_{0}-k\right)\right\} \\
= & C_{1}\left(a ; x_{\mathrm{av}}\right)_{0}^{T} \mathcal{A}_{x} \mathrm{Call}^{\mathrm{BS}}\left(x_{0}, \bar{a}_{x_{\mathrm{av}}}^{2} T, k\right) \\
& +\left.\partial_{x}\left(C_{1}(a ; x)_{0}^{T}\right)\right|_{x=x_{\mathrm{av}}} \frac{\left(x_{0}-k\right)}{2} \mathcal{A}_{x} \mathrm{Call}^{\mathrm{BS}}\left(x_{0}, \bar{a}_{x_{\mathrm{av}}}^{2} T, k\right) \\
& +C_{1}\left(a ; x_{\mathrm{av}}\right)_{0}^{T} \partial_{y} \mathcal{A}_{x} \mathrm{Call}^{\mathrm{BS}}\left(x_{0}, \bar{a}_{x_{\mathrm{av}}}^{2} T, k\right) \omega\left(a\left(x_{\mathrm{av}}\right) a^{(1)}\left(x_{\mathrm{av}}\right)\right)_{0}^{T}\left(x_{0}-k\right) \\
& +R,
\end{aligned}
$$


where:

$$
\begin{aligned}
R= & C_{1}\left(a ; x_{0}\right)_{0}^{T}\left[R_{3}+R_{2}\right]+R_{1} \mathcal{A}_{x} \mathrm{Call}^{\mathrm{BS}}\left(x_{0}, \bar{a}_{x_{\mathrm{av}}}^{2} T, k\right) \\
& +\left(x_{0}-k\right) \partial_{y} \mathcal{A}_{x} \mathrm{Call}^{\mathrm{BS}}\left(x_{0}, \bar{a}_{x_{\mathrm{av}}}^{2} T, k\right) \omega\left(a\left(x_{\mathrm{av}}\right) a^{(1)}\left(x_{\mathrm{av}}\right)\right)_{0}^{T} \\
& \times\left[R_{1}+\left.\partial_{x}\left(\left.C_{1}(a ; x)\right|_{0} ^{T}\right)\right|_{x=x_{\mathrm{av}}} \frac{\left(x_{0}-k\right)}{2}\right], \\
R_{1}= & \left.\frac{\left(x_{0}-k\right)^{2}}{4} \int_{0}^{1}\left(\partial_{x^{2}}^{2}\left(C_{1}(a ; x)_{0}^{T}\right)\right)\right|_{x=\lambda x_{0}+(1-\lambda) x_{\mathrm{av}}}(1-\lambda) \mathrm{d} \lambda, \\
R_{2}= & \left.T^{2}\left(\bar{a}_{x_{0}}^{2}-\bar{a}_{x_{\mathrm{av}}}\right)^{2} \int_{0}^{1}\left(\partial_{y^{2}}^{2} \mathcal{A}_{x} \mathrm{Call}^{\mathrm{BS}}\left(x_{0}, y, k\right)\right)\right|_{y=T\left(\lambda \bar{a}_{x_{0}}^{2}+(1-\lambda) \bar{a}_{x_{\mathrm{av}}}\right)}(1-\lambda) \mathrm{d} \lambda, \\
R_{3}= & \partial_{y} \mathcal{A}_{x} \operatorname{Call}^{\mathrm{BS}}\left(x_{0}, \bar{a}_{x_{\mathrm{av}}}^{2} T, k\right) \frac{\left(x_{0}-k\right)^{2}}{4} \\
& \times\left.\int_{0}^{1}\left(\partial_{x^{2}}^{2}\left(\omega\left(a^{2}(x)\right)_{0}^{T}\right)\right)\right|_{x=\lambda x_{0}+(1-\lambda) x_{\mathrm{av}}}(1-\lambda) \mathrm{d} \lambda .
\end{aligned}
$$

On the one hand, we have:

$$
\begin{aligned}
& \partial_{z}\left(C_{1}(l ; z)_{0}^{T}\right)=2 C_{6}(l ; z)_{0}^{T}+C_{2}(l ; z)_{0}^{T}, \\
& C_{1}(l ; z)_{0}^{T} \omega\left(l(z) l^{(1)}(z)\right)_{0}^{T}=2 C_{4}(l ; z)_{0}^{T}+\omega\left(l(z) l^{(1)}(z), l^{2}(z), l(z) l^{(1)}(z)\right)_{0}^{T},
\end{aligned}
$$

and on the other hand, with (6), Corollary 30 and $\left(\mathcal{H}^{a}\right)$, it comes:

$$
|R| \leq_{c} \mathcal{M}_{1}(a)\left[\mathcal{M}_{0}(a)\right]^{3} T^{2} .
$$

We skip further details. Consequently we can write (52) as follows:

$$
\begin{aligned}
& C_{1}\left(a ; x_{0}\right)_{0}^{T} \mathcal{A}_{x} \operatorname{Call}^{\mathrm{BS}}\left(x_{0}, \bar{a}_{x_{0}}^{2} T, k\right) \\
& =C_{1}\left(a ; x_{\mathrm{av}}\right)_{0}^{T} \mathcal{A}_{x} \operatorname{Call}^{\mathrm{BS}}\left(x_{0}, \bar{a}_{x_{\mathrm{av}}}^{2} T, k\right) \\
& +\frac{\left(x_{0}-k\right)}{2}\left[2 C_{6}\left(a ; x_{\mathrm{av}}\right)_{0}^{T}+C_{2}\left(a ; x_{\mathrm{av}}\right)_{0}^{T}\right] \mathcal{A}_{x} \operatorname{Call}^{\mathrm{BS}}\left(x_{0}, \bar{a}_{x_{\mathrm{av}}}^{2} T, k\right) \\
& +\left[2 C_{4}\left(a ; x_{\mathrm{av}}\right)_{0}^{T}+\omega\left(a\left(x_{\mathrm{av}}\right) a^{(1)}\left(x_{\mathrm{av}}\right), a^{2}\left(x_{\mathrm{av}}\right), a\left(x_{\mathrm{av}}\right) a^{(1)}\left(x_{\mathrm{av}}\right)\right)_{0}^{T}\right]\left(x_{0}-k\right) \\
& \quad \quad \times \partial_{y} \mathcal{A}_{x} \operatorname{Call}^{\mathrm{BS}}\left(x_{0}, \bar{a}_{x_{\mathrm{av}}}^{2} T, k\right)+\mathcal{O}\left(\mathcal{M}_{1}(a)\left[\mathcal{M}_{0}(a)\right]^{3} T^{2}\right) .
\end{aligned}
$$

Then using the relation $\mathcal{A}_{x} \mathrm{Call}^{\mathrm{BS}}=-\mathcal{A}_{z} \mathrm{Call}^{\mathrm{BS}}$, the time reversal invariance of $l \rightarrow C_{6}(l, z)_{0}^{T}$ and $l \rightarrow \omega\left(l(z) l^{(1)}(z), l^{2}(z), l(z) l^{(1)}(z)\right)_{0}^{T}$ (for any $z$ ), one obtains similarly:

$$
\begin{aligned}
C_{1}(\widetilde{a} ; k)_{0}^{T} \mathcal{A}_{z} \operatorname{Call}^{\mathrm{BS}}\left(x_{0}, \bar{a}_{x_{0}}^{2} T, k\right) \\
=-C_{1}\left(\widetilde{a} ; x_{\mathrm{av}}\right)_{0}^{T} \mathcal{A}_{x} \operatorname{Call}^{\mathrm{BS}}\left(x_{0}, \bar{a}_{x_{\mathrm{av}}}^{2} T, k\right) \\
+\frac{\left(x_{0}-k\right)}{2}\left[2 C_{6}\left(a ; x_{\mathrm{av}}\right)_{0}^{T}+C_{2}\left(\widetilde{a} ; x_{\mathrm{av}}\right)_{0}^{T}\right] \mathcal{A}_{x} \operatorname{Call}^{\mathrm{BS}}\left(x_{0}, \bar{a}_{x_{\mathrm{av}}}^{2} T, k\right) \\
+\left[2 C_{4}\left(\widetilde{a} ; x_{\mathrm{av}}\right)_{0}^{T}+\omega\left(a\left(x_{\mathrm{av}}\right) a^{(1)}\left(x_{\mathrm{av}}\right), a^{2}\left(x_{\mathrm{av}}\right), a\left(x_{\mathrm{av}}\right) a^{(1)}\left(x_{\mathrm{av}}\right)\right)_{0}^{T}\right]\left(x_{0}-k\right) \\
\quad \times \partial_{y} \mathcal{A}_{x} \operatorname{Call}^{\mathrm{BS}}\left(x_{0}, \bar{a}_{x_{\mathrm{av}}}^{2} T, k\right)+\mathcal{O}\left(\mathcal{M}_{1}(a)\left[\mathcal{M}_{0}(a)\right]^{3} T^{2}\right) .
\end{aligned}
$$

Compute the average of (53) and (54) to complete the proof of (51). 
$\triangleright$ Step 3: mathematical reductions. We gather terms coming from (48) and (51). In view of (6) and equations (71) and (72) in Proposition 34, we have:

$$
\begin{aligned}
& \frac{\left(x_{0}-k\right)^{2}}{4} C_{5}\left(a ; x_{\mathrm{av}}\right)_{0}^{T} \partial_{y} \operatorname{Call}^{\mathrm{BS}}\left(x_{0}, \bar{a}_{x_{\mathrm{av}}}^{2} T, k\right) \\
& +\frac{\left(x_{0}-k\right)}{4}\left[C_{2}\left(a ; x_{\mathrm{av}}\right)_{0}^{T}+C_{2}\left(\widetilde{a} ; x_{\mathrm{av}}\right)_{0}^{T}\right] \mathcal{A}_{x} \operatorname{Call}^{\mathrm{BS}}\left(x_{0}, \bar{a}_{x_{\mathrm{av}}}^{2} T, k\right) \\
= & \frac{\left(x_{0}-k\right)^{2}}{4} \partial_{y} \operatorname{Call}^{\mathrm{BS}}\left(x_{0}, \bar{a}_{x_{\mathrm{av}}}^{2} T, k\right)\left(C_{5}\left(a ; x_{\mathrm{av}}\right)_{0}^{T}-2 \frac{\left[C_{2}\left(a ; x_{\mathrm{av}}\right)_{0}^{T}+C_{2}\left(\widetilde{a} ; x_{\mathrm{av}}\right)_{0}^{T}\right]}{\omega\left(a^{2}\left(x_{\mathrm{av}}\right)\right)_{0}^{T}}\right) \\
= & \frac{\left(x_{0}-k\right)^{2}}{4} \partial_{y} \operatorname{Call}^{\mathrm{BS}}\left(x_{0}, \bar{a}_{x_{\mathrm{av}}}^{2} T, k\right)\left(C_{5}\left(a ; x_{\mathrm{av}}\right)_{0}^{T}-2 C_{5}\left(a ; x_{\mathrm{av}}\right)_{0}^{T}\right) \\
= & -\frac{\left(x_{0}-k\right)^{2}}{8} C_{5}\left(a ; x_{\mathrm{av}}\right)_{0}^{T}\left(\partial_{x^{2}}^{2}-\partial_{x}\right) \operatorname{Call}^{\mathrm{BS}}\left(x_{0}, \bar{a}_{x_{\mathrm{av}}}^{2} T, k\right),
\end{aligned}
$$

where we have used at the second equality the relation $C_{5}(l ; z)_{0}^{T} \omega\left(l^{2}(z)\right)_{0}^{T}=$ $C_{2}(l ; z)_{0}^{T}+C_{2}(\widetilde{l} ; z)_{0}^{T}$ obtained easily with (26). Then (6), (71) and (72) yield

$$
\begin{aligned}
& \partial_{y} \mathcal{A}_{x} \operatorname{Call}^{\mathrm{BS}}\left(x_{0}, \bar{a}_{x_{\mathrm{av}}}^{2} T, k\right) \\
= & \left.\partial_{y}\left(\left(-\frac{2\left(x_{0}-k\right)}{y} \partial_{y}\right) \operatorname{Call}^{\mathrm{BS}}\left(x_{0}, y, k\right)\right)\right|_{y=\bar{a}_{x_{\mathrm{av}}}^{2} T} \\
= & \frac{2\left(x_{0}-k\right)}{\omega\left(a^{2}\left(x_{\mathrm{av}}\right)\right)_{0}^{T}}\left[\frac{\partial_{y} \operatorname{Call}^{\mathrm{BS}}\left(x_{0}, \bar{a}_{x_{\mathrm{a}}}^{2} T, k\right)}{\omega\left(a^{2}\left(x_{\mathrm{av}}\right)\right)_{0}^{T}}-\partial_{y^{2}}^{2} \operatorname{Call}^{\mathrm{BS}}\left(x_{0}, \bar{a}_{x_{\mathrm{av}}}^{2} T, k\right)\right],
\end{aligned}
$$

and straightforward calculus allows to obtain with (26):

$$
C_{6}(l ; z)_{0}^{T} \omega\left(l^{2}(z)\right)_{0}^{T}=C_{4}(l ; z)_{0}^{T}+C_{4}(\widetilde{l} ; z)_{0}^{T}+\omega\left(l(z) l^{(1)}(z), l^{2}(z), l(z) l^{(1)}(z)\right)_{0}^{T} .
$$

These two intermediate results give:

$$
\begin{aligned}
& \left(x_{0}-k\right) C_{6}\left(a ; x_{\mathrm{av}}\right)_{0}^{T}\left[\mathcal{A}_{x} \mathrm{Call}^{\mathrm{BS}}\left(x_{0}, \bar{a}_{x_{\mathrm{av}}}^{2} T, k\right)+\left(x_{0}-k\right) \partial_{y^{2}}^{2} \operatorname{Call}^{\mathrm{BS}}\left(x_{0}, \bar{a}_{x_{\mathrm{av}}}^{2} T, k\right)\right] \\
+ & {\left[C_{4}\left(a ; x_{\mathrm{av}}\right)_{0}^{T}+C_{4}\left(\widetilde{a} ; x_{\mathrm{av}}\right)_{0}^{T}+\omega\left(a\left(x_{\mathrm{av}}\right) a^{(1)}\left(x_{\mathrm{av}}\right), a^{2}\left(x_{\mathrm{av}}\right), a\left(x_{\mathrm{av}}\right) a^{(1)}\left(x_{\mathrm{av}}\right)\right)_{0}^{T}\right] } \\
& \times\left(x_{0}-k\right) \partial_{y} \mathcal{A}_{x} \mathrm{Call}^{\mathrm{BS}}\left(x_{0}, \omega\left(a^{2}\left(x_{\mathrm{av}}\right)\right)_{0}^{T}, k\right) \\
=- & 2 \frac{\left(x_{0}-k\right)^{2}}{\omega\left(a^{2}\left(x_{\mathrm{av}}\right)\right)_{0}^{T}} C_{6}\left(a ; x_{\mathrm{av}}\right)_{0}^{T} \partial_{y} \operatorname{Call}^{\mathrm{BS}}\left(x_{0}, \bar{a}_{x_{\mathrm{av}}}^{2} T, k\right) \\
+ & \left(x_{0}-k\right)^{2} C_{6}\left(a ; x_{\mathrm{av}}\right)_{0}^{T} \partial_{y^{2}}^{2} \mathrm{Call}^{\mathrm{BS}}\left(x_{0}, \bar{a}_{x_{\mathrm{av}}}^{T} T, k\right) \\
+ & \left(x_{0}-k\right)\left[C_{6}\left(a ; x_{\mathrm{av}}\right)_{0}^{T} \omega\left(a^{2}\left(x_{\mathrm{av}}\right)\right)_{0}^{T}\right] \frac{2\left(x_{0}-k\right)}{\omega\left(a^{2}\left(x_{\mathrm{av}}\right)\right)_{0}^{T}} \\
& \times\left[\frac{\partial_{y} \mathrm{Call}{ }^{\mathrm{BS}}\left(x_{0}, \omega\left(a^{2}\left(x_{\mathrm{av}}\right)\right)_{0}^{T}, k\right)}{\omega\left(a^{2}\left(x_{\mathrm{av}}\right)\right)_{0}^{T}}-\partial_{y^{2}}^{2} \mathrm{Call}^{\mathrm{BS}}\left(x_{0}, \omega\left(a^{2}\left(x_{\mathrm{av}}\right)\right)_{0}^{T}, k\right)\right] \\
=- & \left(x_{0}-k\right)^{2} C_{6}\left(a ; x_{\mathrm{av}}\right)_{0}^{T} \partial_{y^{2}}^{2} \mathrm{Call}^{\mathrm{BS}}\left(x_{0}, \bar{a}_{x_{\mathrm{av}}}^{2} T, k\right) \\
=- & \frac{\left(x_{0}-k\right)^{2}}{4} C_{6}\left(a ; x_{\mathrm{av}}\right)_{0}^{T}\left(\partial_{x^{4}}^{4}-2 \partial_{x^{3}}^{3}+\partial_{x^{2}}^{2}\right) \mathrm{Call}^{\mathrm{BS}}\left(x_{0}, \bar{a}_{x_{\mathrm{av}}}^{2} T, k\right) .
\end{aligned}
$$

Finally, sum the relations (48-51-50) taking into account the simplifications (55-56) and apply Theorems 18 and 19 to obtain the announced result (47). 


\subsection{Third order expansion of the implied volatility}

We define extra integral operators in order to state a new result about third order expansions of the implied volatility.

Definition 21. Provided that the derivatives and the integrals below have a meaning, we define the following operators for a two variables non-negative function $l$ such that $\bar{l}_{z}>0$ :

$$
\begin{gathered}
\gamma_{0}(l ; z)_{0}^{T}=\bar{l}_{z}+\frac{C_{2}(l ; z)_{0}^{T}}{2 \bar{l}_{z} T}-\frac{C_{4}(l ; z)_{0}^{T}}{4 \bar{l}_{z} T}-\frac{C_{3}(l ; z)_{0}^{T}}{\bar{l}_{z}^{3} T^{2}}-\frac{3 C_{4}(l ; z)_{0}^{T}}{\bar{l}_{z}^{3} T^{2}}+\frac{\left[C_{1}(l ; z)_{0}^{T}\right]^{2}}{8 \bar{l}_{z}^{3} T^{2}} \\
\quad+\frac{3\left[C_{1}(l ; z)_{0}^{T}\right]^{2}}{2 \bar{l}_{z}^{5} T^{3}}, \\
\gamma_{1}(l ; z)_{0}^{T}=\frac{C_{1}(l ; z)_{0}^{T}}{\bar{l}_{z}^{3} T^{2}}, \quad \gamma_{2}(l ; z)_{0}^{T}=\frac{C_{3}(l ; z)_{0}^{T}}{\bar{l}_{z}^{5} T^{3}}+3 \frac{C_{4}(l ; z)_{0}^{T}}{\bar{l}_{z}^{5} T^{3}}-\frac{3\left[C_{1}(l ; z)_{0}^{T}\right]^{2}}{\bar{l}_{z}^{7} T^{4}} ; \\
\pi_{0}(l ; z)_{0}^{T}=\frac{\gamma_{0}(l ; z)_{0}^{T}+\gamma_{0}(\widetilde{l} ; z)_{0}^{T}}{2}, \quad \pi_{1}(l ; z)_{0}^{T}=\frac{\gamma_{1}(\widetilde{l} ; z)_{0}^{T}-\gamma_{1}(l ; z)_{0}^{T}}{2}, \\
\pi_{2}(l ; z)_{0}^{T}=\frac{\gamma_{2}(l ; z)_{0}^{T}+\gamma_{2}(\widetilde{l} ; z)_{0}^{T}}{2}-\frac{C_{5}(l ; z)_{0}^{T}}{8 \bar{l}_{z} T}+\frac{C_{6}(l ; z)_{0}^{T}}{4 \bar{l}_{z}^{3} T^{2}} ; \\
\chi_{0}(l ; z)_{0}^{T}=\bar{l}_{z}+\frac{C_{2}(l ; z)_{0}^{T}}{2 \bar{l}_{z} T}-\frac{C_{3}(l ; z)_{0}^{T}}{\bar{l}_{z}^{3} T^{2}}-\frac{3 C_{4}(l ; z)_{0}^{T}}{\bar{l}_{z}^{3} T^{2}}+\frac{3\left[C_{1}(l ; z)_{0}^{T}\right]^{2}}{2 \bar{l}_{z}^{5} T^{3}}, \\
\chi_{1}(l ; z)_{0}^{T}=\gamma_{1}(l ; z)_{0}^{T}, \quad \chi_{2}(l ; z)_{0}^{T}=\gamma_{2}(l ; z)_{0}^{T} ; \\
\Xi_{0}(l ; z)_{0}^{T}=\frac{\chi_{0}(l ; z)_{0}^{T}+\chi_{0}(\widetilde{l} ; z)_{0}^{T}}{2}, \quad \Xi_{1}(l ; z)_{0}^{T}=\pi_{1}(l ; z)_{0}^{T}, \quad \Xi_{2}(l ; z)_{0}^{T}=\pi_{2}(l ; z)_{0}^{T} .
\end{gathered}
$$

Theorem 22. (3rd order expansions of the implied volatility). Assume $\left(\mathcal{H}^{a}\right)$. We have:

$$
\begin{aligned}
& \sigma_{\mathrm{I}}\left(x_{0}, T, k\right)=\gamma_{0}\left(a ; x_{0}\right)_{0}^{T}-\gamma_{1}\left(a ; x_{0}\right)_{0}^{T} m+\gamma_{2}\left(a ; x_{0}\right)_{0}^{T} m^{2}+\operatorname{Error}_{3, \mathrm{x}_{0}}^{\mathrm{I}}, \\
& \sigma_{\mathrm{I}}\left(x_{0}, T, k\right)=\gamma_{0}(\widetilde{a} ; k)_{0}^{T}+\gamma_{1}(\widetilde{a} ; k)_{0}^{T} m+\gamma_{2}(\widetilde{a} ; k)_{0}^{T} m^{2}+\text { Error }_{3, \mathrm{k}}^{\mathrm{I}}, \\
& \sigma_{\mathrm{I}}\left(x_{0}, T, k\right)=\pi_{0}\left(a ; x_{\mathrm{av}}\right)_{0}^{T}+\pi_{1}\left(a ; x_{\mathrm{av}}\right)_{0}^{T} m+\pi_{2}\left(a ; x_{\mathrm{av}}\right)_{0}^{T} m^{2}+\text { Error }_{3, \mathrm{x}_{\mathrm{av}}}^{\mathrm{I}} .
\end{aligned}
$$

Under $\left(\mathcal{H}^{\Sigma}\right)$ we have

$$
\begin{aligned}
& \Sigma_{\mathrm{I}}\left(S_{0}, T, K\right)=\chi_{0}\left(\Sigma ; S_{0}\right)_{0}^{T}-\chi_{1}\left(\Sigma ; S_{0}\right)_{0}^{T} M+\chi_{2}\left(\Sigma ; S_{0}\right)_{0}^{T} M^{2}+\text { Error }_{3, \mathrm{~S}_{0}}^{\mathrm{I}}, \\
& \Sigma_{\mathrm{I}}\left(S_{0}, T, K\right)=\chi_{0}(\widetilde{\Sigma} ; K)_{0}^{T}+\chi_{1}(\widetilde{\Sigma} ; K)_{0}^{T} M+\chi_{2}(\widetilde{\Sigma} ; K)_{0}^{T} M^{2}+\text { Error }_{3, \mathrm{~K}}^{\mathrm{I}}, \\
& \Sigma_{\mathrm{I}}\left(S_{0}, T, K\right)=\Xi_{0}\left(\Sigma ; S_{\mathrm{av}}\right)_{0}^{T}+\Xi_{1}\left(\Sigma ; S_{\mathrm{av}}\right)_{0}^{T} M+\Xi_{2}\left(\Sigma ; S_{\mathrm{av}}\right)_{0}^{T} M^{2}+\text { Error }_{3, \mathrm{~S}_{\mathrm{av}}}^{\mathrm{I}} .
\end{aligned}
$$

The operators $\gamma_{i}, \pi_{i}, \chi_{i}$ and $\Xi_{i}$ used in the above expansions are defined in Definition 21.

Remark 23. We have obtained Black-Scholes (respectively Bachelier) implied volatility approximations which are written as a quadratic function w.r.t. the BlackScholes log-moneyness (respectively w.r.t. the Bachelier moneyness). At the money, 
observe that the corresponding approximations are not equal to the local volatility function computed at spot. However, in view of the definition of the operators $C_{1}$, $C_{2}, C_{3}$ and $C_{4}$ (see Definition 17) and the operators $\gamma_{0}$ and $\chi_{0}$ (see Definition 21), we easily obtain the estimate:

$\left|\gamma_{0}\left(a ; x_{0}\right)_{0}^{T}-\bar{a}_{x_{0}}\right|+\left|\gamma_{0}\left(\widetilde{a} ; x_{0}\right)_{0}^{T}-\bar{a}_{x_{0}}\right|+\left|\chi_{0}\left(\Sigma ; S_{0}\right)-\bar{\Sigma}_{S_{0}}\right|+\left|\chi_{0}\left(\widetilde{\Sigma} ; S_{0}\right)-\bar{\Sigma}_{S_{0}}\right| \leq_{c} T$.

It shows that when the maturity tends to zero, our implied volatility approximations at the money become equal to the local volatility function frozen at spot. We therefore interpret the difference between our implied volatility approximations ATM and the local volatility function frozen at spot as a maturity bias.

Proof. We first focus on the formula (57), the treatment of (58-60-61) being similar. Start from Theorem 18 and apply Proposition 34 in order to write the Greeks w.r.t. $x$ (for each operator $C_{i}$ ) in terms of the Vega ${ }^{\mathrm{BS}}$ and the Vomma ${ }^{\mathrm{BS}}$. Thus the third order expansion formula based on the ATM local volatility with log-normal proxy can be transformed into:

$$
\begin{aligned}
& \operatorname{Call}\left(e^{x_{0}}, T, e^{k}\right) \\
= & \operatorname{Call}^{\mathrm{BS}}\left(x_{0}, \bar{a}_{x_{0}}^{2} T, k\right)+\operatorname{Vega}^{\mathrm{BS}}\left(x_{0}, \bar{a}_{x_{0}}^{2} T, k\right) \\
\times & {\left[-\frac{C_{1}\left(a ; x_{0}\right)_{0}^{T} m}{\bar{a}_{x_{0}}^{3} T^{2}}+\frac{C_{2}\left(a ; x_{0}\right)_{0}^{T}}{2 \bar{a}_{x_{0}} T}+\frac{C_{3}\left(a ; x_{0}\right)_{0}^{T} m^{2}}{\bar{a}_{x_{0}}^{5} T^{3}}-\frac{C_{3}\left(a ; x_{0}\right)_{0}^{T}}{\bar{a}_{x_{0}}^{3} T^{2}}+\frac{3 C_{4}\left(a ; x_{0}\right)_{0}^{T} m^{2}}{\bar{a}_{x_{0}}^{5} T^{3}}\right.} \\
- & \left.\frac{3 C_{4}\left(a ; x_{0}\right)_{0}^{T}}{\bar{a}_{x_{0}}^{3} T^{2}}-\frac{C_{4}\left(a ; x_{0}\right)_{0}^{T}}{4 \bar{a}_{x_{0}} T}+\frac{\left[C_{1}\left(a ; x_{0}\right)_{0}^{T}\right]^{2}}{8 \bar{a}_{x_{0}}^{3} T^{2}}+\frac{3\left[C_{1}\left(a ; x_{0}\right)_{0}^{T}\right]^{2}}{2 \bar{a}_{x_{0}}^{5} T^{3}}-\frac{3\left[C_{1}\left(a ; x_{0}\right)_{0}^{T}\right]^{2} m^{2}}{\bar{a}_{x_{0}}^{7} T^{4}}\right] \\
& +\frac{1}{2} \operatorname{Vomma}^{\mathrm{BS}}\left(x_{0}, \bar{a}_{x_{0}}^{2} T, k\right)\left(\frac{C_{1}\left(a ; x_{0}\right)_{0}^{T} m}{\bar{a}_{x_{0}}^{3} T^{2}}\right)^{2}+\mathcal{O}\left(\mathcal{M}_{1}(a)\left[\mathcal{M}_{0}(a)\right]^{3} T^{2}\right) \\
= & \operatorname{Call}^{\mathrm{BS}}\left(x_{0}, \bar{a}_{x_{0}}^{2} T, k\right) \\
& +\operatorname{Vega}^{\mathrm{BS}}\left(x_{0}, \bar{a}_{x_{0}}^{2} T, k\right)\left[\gamma_{0}\left(a ; x_{0}\right)_{0}^{T}-\bar{a}_{x_{0}}-\gamma_{1}\left(a ; x_{0}\right)_{0}^{T} m+\gamma_{2}\left(a ; x_{0}\right)_{0}^{T} m^{2}\right] \\
& +\frac{1}{2} \operatorname{Vomma}^{\mathrm{BS}}\left(x_{0}, \bar{a}_{x_{0}}^{2} T, k\right)\left[\gamma_{1}\left(a ; x_{0}\right)_{0}^{T} m\right]^{2}+\mathcal{O}\left(\mathcal{M}_{1}(a)\left[\mathcal{M}_{0}(a)\right]^{3} T^{2}\right) \\
\approx & \operatorname{Call}^{\mathrm{BS}}\left(x_{0},\left[\gamma_{0}\left(a ; x_{0}\right)_{0}^{T}-\gamma_{1}\left(a ; x_{0}\right)_{0}^{T} m+\gamma_{2}\left(a ; x_{0}\right)_{0}^{T} m^{2}\right]^{2} T, k\right) .
\end{aligned}
$$

This reads as an expansion of the implied volatility and achieves the proof of (57).

Now we give the main lines of the derivation of the error estimate in (59), while (62) is left to the reader. Again, we apply Theorem 20 and Proposition 34 in order 
to replace the $x_{0}$-Greeks with the Vega ${ }^{\mathrm{BS}}$ and the Vomma ${ }^{\mathrm{BS}}$. One obtains similarly:

$$
\begin{aligned}
\operatorname{Call}( & \left(e^{x_{0}}, T, e^{k}\right)=\operatorname{Call}^{\mathrm{BS}}\left(x_{0}, \bar{a}_{x_{\mathrm{av}}}^{2} T, k\right) \\
& +\operatorname{Vega}^{\mathrm{BS}}\left(x_{0}, \bar{a}_{x_{\mathrm{av}}}^{2} T, k\right)\left[\frac{\gamma_{0}\left(a ; x_{\mathrm{av}}\right)_{0}^{T}+\gamma_{0}\left(\widetilde{a} ; x_{\mathrm{av}}\right)_{0}^{T}}{2}-\bar{a}_{x_{\mathrm{av}}}\right. \\
& +\frac{\gamma_{1}\left(\widetilde{a} ; x_{\mathrm{av}}\right)_{0}^{T}-\gamma_{1}\left(a ; x_{\mathrm{av}}\right)_{0}^{T}}{2} m+\frac{\gamma_{2}\left(a ; x_{\mathrm{av}}\right)_{0}^{T}+\gamma_{2}\left(\widetilde{a} ; x_{\mathrm{av}}\right)_{0}^{T}}{2} m^{2} \\
& \left.-\frac{C_{5}\left(a ; x_{\mathrm{av}}\right)_{0}^{T}}{8 \bar{a}_{x_{\mathrm{av}}} T} m^{2}+\frac{C_{6}\left(a ; x_{\mathrm{av}}\right)_{0}^{T}}{4 \bar{a}_{x_{\mathrm{av}}}^{3} T^{2}} m^{2}+\frac{C_{6}\left(a ; x_{\mathrm{av}}\right)_{0}^{T}}{16 \bar{a}_{x_{\mathrm{av}}} T} m^{2}-\frac{C_{6}\left(a ; x_{\mathrm{av}}\right)_{0}^{T}}{4 \bar{a}_{x_{\mathrm{av}}}^{5} T^{3}} m^{4}\right] \\
& +\frac{1}{2} \operatorname{Vomma}^{\mathrm{BS}}\left(x_{0}, \bar{a}_{x_{\mathrm{av}}}^{2} T, k\right) m^{2}\left(\frac{\left[\gamma_{1}\left(a ; x_{\mathrm{av}}\right)_{0}^{T}\right]^{2}+\left[\gamma_{1}\left(\widetilde{a} ; x_{\mathrm{av}}\right)_{0}^{T}\right]^{2}}{2}\right) \\
& +\mathcal{O}\left(\mathcal{M}_{1}(a)\left[\mathcal{M}_{0}(a)\right]^{3} T^{2}\right) .
\end{aligned}
$$

Then write $\quad\left(\frac{\left[\gamma_{1}\left(a ; x_{\mathrm{av}}\right)_{0}^{T}\right]^{2}+\left[\gamma_{1}\left(\tilde{a} ; x_{\mathrm{av}}\right)_{0}^{T}\right]^{2}}{2}\right)=\left(\frac{\gamma_{1}\left(\widetilde{a} ; x_{\mathrm{av}}\right)_{0}^{T}-\gamma_{1}\left(a ; x_{\mathrm{av}}\right)_{0}^{T}}{2}\right)^{2}$ $+\left(\frac{\gamma_{1}\left(\widetilde{a} ; x_{\mathrm{av}}\right)_{0}^{T}+\gamma_{1}\left(a ; x_{\mathrm{av}}\right)_{0}^{T}}{2}\right)^{2}$, use the fact that (see the definition (66) of Vomma ${ }^{\mathrm{BS}}$ and the definition of $\gamma_{1}$ in Definition 21)

$$
\begin{aligned}
& \frac{1}{2} \operatorname{Vomma}^{\mathrm{BS}}\left(x_{0}, \bar{a}_{x_{\mathrm{av}}}^{2} T, k\right) m^{2}\left(\frac{\gamma_{1}\left(\widetilde{a} ; x_{\mathrm{av}}\right)_{0}^{T}+\gamma_{1}\left(a ; x_{\mathrm{av}}\right)_{0}^{T}}{2}\right)^{2} \\
= & \operatorname{Vega~}^{\mathrm{BS}}\left(x_{0}, \bar{a}_{x_{\mathrm{av}}}^{2} T, k\right)\left(C_{1}\left(\widetilde{a} ; x_{\mathrm{av}}\right)_{0}^{T}+C_{1}\left(a ; x_{\mathrm{av}}\right)_{0}^{T}\right)^{2}\left[-\frac{m^{2}}{32 \bar{a}_{x_{\mathrm{av}}}^{5} T^{3}}+\frac{m^{4}}{8 \bar{a}_{x_{\mathrm{av}}}^{9} T^{5}}\right],
\end{aligned}
$$

and finally, use the above identity (obtained with the definitions of $C_{1}, C_{6}$ and with the relation (26)):

$$
\begin{aligned}
&\left(C_{1}(\widetilde{l} ; x)_{0}^{T}+C_{1}(l ; x)_{0}^{T}\right)^{2} \\
&= 2\left[\omega\left(l^{2}(z)\right)_{0}^{T}\right]^{2} C_{6}(l ; z)_{0}^{T}=4 \omega\left(l^{2}(z), l^{2}(z)\right)_{0}^{T} \omega\left(l(z) l^{(1)}(z), l(z) l^{(1)}(z)\right)_{0}^{T} \\
&= 4\left[\omega\left(l(z) l^{(1)}(z), l(z) l^{(1)}(z), l^{2}(z), l^{2}(z)\right)_{0}^{T}+\omega\left(l^{2}(z), l^{2}(z), l(z) l^{(1)}(z), l(z) l^{(1)}(z)\right)_{0}^{T}\right. \\
&+\omega\left(l^{2}(z), l(z) l^{(1)}(z), l(z) l^{(1)}(z), l^{2}(z)\right)_{0}^{T}+\omega\left(l(z) l^{(1)}(z), l^{2}(z), l^{2}(z), l(z) l^{(1)}(z)\right)_{0}^{T} \\
&\left.+\omega\left(l^{2}(z), l(z) l^{(1)}(z), l^{2}(z), l(z) l^{(1)}(z)\right)_{0}^{T}+\omega\left(l(z) l^{(1)}(z), l^{2}(z), l(z) l^{(1)}(z), l^{2}(z)\right)_{0}^{T}\right], \\
& \text { to } \quad \text { cancel } \quad \text { the } \quad \text { terms } \quad \frac{C_{6}\left(a ; x_{\mathrm{av}}\right)_{0}^{T}}{16 \bar{a}_{x_{\mathrm{av}}}^{T}} m^{2}, \quad-\frac{C_{6}\left(a ; x_{\mathrm{av}}\right)_{0}^{T}}{4 \bar{a}_{x_{\mathrm{av}}}^{T} T^{3}} m^{4} \quad \text { and }
\end{aligned}
$$

$\frac{1}{2} \operatorname{Vomma}^{\mathrm{BS}}\left(x_{0}, \bar{a}_{x_{\mathrm{av}}}^{2} T, k\right) m^{2}\left(\frac{\gamma_{1}\left(\widetilde{a} ; x_{\mathrm{av}}\right)_{0}^{T}+\gamma_{1}\left(a ; x_{\mathrm{av}}\right)_{0}^{T}}{2}\right)^{2}$ in (63). That achieves the proof of (59).

In addition to these implied volatility expansions, one can under additional technical assumptions upper bound the residuals terms. For instance, let us consider (57), for which we can prove

$$
\text { Error }_{3, x_{0}}^{\mathrm{I}}=\mathcal{O}\left(\mathcal{M}_{1}(a)\left[\mathcal{M}_{0}(a)\right]^{3} T^{\frac{3}{2}}\right),
$$

which justifies the label of third order expansion. This is available under the assumptions that $|m| \leq \xi \mathcal{M}_{0}(a) \sqrt{T}$ (for a given $\xi \geq 0$ ) and that $\mathcal{M}_{0}(a), \mathcal{M}_{1}(a)$ and $T$ are globally small enough to ensure that the implied volatility approximation 
$\gamma_{0}\left(a ; x_{0}\right)_{0}^{T}-\gamma_{1}\left(a ; x_{0}\right)_{0}^{T} m+\gamma_{2}\left(a ; x_{0}\right)_{0}^{T} m^{2}$ is bounded away from 0 . The method of proof is analogous to that in Subsection 3.4, by performing a third order expansion of BS price w.r.t. the volatility, using the estimate (70) on Ultima ${ }^{\mathrm{BS}}$ (see Corollary $33)$, and carefully gathering terms and evaluating their magnitudes.

\section{Approximation of the Delta}

In this section, we investigate the approximation of the delta of the Call price, i.e. the derivative w.r.t. the spot, by deriving similar expansion formulas. For the sake of brevity we present only results using a log-normal proxy. The results are new. To achieve this goal, we follow again the Dupire approach taking advantage of the symmetry between spot and strike. We start from the Feynman-Kac representation (36) which leads to a nice expression for the delta:

$$
\delta\left(S_{0}, T, K\right)=\partial_{S_{0}} \mathbb{E}\left[\left(S_{0}-e^{k_{T}}\right)_{+}\right]=\mathbb{P}\left(e^{k_{T}}<S_{0}\right)=\mathbb{P}\left(k_{T}<x_{0}\right) .
$$

Thus we are reduced to compute the price of a binary option on the fictitious asset $\left(k_{t}\right)_{t}$. This binary payoff is not anymore differentiable, but we can however apply directly [49, Theorems 2.1, 2.2 and 4.3] to obtain

Theorem 24. (1st and 2nd order approximations for delta using local volatility at strike). Assume $\left(\mathcal{H}^{a}\right)$. Then we have:

$$
\begin{aligned}
\delta\left(e^{x_{0}}, T, e^{k}\right)= & \delta^{\mathrm{BS}}\left(x_{0}, \bar{a}_{k}^{2} T, k\right)+C_{1}(\widetilde{a} ; k)_{0}^{T}\left(\partial_{z^{3}}^{3}-\frac{3}{2} \partial_{z^{2}}^{2}+\frac{1}{2} \partial_{z}\right) \delta^{\mathrm{BS}}\left(x_{0}, \bar{a}_{k}^{2} T, k\right) \\
& +\mathcal{O}\left(\mathcal{M}_{1}(a) \mathcal{M}_{0}(a) T\right), \\
\delta\left(e^{x_{0}}, T, e^{k}\right)= & \delta^{\mathrm{BS}}\left(x_{0}, \bar{a}_{k}^{2} T, k\right)+\sum_{i=1}^{6} \eta_{i}(\widetilde{a} ; k)_{0}^{T} \partial_{k^{i}}^{i} \delta^{\mathrm{BS}}\left(x_{0}, \bar{a}_{k}^{2} T, k\right) \\
& +\mathcal{O}\left(\mathcal{M}_{1}(a)\left[\mathcal{M}_{0}(a)\right]^{2} T^{\frac{3}{2}}\right),
\end{aligned}
$$

where $\delta^{\mathrm{BS}}$ is Black-Scholes delta function defined by $\delta^{\mathrm{BS}}(x, y, z)=\mathcal{N}\left(d_{1}(x, y, z)\right)$, with $x$ the log-spot, $y$ the total variance and $z$ the log-strike.

Remark 25. In view of the error estimate, observe that the corresponding second and third order formulas for vanilla payoffs are respectively first and second order approximations for binary payoffs. This is due to the lack of regularity of the payoff (see our discussion in Subsection 2.5).

Like in the previous price approximation formulas, it is possible to perform additional Taylor expansions in order to obtain similar formulas using local volatility function frozen at spot or at mid-point. We announce two Lemmas which proof is very similar to those of Lemmas 11 and 12 and Theorem 20 is left to the reader. Extra technical results are postponed in Appendix, Subsection 8.3. 
Lemma 26. Let $x \in\left\{x_{0}, x_{\mathrm{av}}\right\}$. Assume $\left(\mathcal{H}^{a}\right)$, then we have

$$
\begin{aligned}
& \delta^{\mathrm{BS}}\left(x_{0}, \bar{a}_{k}^{2} T, k\right) \\
= & \delta^{\mathrm{BS}}\left(x_{0}, \bar{a}_{x}^{2} T, k\right)+2 \partial_{y} \delta^{\mathrm{BS}}\left(x_{0}, \bar{a}_{x}^{2} T, k\right)(k-x) C_{7}(a ; x)_{0}^{T}+\mathcal{O}\left(\mathcal{M}_{1}(a) \mathcal{M}_{0}(a) T\right), \\
= & \delta^{\mathrm{BS}}\left(x_{0}, \bar{a}_{x}^{2} T, k\right)+\left[2(k-x) C_{7}(a ; x)_{0}^{T}+(k-x)^{2} C_{5}(a ; x)_{0}^{T}\right] \partial_{y} \delta^{\mathrm{BS}}\left(x_{0}, \bar{a}_{x}^{2} T, k\right) \\
& +4 \partial_{y^{2}}^{2} \delta^{\mathrm{BS}}\left(x_{0}, \bar{a}_{x}^{2} T, k\right)(k-x)^{2} C_{6}(a ; x)_{0}^{T}+\mathcal{O}\left(\mathcal{M}_{1}(a)\left[\mathcal{M}_{0}(a)\right]^{2} T^{\frac{3}{2}}\right),
\end{aligned}
$$

where $C_{7}(l ; z)_{0}^{T}=\omega\left(l(z) l^{(1)}(z)\right)_{0}^{T}$.

Lemma 27. Let $x \in\left\{x_{0}, x_{\mathrm{av}}\right\}$. Assume $\left(\mathcal{H}^{a}\right)$, then we have

$$
\begin{aligned}
& C_{1}(\widetilde{a} ; k)_{0}^{T}\left(\partial_{z^{3}}^{3}-\frac{3}{2} \partial_{z^{2}}^{2}+\frac{1}{2} \partial_{z}\right) \delta^{\mathrm{BS}}\left(x_{0}, \bar{a}_{k}^{2} T, k\right) \\
&=C_{1}(\widetilde{a} ; x)_{0}^{T}\left(\partial_{z^{3}}^{3}-\frac{3}{2} \partial_{z^{2}}^{2}+\frac{1}{2} \partial_{z}\right) \delta^{\mathrm{BS}}\left(x_{0}, \bar{a}_{x}^{2} T, k\right)+\mathcal{O}\left(\mathcal{M}_{1}(a) \mathcal{M}_{0}(a) T\right), \\
& \sum_{i=1}^{6} \eta_{i}(\widetilde{a} ; k)_{0}^{T} \partial_{z^{i}}^{i}{ }^{\mathrm{BS}}\left(x_{0}, \bar{a}_{k}^{2} T, k\right)=\sum_{i=1}^{6} \eta_{i}(\widetilde{a} ; x)_{0}^{T} \partial_{z^{i}}^{i} \delta^{\mathrm{BS}}\left(x_{0}, \bar{a}_{x}^{2} T, k\right) \\
&+\left[2 C_{6}(\widetilde{a} ; x)_{0}^{T}+C_{2}(\widetilde{a} ; x)_{0}^{T}\right](k-x)\left(\partial_{z^{3}}^{3}-\frac{3}{2} \partial_{z^{2}}^{2}+\frac{1}{2} \partial_{z}\right) \delta^{\mathrm{BS}}\left(x_{0}, \bar{a}_{x}^{2} T, k\right) \\
&+ \\
&+2(k-x) C_{1}(\widetilde{a} ; x)_{0}^{T} C_{7}(\widetilde{a} ; x)_{0}^{T}\left(\partial_{y z^{3}}^{4}-\frac{3}{2} \partial_{y z^{2}}^{3}+\frac{1}{2} \partial_{y z}^{2}\right) \delta^{\mathrm{BS}}\left(x_{0}, \bar{a}_{x}^{2} T, k\right) \\
&+ \mathcal{O}\left(\mathcal{M}_{1}(a)\left[\mathcal{M}_{0}(a)\right]^{2} T^{\frac{3}{2}}\right) .
\end{aligned}
$$

Then remark that:

$$
C_{1}(\widetilde{a} ; x)_{0}^{T} C_{7}(\widetilde{a} ; x)_{0}^{T}=2 C_{4}(\widetilde{a} ; x)_{0}^{T}+C_{8}(\widetilde{a} ; x)_{0}^{T}
$$

where the operator $C_{8}$ is defined as follows:

$$
C_{8}(l ; z)_{0}^{T}=C_{8}(\widetilde{l} ; z)_{0}^{T}=\omega\left(l(z) l^{(1)}(z), l^{2}(z), l(z) l^{(1)}(z)\right)_{0}^{T} .
$$

An application of Proposition 42 finally yields the theorem below.

Theorem 28. (1st and 2nd order approximations for delta using local volatility at spot and mid-point). Assume $\left(\mathcal{H}^{a}\right)$ and let $x \in\left\{x_{0}, x_{\mathrm{av}}\right\}$. We have:

$$
\begin{gathered}
\delta\left(e^{x_{0}}, T, e^{k}\right)=\delta^{\mathrm{BS}}\left(x_{0}, \bar{a}_{x}^{2} T, k\right)+C_{1}(\widetilde{a} ; x)_{0}^{T}\left(\partial_{z^{3}}^{3}-\frac{3}{2} \partial_{z^{2}}^{2}+\frac{1}{2} \partial_{z}\right) \delta^{\mathrm{BS}}\left(x_{0}, \bar{a}_{x}^{2} T, k\right) \\
+(k-x) C_{7}(a ; x)_{0}^{T}\left(\partial_{z^{2}}^{2}-\partial_{z}\right) \delta^{\mathrm{BS}}\left(x_{0}, \bar{a}_{x}^{2} T, k\right)+\mathcal{O}\left(\mathcal{M}_{1}(a) \mathcal{M}_{0}(a) T\right)
\end{gathered}
$$




$$
\begin{aligned}
\delta\left(e^{x_{0}},\right. & \left.T, e^{k}\right)=\delta^{\mathrm{BS}}\left(x_{0}, \bar{a}_{x}^{2} T, k\right)+\sum_{i=1}^{6} \eta_{i}(\widetilde{a} ; k)_{0}^{T} \partial_{z^{i}}^{i} \delta^{\mathrm{BS}}\left(x_{0}, \bar{a}_{x}^{2} T, k\right) \\
& +(k-x)\left[C_{7}(a ; x)_{0}^{T}+\frac{(k-x)}{2} C_{5}(a ; x)_{0}^{T}\right]\left(\partial_{z^{2}}^{2}-\partial_{z}\right) \delta^{\mathrm{BS}}\left(x_{0}, \bar{a}_{x}^{2} T, k\right) \\
& +(k-x)^{2} C_{6}(a ; x)_{0}^{T}\left(\partial_{z^{4}}^{4}-2 \partial_{z^{3}}^{3}+\partial_{z^{2}}^{2}\right) \delta^{\mathrm{BS}}\left(x_{0}, \bar{a}_{x}^{2} T, k\right) \\
& +(k-x)\left[2 C_{6}(\widetilde{a} ; x)_{0}^{T}+C_{2}(\widetilde{a} ; x)_{0}^{T}\right]\left(\partial_{z^{3}}^{3}-\frac{3}{2} \partial_{z^{2}}^{2}+\frac{1}{2} \partial_{z}\right) \delta^{\mathrm{BS}}\left(x_{0}, \bar{a}_{x}^{2} T, k\right) \\
& +(k-x)\left[2 C_{4}(\widetilde{a} ; x)_{0}^{T}+C_{8}(\widetilde{a} ; x)_{0}^{T}\right]\left(\partial_{z^{5}}^{5}-\frac{5}{2} \partial_{z^{4}}^{4}+2 \partial_{z^{3}}^{3}-\frac{1}{2} \partial_{z^{2}}^{2}\right) \delta^{\mathrm{BS}}\left(x_{0}, \bar{a}_{x}^{2} T, k\right) \\
& +\mathcal{O}\left(\mathcal{M}_{1}(a)\left[\mathcal{M}_{0}(a)\right]^{2} T^{\frac{3}{2}}\right) .
\end{aligned}
$$

\section{Numerical experiments}

\subsection{The set of tests}

For the numerical experiments, we consider a CEV model with constant parameters: $\sigma(t, S)=\nu S^{\beta-1}$. We choose a spot value $S_{0}$ equal to 1 and we test two values of $\nu$ (a parameter interpreted as a level of volatility): firstly we set $\nu=0.25$ and we consider either $\beta=0.8$ (a priori close to the log-normal case) or $\beta=0.2$ (a priori close to the normal case). Then we investigate the case of a larger volatility with $\nu=0.4$ and $\beta=0.5$. For the sake of completeness, we give in Appendix 8.5 the expressions of corrective coefficients allowing the computation of our various approximation formulas proposed throughout the paper.

We compare the accuracy of different approximations, for various maturities and various strikes gathered in 5 categories. The strikes evolve approximately as

Table 1. Set of maturities and strikes for the numerical experiments

\begin{tabular}{|l|cc|ccc|cccc|ccc|c|}
\hline $\mathrm{T} / \mathrm{K}$ & \multicolumn{2}{|c|}{ far ITM } & \multicolumn{3}{|c|}{ ITM } & \multicolumn{3}{|c|}{ ATM } & \multicolumn{3}{c|}{ OTM } & far OTM \\
\hline $3 \mathrm{M}$ & 0.70 & 0.75 & 0.80 & 0.85 & 0.90 & 0.95 & 1.00 & 1.05 & 1.10 & 1.15 & 1.25 & 1.30 & 1.35 \\
$6 \mathrm{M}$ & 0.65 & 0.75 & 0.80 & 0.85 & 0.90 & 0.95 & 1.00 & 1.05 & 1.10 & 1.20 & 1.25 & 1.35 & 1.50 \\
$1 \mathrm{Y}$ & 0.55 & 0.65 & 0.75 & 0.80 & 0.90 & 0.95 & 1.00 & 1.05 & 1.15 & 1.25 & 1.40 & 1.50 & 1.80 \\
$1.5 \mathrm{Y}$ & 0.50 & 0.60 & 0.70 & 0.75 & 0.85 & 0.95 & 1.00 & 1.10 & 1.15 & 1.30 & 1.50 & 1.65 & 2.00 \\
$2 \mathrm{Y}$ & 0.45 & 0.55 & 0.65 & 0.75 & 0.85 & 0.90 & 1.00 & 1.10 & 1.20 & 1.35 & 1.55 & 1.80 & 2.30 \\
$3 \mathrm{Y}$ & 0.35 & 0.50 & 0.55 & 0.70 & 0.80 & 0.90 & 1.00 & 1.10 & 1.25 & 1.45 & 1.75 & 2.05 & 2.70 \\
$5 \mathrm{Y}$ & 0.25 & 0.40 & 0.50 & 0.60 & 0.75 & 0.85 & 1.00 & 1.15 & 1.35 & 1.60 & 2.05 & 2.50 & 3.60 \\
$10 \mathrm{Y}$ & 0.15 & 0.25 & 0.35 & 0.50 & 0.65 & 0.80 & 1.00 & 1.20 & 1.50 & 1.95 & 2.75 & 3.65 & 6.30 \\
\hline
\end{tabular}

$S_{0} \exp (c \nu \sqrt{T})$ where $c$ takes the value of various quantiles of the standard Gaussian law (1\%-5\%-10\%-20\%-30\%-40\%-50\%-60\%-70\%-80\%-90\%-95\%-99\%) which allows to cover far ITM and far OTM options. We report in Tables 2, 3 and 4 the BlackScholes implied volatilities corresponding to the exact Call prices with constant parameters [50].

The purpose of the numerical tests is to compare the following approximations: 
Table 2. CEV model $(\beta=0.8, \nu=0.25)$ : BS implied volatilities in $\%$.

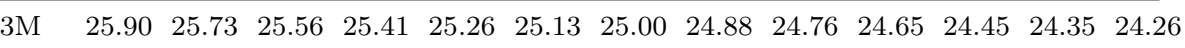

$\begin{array}{llllllllllllll}6 \mathrm{M} & 26.09 & 25.73 & 25.56 & 25.41 & 25.27 & 25.13 & 25.00 & 24.88 & 24.76 & 24.55 & 24.45 & 24.26 & 24.00\end{array}$

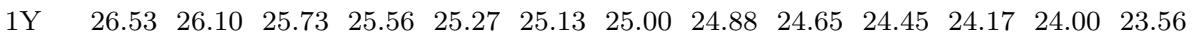

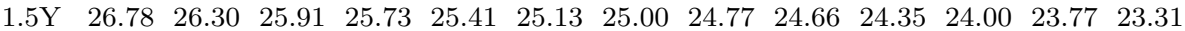

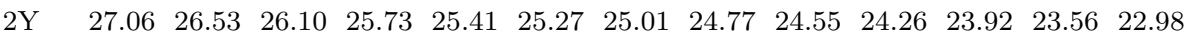

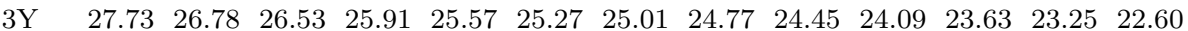

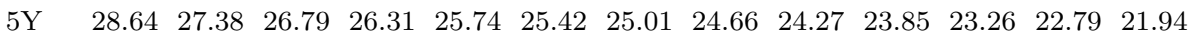

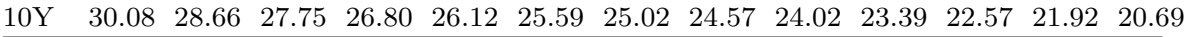

Table 3. CEV model $(\beta=0.2, \nu=0.25)$ : BS implied volatilities in $\%$.

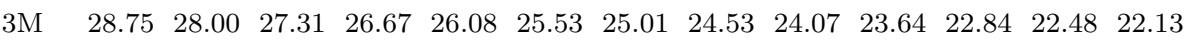

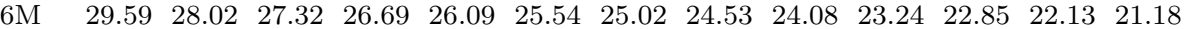

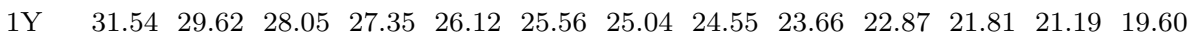

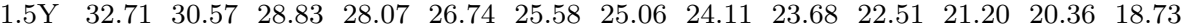

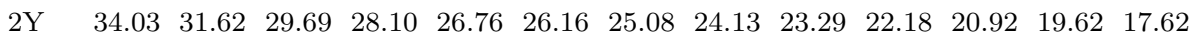

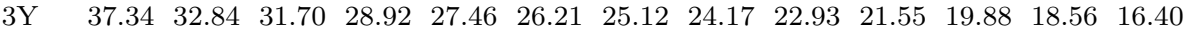

$\begin{array}{llllllllllllll}5 \mathrm{Y} & 42.07 & 35.80 & 33.00 & 30.82 & 28.27 & 26.91 & 25.20 & 23.80 & 22.26 & 20.71 & 18.59 & 17.01 & 14.38\end{array}$

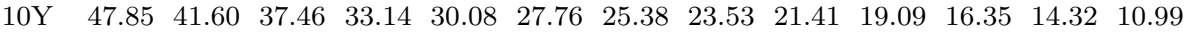

Table 4. CEV model $(\beta=0.5, \nu=0.4)$ : BS implied volatilities in $\%$.

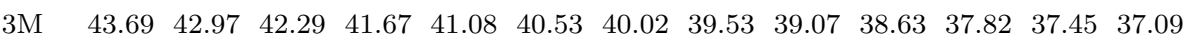
$\begin{array}{llllllllllllll}6 \mathrm{M} & 44.51 & 42.99 & 42.31 & 41.68 & 41.10 & 40.55 & 40.03 & 39.55 & 39.09 & 38.23 & 37.84 & 37.10 & 36.11\end{array}$

$\begin{array}{llllllllllllll}1 \mathrm{Y} & 46.38 & 44.55 & 43.03 & 42.35 & 41.13 & 40.58 & 40.06 & 39.58 & 38.68 & 37.86 & 36.78 & 36.13 & 34.45\end{array}$

$\begin{array}{llllllllllllll}1.5 \mathrm{Y} & 47.49 & 45.46 & 43.80 & 43.06 & 41.75 & 40.61 & 40.10 & 39.14 & 38.71 & 37.51 & 36.15 & 35.27 & 33.52\end{array}$

$\begin{array}{llllllllllllll}2 \mathrm{Y} & 48.73 & 46.47 & 44.63 & 43.10 & 41.79 & 41.20 & 40.13 & 39.17 & 38.31 & 37.17 & 35.87 & 34.49 & 32.31\end{array}$

$\begin{array}{llllllllllllll}3 \mathrm{Y} & 51.76 & 47.62 & 46.55 & 43.90 & 42.48 & 41.26 & 40.18 & 39.22 & 37.97 & 36.54 & 34.79 & 33.36 & 30.97\end{array}$

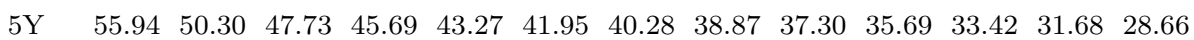

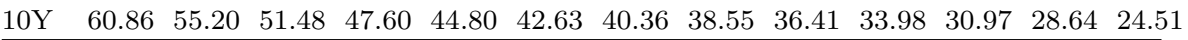

(1) $\operatorname{ImpVol}(\operatorname{AppPriceLN}(2, z))$ and $\operatorname{ImpVol}(\operatorname{AppPriceN}(2, z))$ : the BS implied volatility of the second order expansions based respectively on the log-normal and normal proxy with local volatility frozen at point $z, z$ being respectively equal to $x_{0}, k$ or $x_{\mathrm{av}}$ and to $S_{0}, K$ or $S_{\mathrm{av}}$. See Theorems 9-10-13.

(2) AppImpVolLN $(2, z)$ and $\operatorname{AppImpVolN}(2, z)$ : the second order implied volatility expansions (Theorem 15). All the results are converted into Black-Scholes implied volatility. Namely, for the normal proxy, once we have computed Bachelier implied volatility expansions, we first evaluate the price with the Bachelier formula and then compute the related implied Black-Scholes volatility.

(3) ImpVol (AppPriceLN $(3, z))$ and $\operatorname{ImpVol}(\operatorname{AppPriceN}(3, z))$ : the implied volatility of the third order expansions (Theorems 18 and 19). In addition for the log-normal proxy, we test the average of approximations based on strike and on spot and we denote it by Av. ImpVol (AppPriceLN $(3,)$.$) .$

(4) AppImpVolLN $(3, z)$ and $\operatorname{AppImpVolN}(3, z)$ : the third order implied volatility expansions (Theorem 22). We use the notation Av.AppImpVolLN $(3,$. ) for the 
average of the expansions in strike and in spot.

(5) Hagan and Henry-Labordère formulas denoted by (HF) and (HLF) in the following: benchmark implied volatility approximations of Hagan etal. [5, formula (7) p.149] and Henry-Labordère [6, formula (5.41) p.141]. For the sake of completeness, we recall these well-know implied volatility approximations in the CEV model:

$$
\begin{aligned}
& \sigma_{\mathrm{I}}\left(x_{0}, T, k\right) \approx \nu\left(\frac{S_{0}+K}{2}\right)^{\beta-1}(1+\frac{(1-\beta)(2+\beta)}{6}\left(\frac{S_{0}-K}{S_{0}+K}\right)^{2} \\
&\left.+\frac{(\beta-1)^{2} \nu^{2} T}{24}\left(\frac{S_{0}+K}{2}\right)^{2 \beta-2}\right), \\
& \sigma_{\mathrm{I}}\left(x_{0}, T, k\right) \approx \frac{\nu(1-\beta) \log \left(\frac{S_{0}}{K}\right)}{S_{0}^{1-\beta}-K^{1-\beta}}\left(1+\frac{(\beta-1)^{2} \nu^{2} T}{24}\left(\frac{S_{0}+K}{2}\right)^{2 \beta-2}\right) .
\end{aligned}
$$

We recall that these formulas are essentially available for time-independent volatility, while our formulas allow time dependency.

Our goal is to demonstrate the interest of our approximation formulas in comparison to those of Hagan and Henry-Labordere. We are rather exhaustive with our numerical experiments in order to, on the one hand, select the best approximation formulas among ours, and on the other hand to show that our methods with log-normal proxy involving the mid-point generally outperform Hagan and Henry-Labordère formulas. Full details allow the reader to easily reproduce the results.

In Tables 7 and 9, we report the errors expressed in bps (basis points) on implied volatility for $(\beta, \nu)=(0.8,0.25)$ using the second and the third order price expansions. Tables 8 and 10 give results for the second and the third order implied volatility expansions. Next in Table 11, we report the errors in bps obtained with the averaged expansions Av.ImpVol (AppPriceLN $(3,$.$) )$ and Av.AppImpVolLn $(3,$.$) and the benchmarks (HF) and (HLF). Then in Ta-$ ble 12 , we compare Av. ImpVol (AppPriceLN $(3,)$.$) ) ImpVol \left(\operatorname{AppPriceLN}\left(3, x_{\mathrm{av}}\right)\right)$, Av.AppImpVolLN $(3,$.$) and AppImpVolLN \left(3, x_{\mathrm{av}}\right)$ with the benchmarks $(\mathrm{HF})$ and (HLF).

After we analyse the case $(\beta, \nu)=(0.2,0.25)$ and we report in Tables 13 and 14 the errors using ImpVol (AppPriceLN $\left.\left(3, x_{\mathrm{av}}\right)\right), \operatorname{ImpVol}\left(\operatorname{AppPriceN}\left(3, S_{\mathrm{av}}\right)\right)$, $\operatorname{AppImpVolLN}\left(3, x_{\mathrm{av}}\right), \operatorname{AppImpVolN}\left(3, S_{\mathrm{av}}\right)$ and the benchmarks (HF) and (HLF). Because the other methods in general give globally less accurate results, we just report and compare the best approximations.

Finally in Tables 15 and 16 we establish a comparison between ImpVol (AppPriceLN $\left(3, x_{\mathrm{av}}\right)$ ), AppImpVolLN $\left(3, x_{\mathrm{av}}\right)$ and the benchmarks Hagan and $(\mathrm{HLF})$ for $(\beta, \nu)=(0.5,0.4)$.

For example, on the first row of Table 7 , the value -12 corresponds to the approximation error of $\operatorname{ImpVol}\left(\operatorname{AppPriceLN}\left(2, x_{0}\right)\right)$ for the first strike of the maturity 
$T=3 M$ (i.e. $K=0.7$ ), whereas on the second row, the value -3 corresponds to the approximation error of ImpVol $(\operatorname{AppPriceLN}(2, \mathrm{k}))$ for the third strike of the maturity $T=6 M$ (i.e. $K=0.8$ ). If the price approximation does not belong to the non-arbitrage interval for Call options (it may happen for extremes strikes) we just report ND in the tabular.

\subsection{Analysis of results}

$\triangleright$ Influence of $T$ and $K$. We notice in Tables 7, 8, 9, 10 that errors are increasing w.r.t. $T$ for all the different approximations: this is coherent with the $T^{3 / 2}$ or $T^{2}$ factor of our theoretical error estimates. For ATM options, all the approximations are excellent and errors remain small for a large range of strikes and maturities: with the log-normal proxy, usually smaller than 10 bps up to $10 Y$ for strikes corresponding to the Gaussian quantiles range [10\%, 90\%].

$\triangleright$ Influence of the proxy. As expected, approximations based on log-normal proxy perform better than approximations based on normal proxy. On the one hand, we obtain simpler approximation formulas with the normal proxy: on the other hand, the errors become significant when considering slightly OTM or ITM options, even for short maturities and for advanced methods (order 3, local volatility frozen at the mid-point...).

$\triangleright$ Influence of the order. Regarding firstly Tables 7-9 and then Tables 8-10, we notice that as expected, third order approximations are more accurate than second order ones. In addition, for the log-normal proxy case, second order approximations in spot or strike often underestimate the true implied volatility values whereas third order approximations in spot overestimate the true values for OTM options and yield underestimation for ITM options; the converse occurs for the third order approximations in strike. Because the errors have approximately the same magnitude but with opposite signs, approximations are improved by considering the average between the approximations. It is discussed below.

$\triangleright$ Influence of the point. Unquestionably, methods using the local volatility at mid-point systematically give the best results. With ImpVol (AppPriceLN $\left(2, x_{\mathrm{av}}\right)$ ) (Table 7), errors do not exceed $15 \mathrm{bps}$ for the whole set of strikes and maturities, which is already really good, whereas $\operatorname{ImpVol}\left(\operatorname{AppPriceLN}\left(3, x_{\mathrm{av}}\right)\right.$ ) and AppImpVolLN $\left(3, x_{\text {av }}\right)$ provide errors close to 0 proving an extreme accuracy.

$\triangleright$ Price expansions vs implied volatility expansions. Generally speaking, the implied volatility expansions are more precise and stable. This can be easily observed by comparing on the one hand Tables 7 and 8 and on the other hand Tables 9 and 10. Sometimes, especially for extreme strikes, a simple direct second order second implied volatility expansion is more accurate than the corresponding third order price expansion. Since in addition the formulas are easier to compute, we recommend the use of implied volatility expansions. Moreover, the difference 
between ImpVol (AppPriceLN $\left(3, x_{\mathrm{av}}\right)$ ) and AppImpVolLN $\left(3, x_{\mathrm{av}}\right)$ is not clear, both methods giving similar and excellent results (see Tables 12 or 13) although the direct implied volatility expansion remains more stable especially for $\beta=0.2$ and/or for large maturities. Last, when the local volatility function is frozen at spot or at strike, there is really an improvement in using implied volatility expansions instead of the corresponding price expansions.

$\triangleright$ Comparison with the benchmarks. In Table 11, we report the performance of the methods Av.ImpVol(AppPriceLN $(3,).), \operatorname{Av} \cdot \operatorname{App} \operatorname{ImpVolLN}(3,$.$) and the$ benchmarks (HF) and (HLF). Errors on the implied volatility are equal to zero bp for the whole range of maturities and strikes for $\operatorname{Av} \cdot \operatorname{AppImpVolLN}(3,$.$) and$ the (HLF) approximation, whereas Av.ImpVol (AppPriceLN $(3,$.$) ) and (HF) pro-$ vide errors smaller than 45 and 70 bps in absolute value respectively. In Table 12, we compare Av.ImpVol (AppPriceLN $(3,).), \operatorname{ImpVol}\left(\operatorname{AppPriceLN}\left(3, x_{\mathrm{av}}\right)\right)$, Av.AppImpVolLN $(3,$.$) and AppImpVolLN \left(3, x_{\mathrm{av}}\right)$ with the benchmarks $(\mathrm{HF})$ and (HLF). In order to observe more clearly the accuracy of the different methods, we partially gather the results and we report the average of errors for different categories of strike (far ITM, ITM, ATM, OTM and far OTM, see Table 1), using a scientific notation for the errors. Computing the average per categories of strikes gives an advantage to methods which errors have non constant sign. These methods may be more reliable than those giving a systematic over/under-estimation.

The best method is clearly $\operatorname{AppImpVolLN}\left(3, x_{\mathrm{av}}\right)$ which yields errors of $10^{-5}$ bps for short maturities and $10^{-2}$ bps for long maturities. The method gives better results than the excellent approximation proposed by Henry-Labordère (errors of $10^{-4}$ bps for short maturities and $10^{-1}$ bps for long maturities). ImpVol (AppPriceLN $\left(3, x_{\mathrm{av}}\right)$ ) seems to be slightly better than (HLF) but is less robust for extreme strikes than $\operatorname{App} \operatorname{ImpVolLN}\left(3, x_{\mathrm{av}}\right)$. Significantly better results are obtained by averaging the expansions in spot and strike, thanks to the symmetrical roles played by these two variables. The results are close to those of the corresponding expansions with the mid-point, but they remain less accurate and less robust for extreme strikes. The problem of this averaging method is the risk of huge inaccuracy if one of two approximations in spot and strike fails. (HF) is clearly less accurate than all the other approximations.

$\triangleright$ Influence of $\beta$. In the Table 13, as expected the log-normal proxy provides larger errors than for $\beta=0.8$. Although the results of the normal proxy are better in comparison with the case $\beta=0.8$, they remain less accurate and less robust than those obtained with the log-normal proxy. Up to the maturity $5 Y, \operatorname{AppImpVolLN}\left(3, x_{\mathrm{av}}\right)$ yields errors in bps smaller than 7 bps which is truly excellent. (HLF) gives comparable results. (HF) seems less accurate and cruder for extreme strikes. For the maturity $10 Y$, we observe that AppImpVolLN $\left(3, x_{\text {av }}\right.$ ) (maximal error close to $159 \mathrm{bps}$ ) behaves better than (HLF) (maximal error close to $271 \mathrm{bps}$ ) for very small strikes, whereas for very large strikes (HLF) is slightly better $\left(-5 \mathrm{bps}\right.$ for $\operatorname{AppImpVolLN}\left(3, x_{\mathrm{av}}\right)$ versus -1 bp for (HLF)). Surprisingly (HF) yields the smallest maximal error (close 
to $112 \mathrm{bps}$ ) but is more inaccurate for OTM. (HLF) and AppImpVolLn $\left(3, x_{\mathrm{av}}\right)$ give excellent results with errors of the order of $10^{-3}$ bps for short maturity $(3 M)$ and $10^{-1}$ bps for the maturity $3 Y$. We nevertheless notice that ATM, (HLF) is better.

$\triangleright$ Impact of $\nu$. The level of volatility $\nu$ plays a similar role to $\sqrt{T}$, and in Tables 15 and 16, we analyse the impact of a larger volatility on our approximations. We take $\nu=40 \%$ and $\beta=0.5$. We notice that up to the maturity $5 Y$, the errors in bps do not exceed 6 bps for the methods ImpVol (AppPriceLN $\left(3, x_{\mathrm{av}}\right)$ ) or AppImpVolLN $\left(3, x_{\mathrm{av}}\right)$ with a maximal error of 92 bps for the maturity $10 Y$. Their accuracy is better than those of (HF) or (HLF) for short and long maturities. (HLF) is much more inaccurate ITM for the maturity $10 Y$ (maximal error of $286 \mathrm{bps}$ ). In Table 16, we aggregate the results per categories of strike up to the maturity $3 Y$ and we observe a good accuracy of $\operatorname{ImpVol}\left(\operatorname{AppPriceLN}\left(3, x_{\mathrm{av}}\right)\right)$ and $\operatorname{AppImpVolLN}\left(3, x_{\mathrm{av}}\right): 10^{-3} \mathrm{bps}$ for the maturity $3 M$ and $10^{-1}$ for the maturity $3 Y$. In particular we notice that ATM, (HF) and (HLF) are less accurate.

In view of all these tests, we may conclude that $\operatorname{ImpVol}\left(\operatorname{AppPriceLN}\left(3, x_{\mathrm{av}}\right)\right)$ and particulary AppImpVolLN $\left(3, x_{\text {av }}\right)$ give very satisfying results, being at least as good as the Henry-Labordère formula in the worst situations $(\beta=0.2$ or $\nu=0.4)$ and being often better in the case $\beta=0.8$. The different current tests prove that our direct implied volatility approximations outperform the corresponding price approximations. In addition, a normal proxy seems not to be the most appropriate for the approximation of a CEV model, in view of the large errors obtained especially for very small strikes. This presumably explains why the Hagan formula is much less accurate than our approximations with log-normal proxy and than that of HenryLabordère. The Hagan formula is namely close in the spirit to our approximation formulas with normal proxy.

To conclude, our approximations maintain very tight error estimates and allow to deal naturally with general time-dependent local volatility (or with stochastic interest rates, see [7]) which is a significant advantage compared to other approaches.

\subsection{CEV Delta approximations}

Now we test our approximation formulas for the deltas, by choosing again a CEV model with spot value $S_{0}=1$ and constant parameters. We test the values $(\beta, \nu)=$ $(0.8,0.25)$ and $(\beta, \nu)=(0.2,0.25)$. We report in Tables 5 and 6 the exact delta values for the set of maturities and strikes defined in Table 1.

We test the 6 following approximations:

(1) $\operatorname{AppDeltaLN}\left(1, x_{0}\right), \operatorname{AppDeltaLN}(1, \mathrm{k})$ and $\operatorname{AppDeltaLN}\left(1, x_{\mathrm{av}}\right)$ : first order delta expansions based on the log-normal proxy with local volatility frozen at point $x_{0}, k$ and $x_{\mathrm{av}}$.

(2) $\operatorname{AppDeltaLN}\left(2, x_{0}\right), \operatorname{AppDeltaLN}(2, \mathrm{k})$ and $\operatorname{AppDeltaLN}\left(2, x_{\mathrm{av}}\right)$ : second order delta expansions based on the log-normal proxy with local volatility frozen at 
Table 5. CEV model $(\beta=0.8, \nu=0.25)$ : deltas in $\%$.

$\begin{array}{llllllllllllll}3 \mathrm{M} & 99.75 & 98.89 & 96.38 & 90.83 & 81.18 & 67.67 & 51.99 & 36.60 & 23.57 & 13.91 & 3.78 & 1.76 & 0.76 \\ 6 \mathrm{M} & 99.20 & 95.10 & 90.44 & 83.56 & 74.58 & 64.05 & 52.82 & 41.80 & 31.77 & 16.33 & 11.09 & 4.64 & 1.01 \\ 1 \mathrm{Y} & 99.09 & 96.04 & 88.90 & 83.53 & 69.78 & 61.97 & 53.98 & 46.15 & 31.98 & 20.75 & 9.76 & 5.55 & 0.82 \\ 1.5 \mathrm{Y} & 98.76 & 95.68 & 89.46 & 85.06 & 74.11 & 61.40 & 54.88 & 42.35 & 36.62 & 22.35 & 10.35 & 5.44 & 1.03 \\ 2 \mathrm{Y} & 98.75 & 95.94 & 90.58 & 82.55 & 72.45 & 66.93 & 55.63 & 44.75 & 34.97 & 23.04 & 12.30 & 5.15 & 0.75 \\ 3 \mathrm{Y} & 99.13 & 95.36 & 93.13 & 83.51 & 75.22 & 66.13 & 56.88 & 47.99 & 36.04 & 23.46 & 11.44 & 5.25 & 0.86 \\ 5 \mathrm{Y} & 99.23 & 95.93 & 91.89 & 86.53 & 76.75 & 69.63 & 58.86 & 48.70 & 36.85 & 25.21 & 12.05 & 5.54 & 0.79 \\ 10 \mathrm{Y} & 99.13 & 97.10 & 93.95 & 87.68 & 80.34 & 72.60 & 62.46 & 53.06 & 40.92 & 27.19 & 12.88 & 5.57 & 0.54\end{array}$

Table 6. CEV model $(\beta=0.2, \nu=0.25)$ : deltas in $\%$.

$\begin{array}{llllllllllllll}3 \mathrm{M} & 99.37 & 98.08 & 95.04 & 89.11 & 79.43 & 66.09 & 50.50 & 34.98 & 21.76 & 12.08 & 2.60 & 1.01 & 0.34 \\ 6 \mathrm{M} & 98.13 & 92.97 & 88.01 & 81.08 & 72.24 & 61.88 & 50.71 & 39.59 & 29.35 & 13.70 & 8.60 & 2.85 & 0.35 \\ 1 \mathrm{Y} & 97.34 & 93.15 & 85.45 & 80.09 & 66.66 & 58.97 & 51.00 & 43.08 & 28.51 & 17.00 & 6.39 & 2.91 & 0.14 \\ 1.5 \mathrm{Y} & 96.23 & 92.03 & 85.29 & 80.89 & 70.22 & 57.76 & 51.23 & 38.45 & 32.50 & 17.73 & 6.20 & 2.35 & 0.13 \\ 2 \mathrm{Y} & 95.71 & 91.77 & 85.84 & 77.85 & 68.06 & 62.67 & 51.43 & 40.30 & 30.09 & 17.68 & 7.29 & 1.81 & 0.04 \\ 3 \mathrm{Y} & 95.62 & 90.10 & 87.57 & 77.87 & 69.86 & 61.01 & 51.76 & 42.61 & 30.02 & 16.82 & 5.53 & 1.36 & 0.03 \\ 5 \mathrm{Y} & 94.70 & 89.45 & 84.88 & 79.47 & 70.04 & 63.12 & 52.32 & 41.69 & 28.91 & 16.52 & 4.53 & 0.87 & 0.00 \\ 10 \mathrm{Y} & 94.37 & 90.36 & 86.11 & 79.22 & 71.81 & 64.05 & 53.52 & 43.32 & 29.65 & 14.59 & 2.76 & 0.23 & 0.00\end{array}$

point $x_{0}, k$ and $x_{\mathrm{av}}$.

Tables 17-18 (respectively 19) give errors on deltas (expressed in bps) using all the approximations with $\beta=0.8$ (respectively $\beta=0.2$ ).

Regarding the results, the accuracy for $\beta=0.8$ is excellent because, except for $\operatorname{AppDeltaLN}\left(1, x_{0}\right)$, we obtain a maximal error (in absolute value) equal to $36 \mathrm{bps}$. Generally speaking, approximations with local volatility at spot are not as good as related approximations at strike. In addition, for second order formulas, we do not observe any symmetry between the spot and strike approximations (which often overestimate the exact delta), whereas the symmetry slightly appears for the first order expansions (not exactly with the same magnitude but opposite signs). Maybe in this situation, the optimal expansion point is not exactly the convex combination $x_{\mathrm{av}}=\left(x_{0}+k\right) / 2$. However the methods with the mid-point are truly excellent, in particular $\operatorname{AppDeltaLN}\left(2, x_{\text {av }}\right)$ which yields a maximal error (in absolute value) close to $1 \mathrm{bps}$. From Table 18, we observe that in average, the errors for $\operatorname{AppDeltaLN}\left(2, x_{\mathrm{av}}\right)$ range from $10^{-3}$ for short maturities to $10^{-1}$ for long maturities.

In Table $19(\beta=0.2)$, without surprise the errors are larger compared to $\beta=0.8$. The best approximation is still AppDeltaLN $\left(2, x_{\text {av }}\right)$ which provides errors smaller than 27 bps up to $5 Y$ with a global maximal error of $157 \mathrm{bps}$, which remains quite good. Curiously, for ATM options, the first order approximation may give better estimates even if the related errors quickly for large or small strikes in comparison with the second order approximations. 


\section{Appendix}

\subsection{Computations of derivatives of $\mathrm{Call}^{\mathrm{BS}}$ w.r.t the log spot, the log strike and the total variance}

In the following Proposition, we make explicit the formula for the derivatives at any order of Call ${ }^{\mathrm{BS}}$ w.r.t. $x$ and $z$ :

Proposition 29. Let $x, z \in \mathbb{R}$ and $y>0$. For any integer $n \geq 1$, we have:

$$
\begin{aligned}
\partial_{x^{n}}^{n} \operatorname{Call}^{\mathrm{BS}}(x, y, z) & =e^{x} \mathcal{N}\left(d_{1}(x, y, z)\right) \\
& +\mathbb{1}_{n \geq 2} e^{x} \mathcal{N}^{\prime}\left(d_{1}(x, y, z)\right) \sum_{k=1}^{n-1}\left(\begin{array}{c}
n-1 \\
k
\end{array}\right)(-1)^{k-1} \frac{H_{k-1}\left(d_{1}(x, y, z)\right)}{y^{\frac{k}{2}}}, \\
\partial_{z^{n}}^{z} \operatorname{Call}^{\mathrm{BS}}(x, y, z) & =-e^{z} \mathcal{N}\left(d_{2}(x, y, z)\right) \\
& +\mathbb{1}_{n \geq 2} e^{z} \mathcal{N}^{\prime}\left(d_{2}(x, y, z)\right) \sum_{k=1}^{n-1}\left(\begin{array}{c}
n-1 \\
k
\end{array}\right) \frac{H_{k-1}\left(d_{2}(x, y, z)\right)}{y^{\frac{k}{2}}}
\end{aligned}
$$

where $\left(H_{k}\right)_{k \in \mathbb{N}}$ are the Hermite polynomials defined for any $n \in \mathbb{N}$ and for any $x \in \mathbb{R}$ by:

$$
H_{n}(x)=(-1)^{n} e^{x^{2} / 2} \partial_{x^{n}}^{n}\left(e^{-x^{2} / 2}\right)
$$

Proof. For $n=1$ the formulas are easy to obtain. For $n \geq 2$, apply the Leibniz formula to the products $e^{x} \mathcal{N}\left(d_{1}(x, y, z)\right)$ and $e^{z} \mathcal{N}\left(d_{2}(x, y, z)\right)$.

We deduce a very useful Corollary:

Corollary 30. Let $x, z \in \mathbb{R}$ and $y>0$. For any integers $n \geq 1$ and $m \geq 1$, we have:

$$
\begin{aligned}
& \left|\partial_{x^{n}}^{n} \operatorname{Call}^{\mathrm{BS}}(x, y, z)\right|+\left|\partial_{z^{n}}^{n} \mathrm{Call}^{\mathrm{BS}}(x, y, z)\right| \leq_{c} y^{\frac{1-n}{2}}, \\
& |x-z|^{m}\left|\partial_{x^{n}}^{n} \operatorname{Call}^{\mathrm{BS}}(x, y, z)-e^{x} \mathcal{N}\left(d_{1}(x, y, z)\right)\right| \leq_{c} y^{\frac{1-n+m}{2}}, \\
& |x-z|^{m}\left|\partial_{z^{n}}^{n} \operatorname{Call}^{\mathrm{BS}}(x, y, z)+e^{z} \mathcal{N}\left(d_{2}(x, y, z)\right)\right| \leq_{c} y^{\frac{1-n+m}{2}},
\end{aligned}
$$

where the generic constants depend polynomially on $y$.

Remark 31. In practice the two last estimates are used when we want to bound $(x-z)^{m} \sum_{i=1}^{n} \alpha_{i} \partial_{x^{i}}^{i} \operatorname{Call}^{\mathrm{BS}}(x, y, z)$ or $(x-z)^{m} \sum_{i=1}^{n} \alpha_{i} \partial_{z^{i}}^{i} \mathrm{Call}^{\mathrm{BS}}(x, y, z)\left(\right.$ with $\left.\sum_{i=1}^{n} \alpha_{i}=0\right)$ by a power of $y$ with the highest possible degree.

Proof. We recall that for any polynomial function $\mathcal{P}, x \rightarrow \mathcal{P}(x) \mathcal{N}^{\prime}(x)$ is a bounded function. Then the first inequality follows directly from Proposition 29. For the second and the third, write $(x-z)=d_{1}(x, y, z) \sqrt{y}-\frac{1}{2} y=d_{2}(x, y, z) \sqrt{y}+\frac{1}{2} y$ and conclude similarly. 
In the next Proposition, we provide the formulas of the first, the second and the third derivatives of Call ${ }^{\mathrm{BS}}$ w.r.t. a positive volatility:

Proposition 32. Let $x, z \in \mathbb{R}, \nu>0$ and $T>0$. We have:

$$
\begin{aligned}
& \operatorname{Vega}^{\mathrm{BS}}\left(x, \nu^{2} T, z\right)=\partial_{\nu} \operatorname{Call}^{\mathrm{BS}}\left(x, \nu^{2} T, z\right) \\
& =e^{x} \sqrt{T} \mathcal{N}^{\prime}\left(d_{1}\left(x, \nu^{2} T, z\right)\right)=e^{z} \sqrt{T} \mathcal{N}^{\prime}\left(d_{2}\left(x, \nu^{2} T, z\right)\right), \\
& \operatorname{Vomma}^{\mathrm{BS}}\left(x, \nu^{2} T, z\right)=\partial_{\nu} \operatorname{Vega}^{\mathrm{BS}}\left(x, \nu^{2} T, z\right) \\
& \quad=\frac{\operatorname{Vega}^{\mathrm{BS}}\left(x, \nu^{2} T, z\right)}{\nu} d_{1}\left(x, \nu^{2} T, z\right) d_{2}\left(x, \nu^{2} T, z\right) \\
& \quad=\frac{\operatorname{Vega}^{\mathrm{BS}}\left(x, \nu^{2} T, z\right)}{\nu}\left[\frac{(x-z)^{2}}{\nu^{2} T}-\frac{\nu^{2} T}{4}\right], \\
& \quad=-\frac{\operatorname{Vega}^{\mathrm{BS}}\left(x, \nu^{2} T, z\right)}{\nu^{2}}\left[d_{1} d_{2}\left(1-d_{1} d_{2}\right)+d_{1}^{2}+d_{2}^{2}\right]\left(x, \nu^{2} T, z\right) \\
& \quad=-\frac{\operatorname{Vega}^{\mathrm{BS}}\left(x, \nu^{2} T, z\right)}{\nu^{2}}\left[\frac{(x-z)^{2}}{2}+\frac{3(x-z)^{2}}{\nu^{2} T}+\frac{\nu^{2} T}{4}-\frac{(x-z)^{4}}{\nu^{4} T^{2}}-\frac{\nu^{4} T^{2}}{16}\right] .
\end{aligned}
$$

The above Proposition directly implies the following result:

Corollary 33. Let $x, z \in \mathbb{R}, \nu>0$ and $T>0$. We have the following estimates:

$$
\begin{aligned}
& 0<\operatorname{Vega}^{\mathrm{BS}}\left(x, \nu^{2} T, z\right) \leq_{c} \sqrt{T}, \\
& \left|\operatorname{Vomma}^{\mathrm{BS}}\left(x, \nu^{2} T, z\right)\right| \leq_{c} \frac{\sqrt{T}}{\nu}, \\
& \left|\operatorname{Ultima}^{\mathrm{BS}}\left(x, \nu^{2} T, z\right)\right| \leq_{c} \frac{\sqrt{T}}{\nu^{2}},
\end{aligned}
$$

where the generic constants depend polynomially of $\nu$.

We finally state relations between the derivatives w.r.t. $x$ or $z$, the Vega ${ }^{\mathrm{BS}}$ and the Vomma $^{\mathrm{BS}}$. These relations allow on the one hand to replace derivatives w.r.t. $z$ with derivatives w.r.t. $x$ and on the other hand to write the differential operators w.r.t. $x$ or $z$ in terms of the Vega ${ }^{\mathrm{BS}}$ and the Vomma ${ }^{\mathrm{BS}}$. The verification of these identities is tedious but without mathematical difficulties. For instance, we have used Mathematica to check these relations.

Proposition 34. Let $x, z \in \mathbb{R}, \nu>0$ and $T>0$. We have:

$$
\begin{aligned}
\left(\partial_{x^{2}}^{2}\right. & \left.-\partial_{x}\right) \operatorname{Call}^{\mathrm{BS}}\left(x, \nu^{2} T, z\right)=\left(\partial_{z^{2}}^{2}-\partial_{z}\right) \mathrm{Call}^{\mathrm{BS}}\left(x, \nu^{2} T, z\right) \\
& =\frac{e^{x}}{\nu \sqrt{T}} \mathcal{N}^{\prime}\left(d_{1}\left(x, \nu^{2} T, z\right)\right) \\
& =\frac{\operatorname{Vega}^{\mathrm{BS}}\left(x, \nu^{2} T, z\right)}{\nu T},
\end{aligned}
$$




$$
\begin{aligned}
& \left(\partial_{x^{3}}^{3}-\frac{3}{2} \partial_{x^{2}}^{2}+\frac{1}{2} \partial_{x}\right) \mathrm{Call}^{\mathrm{BS}}\left(x, \nu^{2} T, z\right)=-\left(\partial_{z^{3}}^{3}-\frac{3}{2} \partial_{z^{2}}^{2}+\frac{1}{2} \partial_{z}\right) \mathrm{Call}^{\mathrm{BS}}\left(x, \nu^{2} T, z\right) \\
& =-\frac{e^{x}(x-z)}{\nu^{3} T^{\frac{3}{2}}} \mathcal{N}^{\prime}\left(d_{1}\left(x, \nu^{2} T, z\right)\right) \\
& =-\operatorname{Vega}^{\mathrm{BS}}\left(x, \nu^{2} T, z\right) \frac{(x-z)}{\nu^{3} T^{2}}, \\
& \left(\frac{1}{4} \partial_{x^{4}}^{4}-\frac{1}{2} \partial_{x^{3}}^{3}+\frac{1}{4} \partial_{x^{2}}^{2}\right) \mathrm{Call}^{\mathrm{BS}}\left(x, \nu^{2} T, z\right)=\left(\frac{1}{4} \partial_{z^{4}}^{4}-\frac{1}{2} \partial_{z^{3}}^{3}+\frac{1}{4} \partial_{z^{2}}^{2}\right) \mathrm{Call}^{\mathrm{BS}}\left(x, \nu^{2} T, z\right) \\
& =e^{x} \mathcal{N}^{\prime}\left(d_{1}\left(x, \nu^{2} T, z\right)\right)\left[\frac{(x-z)^{2}}{4 \nu^{5} T^{\frac{5}{2}}}-\frac{1}{16 \nu \sqrt{T}}-\frac{1}{4 \nu^{3} T^{\frac{3}{2}}}\right] \\
& =\operatorname{Vega}^{\mathrm{BS}}\left(x, \nu^{2} T, z\right)\left[\frac{(x-z)^{2}}{4 \nu^{5} T^{3}}-\frac{1}{16 \nu T}-\frac{1}{4 \nu^{3} T^{2}}\right], \\
& \left(\partial_{x^{4}}^{4}-2 \partial_{x^{3}}^{3}+\frac{5}{4} \partial_{x^{2}}^{2}-\frac{1}{4} \partial_{x}\right) \mathrm{Call}^{\mathrm{BS}}\left(x, \nu^{2} T, z\right) \\
& =\left(\partial_{z^{4}}^{4}-2 \partial_{z^{3}}^{3}+\frac{5}{4} \partial_{z^{2}}^{2}-\frac{1}{4} \partial_{z}\right) \mathrm{Call}^{\mathrm{BS}}\left(x, \nu^{2} T, z\right) \\
& =e^{x} \mathcal{N}^{\prime}\left(d_{1}\left(x, \nu^{2} T, z\right)\right)\left[\frac{(x-z)^{2}}{\nu^{5} T^{\frac{5}{2}}}-\frac{1}{\nu^{3} T^{\frac{3}{2}}}\right] \\
& =\operatorname{Vega}^{\mathrm{BS}}\left(x, \nu^{2} T, z\right)\left[\frac{(x-z)^{2}}{\nu^{5} T^{3}}-\frac{1}{\nu^{3} T^{2}}\right], \\
& \left(3 \partial_{x^{4}}^{4}-6 \partial_{x^{3}}^{3}+\frac{7}{2} \partial_{x^{2}}^{2}-\frac{1}{2} \partial_{x}\right) \mathrm{Call}^{\mathrm{BS}}\left(x, \nu^{2} T, z\right) \\
& =\left(3 \partial_{z^{4}}^{4}-6 \partial_{z^{3}}^{3}+\frac{7}{2} \partial_{z^{2}}^{2}-\frac{1}{2} \partial_{z}\right) \mathrm{Call}^{\mathrm{BS}}\left(x, \nu^{2} T, z\right) \\
& =e^{x} \mathcal{N}^{\prime}\left(d_{1}\left(x, \nu^{2} T, z\right)\right)\left[3 \frac{(x-z)^{2}}{\nu^{5} T^{\frac{5}{2}}}-\frac{3}{\nu^{3} T^{\frac{3}{2}}}-\frac{1}{4 \nu \sqrt{T}}\right] \\
& =\operatorname{Vega}^{\mathrm{BS}}\left(x, \nu^{2} T, z\right)\left[3 \frac{(x-z)^{2}}{\nu^{5} T^{3}}-\frac{3}{\nu^{3} T^{2}}-\frac{1}{4 \nu T}\right], \\
& \left(\frac{1}{2} \partial_{x^{6}}^{6}-\frac{3}{2} \partial_{x^{5}}^{5}+\frac{13}{8} \partial_{x^{4}}^{4}-\frac{3}{4} \partial_{x^{3}}^{3}+\frac{1}{8} \partial_{x^{2}}^{2}\right) \mathrm{Call}^{\mathrm{BS}}\left(x, \nu^{2} T, z\right) \\
& =\left(\frac{1}{2} \partial_{z^{6}}^{6}-\frac{3}{2} \partial_{z^{5}}^{5}+\frac{13}{8} \partial_{z^{4}}^{4}-\frac{3}{4} \partial_{z^{3}}^{3}+\frac{1}{8} \partial_{z^{2}}^{2}\right) \mathrm{Call}^{\mathrm{BS}}\left(x, \nu^{2} T, z\right) \\
& =e^{x} \mathcal{N}^{\prime}\left(d_{1}\left(x, \nu^{2} T, z\right)\right)\left[\frac{(x-z)^{4}}{2 \nu^{9} T^{\frac{9}{2}}}-\frac{(x-z)^{2}}{8 \nu^{5} T^{\frac{5}{2}}}-3 \frac{(x-z)^{2}}{\nu^{7} T^{\frac{7}{2}}}+\frac{1}{8 \nu^{3} T^{\frac{3}{2}}}+\frac{3}{2 \nu^{5} T^{\frac{5}{2}}}\right] \\
& =\operatorname{Vega}^{\mathrm{BS}}\left(x, \nu^{2} T, z\right)\left[-3 \frac{(x-z)^{2}}{\nu^{7} T^{4}}+\frac{1}{8 \nu^{3} T^{2}}+\frac{3}{2 \nu^{5} T^{3}}\right] \\
& +\frac{1}{2} \operatorname{Vomma}^{\mathrm{BS}}\left(x, \nu^{2} T, z\right) \frac{(x-z)^{2}}{\nu^{6} T^{4}} \text {. }
\end{aligned}
$$




\subsection{Derivatives of $\mathrm{Call}^{\mathrm{BA}}$ w.r.t the spot, the strike and the total variance}

Proposition 35. Let $S, Z \in \mathbb{R}$ and $Y>0$. For any integer $n \geq 1$, we have:

$$
\begin{aligned}
& \partial_{S^{n}}^{n} \operatorname{Call}^{\mathrm{BA}}(S, Y, Z)=\mathbb{1}_{n=1} \mathcal{N}\left(\frac{S-Z}{\sqrt{Y}}\right)+\mathbb{1}_{n \geq 2} \mathcal{N}^{\prime}\left(\frac{S-Z}{\sqrt{Y}}\right)(-1)^{n-2} \frac{H_{n-2}\left(\frac{S-Z}{\sqrt{Y}}\right)}{Y^{\frac{n-1}{2}}}, \\
& \partial_{Z^{n}}^{n} \operatorname{Call}^{\mathrm{BA}}(S, Y, Z)=-\mathbb{1}_{n=1} \mathcal{N}\left(\frac{S-Z}{\sqrt{Y}}\right)+\mathbb{1}_{n \geq 2} \mathcal{N}^{\prime}\left(\frac{S-Z}{\sqrt{Y}}\right) \frac{H_{n-2}\left(\frac{S-Z}{\sqrt{Y}}\right)}{Y^{\frac{n-1}{2}}} .
\end{aligned}
$$

Corollary 36. Let $S, Z \in \mathbb{R}$ and $Y>0$. For any integers $n \geq 2$ and $m \geq 1$, we have:

$$
|S-Z|^{m}\left(\left|\partial_{S^{n}}^{n} \operatorname{Call}^{\mathrm{BA}}(S, Y, Z)\right|+\left|\partial_{Z^{n}}^{n} \operatorname{Call}^{\mathrm{BA}}(S, Y, Z)\right|\right) \leq_{c} Y^{\frac{1-n+m}{2}},
$$

where the generic constants depend polynomially on $Y$.

Proposition 37. Let $S, Z \in \mathbb{R}, V>0$ and $T>0$. We have:

$$
\begin{aligned}
\operatorname{Vega}^{\mathrm{BA}}\left(S, V^{2} T, Z\right) & =\partial_{V} \operatorname{Call}^{\mathrm{BA}}\left(S, V^{2} T, Z\right)=\sqrt{T} \mathcal{N}^{\prime}\left(\frac{S-Z}{V \sqrt{T}}\right), \\
\operatorname{Vomma}^{\mathrm{BA}}\left(S, V^{2} T, Z\right) & =\partial_{V} \operatorname{Vega}^{\mathrm{BA}}\left(S, V^{2} T, Z\right) \\
& =\frac{\operatorname{Vega}^{\mathrm{BA}}\left(S, V^{2} T, Z\right)}{\nu} \frac{(S-Z)^{2}}{V^{2} T}, \\
\mathrm{Ultima}^{\mathrm{BA}}\left(S, V^{2} T, Z\right) & =\partial_{V} \operatorname{Vomma}^{\mathrm{BA}}\left(S, V^{2} T, Z\right) \\
& =-\frac{\operatorname{Vega}^{\mathrm{BA}}\left(S, V^{2} T, Z\right)}{\nu^{2}}\left[\frac{3(S-Z)^{2}}{V^{2} T}-\frac{(S-Z)^{4}}{V^{4} T^{2}}\right] .
\end{aligned}
$$

Corollary 38. Let $S, Z \in \mathbb{R} V>0$ and $T>0$. We have the following estimates:

$$
\begin{gathered}
0<\operatorname{Vega}^{\mathrm{BA}}\left(S, V^{2} T, Z\right) \leq_{c} \sqrt{T}, \\
\left|\operatorname{Vomma}^{\mathrm{BA}}\left(S, V^{2} T, Z\right)\right| \leq_{c} \frac{\sqrt{T}}{V}, \\
\left|\operatorname{Ultima}^{\mathrm{BA}}\left(S, V^{2} T, Z\right)\right| \leq_{c} \frac{\sqrt{T}}{V^{2}},
\end{gathered}
$$

where the generic constants depend polynomially on $V$. 
Proposition 39. Let $S, Z \in \mathbb{R} V>0$ and $T>0$. We have:

$$
\begin{aligned}
\partial_{S^{2}}^{2} \operatorname{Call}^{\mathrm{BA}}\left(S, V^{2} T, Z\right)= & \partial_{Z^{2}}^{2} \operatorname{Call}^{\mathrm{BA}}\left(x, V^{2} T, Z\right) \\
= & \frac{\operatorname{Vega}^{\mathrm{BA}}\left(S, V^{2} T, Z\right)}{V T}, \\
\partial_{S^{3}}^{3} \operatorname{Call}^{\mathrm{BA}}\left(S, V^{2} T, Z\right)= & -\partial_{Z^{3}}^{3} \operatorname{Call}^{\mathrm{BA}}\left(S, V^{2} T, Z\right) \\
= & -\operatorname{Vega}^{\mathrm{BA}}\left(S, V^{2} T, Z\right) \frac{(S-Z)}{V^{3} T^{2}}, \\
\partial_{S^{4}}^{3} \operatorname{Call}^{\mathrm{BA}}\left(S, V^{2} T, Z\right)= & \partial_{Z^{4}}^{4} \operatorname{Call}^{\mathrm{BA}}\left(S, V^{2} T, Z\right) \\
= & \operatorname{Vega}^{\mathrm{BA}}\left(S, V^{2} T, Z\right)\left[\frac{(S-Z)^{2}}{V^{5} T^{3}}-\frac{1}{V^{3} T^{2}}\right], \\
\partial_{S^{6}}^{6} \mathrm{Call}^{\mathrm{BA}}\left(S, V^{2} T, Z\right)= & \partial_{Z^{6}}^{6} \mathrm{Call}^{\mathrm{BA}}\left(S, V^{2} T, Z\right) \\
= & \operatorname{Vega}^{\mathrm{BA}}(S, V, T, Z)\left[-6 \frac{(S-Z)^{2}}{V^{7} T^{4}}+\frac{3}{V^{5} T^{3}}\right] \\
& +\operatorname{Vomma}^{\mathrm{BA}}\left(S, V^{2} T, Z\right) \frac{(S-Z)^{2}}{V^{6} T^{4}} .
\end{aligned}
$$

\subsection{Derivatives of $\delta^{\mathrm{BS}}$ w.r.t the log spot, the log strike and the total} variance

Proposition 40. Let $x, z \in \mathbb{R}$ and $y>0$. For any integer $n \geq 1$, we have:

$$
\begin{aligned}
& \partial_{x^{n}}^{n} \delta^{\mathrm{BS}}(x, y, z)=(-1)^{n-1} \mathcal{N}^{\prime}\left(d_{1}(x, y, z)\right) \frac{H_{n-1}\left(d_{1}(x, y, z)\right)}{y^{\frac{n}{2}}}, \\
& \partial_{z^{n}}^{n} \delta^{\mathrm{BS}}(x, y, z)=-\mathcal{N}^{\prime}\left(d_{1}(x, y, z)\right) \frac{H_{n-1}\left(d_{1}(x, y, z)\right)}{y^{\frac{n}{2}}} .
\end{aligned}
$$

Corollary 41. Let $x, z \in \mathbb{R}$ and $y>0$. For any integers $n \geq 1$ and $m \geq 1$, we have:

$$
|x-z|^{m}\left(\left|\partial_{x^{n}}^{n} \delta^{\mathrm{BS}}(x, y, z)\right|+\left|\partial_{z^{n}}^{n} \delta^{\mathrm{BS}}(x, y, z)\right|\right) \leq_{c} y^{\frac{m-n}{2}},
$$

where the generic constants depend polynomially on $y$.

Proposition 42. Let $x, z \in \mathbb{R}$ and $y>0$. We have:

$$
\partial_{y} \delta^{\mathrm{BS}}(x, y, z)=\frac{1}{2}\left(\partial_{z^{2}}^{2}-\partial_{z}\right) \delta^{\mathrm{BS}}(x, y, z)=-\frac{\mathcal{N}^{\prime}\left(d_{1}(x, y, z)\right)}{2 y} d_{2}(x, y, z) .
$$

\subsection{Proof of Lemma 4}

We proceed by induction. The key is to prove the above technical result:

Lemma 43. Let $\left(m_{t}\right)_{t \in[0, T]}$ be a square integrable and predictable process, $\left(\lambda_{t}\right)_{t \in[0, T]}$ be a measurable and bounded deterministic function and $\varphi$ be $a C_{b}^{\infty}$ function. Then, 
we have:

$$
\mathbb{E}\left(\varphi\left(\int_{0}^{T} \lambda_{t} \mathrm{~d} W_{t}\right) \int_{0}^{T} m_{t} \mathrm{~d} W_{t}\right)=\mathbb{E}\left(\varphi^{(1)}\left(\int_{0}^{T} \lambda_{t} \mathrm{~d} W_{t}\right) \int_{0}^{T} \lambda_{t} m_{t} \mathrm{~d} t\right) .
$$

Proof. We propose two proofs: firstly we employ a PDE argument and secondly we show that this is a straightforward application of the Malliavin calculus theory. In the two points of view, we use the common notation for the diffusion process $\left(Z_{t}\right)_{t \in[0, T]}=\left(\int_{0}^{t} \lambda_{s} \mathrm{~d} W_{s}\right)_{t \in[0, T]}$ and we recall that $\left(\mathcal{F}_{t}\right)_{t \in[0, T]}$ denotes the augmented filtration of the Brownian motion $W$.

$\triangleright$ PDE argument. We introduce $u(t, x)=\mathbb{E}\left[\varphi\left(Z_{T}\right) \mid Z_{t}=x\right]$ which solves the following PDE with terminal condition:

$$
\left\{\begin{array}{l}
\left.\partial_{t} u(t, x)+\frac{1}{2} \lambda_{t}^{2} \partial_{x x}^{2} u(t, x)=0, \quad(t, x) \in\right] 0, T[\times \mathbb{R}, \\
u(T, x)=\varphi(x), \quad x \in \mathbb{R} .
\end{array}\right.
$$

Thanks to the above PDE and the assumption on $\varphi, \forall i \in \mathbb{N}, \partial_{x^{i}}^{i}\left(u\left(t, Z_{t}\right)\right)_{t \in[0, T]}$ is a martingale and $\forall t \in[0, T]$, we have:

$$
\partial_{x^{i}}^{i} u\left(t, Z_{t}\right)=\mathbb{E}\left[\varphi^{(i)}\left(Z_{T}\right) \mid \mathcal{F}_{t}\right]=\mathbb{E}\left[\varphi^{(i)}\left(Z_{T}\right)\right]+\int_{0}^{t} \partial_{x^{i+1}}^{i+1} u\left(s, Z_{s}\right) \lambda_{s} \mathrm{~d} W_{s} .
$$

Then applying the $L_{2}$-isometry for the product $u\left(T, Z_{T}\right) \int_{0}^{T} m_{t} \mathrm{~d} W_{t}=$ $\varphi\left(Z_{T}\right) \int_{0}^{T} m_{t} \mathrm{~d} W_{t}$, it readily comes:

$$
\mathbb{E}\left(\varphi\left(Z_{T}\right) \int_{0}^{T} m_{t} \mathrm{~d} W_{t}\right)=\int_{0}^{T} \mathbb{E}\left[\partial_{x} u\left(t, Z_{t}\right) \lambda_{t} m_{t}\right] \mathrm{d} t=\mathbb{E}\left(\varphi^{(1)}\left(Z_{T}\right) \int_{0}^{T} \lambda_{t} m_{t} \mathrm{~d} t\right),
$$

where at the last equality we have used the martingale property of $\partial_{x}\left(u\left(t, Z_{t}\right)\right)_{t \in[0, T]}$. $\triangleright$ Malliavin calculus approach. The result directly comes from the duality relationship of Malliavin calculus (see [48, Lemma 1.2.1]) identifying the Itô integral $\int_{0}^{T} m_{t} \mathrm{~d} W_{t}$ with the Skorohod operator and observing that $\left(\varphi^{(1)}\left(Z_{T}\right) \lambda_{t}\right)_{t \in[0, T]}$ is the first Malliavin derivative of $\varphi\left(Z_{T}\right)$.

Lemma 43 is a particular case of Lemma 4 for $N=1$ and $I_{N}=1$ noting that $\forall i \in \mathbb{N}$, $\mathbb{E}\left(\varphi^{(i)}\left(\int_{0}^{T} \lambda_{t} \mathrm{~d} W_{t}\right)\right)=\left.\partial_{\varepsilon^{i}}^{i} \mathbb{E}\left(\varphi\left(\int_{0}^{T} \lambda_{t} \mathrm{~d} W_{t}+\varepsilon\right)\right)\right|_{\varepsilon=0}$, thanks to the regularity of $\varphi$. For $N=1$ and $I_{N}=0$, there is nothing to prove. Suppose that the formula (34) is true for $N \geq 2$. Then apply Lemma 43 if $I_{N+1}=1$ to obtain:

$$
\begin{aligned}
& \mathbb{E}\left(\varphi\left(\int_{0}^{T} \lambda_{t} \mathrm{~d} W_{t}\right) \int_{0}^{T} l_{N+1, t_{N+1}} \int_{0}^{t_{N+1}} l_{N, t_{N}} \ldots \int_{0}^{t_{2}} l_{1, t_{1}} \mathrm{~d} W_{t_{1}}^{I_{1}} \ldots \mathrm{d} W_{t_{N}}^{I_{N}} \mathrm{~d} W_{t_{N+1}}^{I_{N+1}}\right) \\
= & \mathbb{E}\left(\varphi^{\left(I_{N+1}\right)}\left(\int_{0}^{T} \lambda_{t} \mathrm{~d} W_{t}\right) \int_{0}^{T} \widehat{l}_{N+1, t_{N+1}}\right. \\
& \left.\times \int_{0}^{t_{N+1}} l_{N, t_{N}} \int_{0}^{t_{N}} \ldots \int_{0}^{t_{2}} l_{1, t_{1}} \mathrm{~d} W_{t_{1}}^{I_{1}} \ldots \mathrm{d} W_{t_{N}}^{I_{N}} \mathrm{~d} t_{N+1}\right) \\
= & \mathbb{E}\left(\varphi^{\left(I_{N+1}\right)}\left(\int_{0}^{T} \lambda_{t} \mathrm{~d} W_{t}\right) \int_{0}^{T}\left(l_{N, t_{N}} \int_{t_{N}}^{T} \widehat{l}_{N+1, s} \mathrm{~d} s\right) \int_{0}^{t_{N}} \ldots \int_{0}^{t_{2}} l_{1, t_{1}} \mathrm{~d} W_{t_{1}}^{I_{1}} \ldots \mathrm{d} W_{t_{N}}^{I_{N}}\right),
\end{aligned}
$$


where at the last equality we have used the fact that $\int_{0}^{T} f_{t} Z_{t} \mathrm{~d} t=\int_{0}^{T}\left(\int_{t}^{T} f_{s} \mathrm{~d} s\right) d Z_{t}$ for any continuous semi-martingale $Z$ starting from 0 and any measurable and bounded deterministic function $f$ (apply the Itô formula to the product $\left.\left(\int_{t}^{T} f_{s} d s\right) Z_{t}\right)$. We easily conclude with the induction hypothesis and leave the details to the reader.

\subsection{Applications of the expansions for time-independent CEV model}

We specify in this section the results and the practical calculus of the various expansion coefficients when the volatility has the form:

$$
\sigma(S)=\nu S^{\beta-1},
$$

i.e. a CEV-type time-independent volatility with a level $\nu$ and a skew $\beta \leq 1$. Although the volatility and its derivatives are not bounded, we expect that our expansions can be generalized to that model. Alternatively, to fit our assumptions, we would need to modify the CEV volatility function $\sigma$ near 0 and $+\infty$, so that the ellipticity and regularity conditions are met. The impact of such a modification has been studied in the case of Limited CEV model in [51] where the authors show a very small impact on prices. Observe in addition that the correction terms in our expansions do no depend on the modification of $\sigma$ at 0 and $+\infty$.

To apply our different expansion theorems, we need to give the expressions of the coefficients $\left(C_{i}\right)_{1 \leq i \leq 8}$ defined in Definition 17, in Theorem 20 and in Lemmas 26-27. A straightforward calculus leads to:

$$
\begin{aligned}
& a(x)=\nu e^{x(\beta-1)}, \quad a^{(1)}(x)=(\beta-1) a(x), \quad a^{(2)}(x)=(\beta-1)^{2} a(x), \\
& a\left(x_{0}\right)=\nu S_{0}^{\beta-1}, \quad a(k)=\nu K^{\beta-1}, \quad a\left(x_{\mathrm{av}}\right)=\nu\left(S_{0} K\right)^{\frac{\beta-1}{2}}, \\
& \Sigma(S)=\nu S^{\beta}, \quad \Sigma^{(1)}(S)=\beta \frac{\Sigma(S)}{S}, \quad \Sigma^{(2)}(S)=\beta(\beta-1) \frac{\Sigma(S)}{S^{2}}, \\
& \Sigma\left(S_{0}\right)=\nu S_{0}^{\beta}, \quad \Sigma(K)=\nu K^{\beta}, \quad \Sigma\left(S_{\mathrm{av}}\right)=\nu\left(\frac{S_{0}+K}{2}\right)^{\beta} .
\end{aligned}
$$

Thus for $\beta \in[0,1]$, the magnitudes of $\mathcal{M}_{0}(a)$ and $\mathcal{M}_{1}(a)$ are mainly linked to those of $\nu$ and $\nu(\beta-1)$. At the limit case $\beta=1$, the model coincides with the log-normal proxy and $\mathcal{M}_{1}(a)=0$. In the same spirit, $\nu$ and $\nu \beta$ are respectively linked to $\mathcal{M}_{0}(\Sigma)$ and $\mathcal{M}_{1}(\Sigma)$. At the limit case $\beta=0$, the model coincides with the normal proxy and $\mathcal{M}_{1}(\Sigma)=0$.

Finally, the expression of the coefficients $\left(C_{i}\right)_{1 \leq i \leq 8}$ are:

$$
\begin{array}{ll}
C_{1}(a ; x)_{0}^{T}=(\beta-1) a^{4}(x) \frac{T^{2}}{2}, & C_{2}(a ; x)_{0}^{T}=(\beta-1)^{2} a^{4}(x) T^{2}, \\
C_{3}(a ; x)_{0}^{T}=(\beta-1)^{2} a^{6}(x) \frac{T^{3}}{3}, & C_{4}(a ; x)_{0}^{T}=C_{8}(a ; x)_{0}^{T}=(\beta-1)^{2} a^{6}(x) \frac{T^{3}}{6} \\
C_{5}(a ; x)_{0}^{T}=2(\beta-1)^{2} a^{2}(x) T, & C_{6}(a ; x)_{0}^{T}=(\beta-1)^{2} a^{4}(x) \frac{T^{2}}{2},
\end{array}
$$




$$
\begin{array}{rlrl}
C_{7}(a ; x)_{0}^{T} & =(\beta-1) a^{2}(x) T, & \\
C_{1}(\Sigma ; S)_{0}^{T} & =\beta \frac{\Sigma^{4}(S)}{S} \frac{T^{2}}{2}, & C_{2}(\Sigma ; S)_{0}^{T}=\beta(2 \beta-1) \frac{\Sigma^{4}(S)}{S^{2}} \frac{T^{2}}{2}, \\
C_{3}(\Sigma ; S)_{0}^{T}=\beta(2 \beta-1) \frac{\Sigma^{6}(S)}{S^{2}} \frac{T^{3}}{6}, & C_{4}(\Sigma ; S)_{0}^{T}=C_{8}(\Sigma ; S)_{0}^{T}=\beta^{2} \frac{\Sigma^{6}(S)}{S^{2}} \frac{T^{3}}{6}, \\
C_{5}(\Sigma ; S)_{0}^{T}=\beta(2 \beta-1) \frac{\Sigma^{2}(S)}{S^{2}} T, & C_{6}(\Sigma ; S)_{0}^{T}=\beta^{2} \frac{\Sigma^{4}(S)}{S^{2}} \frac{T^{2}}{2}, \\
C_{7}(\Sigma ; S)_{0}^{T}=\beta \frac{\Sigma^{2}(S)}{S} T, &
\end{array}
$$

where $x=x_{0}, k, x_{\mathrm{av}}$ and $S=S_{0}, K, S_{\mathrm{av}}$.

We now give the expressions of the coefficients $\gamma_{i}, \pi_{i}, \chi_{i}$ and $\Xi_{i}$ defined in Definition 21 useful to compute the implied volatility expansions:

$$
\begin{aligned}
\gamma_{0}(a ; x)_{0}^{T}=\frac{(\beta-1)^{2}}{24} a^{3}(x) T\left[1-\frac{a^{2}(x) T}{4}\right], & \gamma_{1}(a ; x)_{0}^{T}=\frac{(\beta-1)}{2} a(x), \\
\gamma_{2}(a ; x)_{0}^{T}=\frac{(\beta-1)^{2}}{12} a(x), & \pi_{0}(a ; x)_{0}^{T}=\gamma_{0}(a ; x)_{0}^{T}, \\
\pi_{1}(a ; x)_{0}^{T}=0, & \pi_{2}(a ; x)_{0}^{T}=-\frac{(\beta-1)^{2}}{24} a(x), \\
\chi_{1}(\Sigma ; S)_{0}^{T}=\frac{\beta \Sigma(S)}{2 S}, & \chi_{0}(\Sigma ; S)_{0}^{T}=\frac{\beta(\beta-2)}{24 S^{2}} \Sigma^{3}(S) T, \\
\chi_{2}(\Sigma ; S)_{0}^{T}=\frac{\beta(\beta-2)}{12 S^{2}} \Sigma(S), & \Xi_{0}(\Sigma ; S)_{0}^{T}=\chi_{0}(\Sigma ; S)_{0}^{T}, \\
\Xi_{2}(\Sigma ; S)_{0}^{T}=-\frac{\beta(\beta+1)}{24 S^{2}} \Sigma(S), & \Xi_{1}(\Sigma ; S)_{0}^{T}=0 .
\end{aligned}
$$

For example, the second and third order Black-Scholes and Bachelier implied volatility expansions based on the mid-points are explicitely given by:

$$
\begin{aligned}
\sigma_{\mathrm{I}}\left(x_{0}, T, k\right) \approx \nu\left(S_{0} K\right)^{\frac{\beta-1}{2}}, & \\
\sigma_{\mathrm{I}}\left(x_{0}, T, k\right) \approx \nu\left(S_{0} K\right)^{\frac{\beta-1}{2}}[1 & +\frac{(\beta-1)^{2} \nu^{2} T}{24}\left(S_{0} K\right)^{\beta-1}\left(1-\frac{\nu^{2} T\left(S_{0} K\right)^{\beta-1}}{4}\right) \\
& \left.-\frac{(\beta-1)^{2}}{24} \log ^{2}\left(\frac{S_{0}}{K}\right)\right], \\
\Sigma_{\mathrm{I}}\left(S_{0}, T, K\right) \approx \nu\left(\frac{S_{0}+K}{2}\right)^{\beta}, & \\
\Sigma_{\mathrm{I}}\left(S_{0}, T, K\right) \approx \nu\left(\frac{S_{0}+K}{2}\right)^{\beta}\left[1+\frac{\beta(\beta-2) \nu^{2} T}{24}\left(\frac{S_{0}+K}{2}\right)^{2 \beta-2}\right. & \left.-\frac{\beta(\beta+1)}{6}\left(\frac{S_{0}-K}{S_{0}+K}\right)^{2}\right]
\end{aligned}
$$

which are very simple formulas. The last formula coincides with the intermediate equation (A.28b) in [5]. 


\section{References}

[1] Y. Achdou and O. Pironneau, Computational Methods for Option Pricing. (SIAM series, Frontiers in Applied Mathematics, Philadelphia, 2005).

[2] P. Glasserman, Monte Carlo Methods in Financial Engineering. (Springer, 2003).

[3] P. Carr and D. Madan, Option valuation using the fast Fourier transform, Journal of Computational Finance. 2(4), 61-73, (1998).

[4] A. Lewis, Option valuation under stochastic volatility. (Finance Press, 2000).

[5] P. Hagan and D. Woodward, Equivalent Black volatilities, Applied Mathematical Finance. 6, 147-157, (1999).

[6] P. Henry-Labordère, Analysis, Geometry, and Modeling in Finance: Advanced Methods in Option Pricing. (Chapman and Hall, 2008).

[7] E. Benhamou, E. Gobet, and M. Miri, Analytical formulas for local volatility model with stochastic rates, Quantitative Finance. 12(2), 185-198, (2012).

[8] W. Schachermayer and J. Teichmann, How close are the option pricing formulas of Bachelier and Black-Merton-Scholes?, Math. Finance. 18(1), 155-170, (2008).

[9] M. Musiela and M. Rutkowski, Martingale methods in financial modelling. (Springer Verlag, 2005), second edition.

[10] R. Lee. Implied volatility: Statics, dynamics, and probabilistic interpretation. In Recent Advances in Applied Probability. Springer, (2005).

[11] S. Benaim and P. Friz, Regular variation and smile asymptotics, Mathematical Finance. 19(1), 1-12, (2009).

[12] A. Gulisashvili, Asymptotic formulas with error estimates for call pricing functions and the implied volatility at extreme strikes, SIAM J. Financial Math. 1, 609-641, (2010).

[13] A. Gulisashvili, Ed., Analytically Tractable Stochastic Stock Price Models. (Forthcoming in Springer Finance, 2012).

[14] M. Tehranchi, Asymptotics of implied volatility far from maturity, Journal of Applied Probability. 46(3), 629-650, (2009).

[15] L. Rogers and M. Tehranchi, Can the implied volatility surface move by parallel shifts?, Finance and Stochastics. 14, 235-248, (2010).

[16] M. Forde and A. Jacquier, The large-maturity smile for the Heston model, Finance and Stochastics. 15(4), 755-780, (2011).

[17] A. Jacquier, M. Keller-Ressel, and A. Mijatovic, Large deviations in stochastic volatility with jumps: asymptotic implied volatility for affine models, preprint, http: //arxiv. org/abs/1108. 3998. (2011).

[18] J. Gatheral and A. Jacquier, Convergence of Heston to SVI, Quantitative Finance. 11(8), 1129-1132, (2011).

[19] J. Gatheral, A parsimonious arbitrage-free implied volatility parameterization with application to the valuation of volatility derivatives, Presentation at Global Derivatives $\mathcal{G}$ Risk Management, Madrid, www. math. nyu. edu/fellows_fin_math/ gatheral/madrid2004. pdf (May. 2004).

[20] D. Duffie, D. Filipovic, and W. Schachermayer, Affine processes and applications in finance, The Annals of Applied Probability. 13(3), 984-1053, (2003).

[21] M. Freidlin and A. Wentzell, Random perturbations of dynamical systems. (New York, NY: Springer, 1998), second edition. Transl. from the Russian by Joseph Szuecs.

[22] W. Fleming and P. Souganidis, Asymptotic series and the method of vanishing viscosity, Indiana Univ. Math. J. 35, 425-447, (1986).

[23] E. Fournié, J. Lebuchoux, and N. Touzi, Small noise expansion and importance sampling, Asymptot. Anal. 14(4), 361-376, (1997). 
[24] J. Fouque, G. Papanicolaou, R. Sircar, and S. Knut, Multiscale stochastic volatility for equity, interest rate, and credit derivatives. (Cambridge University Press, Cambridge, 2011).

[25] R. Azencott, Densité des diffusions en temps petit: développements asymptotiques. partie i, Séminaire de Probabilités XVIII - Lecture Notes in Mathematics 1059. (1982$83)$.

[26] S. Watanabe, Analysis of Wiener functionals (Malliavin calculus) and its applications to heat kernels, Annals of Probability. 15(1), 1-39, (1987).

[27] N. Yoshida, Asymptotic expansions of maximum likelihood estimator for small diffusion via the theory of Malliavin-Watanabe, Probability Theory and Related Fields. 92, 275-311, (1992).

[28] N. Yoshida, Asymptotic expansion for statistics related to small diffusions, J. Japan Statistical Society. 22(2), 139-159, (1992).

[29] M. Uchida and N. Yoshida, Asymptotic expansion for small diffusions applied to option pricing, Statistical Inference for Stochastics Processes. 7, 189-223, (2004).

[30] N. Kunitomo and A. Takahashi, The asymptotic expansion approach to the valuation of interest rate contingent claims, Mathematical Finance. 11(1), 117-151, (2001).

[31] Y. Osajima, The asymptotic expansion formula of implied volatility for dynamic SABR model and FX hybrid model, Available at http://ssrn. com/abstract= 965265. (2007).

[32] E. Alòs and C. Ewald, Malliavin differentiability of the Heston volatility and applications to option pricing, Advances in Applied Probability. 40(1), 144-162, (2008).

[33] P. Hagan, D. Kumar, A. Lesniewski, and D. Woodward, Managing smile risk, Willmott Magazine. pp. 84-108, (2002).

[34] P. Kloeden and E. Platen, Numerical solution of stochastic differential equations. (Springer Verlag, 1995).

[35] G. Papanicolaou and R. Sircar, Stochastic volatility, smile and asymptotics, Applied Mathematical Finance. 6(2), 107-145, (1999).

[36] J. Fouque, G. Papanicolaou, and R. Sircar, Derivatives in financial Markets with stochastic volatility. (Cambridge University Press, 2000).

[37] L. Scott, Option pricing when the variance changes randomly : Theory, estimation, and an application, J. Financial and Quantitative Analysis. 22, 419-438, (1987).

[38] J. Fouque, G. Papanicolaou, R. Sircar, and S. Solna, Singular perturbations in option pricing, SIAM J. Appl. Math. 63(5), 1648-1665, (2003).

[39] J. Fouque, G. Papanicolaou, R. Sircar, and K. Solna, Maturity cycles in implied volatility, Finance and Stochastics. 8(4), 451-477, (2004).

[40] J. Kevorkian and J. Cole, Perturbation Methods in Applied Mathematics. (SpringerVerlag, 1985).

[41] H. Kunita, Stochastic flows of diffeomorphisms, École d'été de Probabilits de St-Flour XII. Lecture notes. 1097, (1984).

[42] E. Gobet and M. Miri, Weak approximations of averaged diffusion processes, Forthcoming in Stochastic Processes and their Applications. (2012).

[43] B. Dupire, Pricing with a smile, Risk. 7(1), 18-20, (1994).

[44] E. Gobet and A. Suleiman. New approximations in local volatility models. In ed. Y. Kabanov, Musiela Festschrift. Springer, (2012).

[45] E. Benhamou, E. Gobet, and M. Miri, Smart expansion and fast calibration for jump diffusion, Finance and Stochastics. 13(4), 563-589, (2009).

[46] E. Benhamou, E. Gobet, and M. Miri, Time dependent Heston model, SIAM Journal on Financial Mathematics. 1, 289-325, (2010).

[47] R. Bompis and E. Gobet, Price expansion formulas for model combining local and 
stochastic volatility, Preprint. (2012).

[48] D. Nualart, Malliavin calculus and related topics. (Springer Verlag, 2006), second edition.

[49] E. Benhamou, E. Gobet, and M. Miri, Expansion formulas for European options in a local volatility model, International Journal of Theoretical and Applied Finance. 13 (4), 603-634, (2010).

[50] M. Schroder, Computing the constant elasticity of variance option pricing formula, Journal of Finance. 44, 211-219, (1989).

[51] L. Andersen and J. Andreasen, Volatility skews and extensions of the Libor market model, Applied Mathematical Finance. 7(1), 1-32, (2000). 
Table 7. CEV model $(\beta=0.8, \nu=0.25)$ : errors in bps on the BS implied volatility using the 6 second order price approximations ImpVol (AppPriceLN $\left.\left(2, x_{0}\right)\right), \operatorname{ImpVol}(\operatorname{AppPriceLN}(2, \mathrm{k})), \operatorname{ImpVol}\left(\operatorname{AppPriceLN}\left(2, x_{\mathrm{av}}\right)\right)$, ImpVol (AppPriceN $\left(2, S_{0}\right)$ ), ImpVol (AppPriceN $\left.(2, K)\right)$ and ImpVol (AppPriceN $\left(2, S_{\text {av }}\right)$ ).

\begin{tabular}{|c|c|c|c|c|c|c|c|c|c|c|c|c|c|}
\hline \multirow{6}{*}{$3 \mathrm{M}$} & -12 & -6 & -2 & -1 & 0 & 0 & 0 & 0 & 0 & -1 & -3 & -5 & -8 \\
\hline & -17 & -7 & -3 & -1 & 0 & 0 & 0 & 0 & 0 & -1 & -2 & -4 & -7 \\
\hline & 0 & 0 & 0 & 0 & 0 & 0 & 0 & 0 & 0 & 0 & 0 & 0 & 0 \\
\hline & -577 & -79 & -18 & -1 & 2 & 2 & 2 & 2 & 2 & 0 & -22 & -44 & -73 \\
\hline & -124 & -59 & -20 & -2 & 2 & 2 & 2 & 2 & 2 & 1 & -21 & -53 & -125 \\
\hline & 21 & 14 & 9 & 6 & 3 & 2 & 2 & 2 & 3 & 4 & 9 & 11 & 15 \\
\hline \multirow[t]{6}{*}{$\overline{6 \mathrm{M}}$} & -13 & -3 & -1 & -1 & 0 & 0 & 0 & 0 & 0 & -1 & -2 & -4 & -15 \\
\hline & -17 & -4 & -2 & -1 & 0 & 0 & 0 & 0 & 0 & -1 & -2 & -4 & -11 \\
\hline & 1 & 0 & 0 & 0 & 0 & 0 & 0 & 0 & 0 & 0 & 0 & 0 & 1 \\
\hline & -269 & -18 & 0 & 5 & 5 & 4 & 3 & 4 & 4 & 1 & -5 & -35 & -117 \\
\hline & -138 & -26 & -4 & 4 & 5 & 4 & 3 & 4 & 4 & 3 & -2 & -30 & -238 \\
\hline & 33 & 16 & 11 & 7 & 5 & 4 & 3 & 3 & 4 & 8 & 10 & 16 & 26 \\
\hline \multirow[t]{6}{*}{$\overline{1 Y}$} & -23 & -8 & -2 & -1 & 0 & 0 & 0 & 0 & 0 & -1 & -4 & -8 & -37 \\
\hline & -34 & -9 & -2 & -1 & 0 & 0 & 0 & 0 & 0 & -1 & -4 & -7 & -23 \\
\hline & 1 & 1 & 0 & 0 & 0 & 0 & 0 & 0 & 0 & 0 & 0 & 0 & 1 \\
\hline & -848 & -48 & 6 & 10 & 8 & 7 & 6 & 7 & 9 & 6 & -21 & -59 & -235 \\
\hline & -240 & -69 & -1 & 8 & 8 & 7 & 6 & 7 & 9 & 9 & -9 & -45 & ND \\
\hline & 64 & 36 & 20 & 14 & 8 & 7 & 6 & 7 & 9 & 13 & 22 & 29 & 54 \\
\hline \multirow[t]{6}{*}{$1.5 \mathrm{Y}$} & -28 & -11 & -3 & -2 & -1 & 0 & 0 & 0 & -1 & -2 & -6 & -12 & -50 \\
\hline & -41 & -12 & -4 & -2 & -1 & 0 & 0 & 0 & -1 & -2 & -5 & -10 & -30 \\
\hline & 2 & 1 & 0 & 0 & 0 & 0 & 0 & 0 & 0 & 0 & 0 & 1 & 2 \\
\hline & -644 & -50 & 10 & 16 & 14 & 10 & 9 & 11 & 12 & 10 & -31 & -95 & -299 \\
\hline & -291 & -90 & -4 & 10 & 14 & 10 & 9 & 11 & 12 & 13 & -9 & -71 & ND \\
\hline & 89 & 52 & 30 & 23 & 14 & 10 & 9 & 11 & 12 & 19 & 32 & 44 & 74 \\
\hline \multirow[t]{6}{*}{$\overline{2 \mathrm{Y}}$} & -36 & -14 & -5 & -2 & -1 & -1 & -1 & -1 & -1 & -2 & -6 & -18 & -91 \\
\hline & -56 & -17 & -6 & -2 & -1 & -1 & -1 & -1 & -1 & -2 & -6 & -14 & -44 \\
\hline & 2 & 1 & 0 & 0 & 0 & 0 & -1 & 0 & 0 & 0 & 0 & 1 & 2 \\
\hline & ND & -65 & 12 & 22 & 18 & 15 & 13 & 14 & 17 & 13 & -27 & -138 & -418 \\
\hline & -373 & -129 & -13 & 18 & 18 & 15 & 13 & 14 & 17 & 18 & -1 & -107 & ND \\
\hline & 119 & 72 & 44 & 27 & 17 & 15 & 13 & 14 & 17 & 25 & 39 & 60 & 105 \\
\hline \multirow[t]{6}{*}{$\overline{3 \mathrm{Y}}$} & -64 & -18 & -11 & -3 & -1 & -1 & -1 & -1 & -1 & -3 & -10 & -27 & $\overline{-14} 1$ \\
\hline & -122 & -21 & -13 & -3 & -1 & -1 & -1 & -1 & -1 & -3 & -9 & -21 & -57 \\
\hline & 4 & 1 & 1 & 0 & -1 & -1 & -1 & -1 & 0 & 0 & 1 & 1 & 3 \\
\hline & ND & -43 & 6 & 35 & 28 & 22 & 19 & 20 & 25 & 17 & -62 & -208 & -534 \\
\hline & -638 & -154 & -68 & 27 & 28 & 22 & 19 & 20 & 2 & 27 & -8 & -159 & -2260 \\
\hline & 208 & 102 & 81 & 41 & 28 & 21 & 19 & 20 & 25 & 37 & 61 & 87 & 146 \\
\hline \multirow[t]{6}{*}{$\overline{5 Y}$} & -106 & -31 & -13 & -6 & -2 & -1 & -1 & -1 & -2 & -5 & -17 & -45 & -472 \\
\hline & -256 & -38 & -14 & -6 & -2 & -1 & -1 & -1 & -2 & -5 & -15 & -32 & -88 \\
\hline & 7 & 2 & 1 & 0 & -1 & -1 & -1 & -1 & -1 & 0 & 1 & 2 & 5 \\
\hline & ND & -41 & 53 & 64 & 49 & 39 & 32 & 35 & 41 & 25 & -116 & -334 & -753 \\
\hline & -1000 & -295 & -55 & 34 & 48 & 39 & 32 & 35 & 43 & 47 & -4 & -249 & ND \\
\hline & 377 & 183 & 119 & 80 & 49 & 38 & 32 & 34 & 43 & 60 & 99 & 140 & 233 \\
\hline \multirow[t]{6}{*}{$\overline{10 Y}$} & -172 & -69 & -30 & -10 & -4 & -3 & -2 & -2 & -4 & -9 & -35 & -103 & $\bar{N} \mathrm{D}$ \\
\hline & ND & -95 & -34 & -10 & -4 & -3 & -2 & -2 & -4 & -9 & -28 & -61 & -159 \\
\hline & 15 & 6 & 2 & 0 & -2 & -2 & -2 & -2 & -1 & 0 & 2 & 5 & 10 \\
\hline & ND & 40 & 158 & 146 & 109 & 82 & 67 & 70 & 80 & 32 & -271 & -625 & -1100 \\
\hline & -1531 & -762 & -232 & 75 & 103 & 82 & 67 & 70 & 85 & 95 & 16 & -781 & ND \\
\hline & 786 & 451 & 289 & 166 & 108 & 80 & 67 & 68 & 84 & 120 & 192 & 267 & 439 \\
\hline
\end{tabular}


Table 8. CEV model $(\beta=0.8, \nu=0.25)$ : errors in bps on the BS implied volatility using the 6 second order implied volatility approximations $\operatorname{AppImpVolLN}\left(2, x_{0}\right)$, $\operatorname{AppImpVolLN}(2, \mathrm{k}), \quad \operatorname{AppImpVolLN}\left(2, x_{\mathrm{av}}\right), \quad \operatorname{AppImpVolN}\left(2, S_{0}\right), \quad \operatorname{AppImpVolN}(2, \mathrm{~K}) \quad$ and AppImpVolN $\left(2, S_{\text {av }}\right)$.

\begin{tabular}{|c|c|c|c|c|c|c|c|c|c|c|c|c|c|}
\hline \multirow[t]{6}{*}{$3 \mathrm{M}$} & -1 & -1 & 0 & 0 & 0 & 0 & 0 & 0 & 0 & 0 & 0 & -1 & -1 \\
\hline & -1 & -1 & -1 & 0 & 0 & 0 & 0 & 0 & 0 & 0 & 0 & -1 & -1 \\
\hline & 0 & 0 & 0 & 0 & 0 & 0 & 0 & 0 & 0 & 0 & 0 & 0 & 0 \\
\hline & 27 & 18 & 12 & 7 & 4 & 2 & 2 & 2 & 3 & 5 & 11 & 15 & 19 \\
\hline & 29 & 19 & 12 & 7 & 4 & 2 & 2 & 2 & 3 & 5 & 11 & 15 & 18 \\
\hline & 21 & 14 & 9 & 6 & 3 & 2 & 2 & 2 & 3 & 4 & 9 & 11 & 15 \\
\hline \multirow[t]{6}{*}{$\overline{6 \mathrm{M}}$} & -2 & -1 & -1 & 0 & 0 & 0 & 0 & 0 & 0 & 0 & -1 & -1 & $\overline{-1}$ \\
\hline & -2 & -1 & -1 & 0 & 0 & 0 & 0 & 0 & 0 & 0 & -1 & -1 & -1 \\
\hline & 1 & 0 & 0 & 0 & 0 & 0 & 0 & 0 & 0 & 0 & 0 & 0 & 1 \\
\hline & 41 & 20 & 13 & 9 & 5 & 4 & 3 & 4 & 5 & 10 & 13 & 21 & 36 \\
\hline & 44 & 21 & 14 & 9 & 6 & 4 & 3 & 4 & 5 & 9 & 12 & 20 & 33 \\
\hline & 33 & 16 & 11 & 7 & 5 & 4 & 3 & 3 & 4 & 8 & 10 & 16 & 26 \\
\hline \multirow[t]{6}{*}{$\overline{1 Y}$} & -3 & -2 & -1 & -1 & 0 & 0 & 0 & 0 & 0 & -1 & -1 & -2 & -3 \\
\hline & -4 & -2 & -1 & -1 & 0 & 0 & 0 & 0 & 0 & -1 & -1 & -1 & -3 \\
\hline & 1 & 1 & 0 & 0 & 0 & 0 & 0 & 0 & 0 & 0 & 0 & 0 & 1 \\
\hline & 79 & 44 & 24 & 17 & 9 & 7 & 6 & 7 & 10 & 16 & 28 & 39 & 74 \\
\hline & 88 & 48 & 25 & 17 & 9 & 7 & 6 & 7 & 10 & 15 & 27 & 36 & 67 \\
\hline & 64 & 36 & 20 & 14 & 8 & 7 & 6 & 7 & 9 & 13 & 22 & 29 & 54 \\
\hline \multirow[t]{6}{*}{$1.5 \mathrm{Y}$} & -4 & -3 & -1 & -1 & -1 & 0 & 0 & 0 & -1 & -1 & -2 & -2 & $\overline{-4}$ \\
\hline & -5 & -3 & -2 & -1 & -1 & 0 & 0 & 0 & -1 & -1 & -2 & -2 & -4 \\
\hline & 2 & 1 & 0 & 0 & 0 & 0 & 0 & 0 & 0 & 0 & 0 & 1 & 2 \\
\hline & 108 & 64 & 36 & 27 & 15 & 10 & 9 & 11 & 13 & 23 & 42 & 59 & 104 \\
\hline & 123 & 69 & 38 & 28 & 15 & 10 & 9 & 11 & 13 & 22 & 39 & 54 & 91 \\
\hline & 89 & 52 & 30 & 23 & 14 & 10 & 9 & 11 & 12 & 19 & 32 & 44 & 74 \\
\hline \multirow[t]{6}{*}{$2 \mathrm{Y}$} & -6 & -4 & -2 & -1 & -1 & -1 & -1 & -1 & -1 & -1 & -2 & -3 & -6 \\
\hline & -7 & -4 & -2 & -1 & -1 & -1 & -1 & -1 & -1 & -1 & -2 & -3 & -5 \\
\hline & 2 & 1 & 0 & 0 & 0 & 0 & -1 & 0 & 0 & 0 & 0 & 1 & 2 \\
\hline & 145 & 87 & 52 & 31 & 19 & 15 & 13 & 14 & 19 & 30 & 50 & 80 & 149 \\
\hline & 167 & 97 & 55 & 32 & 19 & 15 & 13 & 14 & 18 & 29 & 47 & 72 & 127 \\
\hline & 119 & 72 & 44 & 27 & 17 & 15 & 13 & 14 & 17 & 25 & 39 & 60 & 105 \\
\hline \multirow[t]{6}{*}{$3 \mathrm{Y}$} & -10 & -5 & -4 & -2 & -1 & -1 & -1 & -1 & -1 & -2 & -3 & -5 & -9 \\
\hline & -12 & -6 & -4 & -2 & -1 & -1 & -1 & -1 & -1 & -2 & -3 & -4 & -7 \\
\hline & 4 & 1 & 1 & 0 & -1 & -1 & -1 & -1 & 0 & 0 & 1 & 1 & 3 \\
\hline & 249 & 121 & 96 & 47 & 31 & 22 & 19 & 20 & 28 & 45 & 80 & 120 & 212 \\
\hline & 301 & 136 & 105 & 49 & 31 & 22 & 19 & 20 & 28 & 43 & 73 & 106 & 176 \\
\hline & 208 & 102 & 81 & 41 & 28 & 21 & 19 & 20 & 25 & 37 & 61 & 87 & 146 \\
\hline \multirow[t]{6}{*}{$5 \mathrm{Y}$} & -18 & -9 & -5 & -4 & -2 & -1 & -1 & -1 & -2 & -3 & -5 & -8 & -14 \\
\hline & -23 & -10 & -6 & -4 & -2 & -1 & -1 & -1 & -2 & -3 & -5 & -7 & -11 \\
\hline & 7 & 2 & 1 & 0 & -1 & -1 & -1 & -1 & -1 & 0 & 1 & 2 & 5 \\
\hline & 443 & 216 & 140 & 92 & 52 & 39 & 32 & 35 & 48 & 74 & 132 & 196 & 352 \\
\hline & 571 & 252 & 155 & 98 & 53 & 40 & 32 & 35 & 47 & 69 & 117 & 167 & 277 \\
\hline & 377 & 183 & 119 & 80 & 49 & 38 & 32 & 34 & 43 & 60 & 99 & 140 & 233 \\
\hline \multirow[t]{6}{*}{$\overline{10 Y}$} & -33 & -19 & -12 & -7 & -4 & -3 & -2 & -2 & -3 & -6 & -10 & -16 & -29 \\
\hline & -47 & -24 & -14 & -7 & -4 & -3 & -2 & -2 & -3 & -5 & -9 & -12 & -21 \\
\hline & 15 & 6 & 2 & 0 & -2 & -2 & -2 & -2 & -1 & 0 & 2 & 5 & 10 \\
\hline & 904 & 522 & 333 & 188 & 117 & 82 & 67 & 70 & 94 & 149 & 264 & 394 & 725 \\
\hline & 1289 & 660 & 390 & 203 & 120 & 83 & 67 & 70 & 91 & 137 & 224 & 313 & 510 \\
\hline & 786 & 451 & 289 & 166 & 108 & 80 & 67 & 68 & 84 & 120 & 192 & 267 & 439 \\
\hline
\end{tabular}


Table 9. CEV model $(\beta=0.8, \nu=0.25)$ : errors in bps on the BS implied volatility using the 6 third order price approximations ImpVol (AppPriceLN $\left.\left(3, x_{0}\right)\right), \operatorname{ImpVol}(\operatorname{AppPriceLN}(3, \mathrm{k})), \operatorname{ImpVol}\left(\operatorname{AppPriceLN}\left(3, x_{\mathrm{av}}\right)\right)$, ImpVol (AppPriceN $\left(3, S_{0}\right)$ ), ImpVol (AppPriceN $\left.(3, \mathrm{~K})\right)$ and ImpVol (AppPriceN $\left(3, S_{\mathrm{av}}\right)$ ).

\begin{tabular}{|c|c|c|c|c|c|c|c|c|c|c|c|c|c|}
\hline \multirow[t]{6}{*}{$3 \mathrm{M}$} & -1 & 0 & 0 & 0 & 0 & 0 & 0 & 0 & 0 & 0 & 0 & 0 & 0 \\
\hline & 1 & 0 & 0 & 0 & 0 & 0 & 0 & 0 & 0 & 0 & 0 & 0 & 0 \\
\hline & 0 & 0 & 0 & 0 & 0 & 0 & 0 & 0 & 0 & 0 & 0 & 0 & 0 \\
\hline & 6 & -4 & -2 & -1 & 0 & 0 & 0 & 0 & 0 & 0 & 2 & 0 & -6 \\
\hline & -22 & -1 & 2 & 1 & 0 & 0 & 0 & 0 & 0 & 0 & -2 & -3 & -2 \\
\hline & -1 & 0 & 0 & 0 & 0 & 0 & 0 & 0 & 0 & 0 & 0 & 0 & 0 \\
\hline \multirow[t]{6}{*}{$\overline{6 \mathrm{M}}$} & -1 & 0 & 0 & 0 & 0 & 0 & 0 & 0 & 0 & 0 & 0 & 0 & $\overline{1}$ \\
\hline & 1 & 0 & 0 & 0 & 0 & 0 & 0 & 0 & 0 & 0 & 0 & 0 & -1 \\
\hline & 0 & 0 & 0 & 0 & 0 & 0 & 0 & 0 & 0 & 0 & 0 & 0 & 0 \\
\hline & -17 & -4 & -1 & 0 & 0 & 0 & 0 & 0 & 0 & 1 & 2 & 4 & -12 \\
\hline & -13 & 4 & 2 & 0 & 0 & 0 & 0 & 0 & 0 & -1 & -1 & -5 & -12 \\
\hline & -1 & 0 & 0 & 0 & 0 & 0 & 0 & 0 & 0 & 0 & 0 & 0 & -1 \\
\hline \multirow[t]{6}{*}{$\overline{1 Y}$} & 1 & 1 & 0 & 0 & 0 & 0 & 0 & 0 & 0 & 0 & 0 & 0 & 1 \\
\hline & -2 & 0 & 0 & 0 & 0 & 0 & 0 & 0 & 0 & 0 & 0 & 0 & 3 \\
\hline & 0 & 0 & 0 & 0 & 0 & 0 & 0 & 0 & 0 & 0 & 0 & 0 & 0 \\
\hline & -62 & -15 & -2 & -1 & 0 & 0 & 0 & 0 & 0 & 1 & 6 & 9 & -56 \\
\hline & -34 & 13 & 3 & 1 & 0 & 0 & 0 & 0 & 0 & -1 & -5 & -12 & -45 \\
\hline & -3 & 0 & 0 & 0 & 0 & 0 & 0 & 0 & 0 & 0 & 0 & 0 & -3 \\
\hline \multirow[t]{6}{*}{$1.5 \mathrm{Y}$} & -2 & 0 & 0 & 0 & 0 & 0 & 0 & 0 & 0 & 0 & 0 & 0 & 4 \\
\hline & 3 & 0 & 0 & 0 & 0 & 0 & 0 & 0 & 0 & 0 & 0 & 0 & -3 \\
\hline & 0 & 0 & 0 & 0 & 0 & 0 & 0 & 0 & 0 & 0 & 0 & 0 & 0 \\
\hline & -100 & -23 & -4 & -2 & 0 & 0 & 0 & 0 & 0 & 2 & 11 & 14 & -83 \\
\hline & -36 & 21 & 7 & 3 & 0 & 0 & 0 & 0 & 0 & -1 & -8 & -23 & -98 \\
\hline & -4 & -1 & 0 & 0 & 0 & 0 & 0 & 0 & 0 & 0 & 0 & -1 & -5 \\
\hline \multirow{6}{*}{$\overline{2 Y}$} & -3 & -1 & 0 & 0 & 0 & 0 & 0 & 0 & 0 & 0 & 0 & 1 & 10 \\
\hline & 4 & 1 & 0 & 0 & 0 & 0 & 0 & 0 & 0 & 0 & 0 & -1 & -7 \\
\hline & 0 & 0 & 0 & 0 & 0 & 0 & 0 & 0 & 0 & 0 & 0 & 0 & 0 \\
\hline & -163 & -36 & -8 & -1 & 0 & 0 & 0 & 0 & 0 & 3 & 14 & 17 & -177 \\
\hline & -63 & 31 & 14 & 2 & 0 & 0 & 0 & 0 & 0 & -2 & -9 & -38 & -24 \\
\hline & -7 & -1 & 0 & 0 & 0 & 0 & 0 & 0 & 0 & 0 & 0 & -1 & -12 \\
\hline \multirow[t]{6}{*}{$\overline{3 Y}$} & -9 & -1 & 0 & 0 & 0 & 0 & 0 & 0 & 0 & 0 & 0 & 1 & 16 \\
\hline & 11 & 1 & 0 & 0 & 0 & 0 & 0 & 0 & 0 & 0 & 0 & -1 & -10 \\
\hline & 0 & 0 & 0 & 0 & 0 & 0 & 0 & 0 & 0 & 0 & 0 & 0 & 0 \\
\hline & -818 & -48 & -23 & -2 & -1 & 0 & 0 & 0 & 1 & 5 & 29 & 16 & -27 \\
\hline & -250 & 52 & 41 & 4 & 1 & 0 & 0 & 0 & -1 & -3 & -19 & -69 & ND \\
\hline & -24 & -2 & -1 & 0 & 0 & 0 & 0 & 0 & 0 & 0 & -1 & -3 & -23 \\
\hline \multirow[t]{6}{*}{$5 \mathrm{Y}$} & -18 & -1 & 0 & 0 & 0 & 0 & 0 & 0 & 0 & 0 & 1 & 2 & 39 \\
\hline & 23 & 1 & 0 & 0 & 0 & 0 & 0 & 0 & 0 & 0 & -1 & -2 & -21 \\
\hline & 0 & 0 & 0 & 0 & 0 & 0 & 0 & 0 & 0 & 0 & 0 & 0 & 0 \\
\hline & ND & -103 & -26 & -7 & -2 & -1 & 0 & 0 & 1 & 12 & 57 & -10 & -52 \\
\hline & -597 & 90 & 65 & 17 & 1 & 1 & 0 & -1 & -2 & -5 & -38 & -143 & ND \\
\hline & -73 & -5 & -1 & -1 & 0 & 0 & 0 & 0 & 0 & 0 & -2 & -7 & -76 \\
\hline \multirow[t]{6}{*}{$\overline{10 Y}$} & -34 & -5 & -1 & 0 & 0 & 0 & 0 & 0 & 0 & 0 & 1 & 7 & 147 \\
\hline & 28 & 3 & 1 & 0 & 0 & 0 & 0 & 0 & 0 & 0 & -1 & -7 & -59 \\
\hline & 0 & 0 & 0 & 0 & 0 & 0 & 0 & 0 & 0 & 0 & 0 & 0 & 0 \\
\hline & ND & -340 & -83 & -16 & -6 & -4 & -1 & 1 & 4 & 42 & 109 & -222 & -987 \\
\hline & -1200 & -42 & 230 & 49 & 5 & 1 & -1 & -3 & -4 & -13 & -94 & -588 & \\
\hline & -214 & -29 & -7 & -3 & -2 & -1 & -1 & -1 & -1 & -1 & -6 & -28 & ND \\
\hline
\end{tabular}


Table 10. CEV model $(\beta=0.8, \nu=0.25)$ : errors in bps on the BS implied volatility using the 6 third order implied volatility approximations $\operatorname{AppImpVolLN}\left(3, x_{0}\right), \operatorname{AppImpVolLN}(3, \mathrm{k})$, $\operatorname{AppImpVolLN}\left(3, x_{\text {av }}\right), \operatorname{AppImpVolN}(3, \mathrm{~S} 0), \operatorname{AppImpVolN}(3, \mathrm{~K})$ and $\operatorname{AppImpVolN}\left(3, S_{\text {av }}\right)$.

\begin{tabular}{|c|c|c|c|c|c|c|c|c|c|c|c|c|c|}
\hline \multirow[t]{6}{*}{$3 \mathrm{M}$} & 0 & 0 & 0 & 0 & 0 & 0 & 0 & 0 & 0 & 0 & 0 & 0 & 0 \\
\hline & 0 & 0 & 0 & 0 & 0 & 0 & 0 & 0 & 0 & 0 & 0 & 0 & 0 \\
\hline & 0 & 0 & 0 & 0 & 0 & 0 & 0 & 0 & 0 & 0 & 0 & 0 & 0 \\
\hline & 4 & 2 & 1 & 0 & 0 & 0 & 0 & 0 & 0 & 0 & -1 & -2 & -3 \\
\hline & -5 & -3 & -1 & 0 & 0 & 0 & 0 & 0 & 0 & 0 & 1 & 1 & 2 \\
\hline & 0 & 0 & 0 & 0 & 0 & 0 & 0 & 0 & 0 & 0 & 0 & 0 & 0 \\
\hline \multirow[t]{6}{*}{$\overline{6 \mathrm{M}}$} & 0 & 0 & 0 & 0 & 0 & 0 & 0 & 0 & 0 & 0 & 0 & 0 & 0 \\
\hline & 0 & 0 & 0 & 0 & 0 & 0 & 0 & 0 & 0 & 0 & 0 & 0 & 0 \\
\hline & 0 & 0 & 0 & 0 & 0 & 0 & 0 & 0 & 0 & 0 & 0 & 0 & 0 \\
\hline & 7 & 2 & 1 & 0 & 0 & 0 & 0 & 0 & 0 & -1 & -1 & -3 & -8 \\
\hline & -10 & -3 & -1 & 0 & 0 & 0 & 0 & 0 & 0 & 0 & 1 & 2 & 5 \\
\hline & 0 & 0 & 0 & 0 & 0 & 0 & 0 & 0 & 0 & 0 & 0 & 0 & 0 \\
\hline \multirow[t]{6}{*}{$1 \mathrm{Y}$} & 0 & 0 & 0 & 0 & 0 & 0 & 0 & 0 & 0 & 0 & 0 & 0 & 0 \\
\hline & 0 & 0 & 0 & 0 & 0 & 0 & 0 & 0 & 0 & 0 & 0 & 0 & 0 \\
\hline & 0 & 0 & 0 & 0 & 0 & 0 & 0 & 0 & 0 & 0 & 0 & 0 & 0 \\
\hline & 17 & 6 & 2 & 1 & 0 & 0 & 0 & 0 & 0 & -1 & -4 & -7 & -25 \\
\hline & -30 & -10 & -2 & -1 & 0 & 0 & 0 & 0 & 0 & 1 & 3 & 5 & 14 \\
\hline & 1 & 0 & 0 & 0 & 0 & 0 & 0 & 0 & 0 & 0 & 0 & 0 & 1 \\
\hline \multirow[t]{6}{*}{$1.5 \mathrm{Y}$} & 0 & 0 & 0 & 0 & 0 & 0 & 0 & 0 & 0 & 0 & 0 & 0 & 0 \\
\hline & 0 & 0 & 0 & 0 & 0 & 0 & 0 & 0 & 0 & 0 & 0 & 0 & 0 \\
\hline & 0 & 0 & 0 & 0 & 0 & 0 & 0 & 0 & 0 & 0 & 0 & 0 & 0 \\
\hline & 25 & 10 & 3 & 2 & 0 & 0 & 0 & 0 & 0 & -2 & -7 & -15 & -43 \\
\hline & -49 & -17 & -5 & -2 & 0 & 0 & 0 & 0 & 0 & 1 & 5 & 9 & 22 \\
\hline & 2 & 1 & 0 & 0 & 0 & 0 & 0 & 0 & 0 & 0 & 0 & 1 & 2 \\
\hline \multirow[t]{6}{*}{$\overline{2 \mathrm{Y}}$} & 0 & 0 & 0 & 0 & 0 & 0 & 0 & 0 & 0 & 0 & 0 & 0 & $\overline{0}$ \\
\hline & 0 & 0 & 0 & 0 & 0 & 0 & 0 & 0 & 0 & 0 & 0 & 0 & 0 \\
\hline & 0 & 0 & 0 & 0 & 0 & 0 & 0 & 0 & 0 & 0 & 0 & 0 & 0 \\
\hline & 37 & 16 & 6 & 1 & 0 & 0 & 0 & 0 & 0 & -2 & -9 & -24 & -79 \\
\hline & -80 & -29 & -9 & -2 & 0 & 0 & 0 & 0 & 0 & 2 & 6 & 14 & 35 \\
\hline & 4 & 1 & 0 & 0 & 0 & 0 & 0 & 0 & 0 & 0 & 0 & 1 & 4 \\
\hline \multirow[t]{6}{*}{$\overline{3 Y}$} & 0 & 0 & 0 & 0 & 0 & 0 & 0 & 0 & 0 & 0 & 0 & 0 & $\overline{0}$ \\
\hline & 0 & 0 & 0 & 0 & 0 & 0 & 0 & 0 & 0 & 0 & 0 & 0 & 0 \\
\hline & 0 & 0 & 0 & 0 & 0 & 0 & 0 & 0 & 0 & 0 & 0 & 0 & 0 \\
\hline & 77 & 23 & 15 & 2 & 0 & 0 & 0 & 0 & -1 & -5 & -20 & -47 & -143 \\
\hline & -214 & -48 & -28 & -4 & 0 & 0 & 0 & 0 & 0 & 3 & 11 & 23 & 55 \\
\hline & 12 & 2 & 1 & 0 & 0 & 0 & 0 & 0 & 0 & 0 & 1 & 2 & 8 \\
\hline \multirow[t]{6}{*}{$\overline{5 Y}$} & -1 & 0 & 0 & 0 & 0 & 0 & 0 & 0 & 0 & 0 & 0 & 0 & 0 \\
\hline & 1 & 0 & 0 & 0 & 0 & 0 & 0 & 0 & 0 & 0 & 0 & 0 & 0 \\
\hline & 0 & 0 & 0 & 0 & 0 & 0 & 0 & 0 & 0 & 0 & 0 & 0 & 0 \\
\hline & 160 & 50 & 22 & 7 & 0 & -1 & 0 & 0 & -2 & -10 & -47 & -108 & -350 \\
\hline & -618 & -130 & -47 & -15 & -1 & 0 & 0 & -1 & 0 & 6 & 22 & 43 & 102 \\
\hline & 36 & 7 & 2 & 0 & 0 & 0 & 0 & 0 & 0 & 0 & 2 & 6 & 21 \\
\hline \multirow{6}{*}{$\overline{10 Y}$} & -1 & -1 & -1 & 0 & 0 & 0 & 0 & 0 & 0 & 0 & 1 & 1 & $\overline{1}$ \\
\hline & 2 & 1 & 1 & 0 & 0 & 0 & 0 & 0 & 0 & 0 & 0 & 0 & 0 \\
\hline & 0 & 0 & 0 & 0 & 0 & 0 & 0 & 0 & 0 & 0 & 0 & 0 & 0 \\
\hline & 362 & 155 & 68 & 16 & 0 & -3 & -1 & 1 & -4 & -34 & -153 & -368 & -130 \\
\hline & -2195 & -631 & -216 & -44 & -6 & 1 & -1 & -3 & 0 & 14 & 53 & 102 & 231 \\
\hline & 113 & 34 & 10 & 0 & -1 & -1 & -1 & -1 & -1 & 1 & 8 & 21 & 70 \\
\hline
\end{tabular}


Table 11. CEV model $(\beta=0.8, \nu=0.25)$ : errors in bps on the BS implied volatility using the 4 approximations Av. ImpVol (AppPriceLN $(3,).), \operatorname{Av} . \operatorname{AppImpVolLN}(3,),.(H F)$, and (HLF).

\begin{tabular}{|c|c|c|c|c|c|c|c|c|c|c|c|c|c|}
\hline \multirow[t]{4}{*}{$3 \mathrm{M}$} & 0 & 0 & 0 & 0 & 0 & 0 & 0 & 0 & 0 & 0 & 0 & 0 & 0 \\
\hline & 0 & 0 & 0 & 0 & 0 & 0 & 0 & 0 & 0 & 0 & 0 & 0 & 0 \\
\hline & 0 & 0 & 0 & 0 & 0 & 0 & 0 & 0 & 0 & 0 & 0 & 0 & 0 \\
\hline & 0 & 0 & 0 & 0 & 0 & 0 & 0 & 0 & 0 & 0 & 0 & 0 & 0 \\
\hline \multirow[t]{4}{*}{$\overline{6 \mathrm{M}}$} & 0 & 0 & 0 & 0 & 0 & 0 & 0 & 0 & 0 & 0 & 0 & 0 & $\overline{0}$ \\
\hline & 0 & 0 & 0 & 0 & 0 & 0 & 0 & 0 & 0 & 0 & 0 & 0 & 0 \\
\hline & 0 & 0 & 0 & 0 & 0 & 0 & 0 & 0 & 0 & 0 & 0 & 0 & 0 \\
\hline & 0 & 0 & 0 & 0 & 0 & 0 & 0 & 0 & 0 & 0 & 0 & 0 & 0 \\
\hline \multirow[t]{4}{*}{$\overline{1 Y}$} & 0 & 0 & 0 & 0 & 0 & 0 & 0 & 0 & 0 & 0 & 0 & 0 & 0 \\
\hline & 0 & 0 & 0 & 0 & 0 & 0 & 0 & 0 & 0 & 0 & 0 & 0 & 0 \\
\hline & -1 & 0 & 0 & 0 & 0 & 0 & 0 & 0 & 0 & 0 & 0 & 0 & -1 \\
\hline & 0 & 0 & 0 & 0 & 0 & 0 & 0 & 0 & 0 & 0 & 0 & 0 & 0 \\
\hline \multirow[t]{4}{*}{$1.5 \mathrm{Y}$} & 0 & 0 & 0 & 0 & 0 & 0 & 0 & 0 & 0 & 0 & 0 & 0 & 1 \\
\hline & 0 & 0 & 0 & 0 & 0 & 0 & 0 & 0 & 0 & 0 & 0 & 0 & 0 \\
\hline & -2 & -1 & 0 & 0 & 0 & 0 & 0 & 0 & 0 & 0 & 0 & 0 & -2 \\
\hline & 0 & 0 & 0 & 0 & 0 & 0 & 0 & 0 & 0 & 0 & 0 & 0 & 0 \\
\hline \multirow[t]{4}{*}{$2 \mathrm{Y}$} & 0 & 0 & 0 & 0 & 0 & 0 & 0 & 0 & 0 & 0 & 0 & 0 & 2 \\
\hline & 0 & 0 & 0 & 0 & 0 & 0 & 0 & 0 & 0 & 0 & 0 & 0 & 0 \\
\hline & -3 & -1 & 0 & 0 & 0 & 0 & 0 & 0 & 0 & 0 & 0 & -1 & -3 \\
\hline & 0 & 0 & 0 & 0 & 0 & 0 & 0 & 0 & 0 & 0 & 0 & 0 & 0 \\
\hline \multirow[t]{4}{*}{$3 \mathrm{Y}$} & 1 & 0 & 0 & 0 & 0 & 0 & 0 & 0 & 0 & 0 & 0 & 0 & 3 \\
\hline & 0 & 0 & 0 & 0 & 0 & 0 & 0 & 0 & 0 & 0 & 0 & 0 & 0 \\
\hline & -9 & -2 & -1 & 0 & 0 & 0 & 0 & 0 & 0 & 0 & -1 & -2 & -6 \\
\hline & 0 & 0 & 0 & 0 & 0 & 0 & 0 & 0 & 0 & 0 & 0 & 0 & 0 \\
\hline \multirow[t]{4}{*}{$5 \mathrm{Y}$} & 2 & 0 & 0 & 0 & 0 & 0 & 0 & 0 & 0 & 0 & 0 & 0 & 9 \\
\hline & 0 & 0 & 0 & 0 & 0 & 0 & 0 & 0 & 0 & 0 & 0 & 0 & 0 \\
\hline & -24 & -5 & -2 & 0 & 0 & 0 & 0 & 0 & 0 & 0 & -2 & -4 & -14 \\
\hline & 0 & 0 & 0 & 0 & 0 & 0 & 0 & 0 & 0 & 0 & 0 & 0 & 0 \\
\hline \multirow[t]{4}{*}{$10 \mathrm{Y}$} & -3 & -1 & 0 & 0 & 0 & 0 & 0 & 0 & 0 & 0 & 0 & 0 & 44 \\
\hline & 0 & 0 & 0 & 0 & 0 & 0 & 0 & 0 & 0 & 0 & 0 & 0 & 0 \\
\hline & -69 & -24 & -8 & -1 & 0 & 0 & 0 & 0 & 0 & -1 & -6 & -14 & -44 \\
\hline & 0 & 0 & 0 & 0 & 0 & 0 & 0 & 0 & 0 & 0 & 0 & 0 & 0 \\
\hline
\end{tabular}


Table 12. CEV model $(\beta=0.8, \nu=0.25)$ : average per categories of strikes of errors in bps on the BS implied volatility using the 6 approximations Av.ImpVol $(\operatorname{AppPriceLN}(3,)$.$) ,$ ImpVol (AppPriceln $\left(3, x_{\mathrm{av}}\right)$ ), Av.AppImpVolLN $(3,),. \operatorname{AppImpVolLN}\left(3, x_{\mathrm{av}}\right),(\mathrm{HF})$, and (HLF).

\begin{tabular}{|c|c|c|c|c|c|}
\hline & far ITM & ITM & ATM & OTM & far OTM \\
\hline \multirow[t]{6}{*}{$\overline{3 \mathrm{M}}$} & $6.7 \mathrm{E}-2$ & $1.4 \mathrm{E}-4$ & $-7.9 \mathrm{E}-6$ & $1.8 \mathrm{E}-4$ & $1.5 \mathrm{E}-2$ \\
\hline & $-2.4 \mathrm{E}-4$ & $-1.4 \mathrm{E}-5$ & $-1.3 \mathrm{E}-5$ & $-1.3 \mathrm{E}-5$ & $-9.9 \mathrm{E}-5$ \\
\hline & $4.3 \mathrm{E}-4$ & $9.1 \mathrm{E}-5$ & $-8.2 \mathrm{E}-6$ & $7.3 \mathrm{E}-5$ & $2.6 \mathrm{E}-4$ \\
\hline & $-3.4 \mathrm{E}-5$ & $-9.6 \mathrm{E}-6$ & $-1.3 \mathrm{E}-5$ & $-8.7 \mathrm{E}-6$ & $-1.7 \mathrm{E}-5$ \\
\hline & $-9.1 \mathrm{E}-2$ & $-8.6 \mathrm{E}-3$ & $2.0 \mathrm{E}-4$ & $-7.3 \mathrm{E}-3$ & $-4.8 \mathrm{E}-2$ \\
\hline & $-6.4 \mathrm{E}-5$ & $1.7 \mathrm{E}-4$ & $2.4 \mathrm{E}-4$ & $1.5 \mathrm{E}-4$ & $-9.8 \mathrm{E}-6$ \\
\hline \multirow[t]{6}{*}{$6 \mathrm{M}$} & $3.6 \mathrm{E}-2$ & $1.6 \mathrm{E}-4$ & $-4.3 \mathrm{E}-5$ & $1.5 \mathrm{E}-4$ & $3.2 \mathrm{E}-2$ \\
\hline & $-3.0 \mathrm{E}-4$ & $-4.3 \mathrm{E}-5$ & $-5.3 \mathrm{E}-5$ & $-3.6 \mathrm{E}-5$ & $-2.4 \mathrm{E}-4$ \\
\hline & $1.0 \mathrm{E}-3$ & $1.5 \mathrm{E}-4$ & $-4.3 \mathrm{E}-5$ & $1.4 \mathrm{E}-4$ & $7.8 \mathrm{E}-4$ \\
\hline & $-6.5 \mathrm{E}-5$ & $-4.3 \mathrm{E}-5$ & $-5.3 \mathrm{E}-5$ & $-3.5 \mathrm{E}-5$ & $-4.9 \mathrm{E}-5$ \\
\hline & $-1.6 \mathrm{E}-1$ & $-7.9 \mathrm{E}-3$ & $9.2 \mathrm{E}-4$ & $-8.6 \mathrm{E}-3$ & $-1.3 \mathrm{E}-1$ \\
\hline & $2.5 \mathrm{E}-4$ & $8.6 \mathrm{E}-4$ & $9.5 \mathrm{E}-4$ & $7.1 \mathrm{E}-4$ & $1.3 \mathrm{E}-4$ \\
\hline \multirow[t]{6}{*}{$\overline{1 Y}$} & $9.6 \mathrm{E}-2$ & $5.1 \mathrm{E}-4$ & $-1.9 \mathrm{E}-4$ & $4.4 \mathrm{E}-4$ & $1.9 \mathrm{E}-1$ \\
\hline & $-1.1 \mathrm{E}-3$ & $-1.8 \mathrm{E}-4$ & $-2.1 \mathrm{E}-4$ & $-1.4 \mathrm{E}-4$ & $-1.1 \mathrm{E}-3$ \\
\hline & $4.2 \mathrm{E}-3$ & $4.3 \mathrm{E}-4$ & $-1.9 \mathrm{E}-4$ & $5.5 \mathrm{E}-4$ & $3.0 \mathrm{E}-3$ \\
\hline & $-2.5 \mathrm{E}-4$ & $-1.8 \mathrm{E}-4$ & $-2.1 \mathrm{E}-4$ & $-1.4 \mathrm{E}-4$ & $-2.1 \mathrm{E}-4$ \\
\hline & $-6.3 \mathrm{E}-1$ & $-2.2 \mathrm{E}-2$ & $3.8 \mathrm{E}-3$ & $-3.6 \mathrm{E}-2$ & $-5.1 \mathrm{E}-1$ \\
\hline & $1.2 \mathrm{E}-3$ & $3.7 \mathrm{E}-3$ & $3.8 \mathrm{E}-3$ & $2.8 \mathrm{E}-3$ & $4.4 \mathrm{E}-4$ \\
\hline \multirow[t]{6}{*}{$1.5 \mathrm{Y}$} & $9.2 \mathrm{E}-2$ & $1.2 \mathrm{E}-3$ & $-3.9 \mathrm{E}-4$ & $7.5 \mathrm{E}-4$ & $2.8 \mathrm{E}-1$ \\
\hline & $-1.8 \mathrm{E}-3$ & $-4.0 \mathrm{E}-4$ & $-4.5 \mathrm{E}-4$ & $-3.2 \mathrm{E}-4$ & $-2.1 \mathrm{E}-3$ \\
\hline & $8.5 \mathrm{E}-3$ & $1.1 \mathrm{E}-3$ & $-3.9 \mathrm{E}-4$ & $1.1 \mathrm{E}-3$ & $6.1 \mathrm{E}-3$ \\
\hline & $-4.9 \mathrm{E}-4$ & $-3.9 \mathrm{E}-4$ & $-4.5 \mathrm{E}-4$ & $-3.0 \mathrm{E}-4$ & $-4.0 \mathrm{E}-4$ \\
\hline & $-1.2 \mathrm{E}+0$ & $-5.5 \mathrm{E}-2$ & $8.3 \mathrm{E}-3$ & $-7.3 \mathrm{E}-2$ & $-9.9 \mathrm{E}-1$ \\
\hline & $3.9 \mathrm{E}-3$ & $8.4 \mathrm{E}-3$ & $8.6 \mathrm{E}-3$ & $6.2 \mathrm{E}-3$ & $1.1 \mathrm{E}-3$ \\
\hline \multirow[t]{6}{*}{$2 \mathrm{Y}$} & $1.4 \mathrm{E}-1$ & $1.7 \mathrm{E}-3$ & $-6.4 \mathrm{E}-4$ & $1.4 \mathrm{E}-3$ & $8.7 \mathrm{E}-1$ \\
\hline & $-3.1 \mathrm{E}-3$ & $-7.1 \mathrm{E}-4$ & $-7.9 \mathrm{E}-4$ & $-5.3 \mathrm{E}-4$ & $-4.7 \mathrm{E}-3$ \\
\hline & $1.5 \mathrm{E}-2$ & $1.8 \mathrm{E}-3$ & $-6.4 \mathrm{E}-4$ & $1.7 \mathrm{E}-3$ & $1.1 \mathrm{E}-2$ \\
\hline & $-8.9 \mathrm{E}-4$ & $-7.1 \mathrm{E}-4$ & $-7.9 \mathrm{E}-4$ & $-5.1 \mathrm{E}-4$ & $-8.1 \mathrm{E}-4$ \\
\hline & $-2.0 \mathrm{E}+0$ & $-9.6 \mathrm{E}-2$ & $1.5 \mathrm{E}-2$ & $-1.0 \mathrm{E}-1$ & $-1.9 \mathrm{E}+0$ \\
\hline & $7.5 \mathrm{E}-3$ & $1.5 \mathrm{E}-2$ & $1.5 \mathrm{E}-2$ & $1.1 \mathrm{E}-2$ & $1.2 \mathrm{E}-3$ \\
\hline \multirow[t]{6}{*}{$3 \mathrm{Y}$} & $5.8 \mathrm{E}-1$ & $2.5 \mathrm{E}-3$ & $-1.5 \mathrm{E}-3$ & $1.9 \mathrm{E}-3$ & $1.5 \mathrm{E}+0$ \\
\hline & $-9.9 \mathrm{E}-3$ & $-1.6 \mathrm{E}-3$ & $-1.7 \mathrm{E}-3$ & $-1.1 \mathrm{E}-3$ & $-9.4 \mathrm{E}-3$ \\
\hline & $3.7 \mathrm{E}-2$ & $5.3 \mathrm{E}-3$ & $-1.5 \mathrm{E}-3$ & $3.8 \mathrm{E}-3$ & $2.2 \mathrm{E}-2$ \\
\hline & $-2.4 \mathrm{E}-3$ & $-1.5 \mathrm{E}-3$ & $-1.7 \mathrm{E}-3$ & $-1.1 \mathrm{E}-3$ & $-1.6 \mathrm{E}-3$ \\
\hline & $-5.2 \mathrm{E}+0$ & $-3.5 \mathrm{E}-1$ & $3.4 \mathrm{E}-2$ & $-2.6 \mathrm{E}-1$ & $-3.8 \mathrm{E}+0$ \\
\hline & $1.8 \mathrm{E}-2$ & $3.5 \mathrm{E}-2$ & $3.5 \mathrm{E}-2$ & $2.4 \mathrm{E}-2$ & $3.1 \mathrm{E}-3$ \\
\hline \multirow[t]{6}{*}{$\overline{5 Y}$} & $1.2 \mathrm{E}+0$ & $6.2 \mathrm{E}-3$ & $-3.6 \mathrm{E}-3$ & $1.2 \mathrm{E}-3$ & $4.5 \mathrm{E}+0$ \\
\hline & $-3.0 \mathrm{E}-2$ & $-4.0 \mathrm{E}-3$ & $-4.2 \mathrm{E}-3$ & $-2.7 \mathrm{E}-3$ & $-2.7 \mathrm{E}-2$ \\
\hline & $1.0 \mathrm{E}-1$ & $1.2 \mathrm{E}-2$ & $-3.5 \mathrm{E}-3$ & $9.4 \mathrm{E}-3$ & $5.4 \mathrm{E}-2$ \\
\hline & $-7.2 \mathrm{E}-3$ & $-3.9 \mathrm{E}-3$ & $-4.3 \mathrm{E}-3$ & $-2.5 \mathrm{E}-3$ & $-4.4 \mathrm{E}-3$ \\
\hline & $-1.5 \mathrm{E}+1$ & $-6.9 \mathrm{E}-1$ & $9.4 \mathrm{E}-2$ & $-6.7 \mathrm{E}-1$ & $-9.1 \mathrm{E}+0$ \\
\hline & $6.5 \mathrm{E}-2$ & $1.1 \mathrm{E}-1$ & $9.7 \mathrm{E}-2$ & $6.3 \mathrm{E}-2$ & $7.6 \mathrm{E}-3$ \\
\hline \multirow[t]{6}{*}{$\overline{10 Y}$} & $-2.1 \mathrm{E}+0$ & $-4.6 \mathrm{E}-2$ & $-1.1 \mathrm{E}-2$ & $-3.2 \mathrm{E}-2$ & $2.2 \mathrm{E}+1$ \\
\hline & $-9.7 \mathrm{E}-2$ & $-1.1 \mathrm{E}-2$ & $-1.2 \mathrm{E}-2$ & $-7.5 \mathrm{E}-3$ & $-1.3 \mathrm{E}-1$ \\
\hline & $2.8 \mathrm{E}-1$ & $3.8 \mathrm{E}-2$ & $-1.1 \mathrm{E}-2$ & $2.9 \mathrm{E}-2$ & $1.7 \mathrm{E}-1$ \\
\hline & $-2.3 \mathrm{E}-2$ & $-1.1 \mathrm{E}-2$ & $-1.3 \mathrm{E}-2$ & $-6.9 \mathrm{E}-3$ & $-1.8 \mathrm{E}-2$ \\
\hline & $-4.6 \mathrm{E}+1$ & $-3.1 \mathrm{E}+0$ & $3.8 \mathrm{E}-1$ & $-2.4 \mathrm{E}+0$ & $-2.9 \mathrm{E}+1$ \\
\hline & $4.4 \mathrm{E}-1$ & $4.8 \mathrm{E}-1$ & $4.0 \mathrm{E}-1$ & $2.3 \mathrm{E}-1$ & $2.2 \mathrm{E}-2$ \\
\hline
\end{tabular}


Table 13. CEV model $(\beta=0.2, \nu=0.25)$ : errors in bps on the BS implied volatility using the 6 approximations $\operatorname{ImpVol}\left(\operatorname{AppPriceLN}\left(3, x_{\mathrm{av}}\right)\right), \operatorname{AppImpVolLN}\left(3, x_{\mathrm{av}}\right)$, ImpVol (AppPriceN $\left(3, S_{\mathrm{av}}\right)$ ), AppImpVolN $\left(3, S_{\mathrm{av}}\right),(\mathrm{HF})$ and $(\mathrm{HLF})$.

\begin{tabular}{|c|c|c|c|c|c|c|c|c|c|c|c|c|c|}
\hline \multirow[t]{6}{*}{$3 \mathrm{M}$} & 0 & 0 & 0 & 0 & 0 & 0 & 0 & 0 & 0 & 0 & 0 & 0 & 0 \\
\hline & 0 & 0 & 0 & 0 & 0 & 0 & 0 & 0 & 0 & 0 & 0 & 0 & 0 \\
\hline & 0 & 0 & 0 & 0 & 0 & 0 & 0 & 0 & 0 & 0 & 0 & 0 & 0 \\
\hline & 0 & 0 & 0 & 0 & 0 & 0 & 0 & 0 & 0 & 0 & 0 & 0 & 0 \\
\hline & -1 & 0 & 0 & 0 & 0 & 0 & 0 & 0 & 0 & 0 & 0 & 0 & 0 \\
\hline & 0 & 0 & 0 & 0 & 0 & 0 & 0 & 0 & 0 & 0 & 0 & 0 & 0 \\
\hline \multirow[t]{6}{*}{$6 \mathrm{M}$} & 0 & 0 & 0 & 0 & 0 & 0 & 0 & 0 & 0 & 0 & 0 & 0 & 0 \\
\hline & 0 & 0 & 0 & 0 & 0 & 0 & 0 & 0 & 0 & 0 & 0 & 0 & 0 \\
\hline & 0 & 0 & 0 & 0 & 0 & 0 & 0 & 0 & 0 & 0 & 0 & 0 & 0 \\
\hline & 0 & 0 & 0 & 0 & 0 & 0 & 0 & 0 & 0 & 0 & 0 & 0 & 0 \\
\hline & -1 & 0 & 0 & 0 & 0 & 0 & 0 & 0 & 0 & 0 & 0 & 0 & -1 \\
\hline & 0 & 0 & 0 & 0 & 0 & 0 & 0 & 0 & 0 & 0 & 0 & 0 & 0 \\
\hline \multirow{6}{*}{$\overline{1 Y}$} & 0 & 0 & 0 & 0 & 0 & 0 & 0 & 0 & 0 & 0 & 0 & 0 & -1 \\
\hline & 0 & 0 & 0 & 0 & 0 & 0 & 0 & 0 & 0 & 0 & 0 & 0 & 0 \\
\hline & 0 & 0 & 0 & 0 & 0 & 0 & 0 & 0 & 0 & 0 & 0 & 0 & 0 \\
\hline & 1 & 0 & 0 & 0 & 0 & 0 & 0 & 0 & 0 & 0 & 0 & 0 & 0 \\
\hline & -5 & -1 & 0 & 0 & 0 & 0 & 0 & 0 & 0 & 0 & 0 & -1 & -2 \\
\hline & 0 & 0 & 0 & 0 & 0 & 0 & 0 & 0 & 0 & 0 & 0 & 0 & 0 \\
\hline \multirow[t]{6}{*}{$1.5 \mathrm{Y}$} & -1 & 0 & 0 & 0 & 0 & 0 & 0 & 0 & 0 & 0 & 0 & 0 & -1 \\
\hline & 0 & 0 & 0 & 0 & 0 & 0 & 0 & 0 & 0 & 0 & 0 & 0 & 0 \\
\hline & 1 & 0 & 0 & 0 & 0 & 0 & 0 & 0 & 0 & 0 & 0 & 0 & 0 \\
\hline & 1 & 1 & 0 & 0 & 0 & 0 & 0 & 0 & 0 & 0 & 0 & 0 & 0 \\
\hline & -9 & -3 & -1 & 0 & 0 & 0 & 0 & 0 & 0 & 0 & -1 & -1 & -4 \\
\hline & -1 & 0 & 0 & 0 & 0 & 0 & 0 & 0 & 0 & 0 & 0 & 0 & 0 \\
\hline \multirow[t]{6}{*}{$2 \mathrm{Y}$} & -1 & 0 & 0 & 0 & 0 & 0 & 0 & 0 & 0 & 0 & 0 & 0 & -3 \\
\hline & -1 & 0 & 0 & 0 & 0 & 0 & 0 & 0 & 0 & 0 & 0 & 0 & 0 \\
\hline & 2 & 1 & 0 & 0 & 0 & 0 & 0 & 0 & 0 & 0 & 0 & 0 & 0 \\
\hline & 2 & 1 & 1 & 0 & 0 & 0 & 0 & 0 & 0 & 0 & 0 & 0 & 1 \\
\hline & -15 & -5 & -1 & 0 & 0 & 0 & 0 & 0 & 0 & 0 & -1 & -3 & -8 \\
\hline & -1 & -1 & 0 & 0 & 0 & 0 & 0 & 0 & 0 & 0 & 0 & 0 & 0 \\
\hline \multirow[t]{6}{*}{$3 \mathrm{Y}$} & -4 & -1 & -1 & -1 & -1 & -1 & 0 & 0 & 0 & 0 & 0 & -1 & -8 \\
\hline & -2 & -1 & -1 & -1 & -1 & -1 & 0 & 0 & 0 & 0 & 0 & 0 & -1 \\
\hline & 7 & 2 & 2 & 1 & 0 & 0 & 0 & 0 & 0 & 0 & 0 & 0 & 0 \\
\hline & 8 & 3 & 2 & 1 & 0 & 0 & 0 & 0 & 0 & 0 & 0 & 1 & 1 \\
\hline & -45 & -9 & -5 & -1 & 0 & 0 & 0 & 0 & 0 & -1 & -2 & -5 & -15 \\
\hline & -4 & -1 & -1 & 0 & 0 & 0 & 0 & 0 & 0 & 0 & 0 & 0 & 0 \\
\hline \multirow[t]{6}{*}{$5 \mathrm{Y}$} & 2 & -1 & -2 & -2 & -2 & -2 & -1 & -1 & -1 & 0 & 0 & -2 & -37 \\
\hline & 7 & -1 & -2 & -2 & -2 & -2 & -1 & -1 & -1 & 0 & 0 & 0 & -1 \\
\hline & 47 & 13 & 6 & 4 & 2 & 1 & 1 & 1 & 1 & 1 & 1 & 1 & 0 \\
\hline & 50 & 13 & 7 & 4 & 2 & 1 & 1 & 1 & 1 & 1 & 1 & 1 & 3 \\
\hline & -117 & -26 & -9 & -3 & 0 & 0 & 0 & 0 & 0 & -1 & -6 & -12 & -31 \\
\hline & 4 & 0 & 0 & 0 & 0 & 0 & 0 & 0 & 0 & 0 & -1 & -1 & -1 \\
\hline \multirow[t]{6}{*}{$\overline{10 Y}$} & 148 & 84 & 41 & 12 & 2 & -2 & -3 & -2 & -2 & -1 & -2 & -13 & $\mathrm{~N} D$ \\
\hline & 159 & 85 & 41 & 12 & 2 & -2 & -3 & -2 & -2 & -1 & -1 & -1 & -5 \\
\hline & 530 & 221 & 109 & 45 & 21 & 11 & 6 & 3 & 2 & 2 & 2 & 2 & -8 \\
\hline & 541 & 224 & 111 & 45 & 21 & 11 & 6 & 3 & 2 & 2 & 2 & 3 & 6 \\
\hline & -112 & -6 & 18 & 18 & 12 & 8 & 4 & 2 & -1 & -5 & -17 & -33 & -73 \\
\hline & 271 & 123 & 65 & 29 & 14 & 8 & 4 & 2 & 0 & -1 & -1 & -1 & -1 \\
\hline
\end{tabular}


Table 14. CEV model $(\beta=0.2, \nu=0.25)$ : average per categories of strikes of errors in bps on the BS implied volatility using the 6 approximations ImpVol (AppPriceLN $\left(3, x_{\mathrm{av}}\right)$ ), $\operatorname{AppImpVolLN}\left(3, x_{\mathrm{av}}\right), \operatorname{ImpVol}\left(\operatorname{AppPriceN}\left(3, S_{\mathrm{av}}\right)\right), \operatorname{AppImpVolN}\left(3, S_{\mathrm{av}}\right),(\mathrm{HF})$ and $(\mathrm{HLF})$.

\begin{tabular}{|c|c|c|c|c|c|}
\hline & far ITM & ITM & ATM & OTM & far OTM \\
\hline \multirow[t]{6}{*}{$\overline{3 \mathrm{M}}$} & $-5.4 \mathrm{E}-2$ & $-4.0 \mathrm{E}-3$ & $-3.4 \mathrm{E}-3$ & $-3.3 \mathrm{E}-3$ & $-2.9 \mathrm{E}-2$ \\
\hline & $-9.3 \mathrm{E}-3$ & $-3.1 \mathrm{E}-3$ & $-3.4 \mathrm{E}-3$ & $-1.9 \mathrm{E}-3$ & $-4.1 \mathrm{E}-3$ \\
\hline & $2.5 \mathrm{E}-2$ & $6.3 \mathrm{E}-3$ & $1.8 \mathrm{E}-3$ & $3.7 \mathrm{E}-3$ & $8.9 \mathrm{E}-3$ \\
\hline & $3.9 \mathrm{E}-2$ & $7.2 \mathrm{E}-3$ & $1.8 \mathrm{E}-3$ & $4.5 \mathrm{E}-3$ & $1.6 \mathrm{E}-2$ \\
\hline & $-3.8 \mathrm{E}-1$ & $-3.9 \mathrm{E}-2$ & $7.9 \mathrm{E}-5$ & $-2.9 \mathrm{E}-2$ & $-1.6 \mathrm{E}-1$ \\
\hline & $-2.1 \mathrm{E}-2$ & $-4.6 \mathrm{E}-3$ & $3.2 \mathrm{E}-4$ & $-2.6 \mathrm{E}-3$ & $-7.9 \mathrm{E}-3$ \\
\hline \multirow[t]{6}{*}{$6 \mathrm{M}$} & $-6.9 \mathrm{E}-2$ & $-1.5 \mathrm{E}-2$ & $-1.4 \mathrm{E}-2$ & $-7.5 \mathrm{E}-3$ & $-7.4 \mathrm{E}-2$ \\
\hline & $-2.1 \mathrm{E}-2$ & $-1.4 \mathrm{E}-2$ & $-1.4 \mathrm{E}-2$ & $-7.2 \mathrm{E}-3$ & $-1.1 \mathrm{E}-2$ \\
\hline & $6.8 \mathrm{E}-2$ & $1.6 \mathrm{E}-2$ & $6.9 \mathrm{E}-3$ & $9.6 \mathrm{E}-3$ & $2.5 \mathrm{E}-2$ \\
\hline & $9.0 \mathrm{E}-2$ & $1.7 \mathrm{E}-2$ & $6.9 \mathrm{E}-3$ & $1.0 \mathrm{E}-2$ & $4.3 \mathrm{E}-2$ \\
\hline & $-7.2 \mathrm{E}-1$ & $-4.5 \mathrm{E}-2$ & $1.5 \mathrm{E}-3$ & $-3.9 \mathrm{E}-2$ & $-4.3 \mathrm{E}-1$ \\
\hline & $-5.6 \mathrm{E}-2$ & $-7.4 \mathrm{E}-3$ & $1.8 \mathrm{E}-3$ & $-5.4 \mathrm{E}-3$ & $-2.2 \mathrm{E}-2$ \\
\hline \multirow[t]{6}{*}{$\overline{1 Y}$} & $-2.5 \mathrm{E}-1$ & $-6.6 \mathrm{E}-2$ & $-5.5 \mathrm{E}-2$ & $-2.8 \mathrm{E}-2$ & $-3.8 \mathrm{E}-1$ \\
\hline & $-9.9 \mathrm{E}-2$ & $-6.6 \mathrm{E}-2$ & $-5.5 \mathrm{E}-2$ & $-2.6 \mathrm{E}-2$ & $-4.6 \mathrm{E}-2$ \\
\hline & $3.2 \mathrm{E}-1$ & $6.4 \mathrm{E}-2$ & $2.8 \mathrm{E}-2$ & $3.5 \mathrm{E}-2$ & $7.8 \mathrm{E}-2$ \\
\hline & $4.1 \mathrm{E}-1$ & $6.7 \mathrm{E}-2$ & $2.8 \mathrm{E}-2$ & $3.8 \mathrm{E}-2$ & $1.5 \mathrm{E}-1$ \\
\hline & $-3.0 \mathrm{E}+0$ & $-1.4 \mathrm{E}-1$ & $8.2 \mathrm{E}-3$ & $-1.5 \mathrm{E}-1$ & $-1.6 \mathrm{E}+0$ \\
\hline & $-2.6 \mathrm{E}-1$ & $-2.3 \mathrm{E}-2$ & $8.8 \mathrm{E}-3$ & $-1.9 \mathrm{E}-2$ & $-7.2 \mathrm{E}-2$ \\
\hline \multirow[t]{6}{*}{$1.5 \mathrm{Y}$} & $-4.4 \mathrm{E}-1$ & $-1.6 \mathrm{E}-1$ & $-1.2 \mathrm{E}-1$ & $-6.2 \mathrm{E}-2$ & $-7.4 \mathrm{E}-1$ \\
\hline & $-2.4 \mathrm{E}-1$ & $-1.6 \mathrm{E}-1$ & $-1.2 \mathrm{E}-1$ & $-5.6 \mathrm{E}-2$ & $-8.8 \mathrm{E}-2$ \\
\hline & $7.4 \mathrm{E}-1$ & $1.7 \mathrm{E}-1$ & $6.2 \mathrm{E}-2$ & $7.1 \mathrm{E}-2$ & $1.5 \mathrm{E}-1$ \\
\hline & $8.8 \mathrm{E}-1$ & $1.8 \mathrm{E}-1$ & $6.2 \mathrm{E}-2$ & $7.8 \mathrm{E}-2$ & $2.9 \mathrm{E}-1$ \\
\hline & $-5.6 \mathrm{E}+0$ & $-3.5 \mathrm{E}-1$ & $1.7 \mathrm{E}-2$ & $-3.0 \mathrm{E}-1$ & $-3.0 \mathrm{E}+0$ \\
\hline & $-5.6 \mathrm{E}-1$ & $-5.8 \mathrm{E}-2$ & $1.9 \mathrm{E}-2$ & $-3.5 \mathrm{E}-2$ & $-1.3 \mathrm{E}-1$ \\
\hline \multirow[t]{6}{*}{$\overline{2 Y}$} & $-8.0 \mathrm{E}-1$ & $-3.1 \mathrm{E}-1$ & $-2.2 \mathrm{E}-1$ & $-9.6 \mathrm{E}-2$ & $-1.9 \mathrm{E}+0$ \\
\hline & $-4.9 \mathrm{E}-1$ & $-3.1 \mathrm{E}-1$ & $-2.2 \mathrm{E}-1$ & $-9.0 \mathrm{E}-2$ & $-1.8 \mathrm{E}-1$ \\
\hline & $1.5 \mathrm{E}+0$ & $3.2 \mathrm{E}-1$ & $1.2 \mathrm{E}-1$ & $1.1 \mathrm{E}-1$ & $2.1 \mathrm{E}-1$ \\
\hline & $1.7 \mathrm{E}+0$ & $3.3 \mathrm{E}-1$ & $1.2 \mathrm{E}-1$ & $1.2 \mathrm{E}-1$ & $5.1 \mathrm{E}-1$ \\
\hline & $-1.0 \mathrm{E}+1$ & $-6.1 \mathrm{E}-1$ & $3.0 \mathrm{E}-2$ & $-4.3 \mathrm{E}-1$ & $-5.5 \mathrm{E}+0$ \\
\hline & $-1.1 \mathrm{E}+0$ & $-9.2 \mathrm{E}-2$ & $3.6 \mathrm{E}-2$ & $-5.5 \mathrm{E}-2$ & $-2.1 \mathrm{E}-1$ \\
\hline \multirow[t]{6}{*}{$\overline{3 Y}$} & $-2.3 \mathrm{E}+0$ & $-8.0 \mathrm{E}-1$ & $-4.8 \mathrm{E}-1$ & $-2.0 \mathrm{E}-1$ & $-4.3 \mathrm{E}+0$ \\
\hline & $-1.4 \mathrm{E}+0$ & $-8.0 \mathrm{E}-1$ & $-4.8 \mathrm{E}-1$ & $-1.7 \mathrm{E}-1$ & $-3.5 \mathrm{E}-1$ \\
\hline & $4.8 \mathrm{E}+0$ & $9.8 \mathrm{E}-1$ & $2.8 \mathrm{E}-1$ & $2.4 \mathrm{E}-1$ & $3.4 \mathrm{E}-1$ \\
\hline & $5.4 \mathrm{E}+0$ & $1.0 \mathrm{E}+0$ & $2.8 \mathrm{E}-1$ & $2.6 \mathrm{E}-1$ & $9.3 \mathrm{E}-1$ \\
\hline & $-2.7 \mathrm{E}+1$ & $-2.1 \mathrm{E}+0$ & $8.9 \mathrm{E}-2$ & $-1.0 \mathrm{E}+0$ & $-9.9 \mathrm{E}+0$ \\
\hline & $-2.7 \mathrm{E}+0$ & $-2.7 \mathrm{E}-1$ & $9.7 \mathrm{E}-2$ & $-1.1 \mathrm{E}-1$ & $-3.7 \mathrm{E}-1$ \\
\hline
\end{tabular}


Table 15. CEV model $(\beta=0.5, \nu=0.4)$ : errors in bps on the BS implied volatility using the 4 approximations ImpVol (AppPriceLN $\left.\left(3, x_{\mathrm{av}}\right)\right)$, AppImpVolLN $\left(3, x_{\mathrm{av}}\right),(\mathrm{HF})$ and $(\mathrm{HLF})$.

\begin{tabular}{|c|c|c|c|c|c|c|c|c|c|c|c|c|c|}
\hline \multirow[t]{4}{*}{$3 \mathrm{M}$} & 0 & 0 & 0 & 0 & 0 & 0 & 0 & 0 & 0 & 0 & 0 & 0 & 0 \\
\hline & 0 & 0 & 0 & 0 & 0 & 0 & 0 & 0 & 0 & 0 & 0 & 0 & 0 \\
\hline & -1 & 0 & 0 & 0 & 0 & 0 & 0 & 0 & 0 & 0 & 0 & 0 & 0 \\
\hline & 0 & 0 & 0 & 0 & 0 & 0 & 0 & 0 & 0 & 0 & 0 & 0 & 0 \\
\hline \multirow[t]{4}{*}{$6 \mathrm{M}$} & 0 & 0 & 0 & 0 & 0 & 0 & 0 & 0 & 0 & 0 & 0 & 0 & $\overline{0}$ \\
\hline & 0 & 0 & 0 & 0 & 0 & 0 & 0 & 0 & 0 & 0 & 0 & 0 & 0 \\
\hline & -1 & 0 & 0 & 0 & 0 & 0 & 0 & 0 & 0 & 0 & 0 & 0 & -1 \\
\hline & 0 & 0 & 0 & 0 & 0 & 0 & 0 & 0 & 0 & 0 & 0 & 0 & 0 \\
\hline \multirow[t]{4}{*}{$\overline{1 Y}$} & 0 & 0 & 0 & 0 & 0 & 0 & 0 & 0 & 0 & 0 & 0 & 0 & 0 \\
\hline & 0 & 0 & 0 & 0 & 0 & 0 & 0 & 0 & 0 & 0 & 0 & 0 & 0 \\
\hline & -4 & -1 & 0 & 0 & 0 & 0 & 0 & 0 & 0 & 0 & 0 & -1 & -3 \\
\hline & 0 & 0 & 0 & 0 & 0 & 0 & 0 & 0 & 0 & 0 & 0 & 0 & 0 \\
\hline \multirow[t]{4}{*}{$1.5 \mathrm{Y}$} & 0 & 0 & 0 & 0 & 0 & 0 & 0 & 0 & 0 & 0 & 0 & 0 & 0 \\
\hline & 0 & 0 & 0 & 0 & 0 & 0 & 0 & 0 & 0 & 0 & 0 & 0 & 0 \\
\hline & -8 & -2 & 0 & 0 & 0 & 0 & 0 & 0 & 0 & 0 & -1 & -2 & -5 \\
\hline & 0 & 0 & 1 & 1 & 0 & 0 & 0 & 0 & 0 & 0 & 0 & 0 & 0 \\
\hline \multirow[t]{4}{*}{$2 \mathrm{Y}$} & 0 & 0 & 0 & 0 & 0 & 0 & 0 & 0 & 0 & 0 & 0 & 0 & 0 \\
\hline & 0 & 0 & 0 & 0 & 0 & 0 & 0 & 0 & 0 & 0 & 0 & 0 & 0 \\
\hline & -13 & -4 & 0 & 1 & 1 & 1 & 1 & 1 & 1 & 0 & -1 & -3 & -10 \\
\hline & 1 & 1 & 1 & 1 & 1 & 1 & 1 & 1 & 1 & 0 & 0 & 0 & 0 \\
\hline \multirow[t]{4}{*}{$3 \mathrm{Y}$} & -1 & -1 & -1 & -1 & -1 & -1 & -1 & -1 & 0 & 0 & 0 & 0 & $\overline{0}$ \\
\hline & -1 & -1 & -1 & -1 & -1 & -1 & -1 & -1 & 0 & 0 & 0 & 0 & 0 \\
\hline & -37 & -5 & -2 & 2 & 2 & 2 & 2 & 2 & 1 & 0 & -2 & -6 & -19 \\
\hline & 3 & 3 & 3 & 3 & 2 & 2 & 2 & 2 & 1 & 1 & 0 & 0 & 0 \\
\hline \multirow[t]{4}{*}{$\overline{5 Y}$} & 6 & 1 & 0 & -1 & -1 & -1 & -1 & -1 & -1 & -1 & 0 & 0 & -1 \\
\hline & 6 & 1 & 0 & -1 & -1 & -1 & -1 & -1 & -1 & -1 & 0 & 0 & 0 \\
\hline & -88 & -9 & 4 & 7 & 8 & 7 & 6 & 5 & 3 & 1 & -5 & -14 & -43 \\
\hline & 25 & 15 & 12 & 10 & 8 & 7 & 6 & 5 & 3 & 2 & 1 & 0 & -1 \\
\hline \multirow[t]{4}{*}{$10 \mathrm{Y}$} & 92 & 61 & 40 & 22 & 13 & 8 & 4 & 2 & 1 & 0 & 0 & -1 & -8 \\
\hline & 91 & 61 & 40 & 22 & 13 & 8 & 4 & 2 & 1 & 0 & 0 & 0 & -1 \\
\hline & -58 & 54 & 76 & 69 & 54 & 42 & 31 & 23 & 15 & 4 & -17 & -44 & -118 \\
\hline & 286 & 173 & 120 & 79 & 56 & 42 & 31 & 23 & 16 & 9 & 4 & 1 & -2 \\
\hline
\end{tabular}


Table 16. CEV model $(\beta=0.5, \nu=0.4)$ : average per categories of strikes of errors in bps on the BS implied volatility using the 4 approximations $\operatorname{ImpVol}\left(\operatorname{AppPriceLN}\left(3, x_{\mathrm{av}}\right)\right)$, $\operatorname{AppImpVolLN}\left(3, x_{\mathrm{av}}\right),(\mathrm{HF})$ and (HLF).

\begin{tabular}{lrrrrr}
\hline & far ITM & \multicolumn{1}{c}{ ITM } & ATM & OTM & far OTM \\
\hline $3 \mathrm{M}$ & $-6.9 \mathrm{E}-3$ & $-5.3 \mathrm{E}-3$ & $-5.4 \mathrm{E}-3$ & $-3.6 \mathrm{E}-3$ & $-3.2 \mathrm{E}-3$ \\
& $-4.6 \mathrm{E}-3$ & $-5.3 \mathrm{E}-3$ & $-5.4 \mathrm{E}-3$ & $-3.6 \mathrm{E}-3$ & $-2.2 \mathrm{E}-3$ \\
& $-3.8 \mathrm{E}-1$ & $-2.9 \mathrm{E}-2$ & $1.1 \mathrm{E}-2$ & $-2.4 \mathrm{E}-2$ & $-1.8 \mathrm{E}-1$ \\
& $-5.9 \mathrm{E}-3$ & $8.0 \mathrm{E}-3$ & $1.1 \mathrm{E}-2$ & $5.4 \mathrm{E}-3$ & $-3.0 \mathrm{E}-3$ \\
\hline $6 \mathrm{M}$ & $-2.3 \mathrm{E}-2$ & $-2.3 \mathrm{E}-2$ & $-2.1 \mathrm{E}-2$ & $-1.5 \mathrm{E}-2$ & $-1.1 \mathrm{E}-2$ \\
& $-2.1 \mathrm{E}-2$ & $-2.3 \mathrm{E}-2$ & $-2.1 \mathrm{E}-2$ & $-1.5 \mathrm{E}-2$ & $-8.3 \mathrm{E}-3$ \\
& $-6.8 \mathrm{E}-1$ & $4.2 \mathrm{E}-3$ & $4.4 \mathrm{E}-2$ & $-1.1 \mathrm{E}-2$ & $-4.8 \mathrm{E}-1$ \\
& $1.1 \mathrm{E}-2$ & $4.4 \mathrm{E}-2$ & $4.5 \mathrm{E}-2$ & $2.8 \mathrm{E}-2$ & $-3.4 \mathrm{E}-3$ \\
\hline $1 \mathrm{Y}$ & $-9.9 \mathrm{E}-2$ & $-9.5 \mathrm{E}-2$ & $-8.2 \mathrm{E}-2$ & $-5.3 \mathrm{E}-2$ & $-4.2 \mathrm{E}-2$ \\
& $-9.5 \mathrm{E}-2$ & $-9.4 \mathrm{E}-2$ & $-8.2 \mathrm{E}-2$ & $-5.3 \mathrm{E}-2$ & $-2.8 \mathrm{E}-2$ \\
& $-2.6 \mathrm{E}+0$ & $8.3 \mathrm{E}-2$ & $1.8 \mathrm{E}-1$ & $-5.3 \mathrm{E}-2$ & $-1.8 \mathrm{E}+0$ \\
& $8.7 \mathrm{E}-2$ & $2.0 \mathrm{E}-1$ & $1.8 \mathrm{E}-1$ & $1.0 \mathrm{E}-1$ & $-1.5 \mathrm{E}-2$ \\
\hline $1.5 \mathrm{Y}$ & $-2.3 \mathrm{E}-1$ & $-2.1 \mathrm{E}-1$ & $-1.7 \mathrm{E}-1$ & $-1.1 \mathrm{E}-1$ & $-7.9 \mathrm{E}-2$ \\
& $-2.2 \mathrm{E}-1$ & $-2.1 \mathrm{E}-1$ & $-1.7 \mathrm{E}-1$ & $-1.1 \mathrm{E}-1$ & $-5.2 \mathrm{E}-2$ \\
& $-4.7 \mathrm{E}+0$ & $2.0 \mathrm{E}-1$ & $4.1 \mathrm{E}-1$ & $-7.7 \mathrm{E}-2$ & $-3.5 \mathrm{E}+0$ \\
& $3.4 \mathrm{E}-1$ & $5.0 \mathrm{E}-1$ & $4.1 \mathrm{E}-1$ & $2.4 \mathrm{E}-1$ & $-2.5 \mathrm{E}-2$ \\
\hline $2 \mathrm{Y}$ & $-4.0 \mathrm{E}-1$ & $-3.6 \mathrm{E}-1$ & $-2.9 \mathrm{E}-1$ & $-1.8 \mathrm{E}-1$ & $-1.5 \mathrm{E}-1$ \\
& $-3.9 \mathrm{E}-1$ & $-3.6 \mathrm{E}-1$ & $-2.9 \mathrm{E}-1$ & $-1.8 \mathrm{E}-1$ & $-8.1 \mathrm{E}-2$ \\
& $-8.2 \mathrm{E}+0$ & $4.3 \mathrm{E}-1$ & $7.6 \mathrm{E}-1$ & $-3.7 \mathrm{E}-2$ & $-6.7 \mathrm{E}+0$ \\
& $8.1 \mathrm{E}-1$ & $9.7 \mathrm{E}-1$ & $7.7 \mathrm{E}-1$ & $4.2 \mathrm{E}-1$ & $-6.8 \mathrm{E}-2$ \\
\hline & $-7.0 \mathrm{E}-1$ & $-7.2 \mathrm{E}-1$ & $-5.7 \mathrm{E}-1$ & $-3.3 \mathrm{E}-1$ & $-3.0 \mathrm{E}-1$ \\
& $-6.6 \mathrm{E}-1$ & $-7.2 \mathrm{E}-1$ & $-5.8 \mathrm{E}-1$ & $-3.3 \mathrm{E}-1$ & $-1.4 \mathrm{E}-1$ \\
& $-2.1 \mathrm{E}+1$ & $7.9 \mathrm{E}-1$ & $1.8 \mathrm{E}+0$ & $-2.2 \mathrm{E}-1$ & $-1.3 \mathrm{E}+1$ \\
& $3.0 \mathrm{E}+0$ & $2.6 \mathrm{E}+0$ & $1.8 \mathrm{E}+0$ & $8.8 \mathrm{E}-1$ & $-1.2 \mathrm{E}-1$ \\
\hline
\end{tabular}


Table 17. CEV model $(\beta=0.8, \nu=0.25)$ : errors in bps on the deltas using the 6 approximations AppDeltaLN $\left(1, x_{0}\right), \operatorname{AppDeltaLN}(1, \mathrm{k}), \operatorname{AppDeltaLN}\left(1, x_{\mathrm{av}}\right), \operatorname{AppDeltaLN}\left(2, x_{0}\right)$, $\operatorname{AppDeltaLN}(2, \mathrm{k})$ and $\operatorname{AppDeltaLN}\left(2, x_{\mathrm{av}}\right)$.

\begin{tabular}{|c|c|c|c|c|c|c|c|c|c|c|c|c|c|}
\hline \multirow[t]{6}{*}{$3 \mathrm{M}$} & 1 & 2 & 2 & 1 & 1 & 0 & 0 & 0 & -1 & -1 & -2 & -2 & -2 \\
\hline & 1 & 0 & 0 & -1 & -1 & 0 & 0 & 0 & 0 & 1 & 0 & 0 & -1 \\
\hline & 0 & 0 & 0 & 0 & 0 & 0 & 0 & 0 & 0 & 0 & 0 & 0 & 0 \\
\hline & 0 & 0 & 0 & 0 & 0 & 0 & 0 & 0 & 0 & 0 & 0 & 0 & 0 \\
\hline & 0 & 0 & 0 & 0 & 0 & 0 & 0 & 0 & 0 & 0 & 0 & 0 & 0 \\
\hline & 0 & 0 & 0 & 0 & 0 & 0 & 0 & 0 & 0 & 0 & 0 & 0 & 0 \\
\hline \multirow[t]{6}{*}{$6 \mathrm{M}$} & 3 & 3 & 2 & 1 & 1 & 1 & 0 & -1 & -1 & -2 & -3 & -4 & $\overline{-4}$ \\
\hline & 1 & -1 & -1 & -1 & -1 & 0 & 0 & 0 & 1 & 1 & 1 & 0 & -1 \\
\hline & 0 & 0 & 0 & 0 & 0 & 0 & 0 & 0 & 0 & 0 & 0 & 0 & 0 \\
\hline & 0 & 0 & 0 & 0 & 0 & 0 & 0 & 0 & 0 & 0 & 0 & 0 & 0 \\
\hline & 0 & 0 & 0 & 0 & 0 & 0 & 0 & 0 & 0 & 0 & 0 & 0 & 0 \\
\hline & 0 & 0 & 0 & 0 & 0 & 0 & 0 & 0 & 0 & 0 & 0 & 0 & 0 \\
\hline \multirow[t]{6}{*}{$\overline{1 Y}$} & 5 & 5 & 3 & 3 & 1 & 1 & 0 & -1 & -2 & -4 & -6 & -8 & -9 \\
\hline & 1 & -2 & -3 & -2 & -1 & 0 & 0 & 0 & 1 & 2 & 2 & 1 & -3 \\
\hline & 0 & 0 & 0 & 1 & 0 & 0 & 0 & 0 & -1 & -1 & 0 & 0 & 0 \\
\hline & 0 & 0 & 0 & 0 & 0 & 0 & 0 & 0 & 0 & 0 & 0 & 0 & 1 \\
\hline & 0 & 0 & 0 & 0 & 0 & 0 & 0 & 0 & 0 & 0 & 0 & 0 & 0 \\
\hline & 0 & 0 & 0 & 0 & 0 & 0 & 0 & 0 & 0 & 0 & 0 & 0 & 0 \\
\hline \multirow[t]{6}{*}{$1.5 \mathrm{Y}$} & 7 & 7 & 5 & 4 & 2 & 1 & 0 & -2 & -3 & -5 & -9 & -13 & -15 \\
\hline & 0 & -3 & -4 & -3 & -2 & 0 & 0 & 1 & 1 & 3 & 3 & 1 & -4 \\
\hline & 0 & 0 & 1 & 1 & 1 & 0 & 0 & -1 & -1 & -1 & 0 & 0 & 0 \\
\hline & 1 & 0 & 0 & 0 & 0 & 0 & 0 & 0 & 0 & 0 & 0 & 0 & 1 \\
\hline & 0 & 0 & 0 & 0 & 0 & 0 & 0 & 0 & 0 & 0 & 0 & 0 & 0 \\
\hline & 0 & 0 & 0 & 0 & 0 & 0 & 0 & 0 & 0 & 0 & 0 & 0 & 0 \\
\hline \multirow[t]{6}{*}{$2 \mathrm{Y}$} & 9 & 9 & 7 & 5 & 3 & 2 & 0 & -2 & -4 & -7 & -12 & -18 & $\overline{-20}$ \\
\hline & 0 & -4 & -6 & -4 & -2 & -1 & 0 & 1 & 2 & 4 & 5 & 0 & -6 \\
\hline & 0 & 0 & 1 & 1 & 1 & 1 & 0 & -1 & -1 & -1 & -1 & 0 & 0 \\
\hline & 1 & 0 & 0 & 0 & 0 & 0 & 0 & 0 & 0 & 0 & 0 & 1 & 2 \\
\hline & 1 & 0 & 0 & 0 & 0 & 0 & 0 & 0 & 0 & 0 & 0 & 1 & 0 \\
\hline & 0 & 0 & 0 & 0 & 0 & 0 & 0 & 0 & 0 & 0 & 0 & 0 & 0 \\
\hline \multirow[t]{6}{*}{$\overline{3 Y}$} & 12 & 12 & 10 & 6 & 4 & 2 & 0 & -3 & -6 & -11 & -19 & -28 & -32 \\
\hline & 0 & -8 & -8 & -6 & -3 & -1 & 0 & 1 & 3 & 6 & 6 & -1 & -9 \\
\hline & 0 & 0 & 1 & 1 & 1 & 1 & 0 & -1 & -2 & -2 & -1 & 0 & 1 \\
\hline & 1 & 0 & 0 & 0 & 0 & 0 & 0 & 0 & 0 & 0 & 1 & 1 & 4 \\
\hline & 1 & 1 & 0 & 0 & 0 & 0 & 0 & 0 & 0 & 0 & 0 & 1 & 0 \\
\hline & 0 & 0 & 0 & 0 & 0 & 0 & 0 & 0 & 0 & 0 & 0 & 0 & 0 \\
\hline \multirow[t]{6}{*}{$\overline{5 Y}$} & 16 & 17 & 13 & 10 & 7 & 4 & 0 & -5 & -10 & -18 & -34 & -51 & -61 \\
\hline & -3 & -13 & -14 & -10 & -5 & -2 & 0 & 1 & 5 & 10 & 9 & -4 & -15 \\
\hline & 0 & 1 & 2 & 2 & 2 & 1 & 0 & -2 & -3 & -3 & -1 & 0 & 1 \\
\hline & 3 & 1 & 1 & 0 & 0 & 0 & 0 & 0 & 0 & 1 & 1 & 3 & 10 \\
\hline & 3 & 1 & 0 & 0 & 0 & 0 & 0 & 0 & 0 & 0 & 1 & 2 & -1 \\
\hline & 0 & 0 & 0 & 0 & 0 & 0 & 0 & 0 & 0 & 0 & 0 & 0 & 0 \\
\hline \multirow[t]{6}{*}{$\overline{10 Y}$} & 24 & 26 & 21 & 16 & 12 & 7 & -1 & -8 & -19 & -37 & -76 & -118 & $\overline{-14}$ \\
\hline & -16 & -26 & -26 & -17 & -9 & -4 & -1 & 2 & 8 & 20 & 13 & -17 & -23 \\
\hline & -1 & 1 & 3 & 4 & 4 & 2 & -1 & -3 & -5 & -6 & -2 & 1 & 2 \\
\hline & 4 & 2 & 1 & 1 & 1 & 1 & 1 & 1 & 1 & 2 & 3 & 8 & 36 \\
\hline & 6 & 2 & 0 & 0 & 0 & 1 & 1 & 1 & 0 & 0 & 3 & 5 & -6 \\
\hline & 0 & 0 & 0 & 0 & 0 & 1 & 1 & 1 & 1 & 0 & 0 & -1 & -1 \\
\hline
\end{tabular}


Table 18. CEV model $(\beta=0.8, \nu=0.25)$ : average per categories of strikes of errors in bps on the deltas using the 2 approximations AppDeltaLN $\left(1, x_{\mathrm{av}}\right)$ and AppDeltaLN $\left(2, x_{\mathrm{av}}\right)$.

\begin{tabular}{lrrrrr}
\hline & far ITM & ITM & \multicolumn{1}{c}{ ATM } & OTM & far OTM \\
\hline $3 \mathrm{M}$ & $-3.1 \mathrm{E}-2$ & $7.1 \mathrm{E}-2$ & $-1.9 \mathrm{E}-3$ & $-8.0 \mathrm{E}-2$ & $4.0 \mathrm{E}-2$ \\
& $-1.8 \mathrm{E}-3$ & $2.4 \mathrm{E}-4$ & $3.6 \mathrm{E}-3$ & $4.7 \mathrm{E}-4$ & $-2.4 \mathrm{E}-3$ \\
\hline $6 \mathrm{M}$ & $-1.1 \mathrm{E}-2$ & $2.3 \mathrm{E}-1$ & $-6.0 \mathrm{E}-3$ & $-2.3 \mathrm{E}-1$ & $4.8 \mathrm{E}-2$ \\
& $-4.6 \mathrm{E}-3$ & $5.0 \mathrm{E}-3$ & $1.1 \mathrm{E}-2$ & $4.4 \mathrm{E}-3$ & $-6.1 \mathrm{E}-3$ \\
\hline $1 \mathrm{Y}$ & $-4.0 \mathrm{E}-2$ & $4.5 \mathrm{E}-1$ & $-1.9 \mathrm{E}-2$ & $-4.5 \mathrm{E}-1$ & $7.9 \mathrm{E}-2$ \\
& $-1.4 \mathrm{E}-2$ & $1.7 \mathrm{E}-2$ & $3.0 \mathrm{E}-2$ & $1.2 \mathrm{E}-2$ & $-1.6 \mathrm{E}-2$ \\
\hline $1.5 \mathrm{Y}$ & $-1.9 \mathrm{E}-2$ & $6.8 \mathrm{E}-1$ & $-1.2 \mathrm{E}-1$ & $-6.6 \mathrm{E}-1$ & $1.4 \mathrm{E}-1$ \\
& $-2.4 \mathrm{E}-2$ & $2.8 \mathrm{E}-2$ & $5.3 \mathrm{E}-2$ & $2.4 \mathrm{E}-2$ & $-3.2 \mathrm{E}-2$ \\
\hline $2 \mathrm{Y}$ & $-2.4 \mathrm{E}-2$ & $8.7 \mathrm{E}-1$ & $-4.8 \mathrm{E}-2$ & $-9.6 \mathrm{E}-1$ & $2.1 \mathrm{E}-1$ \\
& $-3.6 \mathrm{E}-2$ & $4.4 \mathrm{E}-2$ & $7.9 \mathrm{E}-2$ & $3.9 \mathrm{E}-2$ & $-4.9 \mathrm{E}-2$ \\
\hline $3 \mathrm{Y}$ & $3.9 \mathrm{E}-2$ & $1.2 \mathrm{E}+0$ & $-9.4 \mathrm{E}-2$ & $-1.4 \mathrm{E}+0$ & $3.6 \mathrm{E}-1$ \\
& $-5.7 \mathrm{E}-2$ & $6.7 \mathrm{E}-2$ & $1.4 \mathrm{E}-1$ & $6.2 \mathrm{E}-2$ & $-9.5 \mathrm{E}-2$ \\
\hline $5 \mathrm{Y}$ & $6.3 \mathrm{E}-2$ & $2.0 \mathrm{E}+0$ & $-1.9 \mathrm{E}-1$ & $-2.3 \mathrm{E}+0$ & $6.5 \mathrm{E}-1$ \\
& $-1.2 \mathrm{E}-1$ & $1.4 \mathrm{E}-1$ & $2.8 \mathrm{E}-1$ & $1.2 \mathrm{E}-1$ & $-2.1 \mathrm{E}-1$ \\
\hline $10 \mathrm{Y}$ & $8.9 \mathrm{E}-2$ & $3.4 \mathrm{E}+0$ & $-5.6 \mathrm{E}-1$ & $-4.3 \mathrm{E}+0$ & $1.5 \mathrm{E}+0$ \\
& $-3.4 \mathrm{E}-1$ & $2.5 \mathrm{E}-1$ & $6.2 \mathrm{E}-1$ & $2.3 \mathrm{E}-1$ & $-6.1 \mathrm{E}-1$ \\
\hline
\end{tabular}


Table 19. CEV model $(\beta=0.2, \nu=0.25)$ : errors in bps on the deltas using the 6 approximations AppDeltaLN $\left(1, x_{0}\right), \operatorname{AppDeltaLN}(1, \mathrm{k}), \operatorname{AppDeltaLN}\left(1, x_{\mathrm{av}}\right), \operatorname{AppDeltaLN}\left(2, x_{0}\right)$, $\operatorname{AppDeltaLN}(2, \mathrm{k})$ and $\operatorname{AppDeltaLN}\left(2, x_{\mathrm{av}}\right)$.

\begin{tabular}{|c|c|c|c|c|c|c|c|c|c|c|c|c|c|}
\hline \multirow[t]{6}{*}{$3 \mathrm{M}$} & 19 & 27 & 25 & 18 & 11 & 6 & 0 & -6 & -11 & -17 & -30 & -31 & -27 \\
\hline & 9 & -1 & -10 & -13 & -9 & -3 & 0 & 3 & 7 & 9 & -3 & -7 & -6 \\
\hline & -1 & -1 & 0 & 2 & 2 & 2 & 0 & -2 & -2 & -1 & 0 & 0 & 0 \\
\hline & 5 & 3 & 2 & 1 & 1 & 0 & 0 & 0 & 0 & 1 & 2 & 3 & 5 \\
\hline & 3 & 3 & 2 & 0 & 0 & 0 & 0 & 0 & 0 & 0 & 1 & 0 & -1 \\
\hline & 0 & 0 & 0 & 0 & 0 & 0 & 0 & 0 & 0 & 0 & 0 & 0 & 0 \\
\hline \multirow[t]{6}{*}{$\overline{6 \mathrm{M}}$} & 51 & 45 & 34 & 24 & 16 & 9 & 0 & -8 & -15 & -31 & -41 & -59 & -58 \\
\hline & -7 & -29 & -27 & -19 & -10 & -3 & 0 & 4 & 9 & 17 & 13 & -6 & -11 \\
\hline & -2 & 2 & 4 & 5 & 5 & 3 & 0 & -2 & -4 & -3 & -2 & 0 & 1 \\
\hline & 10 & 3 & 2 & 2 & 1 & 1 & 1 & 1 & 1 & 2 & 2 & 5 & 13 \\
\hline & 10 & 3 & 1 & 1 & 1 & 1 & 1 & 1 & 0 & 1 & 2 & 3 & -2 \\
\hline & -1 & 0 & 0 & 0 & 1 & 1 & 1 & 1 & 1 & 0 & 0 & 0 & 0 \\
\hline \multirow[t]{6}{*}{$1 \mathrm{Y}$} & 99 & 90 & 59 & 46 & 24 & 13 & 1 & -10 & -32 & -53 & -93 & -117 & -113 \\
\hline & -54 & -69 & -52 & -37 & -11 & -3 & 1 & 5 & 19 & 32 & 12 & -14 & -9 \\
\hline & -3 & 3 & 9 & 11 & 8 & 5 & 1 & -3 & -7 & -7 & -2 & 1 & 1 \\
\hline & 24 & 10 & 5 & 4 & 3 & 2 & 2 & 2 & 3 & 4 & 7 & 12 & 41 \\
\hline & 28 & 9 & 2 & 2 & 2 & 2 & 2 & 2 & 1 & 1 & 6 & 6 & -4 \\
\hline & -2 & 0 & 1 & 1 & 2 & 2 & 2 & 2 & 1 & 1 & 0 & -1 & 0 \\
\hline \multirow{6}{*}{$1.5 \mathrm{Y}$} & 146 & 126 & 86 & 70 & 43 & 16 & 2 & -26 & -38 & -77 & -140 & -183 & -180 \\
\hline & -123 & -114 & -81 & -60 & -23 & -3 & 2 & 13 & 21 & 45 & 12 & -27 & -10 \\
\hline & -2 & 7 & 15 & 17 & 15 & 7 & 2 & -7 & -9 & -10 & -3 & 1 & 1 \\
\hline & 35 & 16 & 10 & 8 & 5 & 4 & 4 & 4 & 4 & 7 & 13 & 24 & 72 \\
\hline & 37 & 12 & 3 & 3 & 4 & 4 & 4 & 3 & 2 & 2 & 11 & 7 & -6 \\
\hline & -3 & 0 & 2 & 3 & 4 & 4 & 4 & 3 & 3 & 1 & -1 & -1 & -1 \\
\hline \multirow[t]{6}{*}{$\overline{2 \mathrm{Y}}$} & 189 & 163 & 17 & 81 & 51 & 36 & 3 & -29 & -58 & -102 & -173 & -254 & -224 \\
\hline & -197 & -164 & -115 & -63 & -23 & -11 & 3 & 14 & 33 & 58 & 23 & -40 & -4 \\
\hline & 0 & 11 & 20 & 23 & 19 & 14 & 3 & -7 & -13 & -13 & -5 & 2 & 1 \\
\hline & 50 & 24 & 15 & 11 & 7 & 6 & 5 & 6 & 7 & 11 & 18 & 40 & 125 \\
\hline & 45 & 15 & 5 & 5 & 6 & 6 & 5 & & 3 & 3 & 14 & 4 & -3 \\
\hline & -4 & 0 & 3 & 5 & 6 & 6 & 5 & 5 & 4 & 2 & -1 & -2 & -1 \\
\hline \multirow[t]{6}{*}{$3 \mathrm{Y}$} & 262 & 218 & 188 & 118 & 82 & 45 & 6 & -33 & -86 & -157 & -286 & -393 & -354 \\
\hline & -377 & -245 & -204 & -93 & -40 & -10 & 6 & 18 & 49 & 82 & -3 & -54 & -3 \\
\hline & 0 & 24 & 30 & 36 & 31 & 20 & 6 & -7 & -19 & -18 & -4 & 3 & 1 \\
\hline & 95 & 36 & 29 & 19 & 14 & 11 & 10 & 10 & 13 & 0 & 35 & 77 & 230 \\
\hline & 51 & 13 & 9 & 9 & 11 & 11 & 10 & 8 & 5 & 6 & 26 & -4 & -2 \\
\hline & -8 & 2 & 5 & 9 & 10 & 10 & 10 & 9 & 6 & 3 & -2 & -4 & -1 \\
\hline \multirow[t]{6}{*}{$\overline{5 Y}$} & 373 & 323 & 253 & 197 & 131 & 85 & 11 & -61 & -148 & -258 & -497 & -684 & -592 \\
\hline & -641 & -409 & -276 & -169 & -60 & -19 & 11 & 34 & 84 & 121 & -43 & -59 & 0 \\
\hline & 0 & 43 & 59 & 64 & 53 & 38 & 11 & -13 & -30 & -28 & -3 & 4 & 0 \\
\hline & 156 & 62 & 47 & 39 & 28 & 23 & 20 & 20 & 26 & 38 & 73 & 170 & 533 \\
\hline & -156 & -19 & 3 & 15 & 22 & 23 & 20 & 15 & 8 & 13 & 38 & -22 & 0 \\
\hline & -27 & 2 & 13 & 19 & 22 & 21 & 20 & 17 & 12 & 5 & -6 & -6 & 0 \\
\hline \multirow[t]{6}{*}{$\overline{10 Y}$} & 390 & 381 & 325 & 262 & 06 & 134 & 18 & -102 & -269 & -529 & -1075 & $5-1477$ & $7-11$ \\
\hline & -174 & -486 & -447 & -269 & -123 & -36 & 18 & 56 & 49 & 175 & -127 & -23 & 0 \\
\hline & -71 & -8 & 39 & 76 & 78 & 58 & 18 & -21 & -53 & -44 & 0 & 4 & 0 \\
\hline & 75 & -5 & 3 & 25 & 32 & 34 & 38 & 43 & 57 & 88 & 189 & 536 & 172 \\
\hline & -1252 & -598 & -237 & -43 & 18 & 7 & 38 & 32 & 15 & 47 & 5 & -21 & 0 \\
\hline & -157 & -97 & -49 & -3 & 23 & 34 & 38 & 36 & 26 & 6 & -16 & -10 & 0 \\
\hline
\end{tabular}

